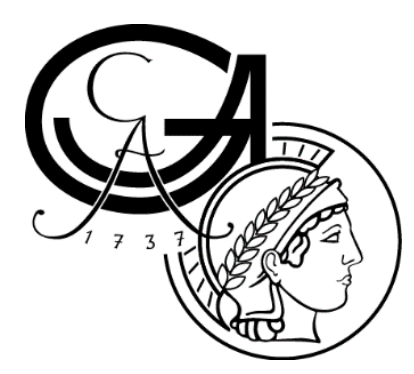

\title{
Gbp2 and Hrb1 continue their mRNA quality control in the cytoplasm and take part in Nonsense Mediated Decay
}

\author{
Dissertation \\ for the award of the degree \\ "Doctor rerum naturalium" \\ of the Georg-August-Universität Göttingen \\ within the doctoral program "Molecular Biology" \\ of the Georg-August University School of Science (GAUSS) \\ submitted by \\ Sebastian Grosse \\ from Minden \\ Göttingen 2019
}




\section{Thesis Committee}

Prof. Dr. Heike Krebber

Department of Molecular Genetics

Institute for Microbiology and Genetics

Prof. Dr. Reinhard Lührmann

Department of Cellular Biochemistry

Max Planck Institute for Biophysical Chemistry

Prof. Dr. Jörg Großhans

Department of Developmental Biochemistry

University Medical Center Göttingen

\section{Members of the Examination Board}

Referee: $\quad$ Prof. Dr. Heike Krebber

Department of Molecular Genetic

Institute for Microbiology and Genetics

$2^{\text {nd }}$ Referee: Prof. Dr. Reinhard Lührmann

Department of Cellular Biochemistry

Max Planck Institute for Biophysical Chemistry

$3^{\text {rd }}$ referee: Prof. Dr. Jörg Großhans

Department of Developmental Biochemistry

University Medical Center Göttingen

\section{Further members of the Examination Board}

Prof. Dr. Jörg Stülke

Department of General Microbiology

Institute for Microbiology and Genetics

Prof. Dr. Stefani Pöggeler

Department of Genetics of Eukaryotic Microorganisms

Institute for Microbiology and Genetics

Prof. Dr. Ralf Ficner

Institute for Microbiology and Genetics

Department of Molecular Structural Biology

Date of oral examination: 27.08.2019 


\section{Affidavit}

I hereby declare that I prepared this doctoral thesis titled "Gbp2 and Hrb1 continue their mRNA quality control in the cytoplasm and take part in Nonsense Mediated Decay" independently and with no other sources and aids than quoted.

Göttingen, June 2019

Sebastian Grosse 


\section{Table of contents}

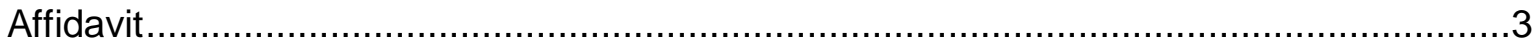

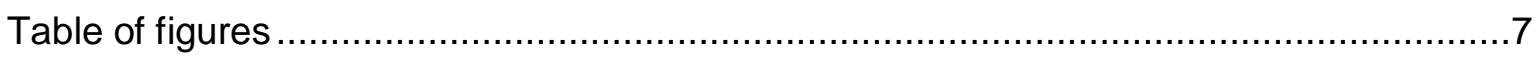

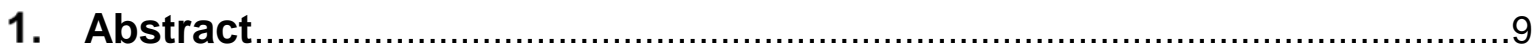

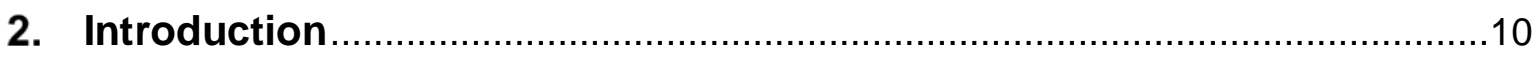

2.1. Nuclear mRNA processing and export...................................................... 10

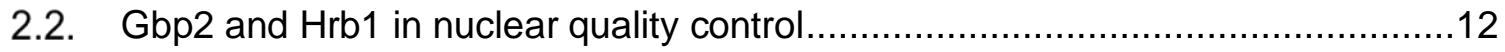

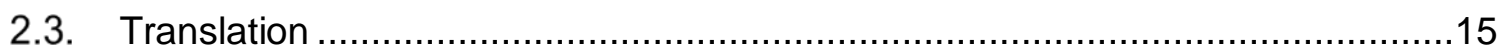

2.3.1. Translation initiation and elongation ..................................................... 15

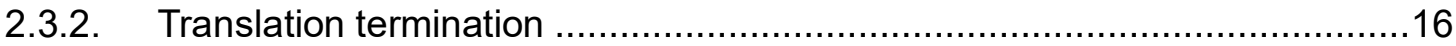

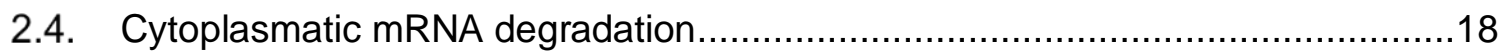

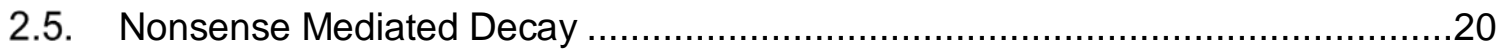

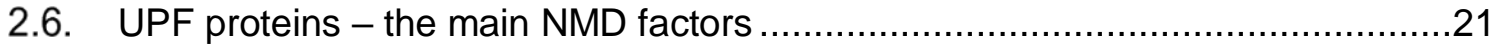

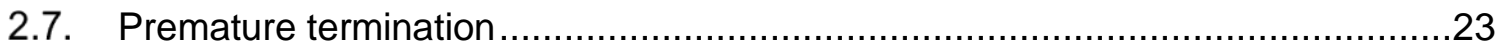

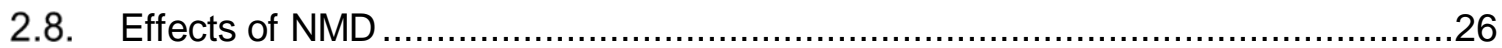

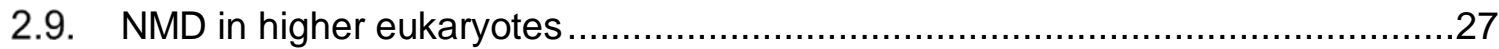

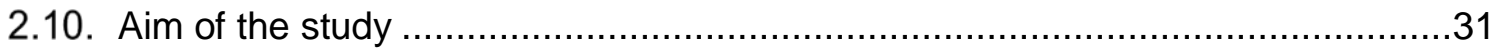

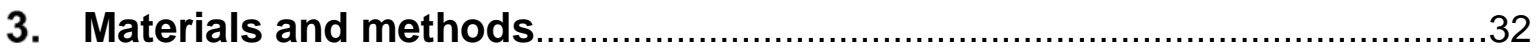

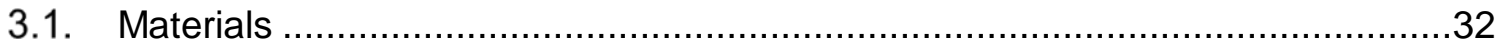

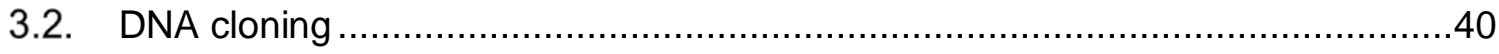

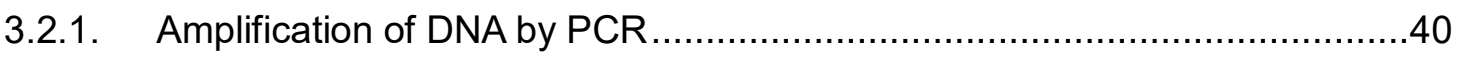

3.2.2. Cleavage of DNA by restriction digestion ..........................................41

3.2.3. Agarose gel electrophoresis ................................................................

3.2.4. DNA extraction from agarose gels and enzymatic reactions .....................42

3.2.5. Extraction of genomic DNA from S. cerevisiae cells ..............................42

3.2.6. Measurement of DNA and RNA concentrations ...................................43

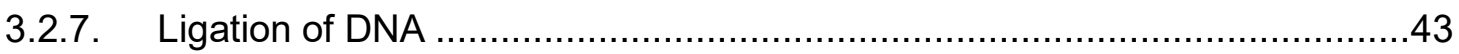

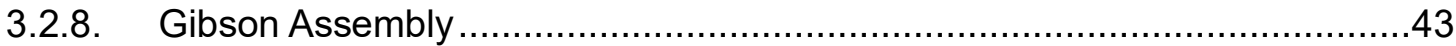

3.2.9. Transformation of E. coli with plasmid DNA …..........................................44 
3.2.10. Plasmid purification from E. coli cultures .........................................44

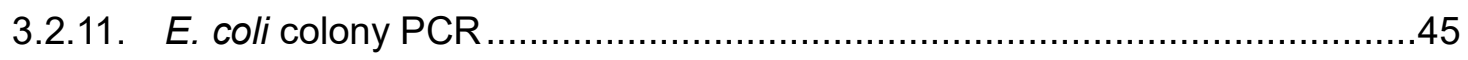

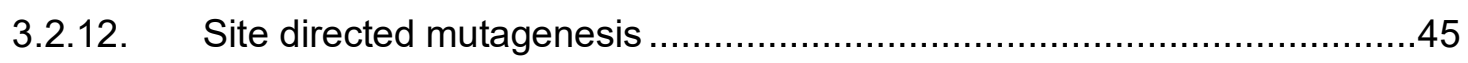

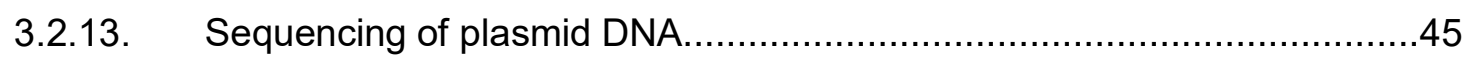

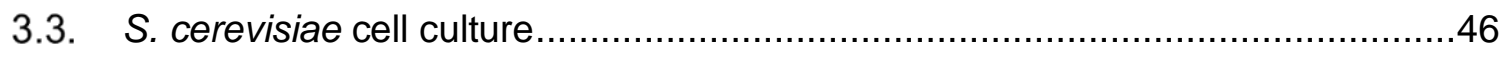

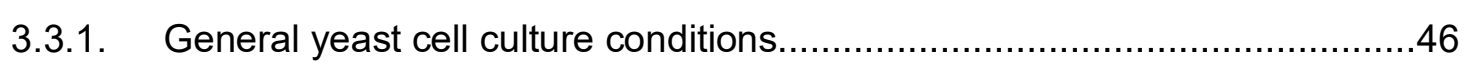

3.3.2. Measurement of yeast cell density in liquid cultures ..............................47

3.3.3. Transformation of yeast cells with plasmid DNA ................................47

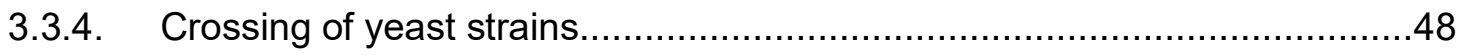

3.3.5. Induction of galactose responsive promoters ....................................49

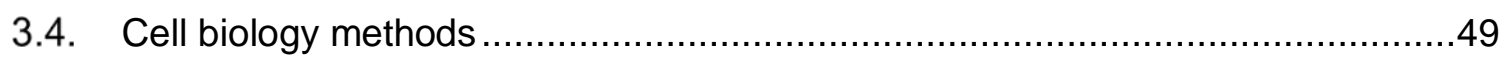

3.4.1. Growth analysis of yeast strains …............................................ 49

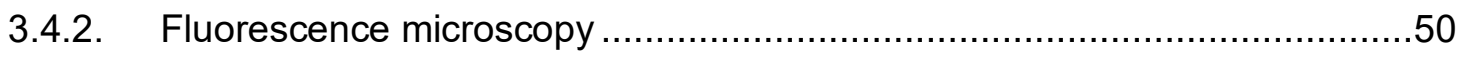

3.5. Biochemical methods - protein analysis................................................ 51

3.5.1. Immunoprecipitation of GFP tagged proteins …................................ 51

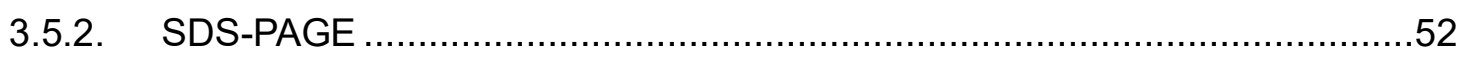

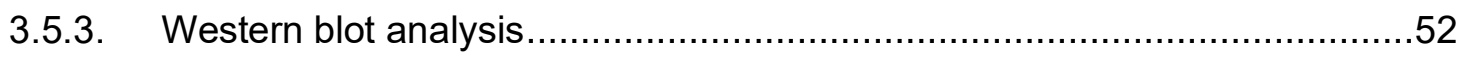

3.5.4. Yeast cell lysis for Western blot analysis ............................................ 53

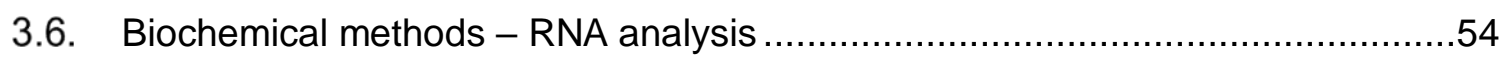

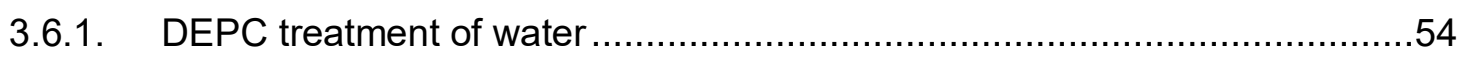

3.6.2. RNA Co-Immunoprecipitation with GFP-tagged proteins...........................54

3.6.3. RNA isolation from whole-cell lysates ...............................................56

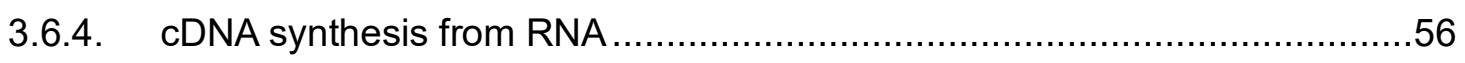

3.6.5. Quantitative Polymerase Chain Reaction (qPCR) ...............................56

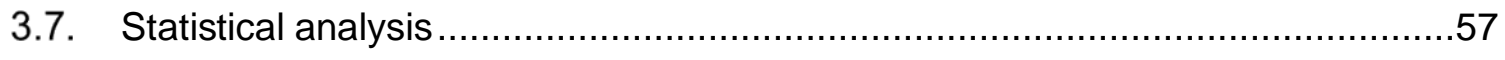

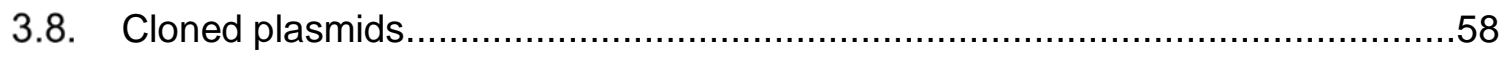

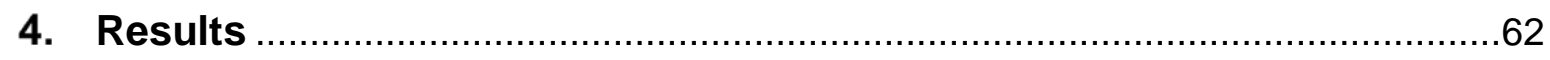

4.1. Gbp2 and Hrb1 are novel factors in cytoplasmic quality control.......................62

4.2. Upf 1 mediated degradation of NMD targets is defective in gbp2 $\Delta$ hrb1 $\Delta$ cells....63 
4.3. Gbp2 and Hrb1 are involved in translation inhibition of NMD targets

4.4. The role of Gbp2 and Hrb1 in translation inhibition is Upf1 and PTC dependent 68

4.5. Gbp2 and Hrb1 are not involved in NMD of PGK1 ${ }^{P T C}$ 69

4.6. Translation cannot terminate close to the start codon. 70

4.7. The RGG motif proteins Npl3, Sbp1 and Scd6 are not involved in the translation inhibition of $M Y C-C B P 80^{P T C}$. .71

4.8. elF4E binding to $C B P 80^{P T C}$ is not regulated by the Upf 1 pathway .....................72

4.9. Gbp2 and Hrb1 mis-localise to the cytoplasm through excessive NMD .............74

4.10. Gbp2 and Hrb1 interact with the cytoplasmic degradation machinery ................76

4.11. Hrb1 promotes the recruitment of Dcp1 to NMD targets .................................78

4.12. Gbp2 and Hrb1 are not required to recruit $\mathrm{Xrn} 1$ to the $C B P 80^{P T C} \mathrm{RNA} \ldots \ldots \ldots \ldots . . . . .80$

4.13. Gbp2 and Hrb1 interact with each other and themselves ...............................81

4.14. Gbp2 and Hrb1 have a stabilising effect on normal mRNAs ............................82

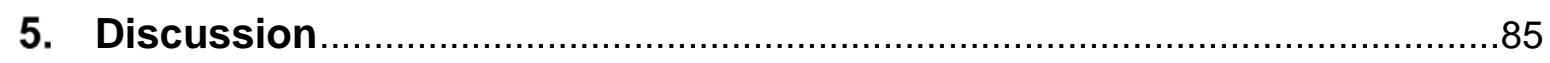

5.1. Gbp2 and Hrb1 are part of cytoplasmic quality control ................................85

5.2. Degradation of NMD targets is defective in gbp2 $\Delta$ hrb1 $\Delta$ cells ........................86

5.3. Gbp2 and Hrb1 are also involved in translation inhibition of NMD targets..........88

5.4. The binding of elF4E to $C B P 80^{P T C}$ is not regulated by the Upf1 pathway ...........93

5.5. Gbp2 and Hrb1 are directly involved in the NMD pathway ...............................95

5.6. Gbp2 and Hrb1 interact with the cytoplasmic degradation machinery ...............97

5.7. Hrb1 promotes the recruitment of the decapping enzyme to $M Y C-C B P 80^{P T C} \ldots . .98$

5.8. Gbp2 and Hrb1 promote the stability of normal mRNAs in the cytoplasm .........101

5.9. Gbp2 and Hrb1 may contribute to the mRNP structure ................................101

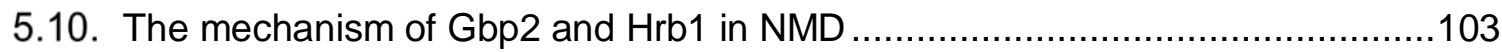

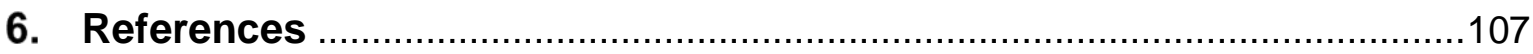

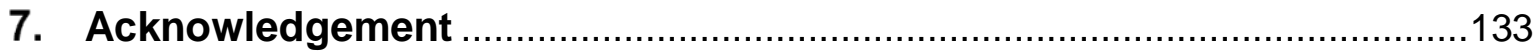




\section{Table of figures}

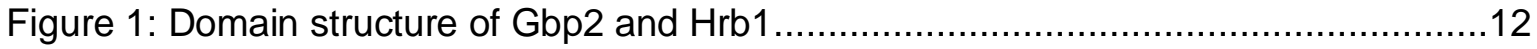

Figure 2: Nucleo-cytoplasmic shuttling of Gbp2 and Hrb1 ..........................................14

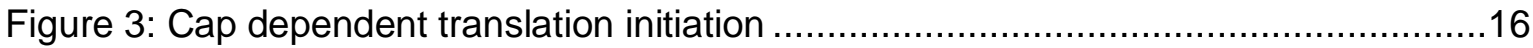

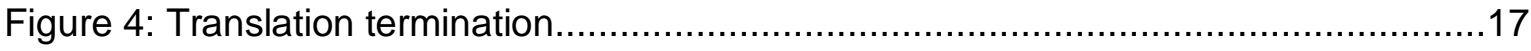

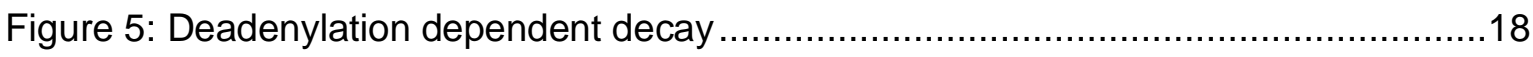

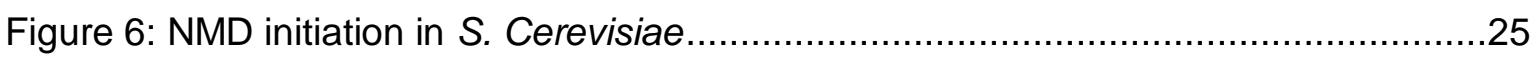

Figure 7: Hsp104-RFP aggregates in mutants of cytoplasmic quality control. ..................62

Figure 8: Gbp2 and Hrb1 are required for PTC induced destabilisation of CBP80 mRNA.

Figure 9: Gbp2 and Hrb1 are required for PTC induced destabilisation of DBP2 mRNA. .65 Figure 10: Gbp2 and Hrb1 are involved in the Upf1 mediated destabilisation of the NMD

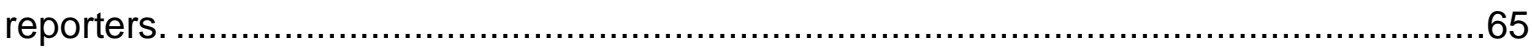

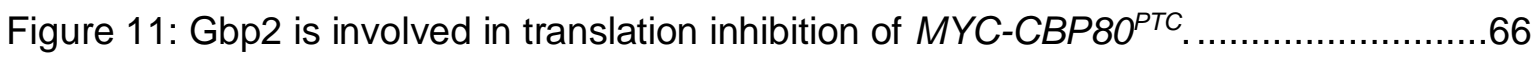

Figure 12: Gbp2 and Hrb1 are involved in translation inhibition of $M Y C-D B P 2^{P T C} \ldots \ldots \ldots . . . .67$

Figure 13: Translation inhibition of Gbp2 and Hrb1 is Upf1 dependent............................68

Figure 14: Gbp2 and Hrb1 do not inhibit translation of wild typical CBP80 and DBP2.....69

Figure 15: Gbp2 and Hrb1 show no influence on MYC-PGK1 ${ }^{P T C}$ translation.....................69

Figure 16: Translation cannot terminate at a PTC close to the 5' end. ……....................70

Figure 17: The RGG motif containing translation inhibitors Npl3, Scd6 and Sbp1 show no

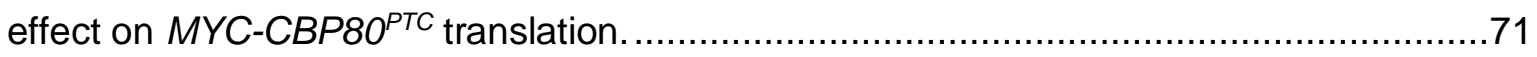

Figure 19: $C B P 80^{P T C}$ is not translated like wild typical $C B P 80$ in upf $1 \triangle$ cells. ..................73

Figure 18: The elF4E binding to $C B P 80^{P T C}$ is not increased in gbp2 $\triangle$ and upf1 $\triangle$ cells. ...73 Figure 20: Nuclear re-import of Gbp2 is disturbed by high expression of NMD reporters in the absence of Xrn1.

Figure 21: Nuclear re-import of Hrb1 is disturbed by high expression of NMD reporters in the absence of Xrn1. .75

Figure 22: Gbp2 and Hrb1 physically interact with the cytoplasmic degradation machinery.

Figure 23: The interaction between Dcp1 and Upf1 is decreased in gbp2 $\Delta$ rb1 $\Delta$ cells. ..78

Figure 24: Hrb1 promotes the binding of Dcp1 to the $C B P 80^{P T C}$ NMD reporter .79

Figure 25: $C B P 80^{P T C}$ NMD reporter binding to Xrn1-GFP is unaltered in gbp2 $\triangle$ hrb1 $\Delta$ cells.

Figure 26: Gbp2 and Hrb1 show RNase resistant interactions with each other and themselves.

Figure 27: Loss of Gbp2 and Hrb1 leads to reduced mRNA levels. 
Figure 28: The absence of Gbp2 and Hrb1 causes an Xrn1 dependent destabilisation of

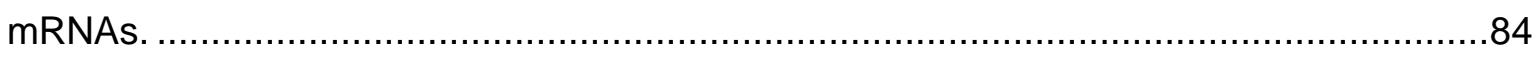

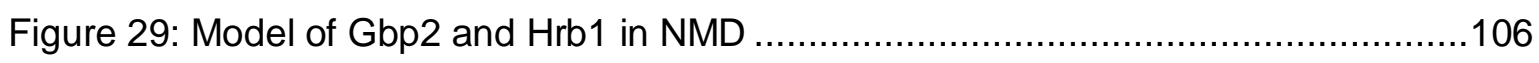




\section{Abstract}

The eukaryotic mRNA life cycle is orchestrated by many pathways. Every aspect of this life cycle is associated with quality control factors. This tight coupling of mRNA transcription, processing, export and translation with different parts of the quality control machinery ensures the production of correct polypeptides. Surveillance of mRNA maturation involves several different pathways. Nuclear quality control checks mRNA processing: capping, splicing and polyadenylation. In S. cerevisiae the nuclear guard proteins Npl3, Gbp2, Hrb1 and Nab2 promote mRNA export only when these processing steps have occurred correctly. An aberrant mRNA is not only retained in the nucleus, it is also targeted for rapid RNA decay. An mRNA that passes the nuclear quality control checks and reaches the cytoplasm is subject to three different cytoplasmic surveillance pathways, which monitor if an mRNA is translated correctly. The cytoplasmic quality control inhibits translation and degrades the mRNA and the newly synthesised polypeptide. One of these pathways is Nonsense Mediated Decay (NMD), which targets mRNAs that undergo premature translation termination.

The yeast SR-like proteins Gbp2 and Hrb1 are nuclear guard proteins that monitor splicing. They recruit the degradation machinery to remove mRNAs that fail to be spliced correctly. While it was known, that Gbp2 and Hrb1 accompany mRNA into the cytoplasm until translation, it remained unknown why they stay on the mRNA. Here we show that Gbp2 and Hrb1 continue their quality control in the cytoplasm and are part of the NMD pathway. Aggregation of Hsp104 shows that cytoplasmic quality control is defective without Gbp2 and Hrb1. In their absence both NMD induced mRNA decay and translation inhibition are impaired. Our data suggest that the proteins are directly involved in the NMD pathway. If the degradation is impaired, ongoing NMD stalls Gbp2 and Hrb1 in the cytoplasm. This agrees with other data showing physical interactions with the main NMD effectors Upf1, Upf2 and Upf3. We gathered evidence that Upf1 mediates the direct recruitment of degradation factors to NMD targets in vivo. Both Gbp2 and Hrb1 interact with the cytoplasmic degradation machinery. Strikingly, Upf1 requires Hrb1 for the correct recruitment of the decapping enzyme, while it requires Gbp2 for effective translation inhibition. Gbp2, as an RGG motif protein, has the potential to be a general translation inhibitor. However, our results suggest, that it is NMD specific. Further, Gbp2 and Hrb1, which preferentially associate with the 5' ends of mRNAs, might be structural elements of cytoplasmic mRNPs. In their absence, wild typical mRNAs are more susceptible to degradation by the 5'-3' exonuclease Xrn1 in vivo. The association of Gbp2 and Hrb1 with the cytoplasmic degradation machinery is a new facet in understanding the intricate machinery of mRNA quality control and might offers a new perspective in unravelling the functions of SR-proteins in eukaryotes. 


\section{Introduction}

\subsection{Nuclear mRNA processing and export}

The eukaryotic mRNA life cycle is orchestrated by a multitude of different factors in many different pathways. Already co-transcriptionally, the mRNA is processed by three different mechanisms -5 ' capping, intron splicing and 3' polyadenylation (Hocine et al., 2010). At the 5' end, the capping enzymes Cet1, Ceg1 and Abd1 form the 7-methylguanosine cap (Ghosh and Lima, 2010; Mao et al., 1995; Tsukamoto et al., 1997). The cap protects the mRNA from degradation. In the nucleus, the cap binding complex (CBC), consisting of Cbp20 and Cbp80, covers the cap. The CBC promotes spliceosome assembly (Lewis et al., 1996) and antagonises premature 3 ' end formation (Wong et al., 2007), but it might also promote degradation of mRNAs that are retained in the nucleus (Das et al., 2000). The CBC shuttles on the mRNA into the cytoplasm, where it can also facilitate translation initiation, likely in early translation (Fortes et al., 2000).

Intron containing mRNAs undergo splicing (reviewed in Will and Lührmann, 2011). The intron sequences are removed by transesterification at the splice sites and the branch point. The snRNPs U1, U2, U4/U6 and U5 assemble on an intron, thereby forming the spliceosome to facilitate the splicing reaction. The spliceosome assembly, rearrangement and recycling is promoted by ATP/GTP dependent helicases.

The 3' end is formed by cleavage of the mRNA followed by polyadenylation. For this processing step, the cleavage and polyadenylation factors assemble at polyadenylation signals in the mRNA sequence (Zhao et al., 1999). The poly(A) tail promotes mRNA stability and translation. It is the binding platform for Nab2 and Pab1, which are important for the correct poly(A) tail length and mRNA export. During the mRNA export, Nab2 and Pab1 shuttle into the cytoplasm (Brune et al., 2005; Hector, 2002; Meinel and Sträßer, 2015).

Nab2 belongs to a group of proteins - together with the proteins Gbp2, Hrb1 and Npl3 that are adapters for the export receptor Mex67 to regulate mRNA export (Gilbert and Guthrie, 2004; Häcker and Krebber, 2004; Hackmann et al., 2014; Lee et al., 1996; Stutz et al., 2000; Windgassen and Krebber, 2003; Zander et al., 2016). These factors shuttle into the cytoplasm with the mRNA. Mex67 forms a heterodimer with Mtr2 and is essential for mRNA export. The Mex67-Mtr2 heterodimer can interact with mRNA and the nuclear pore complex (NPC) (Santos-Rosa et al., 1998; Segref et al., 1997). Phenylalanine glycine (FG) rich extensions presumably form a meshwork through hydrophobic interaction in the inner channel of the nuclear pore. This meshwork likely blocks the entry of larger compounds into the NPC (reviewed in Strambio-De-Castillia et al., 2010). Mex67-Mtr2 can interact with the

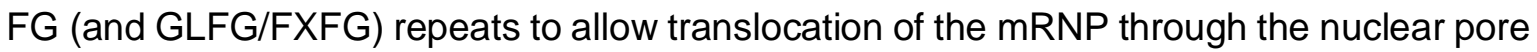
(Strasser et al., 2000). On the cytoplasmic side of the NPC, the DEAD box helicase Dbp5 
removes Mex67. This requires ATP, and the ATPase stimulating cofactors Gle1 and inositol hexakisphosphate $\left(\mathrm{IP}_{6}\right)$. Through the removal of Mex67 on the cytoplasmic side of the NPC, an mRNA, which is in the translocation process, cannot move backwards. This step imposes directionality on the mRNA export (Tieg and Krebber, 2013). Dbp5 also promotes the dissociation of Nab2, it is unknown how it specifically removes some proteins and leaves factors that are relevant for translation on the mRNA (Ledoux and Guthrie, 2011).

Presumably, the mRNP needs to be sufficiently covered with Mex67-Mtr2 to be able to move through the NPC (Hackmann et al., 2014; Zander et al., 2016). This coverage of an mRNP with Mex67 appears to be controlled by the Mex67 adapter proteins Nab2, Npl3, Hrb1 and Gbp2. The adapters interact with Mex67 and are likely able to recruit it to the mRNP to promote export. However, Mex67 can bind to RNA directly and the adapters are rather guard proteins, that prevent mRNA export if necessary. They are part of the nuclear quality control (or surveillance) machinery, which prevents the nuclear export of incorrectly processed mRNAs. Likely, each processing step is coupled to Mex67 binding via a specific guard protein. Nab2 controls 3' end formation (Hector, 2002), Gbp2 and Hrb1 control splicing (see 2.2) and Npl3 controls 5' capping (unpublished data, laboratory of Heike Krebber). A model was proposed, in which all of these processing steps have to occur correctly to allow Mex67 binding by the various guard proteins, to acquire export competence (Hackmann et al., 2014; Zander et al., 2016). Interestingly, heat shock responsive mRNAs circumvent the guard proteins and bind Mex67 directly, likely to ensure a fast mRNA export for a timely heat shock response (Zander et al., 2016). The nuclear basket is also involved in exporting or retaining mRNAs. The most important quality control factor of the nuclear basket is likely Mlp1. It is bound to the nuclear pore through Nup60 and it can interact with Mex67. Probably it can direct the export competent mRNAs towards the nuclear pore. However, Mlp1 also interacts with the quality control machinery and can prevent export of aberrant transcripts (Galy et al., 2004; Green et al., 2003; Hackmann et al., 2014).

The quality control machinery does not only prevent the export of faulty transcripts, it also induces mRNA decay via Rat1, a nuclear 5'-3' exonuclease, or the exosome, a protein complex with 3'-5' exonuclease activity (Tutucci and Stutz, 2011). A kinetic quality control model was also proposed, which suggests that mRNA export and degradation are competing. Correct mRNA processing might allow the efficient mRNP remodelling steps required for export. If export is efficient, the degradation machinery might not be able to assemble on the mRNA. Inefficient mRNA export, on the other hand, may allow the degradation machinery to take over (Tutucci and Stutz, 2011). 


\subsection{Gbp2 and Hrb1 in nuclear quality control}

Gbp2 and Hrb1 contain three RNA recognition motif (RRM) domains and a serine arginine rich (SR) domain (see Figure 1). The SR domains of Gbp2 has 9 SR/RS motifs as well as 4 RGG motifs. Hrb1's SR domain includes 10 SR/RS motifs and 2 RGG motifs (Häcker and Krebber, 2004; Windgassen and Krebber, 2003). They both belong to the group of SR-like proteins. In contrast to canonical SR-proteins, their SR domain is N-terminal (instead of Cterminal) and they possess a unique C-terminal pseudo-RRM (RRM3), which is dispensable for RNA binding but facilitates protein-protein interactions (Graveley and Hertel, 2005; Häcker and Krebber, 2004; Martínez-Lumbreras et al., 2016; Windgassen and Krebber, 2003). Like all SR proteins, they bind RNA via their regular RRMs. It was described that their RRM1 and RRM2 both contribute to RNA binding. Likely the two RRMs form a binding platform together (Martínez-Lumbreras et al., 2016).

\section{$\mathrm{N}-\mathrm{SR} / \mathrm{RGG}-\mathrm{RRM} 1-\mathrm{RRM} 2-\mathrm{RRM} 3-\mathrm{C}$}

\section{Figure 1: Domain structure of Gbp2 and Hrb1}

The SR-like proteins contain one SR/RGG domain, two RRMs and one pseudo RRM. (Adapted from Häcker and Krebber, 2004)

Yeast has a third SR-like protein: Npl3. Npl3 has a C-terminal SR-domain, which also contains RGG motifs, and two RNA recognition motifs (Häcker and Krebber, 2004). Gbp2 and Hrb1 share $47 \%$ amino acid sequence identity with each other and $27 \%$ and $23 \%$, respectively, with Npl3 (Häcker and Krebber, 2004). All three are mostly nuclear, but they shuttle into the cytoplasm during mRNA export. Their nuclear re-import is promoted by the yeast SR protein kinase Sky1 and they are guided through the NPC via the karyopherin Mtr10 (Häcker and Krebber, 2004; Windgassen and Krebber, 2003; Windgassen et al., 2004). In the absence of Sky1, the nuclear re-import can be rescued by high levels of Mtr10. Thus, the phosphorylation might increase the binding affinity to Mtr10. However, while the Npl3 dissociation from polysomes is Mtr10 dependent, it is not influenced by Sky1. Similarly, Sky1 showed no effect on the polysome dissociation of Gbp2 either. (Windgassen et al., 2004). The concrete mechanism of dissociation and nuclear re-import is still unclear. For Npl3 it is known that it is dephosphorylated by Glc7 and methylated by Hmt1 in the nucleus (Gilbert and Guthrie, 2004; Siebel and Guthrie, 1996; Wong et al., 2010). Gbp2 and Hrb1 are very likely also imported with a phosphorylated SR domain. Gbp2 is likely phosphorylated by Sky1 at S13, S15 an S17 and seems to require at least S15 and S17 phosphorylation for efficient nuclear import (Windgassen and Krebber, 2003). Presumably, Gbp2 and Hrb1 are also dephosphorylated in the nucleus. The RGG region of Gbp2 is also methylated, probably by Hmt1, which is known to methylate RGG motifs (Erce et al., 2013; 
Lien et al., 2016; Wong et al., 2010; Xu et al., 2003). The three SR like proteins have no known direct orthologue in higher eukaryotes. Humans have 12 different SR proteins and several SR-like proteins. The SR-proteins SRSF1, SRSF3 and SRSF7 shuttle into the cytoplasm and were implicated in mRNA export (Huang and Steitz, 2005). The main role of SR and SR-like proteins in higher eukaryotes appears to be the regulation of constitutive and alternative splicing (Long and Caceres, 2009).

In the nucleus, Npl3 is involved in mRNA-export and quality-control (Lee et al., 1996; Zander et al., 2016), rRNA processing (Russell and Tollervey, 1992), correct transcription termination (Bucheli and Buratowski, 2005; Dermody et al., 2008) and presumably telomere maintenance (Lee-Soety et al., 2012). It is apparently not a bona-fide splicing factor but loss of Npl3 causes defects in the spliceosome assembly (Kress et al., 2008). Npl3 is presumably recruited to the mRNA by the RNA polymerase II very early (Lei, 2001). Possibly, loss of Npl3 causes problems with early mRNA processing, which might indirectly lead to defective downstream events (unpublished data, laboratory of Heike Krebber).

Gbp2 and Hrb1 are not actual splicing factors either. However, they were shown to interact with the late splicing factors Prp17 and Prp43, which are involved in the second catalytic step and spliceosome recycling, respectively (Hackmann et al., 2014). Gbp2 and Hrb1 act as nuclear retention factors for unspliced pre-mRNA. As described in section 2.1, they can interact with Mex67 and may be able to promote mRNA export for spliced mRNAs. However, their main function appears to be preventing the export of unsuccessfully spliced mRNAs from the nucleus. It was observed, that intron containing mRNAs leak into the cytoplasm in the absence of Gbp2 or Hrb1. Thus, when their splicing associated quality control step is missing, the remaining export machinery can export aberrant (pre-)mRNAs. In this pathway, Gbp2 and Hrb1 likely cooperate with the nuclear basket protein Mlp1, with which they physically interact. Loss of Mlp1 also causes leakage of intron containing premRNA into the cytoplasm. While Gbp2 and Hrb1 are dispensable for cell growth under normal conditions, they show genetic interactions with Mlp1, and gbp2 $h r b 1 \Delta$ causes synthetic lethality with mutants of the splicing factors Prp8, Prp17 and Prp43. Further, Gbp2 and Hrb1 do not only prevent export, but seem to actively induce decay during their surveillance function. They physically link the 3'-5' degradation machinery to the spliceosome. Both Gbp2 and Hrb1 show RNase resistant interaction with the helicase Mtr4 (Hackmann et al., 2014). Mtr4 is part of the TRAMP (Trf4/Air2/Mtr4 Polyadenylation) complex, which is an essential cofactor of the nuclear exosome (Callahan and Butler, 2010; Jia et al., 2011). The exosome is a large multi-subunit complex that degrades mRNAs in 3'5' direction, for which it requires regulatory cofactors. While the nuclear cofactor is the TRAMP complex, the cytoplasmic exosome cooperates with the Ski complex (see 2.4; Dunn et al., 2005; Libri et al., 2002). The nuclear exosome contains two exonucleolytic subunits, 
Rrp6 and Rrp44(Dis3). The cytoplasmic exosome contains only Rrp44 (reviewed in Sloan et al., 2012). The TRAMP complex and the exosome also have regulatory functions, such as degradation of intron sequences (Kong et al., 2014; San Paolo et al., 2009), and processing of rRNA and other noncoding RNAs (Allmang et al., 1999).

The interaction of Mex67 and Mtr4 with Gbp2-Hrb1 is mutually exclusive in vivo. It was proposed that Gbp2 and Hrb1 might interact with the TRAMP complex by default and correct splicing may trigger an interaction shift to allow mRNA export (Hackmann et al., 2014).

While Gbp2 and Hrb1 have a preferential binding for transcripts from intron containing genes, they appear to associate with all mRNAs (Hackmann et al., 2014). They interact with the THO complex, which may be relevant for their recruitment to the mRNA (Häcker and Krebber, 2004; Hurt et al., 2004). The THO complex consists of Hrp1, Tho2, Thp2, Mft1 and Tex1 (Chávez et al., 2000; Peña et al., 2012). It promotes transcription elongation and is also involved in mRNA export. Together with the DEAD box helicase Sub2 and the Mex67

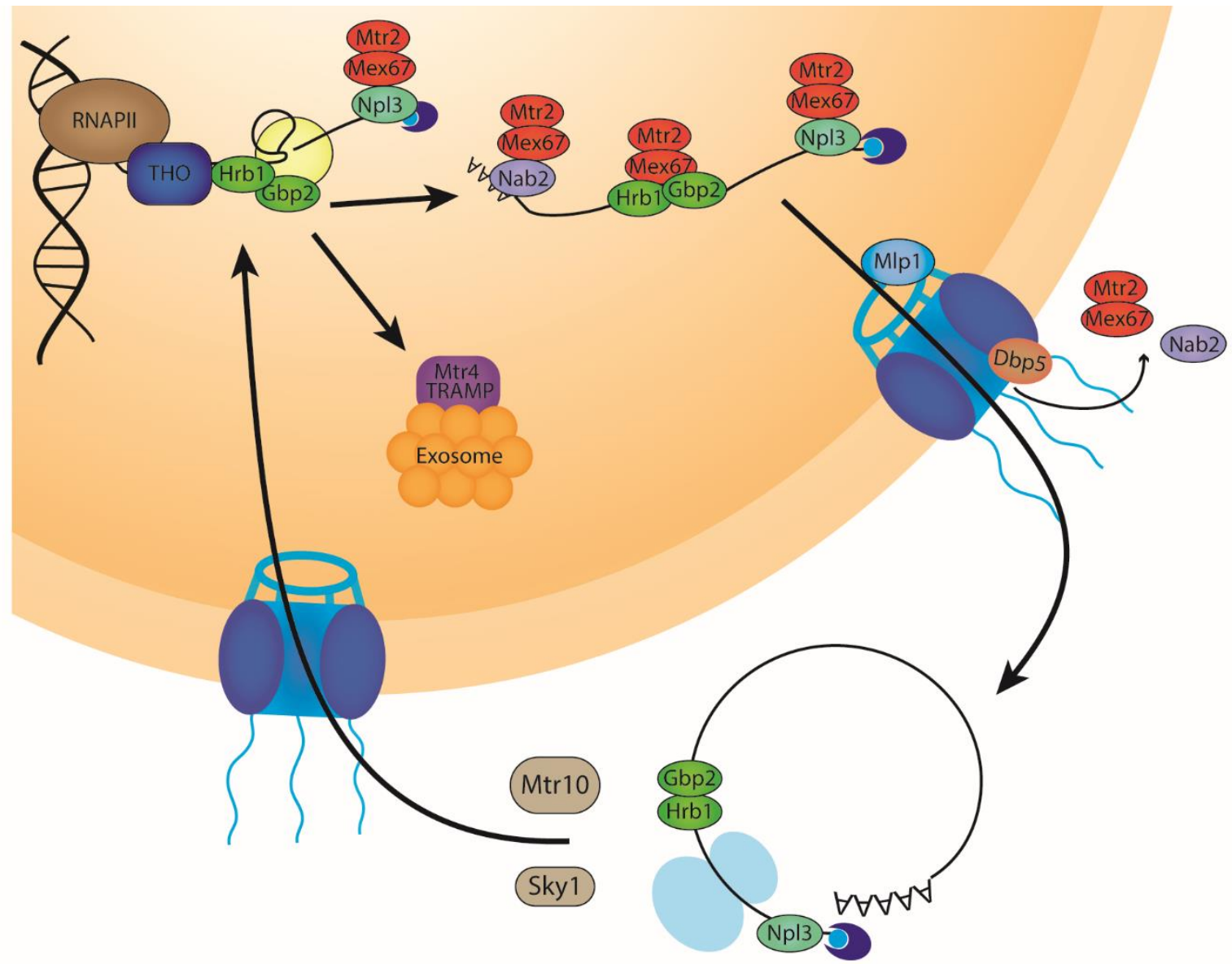

Figure 2: Nucleo-cytoplasmic shuttling of Gbp2 and Hrb1

Gbp2 and Hrb1 bind to the late spliceosome and can induce RNA degradation via Mtr4 and the exosome or allow mRNA export via Mex67. They shuttle into the cytoplasm and in contrast to Mex67 and the guard protein Nab2, they stay bound on the mRNA until translation. Their re-import is mediated by the kinase Sky1 and the karyopherin Mtr10. 
recruitment factor Yra1, it forms the TREX-(Transcription Export)-complex, which associates with Gbp2 and Hrb1. The association of Gbp2 and Hrb1 with intronless transcripts and the TREX complex indicates that they might be implicated in mRNA export for all mRNAs. However, overexpression of Gbp2 causes a toxic retention of bulk mRNA in the nucleus (Windgassen and Krebber, 2003), thus they appear to restrict export rather than promoting it.

When the mRNA is exported, Gbp2 and Hrb1 shuttle into the cytoplasm. While the Mex67 binding proteins Yra1 and Nab2 dissociate from the mRNP before and immediately after export, respectively (Iglesias et al., 2010; Stewart, 2010), the three SR-like proteins Gbp2, Hrb1 and Npl3 stay bound until translation (see Figure 2; Windgassen et al., 2004). Npl3 was identified as a translation inhibitor and might have a role in termination accuracy (Estrella et al., 2009; Rajyaguru et al., 2012; Windgassen et al., 2004). However, the cytoplasmic function of Gbp2 and Hrb1 is, to date, entirely unknown.

\subsection{Translation}

\subsubsection{Translation initiation and elongation}

In the cytoplasm, the CBC is replaced with elF4E at the 5' cap. It is not clear how the cap exchange is regulated. The $\mathrm{CBC}$ can also interact with the initiation factor elF4G and promote translation. The elF4E mediated translation was, however, found to be more effective (Fortes et al., 2000). In higher eukaryotes, early translation is presumably always CBC associated (Ishigaki et al., 2001; Lejeune et al., 2002). In yeast, it is unknown when the cap binding structures are exchanged, but elF4E is the main cap binder during translation in eukaryotes (Gingras et al., 1999). elF4E interacts with elF4G and this interaction is essential for translation and cell growth (Ptushkina et al., 1998). There are two isoforms: elF4G1 and elF4G2. The relevance is not clear, as they appear to be functionally redundant (Clarkson et al., 2010). The translation initiation factors elF4E and elF4G are target of several translational repressors, such as the elF4E binding proteins Caf20 and Eap1 (Ibrahimo et al., 2006; Park et al., 2006) or the elF4G binding proteins Sbp1, Scd6 and Npl3 (Rajyaguru et al., 2012). elF4G is a scaffold, which interacts with several proteins during translation. Its interaction with the $\operatorname{poly}(\mathrm{A})$ binding protein $\mathrm{Pab} 1$, allows circularisation of the mRNA (Wells et al., 1998). While the circularisation generally requires elF4E, elF4G, elF3 and Pab1, a second state in association with an 80S ribosome was described to require the termination factors eRF1 and eRF3 (Amrani et al., 2008). The circularisation is thought to promote translation and mRNA stability, however, it does not appear to be essential for cell growth (Park et al., 2011). elF4G and elF4B promote the binding of the preinitiation complex to the mRNA to initiate translation (see Figure 3).

For translation elongation, eEF1A-GTP delivers the tRNA to the P-site. Hybridisation of 
codon and anti-codon induce conformational changes, which lead to GTP hydrolysis and release of eEF1A-GDP. eEF2-GTP induces the ribosome translocation through GTP hydrolysis and eIF5A promotes the peptide bond formation. eEF3, which was only found in fungi, likely promotes tRNA release at the E-site. (Translation elongation is reviewed in Dever et al., 2016).

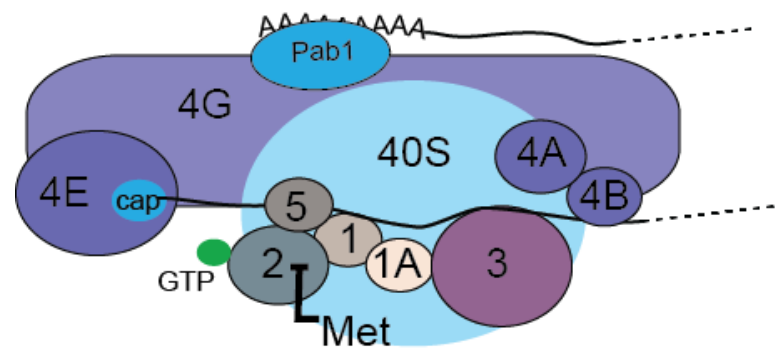

\section{Figure 3: Cap dependent translation initiation}

The preinitiation complex (PIC) consists of the $40 \mathrm{~S}$ ribosomal subunit, elF1, elF1A, elF3, elF5 and the ternary complex of eIF2, GTP and the initiator tRNA. The PIC binds to the 5' UTR. It is in an open conformation, the anticodon of the initiator tRNA is not hybridised with the mRNA. The DEAD box helicase elF4A promotes PIC recruitment and movement. Its binding to elF4G increases the elF4A ATPase activity. elF4B promotes the elF4G-elF4A interaction. The small ribosomal subunit scans the mRNA until it reaches the start AUG. elF2 hydrolyses GTP and elF2, GDP, elF1 and elF5 are released. That leads to conformational changes and the PIC assumes a closed position. elF5B (not depicted) hydrolyses GTP for joining of the 60 S ribosomal subunit. Release of elF1A and elF5B-GDP forms the $80 \mathrm{~S}$ ribosome. The initiation tRNA is in the P-site, the E- and A-sites are vacant. Translation initiation is reviewed in Dever et al., 2016 and the figure was adapted from there.

\subsubsection{Translation termination}

The three different termination codons are recognised by eRF1, which is structurally similar to tRNAs (Frolova et al., 1994; Jacobson, 2005). The GTPase eRF3 induces structural rearrangements of eRF1. This moves a conserved GGQ motif of eRF1 into the peptidyl transferase centre of the terminating ribosome. This induces hydrolysis and release of the polypeptide (Alkalaeva et al., 2006).

This process is orchestrated by several different proteins. Rli1 is required for functional stop codon recognition. It interacts with eRF1 and eRF3 and promotes termination by a mechanism that requires its iron sulphur cluster (Khoshnevis et al., 2010; Shoemaker and Green, 2011). Rli1 is an ATP binding cassette (ABC) protein. ATPase activity appears to 
be required for ribosome recycling (see below) but not for translation termination. It was originally suggested that it might act after dissociation of eRF3 to promote the peptide release function of eRF1 (Preis et al., 2014). However, recent analyses indicate that Rli1 and eRF3 might bind to the terminating ribosome first. Rli1 might promote the binding of eRF1, which appears to be delivered by the helicase Dbp5 (see Figure 4; Beißel et al., 2019). Dbp5 was shown to promote stop codon recognition and it requires its ATPase

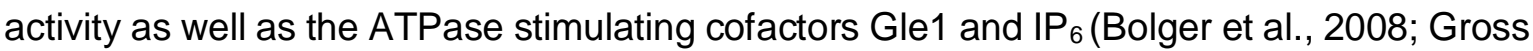
et al., 2007). Dbp5 seems to be relevant for the correct positioning of eRF1. Only after the ATP hydrolysis and dissociation of Dbp5, eRF1 can interact with eRF3. Although Dbp5 seems to prevent the interaction of eRF1 to eRF3 initially, its control over this step leads to a more stable association of eRF1 and eRF3 with the ribosome (Beißel et al., 2019). The initiation factor elF3, and most importantly its subunit Hcr1 (elF3j) is also involved in translation termination. Likely, Hcr1 promotes the dissociation of eRF3-GDP after it has induced structural rearrangements in eRF1 (Beznosková et al., 2013).

The interaction of Pab1 with eRF3 promotes efficient translation termination (Cosson et al., 2002; Roque et al., 2015). The termination efficiency is further modulated by additional factors (Czaplinski et al., 2000; Urakov et al., 2001, 2017).

After release of the peptide, and most likely eRF3, Rli1 actively splits the ribosome, which requires the hydrolysis of ATP (Barthelme et al., 2011; Pisarev et al., 2010). In addition to Rli1, the dissociation and recycling of the post-termination ribosome complex requires the initiation factors elF1, elF1A, and elF3 (Pisarev et al., 2010).

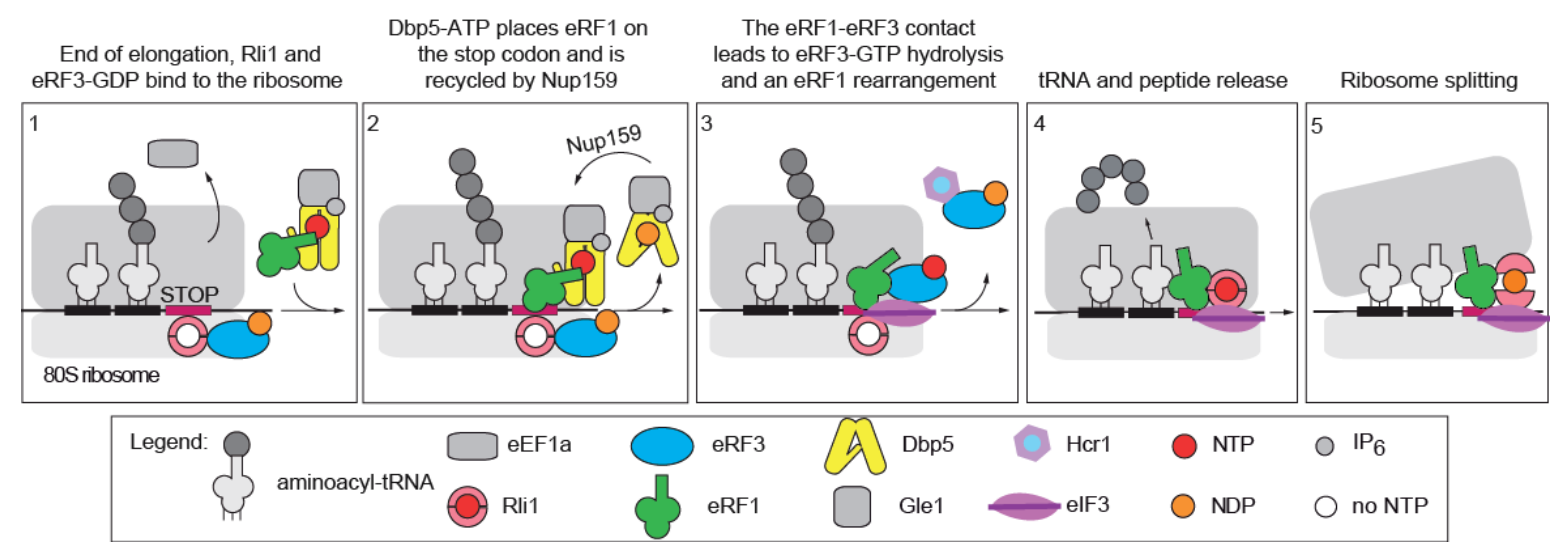

Figure 4: Translation termination

(Adapted from Beißel et al., 2019) 


\subsection{Cytoplasmatic mRNA degradation}

The degradation of mRNAs in the cytoplasm typically starts with shortening of the poly $(A)$ tail (Garneau et al., 2007). To initiate degradation, the poly(A) tail is shortened by specific exonucleases. The heterodimer Pan2-Pan3 can degrade the poly $(A)$ tail and, interestingly, it is dependent on Pab1 (Boeck et al., 1996; Brown et al., 1996). The main deadenylation machinery in yeast is the CCR4-NOT complex. (Liu et al., 1998; Tucker et al., 2001). The catalytic subunit Ccr4 is inhibited by Pab1 in vitro, suggesting that Pab1 has to dissociate prior to CCR4-NOT deadenylation (Tucker et al., 2002). It was suggested that Pan2-Pan3 are rather involved in trimming the poly $(A)$ tail to an mRNA specific length (Brown and Sachs, 1998). In mammalian cells it was shown, that PAN2-PAN3 perform an initial trimming, followed by a second CCR4-NOT deadenylation phase (Yamashita et al., 2005). Higher eukaryotes also have a cap dependent deadenylation complex called PARN (poly(A)-specific ribonuclease; Dehlin et al., 2000). The mRNA is either degraded through 3'-5' degradation by the cytoplasmic exosome, or through 5'-3' degradation by the cytoplasmic Rat1 paralogue Xrn1 (see Figure 5; Garneau et al., 2007). Both pathways are not mutually exclusive and appear to be redundant (He et al., 2003; Houalla et al., 2006).

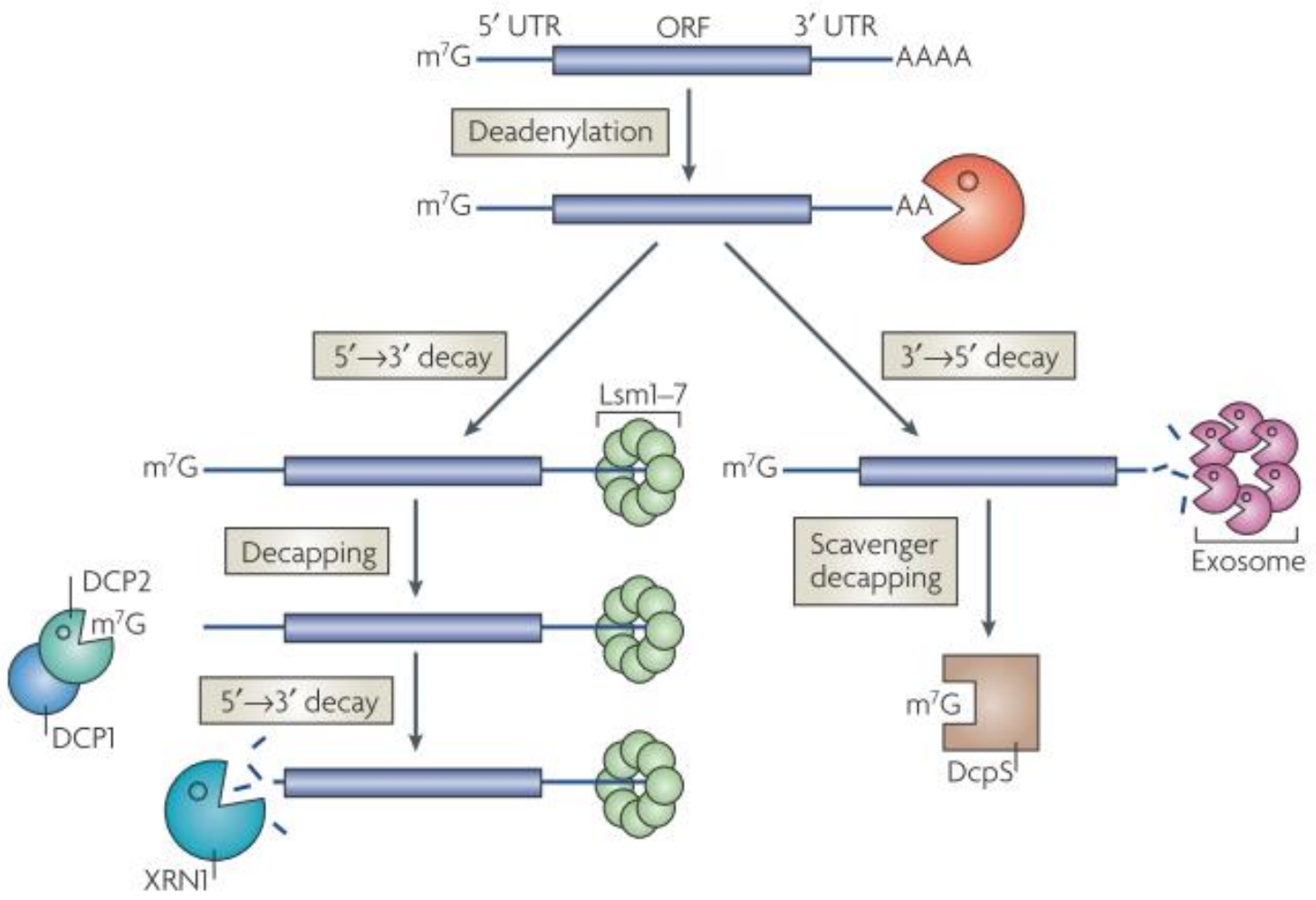

\section{Figure 5: Deadenylation dependent decay}

Normal cytoplasmic decay starts with deadenylation and has two pathways: exosomal 3'-5' decay or decapping and 5'-3' decay by Xrn1. The decapping holoenzyme requires the Lsm1-7 ring for recruitment. (Adapted from Garneau et al., 2007) 
The cytoplasmic exosome requires a different cofactor than its nuclear counterpart - the Ski complex. Ski2, Ski3 and two Ski8 molecules form a heterotetramer (Brown et al., 2000; Synowsky and Heck, 2008). Interestingly, the helicase Ski2 has a high structural similarity to its nuclear counterpart Mtr4 (Halbach et al., 2012). The factor Ski7 links the Ski2/3/8 tetramer to the exosome and is required for degradation (Araki et al., 2001). After deadenylation, the exosome can degrade the mRNA (Houseley et al., 2006). The remaining capped 5' fragment requires the scavenger decapping enzyme Dcs1/DcpS for degradation (Liu et al., 2002). Interestingly, Dcs1 improves the RNA affinity of Xrn1, indicating that it is also a cofactor for the 5'-3' degradation pathway (Sinturel et al., 2012).

For 5'-3' degradation, the cap has to be removed, as Xrn1 requires an exposed 5' phosphate for degradation (Jinek et al., 2011). The cap is removed by the decapping holoenzyme Dcp1-Dcp2, with Dcp2 containing the enzymatic activity (Steiger et al., 2003). Normal mRNA decapping is also deadenylation dependent (Decker and Parker, 1993; Hsu and Stevens, 1993; Muhlrad et al., 1994). Pab1 was shown to inhibit decapping and this inhibition is partially, but not completely, mediated by the Pab1-elF4E interaction (Caponigro and Parker, 1995; Wilusz et al., 2001). The decapping enzyme requires at least the first 25 nucleotides at the 5' end of the mRNA for binding (LaGrandeur and Parker, 1998). Dcp1 can interact with elF4G and the elF4E-elF4G-Pab1 complex, but it competes with elF4E for cap binding (Ramirez et al., 2002; Schwartz and Parker, 2000; Vilela et al., 2000). The decapping enzyme requires a ring-structure of the Sm-like proteins Lsm1-7. The Lsm-ring promotes binding of the decapping enzyme to an mRNA. It does not bind to mRNAs, which are associated with elF4E and Pab1 (Tharun and Parker, 2001; Tharun et al., 2000). Likely, earlier factors already promote the commitment of an mRNA to degradation, leading to the dissociation of translation factors. Several factors are known to promote mRNA decapping: the enhancers of decapping Edc1, Edc2 and Edc3 (Kshirsagar and Parker, 2004; Schwartz et al., 2003), the DEAD-box helicase Dhh1 (Coller et al., 2001), the elF4G binding proteins Sbp1 and Scd6 (Fromm et al., 2012; Rajyaguru et al., 2012; Segal et al., 2006) and Pat1. Pat1 is possibly the earliest factor as it seems to bind mRNAs already during translation (Bonnerot et al., 2000). The competition between decapping and translation is further highlighted by the fact that the decapping promoting factors Sbp1, Scd6, Dhh1 and Pat1 also inhibit translation (Coller and Parker, 2005; Rajyaguru et al., 2012). Translation inhibition and the 5'-3' decay pathway are associated with distinct cytoplasmic foci - the P-(processing)-bodies (Eulalio et al., 2007; Sheth and Parker, 2003). P-bodies are dynamic structures, that are enriched when the 5'-3' pathway is overloaded. Mutants of the decapping enzyme or Xrn1 increase the number and size of P-bodies, while mutant of earlier promoting factors reduce the size and number of P-bodies (Garneau et al., 2007). Interestingly, it was observed that translationally inhibited mRNAs can be released 
from P-bodies to re-enter translation. Hence, they might also regulate protein synthesis under certain conditions by sequestering mRNAs until they are needed again (Brengues et al., 2005). Exosome subunits were also found in cytoplasmic foci; however, it is unclear if these are related to P-bodies (Garneau et al., 2007; Graham et al., 2006).

Interestingly, in addition to the normal deadenylation dependent 5'-3' decay pathway, deadenylation independent pathways exist. Rps28A binds to the 3 ' end of its own mRNA and recruits Edc3. This induces deadenylation independent decay (Badis et al., 2004). The EDC1 mRNA can undergo deadenylation independent decapping in a mechanism which involves Not2, Not4 and Not5 of the CCR4-NOT complex (see above) (Muhlrad and Parker, 2005). The most prominent deadenylation independent decay pathway is most likely nonsense mediated decay.

\subsection{Nonsense Mediated Decay}

An mRNA that has reached the cytoplasm and entered translation is still subject to quality control. Nuclear mRNA quality control is based on the correct RNA processing (see 2.1 and 2.2). In the cytoplasm, mRNAs are checked based on their open reading frame and translatability. A correct mRNA typically has a start codon close to the 5' end, an ORF that allows efficient elongation, and a stop codon close to the 3 ' end. These features are controlled by three different surveillance mechanisms, which can induce rapid mRNA decay. No-Go Decay (NGD) targets mRNAs, on which the ribosome is stalled during elongation. This phenomenon typically occurs at strong secondary structures or rare codons. Also broken mRNAs, where the ribosome reaches a 3' end without a stop codon or poly(A) tail, are NGD targets (Shoemaker et al., 2010). No-Stop Decay (NSD) targets mRNAs that lack a termination codon - typically as a consequence of cryptic polyadenylation signals. On NSD targets, the ribosome translates the poly $(A)$ tail into a poly lysine stretch and stalls at the end of the mRNA without translation termination (Klauer and van Hoof, 2012). The main factors for NGD and NSG are Dom34 and Hbs1. These factors are structurally similar to eRF1 and eRF3, they likely enter the A-site of stalled ribosomes and induce mRNA decay (Graille and Séraphin, 2012; Klauer and van Hoof, 2012; Shoemaker et al., 2010). The nascent polypeptide is actively pulled out of the ribosome and immediately ubiquitylated by a mechanism called Ribosome Associated Quality Control (Inada, 2017).

Nonsense Mediated Decay (NMD) targets mRNA, on which translation terminates prematurely. NMD substrates typically have a Premature Termination Codon (PTC). A major group of NMD substrates are unspliced pre-mRNAs that escape the nuclear quality control and leak into the cytoplasm. The retained intron sequences typically contain PTCs. Interestingly, short introns are under selective pressure to contain an in-frame PTC (Jaillon 
et al., 2008). This probably reflects that NMD acts as a fail-save mechanism for the nuclear splicing quality control (He et al., 1993, 2003). In higher eukaryotes, NMD is also coupled to alternative splicing. Some exons contain premature termination codons. Inclusion of a PTC containing exon in the mRNA sequence causes NMD. Thus, through the choice of the splice site, the cell can regulate gene expression (McGlincy and Smith, 2008). NMD also targets short upstream open reading frames (uORFs), pseudogenes, transposable elements and several classes of non-coding RNAs (Celik et al., 2017; He et al., 2003; Thompson and Parker, 2007; Welch and Jacobson, 1999). The level of some non-coding RNAs are regulated by addition of 3' extensions, which are targeted by NMD. It is, however, not clear if these transcripts also enter normal translation or a different process (Marquardt et al., 2011; Wery et al., 2016). Normal mRNAs can also be targeted by NMD. These mRNAs are typically poorly translated and often have a low codon optimality. Normal mRNAs that are sensitive to NMD are thought to be more prone to frameshifting or bypassing the initiator AUG. Some wild typical mRNAs that are destabilised by NMD are low abundant isoforms caused by alternative transcription sites (Celik et al., 2017; Johansson et al., 2007). NMD was implicated in telomere maintenance and kinetochore regulation (Dahlseid et al., 1998; Lew et al., 1998), but it is unlikely that NMD plays an important role as the disruption of the NMD pathway causes no growth defect in S. cerevisiae (Cui et al., 1995; He and Jacobson, 1995; Leeds et al., 1991). In higher eukaryotes, NMD is also involved in removing unproductive products of genomic rearrangements in lymphocytes (Wang et al., 2002a) and is essential in certain developmental stages (Medghalchi et al., 2001; Metzstein and Krasnow, 2006; Yoine et al., 2006).

\subsection{UPF proteins - the main NMD factors}

The activation of NMD requires translation. It was shown that inhibiting translation also reduces the Nonsense Mediated Decay. These reports describe either mutants of the translation machinery or addition of cycloheximide, a drug that inhibits translation elongation (Peltz et al., 1992; Zhang et al., 1997; Zuk and Jacobson, 1998). This finding is corroborated by the fact that NMD occurs on polysomes (mRNAs that are engaged with several ribosomes; Atkin et al., 1997; Hu et al., 2010; Zhang et al., 1997). On uORFs it was observed that efficient NMD correlates with a high ribosome occupancy (Gaba et al., 2005). But more precisely, NMD can only be activated after a stop codon is recognised by the release factors eRF1 and eRF3 (Amrani et al., 2006; Kervestin and Jacobson, 2012). Nonsense suppression also suppresses NMD (Keeling et al., 2004).

NMD is induced by the assembly of three Upf proteins at a ribosome that terminated prematurely (Kervestin and Jacobson, 2012). Upf1 has a helicase domain composed of two 
RecA-like domains and two regulatory domains called $1 \mathrm{~B}$ and $1 \mathrm{C}$. It exhibits RNA dependent ATPase and helicase activity (Altamura et al., 1992; Chakrabarti et al., 2011; Czaplinski et al., 1995). It also has a cysteine histidine rich $(\mathrm{CH})$ domain, which is required for interaction with the C-terminal domain of Upf2 (Altamura et al., 1992; Dehecq et al., 2018; He and Jacobson, 1995; Leeds et al., 1992). Upf2 connects Upf1 with Upf3, it has three MIF4G domains, and one of these interacts with Upf3 (He and Jacobson, 1995; He et al., 1997; Mendell et al., 2000). Upf2 requires Up3 but not Upf1 for polysomal association (Atkin et al., 1997). Upf3 has nuclear localisation signals (NLSs) as well as a nuclear export signals (NESs) and was shown to move between nucleus and cytoplasm (Shirley et al., 1998). Upf1 is thought to be the main factor of NMD, as its overexpression can supress the loss of Upf2 and Upf3, at least for some aspects of the pathway, but not vice versa (Maderazo et al., 2000). Binding of Upf2 and Upf3 to Upf1 increases its ATPase and helicase activity, which promotes NMD. Structural analysis revealed that free Upf1 has a closed conformation. Its $\mathrm{CH}$-domain interacts with its helicase domain. In this conformation it has higher binding affinity to RNA (Chakrabarti et al., 2011). When Upf2 contacts Upf1, structural rearrangement move the $\mathrm{CH}$-domain, which reduces the RNA binding and increases the ATPase and helicase activity. Thus, binding of Upf2 and Upf3 switches Upf1 from RNA binding to an RNA unwinding activity (Chakrabarti et al., 2011). Due to their low abundance, it is likely that Upf2 and Upf3 do not interact with all mRNPs but might rather be specifically recruited to NMD substrates (Dehecq et al., 2018; Maderazo et al., 2000). Upf1 is thought to interact with all mRNAs, mostly in the 5' UTR, but it has a higher affinity for NMD substrates (Johansson et al., 2007). It was shown that Upf1 interacts with the $40 \mathrm{~S}$ ribosomal subunit via its $\mathrm{CH}$-domain (Min et al., 2013). Upf1 can interact with both eRF1 and eRF3. Upf2 and Upf3 can also interact with eRF3, but in contrast to Upf1, they compete with eRF1 for eRF3 binding (Czaplinski et al., 1998; Wang et al., 2001) The eRF1-eRF3 binding to Upf1 reduces its ATPase and helicase activity (Czaplinski et al., 1998). Thus, it was proposed that Upf1 might be recruited first in an inactive mode, followed by activation through Upf2-Upf3 binding (Kervestin and Jacobson, 2012). As all three Upf proteins are dispensable for growth under normal conditions, it is unlikely that they have a particular relevance for normal translation termination (Amrani et al., 2006). It was observed that loss of the Upf proteins leads to nonsense suppression (Wang et al., 2001). It was suggested that this might be an indirect effect of MND causing an altered magnesium uptake by targeting the mRNA of a magnesium transporter (Johansson and Jacobson, 2010). However, Weng et al., 1996 identified UPF1 mutants that cause nonsense suppression but have no apparent defect in degradation of NMD substrates. 


\subsection{Premature termination}

The Upf proteins assemble at the terminating ribosome to initiate NMD. But for the pathway to function correctly, these factors need to be recruited selectively to translation termination that occurs prematurely. It was studied extensively how the translation and surveillance machinery can distinguish a Premature Termination Codon (PTC) from a Normal Termination Codon (NTC). One model that was proposed is the faux-UTR model. It is based on the observation that extending the 3' UTR of an mRNA converts it into an NMD substrate (Muhlrad and Parker, 1999a). Hence, it was suggested that the great distance from a PTC to the poly $(A)$ tail creates an unnaturally long 3' UTR, which is sensed by the cell. A key factor in this model is the poly (A) binding protein Pab1. An NTC is in close proximity to the poly $(\mathrm{A})$ tail. Pab1 can interact with eRF3, and promote normal translation termination (Cosson et al., 2002). When termination occurs at a PTC, Pab1 is not in proximity to the termination complex, which likely allows binding of Upf1 (Brogna and Wen, 2009). The importance of Pab1 for PTC definition was highlighted by the finding that tethering Pab1 downstream of a PTC prevents NMD and induces regular termination (Amrani et al., 2004). It was assumed that Pab1 might inhibit the Upf1 binding to eRF3. However, in vitro experiments showed that Upf1 reduces Pab1 binding to eRF3, but the Upf1 binding is unaffected by Pab1. It was suggested that the function of Pab1 might be more complex (Kervestin et al., 2012). The competition of Pab1 and Upf1 might also require the correct mRNP context (Kervestin and Jacobson, 2012). This underlines that other factors must be involved in the PTC distinction. Indeed, Pab1 is known to be a scaffold for regulatory proteins (Mangus et al., 2003), thus, other factors might affect translation termination in a Pab1 dependent manner (Amrani et al., 2006).

However, the absence of Pab1 or the poly $(A)$ tail does not induce NMD at NTCs (Meaux et al., 2008). Thus, also Pab1 independent mechanisms must be involved in the definition of a PTC. In fact, not only Pab1 tethering prevents NMD but also cloning a stretch of normal 3' UTR sequence downstream of a PTC (Amrani et al., 2004). It is known that regulatory factors bind to the 3' UTR and regulate termination and degradation (Wilkie et al., 2003; Wilson and Brewer, 1999). Thus, such regulatory factors might also affect NMD. This finding fits to a second model of NMD regulation - the marking model. A PTC does not only differ from an NTC in its distance to the poly (A) tail. A PTC is also followed by the remaining ORF sequence, instead of the regular 3' UTR sequence. It was proposed that proteins might mark the open reading frame and 3' UTR. Thus, a prematurely terminating ribosome would be in proximity to the "wrong" protein context (Kervestin and Jacobson, 2012). In the early NMD research, several sequence elements were found to influence NMD. Sequences were discovered that might mark an ORF. These sequences could induce NMD only when they were cloned downstream of a termination codon. Hence, they were called Downstream 
Sequence Elements (DSEs; Peltz et al., 1993; Ruiz-Echevarria and Peltz, 1996; RuizEchevarría et al., 1998; Zhang et al., 1995). It was suggested that regulatory factors bind to these sequence elements and are removed by the translating ribosome. If the DSE is downstream of a termination codon, the ribosome does not remove the regulatory protein, which might induce NMD as a consequence. The protein Hrp1 was indeed found to bind to a DSE and induce NMD if located 3' of a termination codon (González et al., 2000). It interacts with Upf1 and Upf2. Interestingly, the Hrp1-Upf2 interaction is apparently direct, but promoted by Upf1 and RNA (Wang et al., 2006).

Conversely, the uORFs of the GCN4 and the YAP1 mRNA were found to be followed by stabilising sequences preventing NMD (Ruiz-Echevarría and Peltz, 2000; Ruiz-Echevarría et al., 1998). It was shown that such a sequence can prevent NMD if it is located downstream of a PTC and was called Stabilizer Element (STE). A different type of STE was shown to inhibit NMD if located upstream of a termination codon (Hagan et al., 1995; Peltz et al., 1993). The identified STEs bind the protein Pub1, which they require for the stabilisation effect.

The mechanisms for Hrp1 or Pub1 mediated NMD regulation are not understood. However, these experiments show, in principal, that ORFs and 3' UTR could be bound by regulation factors that either prevent or induce NMD (González et al., 2001a). Also, in higher eukaryotes, mRNAs that naturally have long 3' UTRs were shown to contain stabilising sequence elements to prevent NMD (Toma et al., 2015). NMD is known to have a certain polarity, i.e. a PTC can induce NMD more strongly if it is located farther from the 3' end and closer to the 5' end (Cao and Parker, 2003; Losson and Lacroute, 1979). This agrees with the faux-UTR model, which defines the distance of the termination codon to the poly $(A)$ tail as the main determinant for NMD. However, the polarity is not linear, indicating that there are discreet positions in an mRNA that influence NMD (Yun and Sherman, 1995). This suggests that both the length of the 3' UTR and the mRNA sequence have an influence on NMD initiation (see Figure 6). However, the identified sequence elements are not very conserved between different mRNAs (Amrani et al., 2006). This argues against the idea of distinct motifs that regulate NMD. The identified elements were, however, clearly sequence dependent. It is unknown how many different sequence elements might exist or how many different proteins may be involved in such a regulation.

Surprisingly, mRNAs with long ORFs were shown to escape NMD (Decourty et al., 2014), suggesting that there is an additional mode of regulation, such as the distance to the initiator AUG. Likely, a PTC is defined by an interplay of several different regulatory elements.

It was shown that premature termination is less efficient than regular termination, even in the absence of Upf1 (Amrani et al., 2004). The ribosome appears to pause at the PTC, as discovered by ribosome foot-printing. On an NTC this was only observed in an eRF1 mutant. 
The inefficient translation termination is likely caused by the absence of promoting factors and/or the presence of inhibiting factors. This notion fits both the faux-UTR and the marking model but suggests that the protein context may not induce or prevent NMD directly. The protein context at a PTC might decrease the termination efficiency and the paused ribosome might present a binding site for Upf1. Such a model would not disagree with the notion that proteins might additionally promote or inhibit Upf1 recruitment directly. It might be an additional regulation of selective Upf1 recruitment (Kervestin and Jacobson, 2012). The release factor eRF3 likely dissociates from the ribosome before ribosome recycling starts (Becker et al., 2012). An inefficient release of eRF3 at the PTC was suggested as a possible Upf1 recruitment mechanism (Kervestin and Jacobson, 2012). Upf1 might not be able to

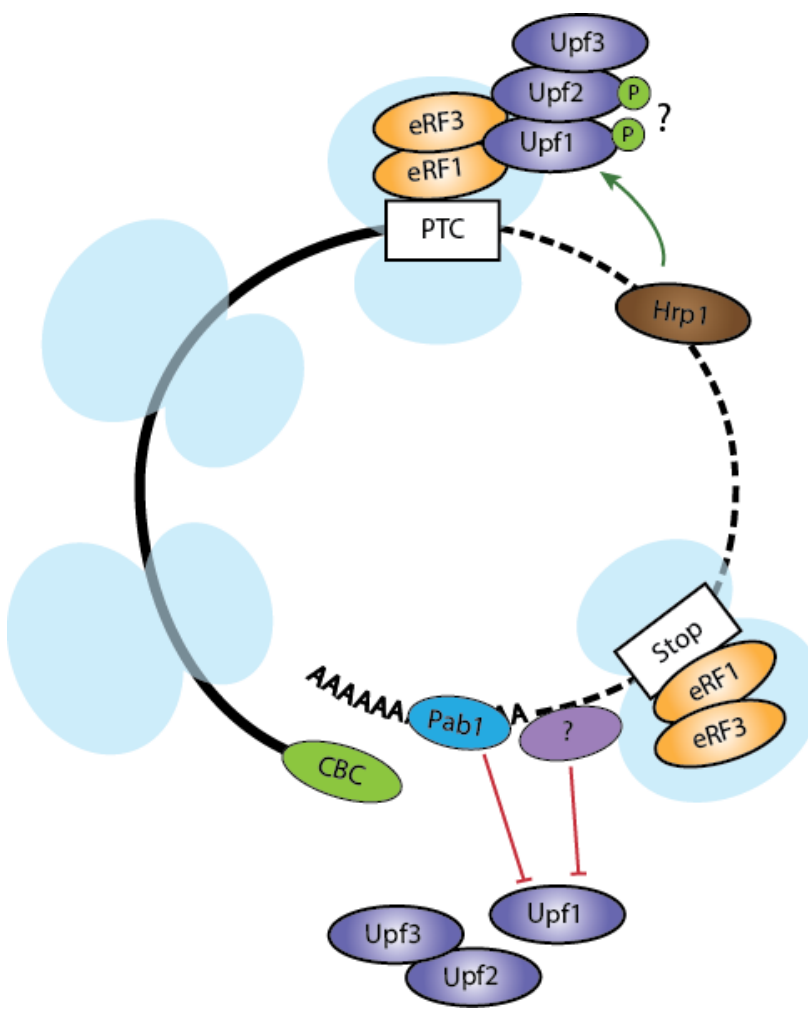

Figure 6: NMD initiation in S. Cerevisiae

The cell can differentiate a normal stop codon from a PTC. At a normal stop codon Pab1 and likely factors of the 3' UTR promote normal termination and inhibit NMD. A PTC causes an aberrant 3' UTR, which is extended in length and likely has the wrong protein context. The distance to Pab1 allows the formation of the Upf1-2/3 complex at the terminating PTC, which initiates NMD. This is further influenced by sequence elements. Hrp1 induces NMD if bound to a sequence element downstream of a stop codon. A similar mechanism is known for the Exon Junction Complex in higher eukaryotes (see 2.9). In higher eukaryotes, NMD initiation also requires Upf1 and Upf2 phosphorylation, which was reported but is not completely clear in yeast (Wang et al., 2006). 
interact with eRF3 if translation termination and eRF3 release are efficient. However, tethering eRF3 to a PTC inhibits NMD rather than promoting it (Amrani et al., 2004), thus, the mechanism must be more complex. Available eRF3 doesn't simply recruit Upf1 to the ribosome to induce NMD, but it would still be possible that eRF3-GDP in the P-site is a specific recruitment platform for Upf1.

Other models were proposed that might influence NMD substrate stability and translatability in addition. One model describes that on an NMD substrate a long stretch of mRNA is not passed by translating ribosomes, due to the artificially increased 3' UTR. It was hypothesised that this stretch might expose the mRNA to increased degradation via the regular mRNA degradation pathways (Brogna and Wen, 2009). A different model focuses on the circularisation of mRNA. On a circular mRNA the poly $(A)$ tail is in close proximity to the 5' end. Ribosomes might be recycled at the termination codon and directly transferred to the 5' UTR for a new round of scanning and translation initiation. Such an efficient translation initiation might be impossible for NMD targets due to the extended distance between PTC and poly(A) tail (Brogna and Wen, 2009). Alternatively, it was also proposed that an initial round of translation might induce stabilising mRNP remodelling, but only if the ribosome reaches the normal end of the ORF - close to the 3' end of the mRNA (Amrani et al., 2006; Hilleren and Parker, 1999).

\subsection{Effects of NMD}

When a stop codon is recognised as premature and the Upf proteins assemble at the terminating ribosome, NMD is initiated. Upf1 appears to induce increased mRNA degradation, inhibition of translation, and dissociation of the ribosome from the PTC (Muhlrad and Parker, 1999b; Serdar et al., 2016). The fact that NMD also inhibits the translation of the aberrant mRNAs, underlines that NMD reduces the synthesis of aberrant and potentially toxic polypeptides. Further, the translation products of different PTCcontaining HIS3 constructs were shown to be targeted to proteasomal decay by the NMD pathway (Kuroha et al., 2009). Consistently, it was also shown that the $\mathrm{CH}$-domain of Upf1 likely functions as an E3 ubiquitin ligase. The domain also has some structural similarity to an E3 RING finger domain (Takahashi et al., 2008).

While the exact mechanism of the NMD pathway is largely unknown, some insight into the induction of accelerated mRNA decay could be gained so far. NMD targets were shown to be degraded predominantly by the 5'-3' pathway of decapping and Xrn1 catalysed RNA degradation (He et al., 2003; Muhlrad and Parker, 1994). PTC containing mRNAs were shown to accumulate in P-bodies. Interestingly, the localisation to P-bodies depends on Upf1 but is independent of its ATPase activity or Upf2-Upf3. (Sheth and Parker, 2006). In contrast to regular decapping in RNA decay, the NMD pathway is independent of 
deadenylation even though accelerated deadenylation was observed (Muhlrad and Parker, 1994). This might reflect that NMD occurs before the RNA is circularised or before it has assumed an mRNP conformation, in which the poly $(\mathrm{A})$ tail inhibits decapping. Alternatively, it may reflect that the NMD pathway uses a mechanism that circumvents the stabilising effect of the poly(A)-tail and Pab1.

However, deadenylation and 3'-5' decay by the exosome and the Ski complex were also reported for NMD targets. But the exosomal decay was described to be the minor degradation pathway (Mitchell and Tollervey, 2003). A decapping mutant (to inactivate the 5'-3' pathway) was used to analyse the role of 3'-5' decay in NMD.

It was recently described that Upf1 initially forms a complex with Upf2-Upf3 (the Upf1-2/3 complex) and then recruits the 5'-3' degradation machinery without Upf2-Upf3 (the Upf1decapping complex; Dehecq et al., 2018). It appears to mediate the assembly of a protein complex consisting of Upf1, Ebs1, Nmd4, Edc3, Dcp1, Dcp2 and Hrr25. The protein-protein interactions were found to be RNase insensitive, which was also previously reported for the interaction of Upf1 with Dcp1 (Ford et al., 2006). Upf1 interactions with Lsm1-7 were RNase sensitive (Dehecq et al., 2018), indicating that they are not part of the Upf1-decapping complex. Consistently, it was previously reported that the Lsm-ring and Pat1 are not required for degradation of PTC-mRNAs (Bouveret et al., 2000). Thus, NMD appears to recruit the degradation machinery in an alternative, Upf1 dependent, mechanism. However, Upf1 has an RNase resistant interaction with Pat1 (Dehecq et al., 2018), thus the role for Pat1 is not clear. Xrn1 also showed RNase resistant interaction with Upf1 (Dehecq et al., 2018). Hence, it might also be specifically recruited to NMD substrates to allow immediate degradation once the mRNA has been decapped. These interaction data are consistent with published data from a yeast two hybrid analysis. The two-hybrid analysis showed direct interactions of Upf1 with Upf2, Edc3 and Pat1. Interactions with Edc3 and Pat1 appeared to be Upf2 independent. The Upf1-Dcp1 interaction was strongly diminished in the absence of Edc3 (Swisher and Parker, 2011).

Upf1 was also shown to form an RNase resistant and Upf2 dependent interaction with Ski7, indicating that Upf1 may recruit the 3'-5' degradation machinery in a similar manner. (Takahashi et al., 2003)

\subsection{NMD in higher eukaryotes}

Although most of the NMD pathway is conserved through different species, there are some known mechanistic differences in human cells and other multi-cellular organisms compared to yeast. While DSEs are apparently absent in higher eukaryotes, there is a surprisingly similar mechanism.

During splicing, the Exon Junction Complex (EJC) is loaded $20-24$ nucleotides upstream 
of the exon junction (Le Hir et al., 2000). The EJC core consists of Y14, MAGOH, BTZ and elF4III (Isken and Maquat, 2007). A similar complex is not known in yeast. The EJC is involved in several functions including splicing (Blencowe et al., 1998; Fukumura et al., 2016; Mayeda et al., 1999), 3' end formation (Wiegand et al., 2003), stimulation of translation (Gudikote et al., 2005; Nott et al., 2004). Interestingly, the EJC associates with UAP56 and REF/ALY (the orthologues of Sub2 and Yra1, respectively) of the TREX complex (Singh et al., 2012). Thereby the EJC might link splicing to nuclear mRNA export (Maquat, 2004) surprisingly similar to Gbp2 and Hrb1 in yeast (see 2.2). Further, the EJC also associates with SR and SR-like proteins - most notably the SR-like proteins SRm160 and RNPS1 (Le Hir et al., 2001; Singh et al., 2012).

If an exon junction is at least $50-55$ nucleotides downstream of a termination codon, it induces NMD (Isken and Maquat, 2007; Lykke-Andersen et al., 2001). Wild typical mRNAs normally have the termination codon in the last exon. Thus, ECJs are not present in regular 3' UTRs. The EJC marks an open reading frame similar to the identified DSEs in yeast. Further, UPF3 associates with the EJC in the nucleus and is joined by UPF2 in the cytoplasm (Le Hir et al., 2001; Kim et al., 2017; Lykke-Andersen et al., 2001). When NMD is initiated, UPF1 interacts with the release factors and two additional factors: SMG8-SMG9. This complex is called the SURF (SMG UPF1 release factors) complex (Yamashita et al., 2009). The EJC presumably promotes the recruitment of UPF2-UPF3 to the SURF complex if an exon junction is downstream of the PTC. After UPF2-UPF3 binding, the kinase SMG1 phosphorylates UPF1, which increases the ATPase and helicase activity. This complex is called the DECID (decay inducing) complex (Kashima et al., 2006; Yamashita et al., 2009). The phosphorylated Upf1 interacts with SMG6 and SMG5-SMG7 (Okada-Katsuhata et al., 2012) to mediated degradation (see below). In addition to the EJC, the distance to the poly $(A)$ tail was also reported to be relevant for NMD in higher eukaryotes. Similar to yeast cells, PABP (the orthologue of Pab1) inhibits NMD (Eberle et al., 2008). This was tested with a construct that folds back at the 3 ' end to bring the PABP close to a PTC. Similar to tethering Pab1 in yeast, NMD was inhibited on this PTC.

There are two different NMD associated decay pathways in metazoans (Kervestin and Jacobson, 2012). One is initiated by association of the protein SMG6. SMG6 has a PIN domain with endonucleolytic activity. It can cleave the mRNA and the exposed 3' and 5' ends are degraded by the exosome and Xrn1 (Eberle et al., 2009; Huntzinger et al., 2008). The second pathway is mediated by SMG5 and SMG7. The SMG5-SMG7 pathway is independent of SMG6, and the mRNA is mostly degraded via decapping by DCP1-DCP2 and XRN1 degradation (Unterholzner and Izaurralde, 2004). SMG5 and SMG7 are localised in cytoplasmic decay bodies. Interestingly, tethering SMG7 to a transcript does not require Upf1 to induce degradation, showing that it acts downstream of NMD initiation (Unterholzner 
and Izaurralde, 2004). The SMG5-SMG7 degradation pathway might correspond to the degradation pathway in yeast. Interestingly the two yeast proteins Ebs1 and Nmd4 share sequence identity with the human SMG proteins. Nmd4 has a PIN domain, similar to SMG6 and SMG5 (Dehecq et al., 2018). As it was shown, in yeast, that a full-length PTC-containing mRNA is stabilised in the absence of Xrn1 (Serdar et al., 2016), thus, Nmd4 presumably has no endonuclease activity. Ebs1 has a 14-3-3 domain similar to SMG5, SMG6 and SMG7 and an HRR domain similar to SMG7 (Dehecq et al., 2018). Ebs1 was described to be localised in cytoplasmic foci. Its overexpression could suppress mutants of DCP1 (Ford et al., 2006). Loss of Ebs1 appears to have only a mild impact on the mRNA level of NMD targets (Ford et al., 2006). However, genome-wide analysis showed that transcript enrichment in ebs $1 \Delta$ cells correlates with $n m d 4 \Delta$ cells, and both knockout strains showed an overlap with transcripts enriched in upf1 $\Delta$ cells (Dehecq et al., 2018). Ebs1 was shown to generally repress translation, and NMD reporter translation was mildly enriched in ebs $1 \Delta$ $n m d 4 \Delta$ cells (Dehecq et al., 2018; Ford et al., 2006). The role of Ebs1 and Nmd4 is not clear in yeast, but the degradation pathway might be more conserved between yeast and human than originally assumed (Dehecq et al., 2018).

In human cells, SR-proteins were also implicated in NMD. The SR-related protein RNPS1 very likely acts in NMD via Upf2 as part of the EJC (Lykke-Andersen et al., 2001). At least three of the canonical SR proteins act as TAP (human orthologue of Mex67) adapters and shuttle into the cytoplasm. One of these, SRSF1, was shown to promote translation (Sanford, 2004). Further, its overexpression induces NMD on PTC-mRNAs. Although this could be an indirect consequence of increased translation (Sato et al., 2008), overexpressed SRSF1 does, however, not require shuttling to induce NMD and the nonshuttling SR proteins SRSF2 also induces NMD when overexpressed (Zhang and Krainer, 2004). It was discussed that NMD, at least in higher eukaryotes, might also occur in or directly at the nucleus (Maquat, 2004). Tethering SRSF1 downstream of a PTC enhances NMD, likely through increased UPF1 recruitment in or at the nucleus, which is enhanced by splicing and the EJC (Aznarez et al., 2018).

In higher eukaryotes, NMD is linked to the cap binding complex (CBC). The EJC is only found on CBC bound mRNA (Lejeune et al., 2002). Likely the first translating ribosome removes the EJCs from the transcripts. This led to the model of a pioneer round of translation, during which a transcript is susceptible to NMD. After the pioneer round the transcript may assume immunity. NMD was also described independent of the EJC, in a manner that reflects the 5'-3' polarity known from yeast NMD (Wang et al., 2002b), but the EJC appears to be a strong enhancer of NMD for most transcripts (Brogna and Wen, 2009). The pioneer round model was further corroborated by the finding that the CBC itself is involved in mRNP remodelling during NMD (Maquat et al., 2010). Thus, NMD likely targets 
only or mainly $\mathrm{CBC}$ bound transcripts during early translation. In yeast, however, the CBC appears to be dispensable for NMD (Gao et al., 2005) and transcripts never acquire immunity to NMD (Gaba et al., 2005; Keeling et al., 2004; Maderazo et al., 2000). Thus, NMD very likely also targets eIF4E bound transcripts in yeast cells. 


\subsection{Aim of the study}

Gbp2 and Hrb1 are known quality control factors that retain unspliced mRNA in the nucleus und recruit the degradation machinery (Hackmann et al., 2014). However, it is unknown, why they remain on the mRNA until translation. The cytoplasmic phase of their nucleocytoplasmic shuttling cycle is largely uncharacterised. But their nuclear re-import is regulated by the kinase Sky1 (Häcker and Krebber, 2004; Windgassen and Krebber, 2003). If their presence in the cytoplasm is regulated, they might fulfil a function, before they are ready to be imported into the nucleus to restart the cycle. Likewise, the third yeast SR-like protein, Npl3, has a very similar shuttling cycle and its re-import is also regulated by Sky1 (Windgassen et al., 2004). For Npl3 it was observed that it is involved in translation initiation and possibly termination accuracy (Baierlein et al., 2013; Estrella et al., 2009; Rajyaguru et al., 2012; Windgassen et al., 2004). In this study we tested the hypothesis that Gbp2 and Hrb1 might be involved in cytoplasmic quality control and we chose to investigate nonsense mediated decay. NMD degrades unspliced mRNAs that reach the nucleus, thus, it continues the surveillance function, which Gbp2 and Hrb1 already perform in the nucleus. In higher eukaryotes, splicing and NMD are connected by the Exon Junction Complex (Kervestin and Jacobson, 2012). Similar to the nuclear function of Gbp2 and Hrb1, the EJC was also implicated in connecting splicing and nuclear mRNA export via interactions with orthologues of Gbp2-Hrb1 interaction partners: Yra1 and Sub2 (REF/ALY and UAP56 in human; Maquat, 2004; Singh et al., 2012). Gbp2-Hrb1 and the EJC may also share the involvement in NMD. Further the EJC associates with SR-proteins and some human SR-proteins were already shown to influence NMD (Aznarez et al., 2018; Sato et al., 2008; Singh et al., 2012; Zhang and Krainer, 2004). However, their mechanism is not fully understood.

Taken together, it appears very likely that Gbp2 and Hrb1 also connect splicing and NMD in yeast. Further, the involvement of SR proteins in higher eukaryotes is very complex, since they also affect splicing itself (Graveley and Hertel, 2005). In turn, splicing affects EJC assembly and NMD. This is further complicated by the findings that some mRNAs of mammalian splicing factors, such as the SR-protein SRSF2, have alternative splicing products that are targeted by NMD (Lejeune and Maquat, 2005).

Understanding how the yeast SR-like proteins affect NMD might help to shed light on the intricate interplay of splicing, SR-proteins and NMD in higher eukaryotes and to unravel the basic NMD mechanisms. 


\section{Materials and methods}

\subsection{Materials}

Solutions and media that were prepared in the laboratory were sterilised by autoclaving at $121^{\circ} \mathrm{C}$ for $20 \mathrm{~min}$ or sterile filtration. Glassware was autoclaved, or sterilised at $180{ }^{\circ} \mathrm{C}$ for $5 \mathrm{~h}$.

Table 1 - List of consumable materials

\begin{tabular}{|c|c|}
\hline Materials & Manufacturer / Source \\
\hline Salmon sperm DNA & Sigma-Aldrich \\
\hline tRNAs & Sigma-Aldrich \\
\hline Oligonucleotides & Sigma-Aldrich \\
\hline Gibson Assembly® Master Mix & New England Biolabs \\
\hline qPCRBIO SyGreen Mix Lo-ROX & Nippon Genetics \\
\hline HDGreen $^{\mathrm{TM}}$ Plus DNA Stain & Intas Science Imaging \\
\hline GFP-Trap®_A beads & Chromotek \\
\hline GFP Selector beads & $\begin{array}{l}\text { NanoTag } \\
\text { Biotechnologies }\end{array}$ \\
\hline cOmplete ${ }^{\mathrm{TM}}$, EDTA-free Protease Inhibitor Cocktail & Roche \\
\hline Rotiphorese Gel 30 (37.5:1) acrylamide & Carl-Roth \\
\hline WesternBright ${ }^{\mathrm{TM}}$ Quantum ${ }^{\mathrm{TM}}$ Western Blotting HRP Substrate & Advansta \\
\hline $\begin{array}{l}\text { Amersham }{ }^{\mathrm{TM}} \mathrm{ECL}^{\mathrm{TM}} \text { Prime Western Blotting Detection } \\
\text { Reagent }\end{array}$ & GE Healthcare \\
\hline Amersham ${ }^{\mathrm{TM}}$ Protran ${ }^{\circledR} 0.45 \mathrm{NC}$ nitrocellulose membranes & GE Healthcare \\
\hline Whatman® Blotting Paper & Hahnemühle \\
\hline MF-Millipore ${ }^{\mathrm{TM}}$ Membrane Filter, $0.025 \mu \mathrm{m}$ pore size & Merck \\
\hline Poly-L-lysine solution & Sigma-Aldrich \\
\hline TRIzol TM Reagent & Thermo Fisher Scientific \\
\hline GlycoBlue $^{\mathrm{TM}}$ Coprecipitant & Thermo Fisher Scientific \\
\hline RiboLock RNAse inhibitor & Thermo Fisher Scientific \\
\hline \multicolumn{2}{|l|}{ Enzymes } \\
\hline Conventional Restriction Enzymes & Thermo Fisher Scientific \\
\hline FastAP Alkaline Phosphatase & Thermo Fisher Scientific \\
\hline KAPA HiFi DNA polymerase & Kapa Biosystems \\
\hline Phusion® High-Fidelity DNA polymerase & New England Biolabs \\
\hline Q5® High-Fidelity DNA polymerase & New England Biolabs \\
\hline DreamTaq DNA Polymerase & Thermo Fisher Scientific \\
\hline T4 DNA Ligase & Thermo Fisher Scientific \\
\hline RNase A & Qiagen \\
\hline RNase-Free DNase & Quiagen \\
\hline Zymolyase 20T & Zymo Research \\
\hline \multicolumn{2}{|l|}{ Protein and DNA markers } \\
\hline GeneRuler $^{\mathrm{TM}} 1 \mathrm{~kb}$ DNA Ladder & Thermo Fisher Scientific \\
\hline Lambda DNA/HindIII Marker & Thermo Fisher Scientific \\
\hline Lambda DNA/EcoRI plus HindIII Marker & Thermo Fisher Scientific \\
\hline PageRuler ${ }^{\mathrm{TM}}$ Prestained Protein Ladder & Thermo Fisher Scientific \\
\hline Cozy ${ }^{\mathrm{TM}}$ Prestained Protein Ladder & highQu \\
\hline
\end{tabular}


Table 1 - List of consumable materials (continued)

Materials

Kits

TURBO DNA-free ${ }^{\text {TM }}$ DNase Kit

NucleoSpin $\AA$ Gel and PCR Clean-up

NucleoSpin® Plasmid

NucleoSpin® RNA

NucleoBond(B Xtra Midi

FastGene ${ }^{\circledR}$ Scriptase II cDNA Kit

Maxima First Strand cDNA Synthesis Kit

\section{Manufacturer / Source}

Thermo Fisher Scientific MACHEREY-NAGEL MACHEREY-NAGEL MACHEREY-NAGEL MACHEREY-NAGEL NIPPON Genetics Thermo Fisher Scientific

Table 2 - List of equipment

\section{Equipment}

My Cycler 1.065

T100 ${ }^{\mathrm{TM}}$ Thermal Cycler

CFX Connect 96FX2 qPCR cycler

RotorGeneQ qPCR cycler

Heraeus $^{\mathrm{TM}} \mathrm{Pico}^{\mathrm{TM}} 21$

Heraeus $^{\mathrm{TM}}$ Fresco $^{\mathrm{TM}} 21$

Heraeus $^{\mathrm{TM}}$ Multifuge $^{\mathrm{TM}} \mathrm{X} 3$ with $\mathrm{TX}-750$ or $\mathrm{F} 15-8 \times 50$ cy rotor

Nano Drop 2000 spectrophotometer

Bio Photometer

AF6000 microscope with Leica DFC360 FX camera

Eclipse E400 tetrad microscope

Primo Star light microscope

Electro Blotter PerfectBlue Semi-Dry, Sedec M

INTAS UV gel detection system

Fusion-SL-3500.WL

Bio-Link 254 UV-crosslinking chamber

Improved Neubauer counting chamber

Milli-Q ${ }^{\circledR}$ Water purification system

FastPrep-24 ${ }^{\circledR}$ Cell homogenizer

Gene Pulser Xcell ${ }^{\mathrm{TM}}$ Electroporation System
Manufacturer / Source

Bio-Rad Laboratories

Bio-Rad Laboratories

Bio-Rad Laboratories

Qiagen

Thermo Fisher Scientific

Thermo Fisher Scientific

Thermo Fisher Scientific

Peqlab

Eppendorf

Leica

Nikon

Zeiss

Peqlab

INTAS

Vilber Lourmat

Vilber Lourmat

Carl Roth

Millipore

MP Biomedicals

Bio-Rad Laboratories 
Table 3 - List of software

\begin{tabular}{ll} 
Software & Source \\
\hline Rotor-Gene Q Software & Peqlab \\
\hline CFX manager 3.1 & Bio Rad \\
\hline Leica AF 2.7.3.9723 & Leica \\
\hline Fusion .Capt Software & Vilber Lourmat \\
\hline Bio1d & Vilber Lourmat \\
\hline Photoshop CS6 & Adobe \\
\hline Illustrator CS6 & Adobe \\
\hline Primer-BLAST & NCBI \\
\hline Snapgene & GSL Biotech \\
Office ${ }^{\circledR 2011 / 2019}$ & Microsoft
\end{tabular}

Table 4 - List of Escherichia coli media

LB

\begin{tabular}{|c|c|c|}
\hline \multicolumn{2}{|l|}{ Tryptone } & $1 \%(w / v)$ \\
\hline \multicolumn{2}{|l|}{ Yeast extract } & $0.5 \%(w / v)$ \\
\hline \multicolumn{2}{|l|}{$\mathrm{NaCl}$} & $85 \mathrm{mM}$ \\
\hline \multicolumn{2}{|c|}{ Ampicillin* (if added) } & $150 \mu \mathrm{g} / \mathrm{ml}$ \\
\hline \multicolumn{2}{|c|}{ Agar-Agar (for plates only) } & $1.5 \%(w / v)$ \\
\hline \multicolumn{3}{|l|}{$2 x Y T$} \\
\hline \multicolumn{2}{|l|}{ Tryptone } & $1.6 \%(w / v)$ \\
\hline \multicolumn{2}{|l|}{ Yeast extract } & $1 \%(w / v)$ \\
\hline \multicolumn{2}{|l|}{$\mathrm{NaCl}$} & $85 \mathrm{mM}$ \\
\hline \multicolumn{2}{|c|}{ Ampicillin* (if added) } & $150 \mu \mathrm{g} / \mathrm{ml}$ \\
\hline \multicolumn{2}{|l|}{ soc } & \\
\hline Yeast extract & $0.5 \%(w / v)$ & \\
\hline Peptone & $2 \%(w / v)$ & \\
\hline $\mathrm{NaCl}$ & $10 \mathrm{mM}$ & \\
\hline $\mathrm{KCl}$ & $2.5 \mathrm{mM}$ & \\
\hline $\mathrm{MgCl}_{2}$ & $10 \mathrm{mM}$ & \\
\hline $\mathrm{MgSO}_{4}$ & $10 \mathrm{mM}$ & \\
\hline Glucose & $20 \mathrm{mM}$ & \\
\hline
\end{tabular}

(Sambrook et al., 1989)

*Ampicillin was added after autoclaving and letting the medium cool down to $\sim 60^{\circ} \mathrm{C}$ or lower 
Table 5 - List of Saccharomyces cerevisiae media

YPD

\begin{tabular}{ll}
\hline Yeast extract & $1 \%(\mathrm{w} / \mathrm{v})$ \\
Peptone & $2 \%(\mathrm{w} / \mathrm{v})$ \\
Glucose & $2 \%(\mathrm{w} / \mathrm{v})$ \\
Agar-Agar & $1.8 \%(\mathrm{w} / \mathrm{v})$
\end{tabular}

Selective Media

\begin{tabular}{|c|c|}
\hline Nitrogen base & $1.7 \mathrm{~g} / \mathrm{l}$ \\
\hline Ammonium sulphate & $40 \mathrm{mM}$ \\
\hline Glucose* & $2 \%(w / v)$ \\
\hline Agar-Agar* & $1.8 \%(w / v)$ \\
\hline L-Alanine & 80 mg/l \\
\hline L-Arginine & 80 mg/l \\
\hline L-Asparagine & 80 mg/l \\
\hline L-Aspartic acid & 80 mg/l \\
\hline L-Cysteine & 80 mg/l \\
\hline L-Glutamine & $80 \mathrm{mg} / \mathrm{l}$ \\
\hline L-Glutamic acid & 80 mg/l \\
\hline L-Glycine & 80 mg/l \\
\hline Inositol & 80 mg/l \\
\hline L-Isoleucine & 80 mg/l \\
\hline L-Methionine & 80 mg/l \\
\hline Para- aminobenzoic acid & 8 mg/l \\
\hline L-Phenylalanine & 80 mg/l \\
\hline L-Proline & 80 mg/l \\
\hline L-Serine & $80 \mathrm{mg} / \mathrm{l}$ \\
\hline L-Threonine & 80 mg/l \\
\hline L-Tyrosine & 80 mg/l \\
\hline L-Valine & $80 \mathrm{mg} / \mathrm{l}$ \\
\hline \multicolumn{2}{|c|}{ optional metabolites according to selectivity } \\
\hline L-Adenine & 20 mg/l \\
\hline L-Histidine & 80 mg/l \\
\hline L-Leucine & $400 \mathrm{mg} / \mathrm{l}$ \\
\hline L-Lysine & 80 mg/l \\
\hline L-Tryptophan & $80 \mathrm{mg} / \mathrm{l}$ \\
\hline Uracil & 80 mg/l \\
\hline
\end{tabular}

(Sherman, 2002; Sprague, 1991; modified)

*components were autoclaved separately

${ }^{* *}$ components were sterile filtered

\section{Sporulation medium}

\begin{tabular}{|c|c|}
\hline Yeast extract & $0.25 \%(w / v)$ \\
\hline Potassium acetate & $150 \mathrm{mM}$ \\
\hline Glucose $^{\star *}$ & $0.05 \%(w / v)$ \\
\hline Uracil** $^{\star *}$ & $40 \mathrm{mg} / \mathrm{l}$ \\
\hline Adenine $^{* *}$ & $40 \mathrm{mg} / \mathrm{l}$ \\
\hline Tyrosine $e^{\star *}$ & $40 \mathrm{mg} / \mathrm{l}$ \\
\hline Histidine ${ }^{\star *}$ & $20 \mathrm{mg} / \mathrm{l}$ \\
\hline Leucine ${ }^{* *}$ & $20 \mathrm{mg} / \mathrm{l}$ \\
\hline Lysine** & $20 \mathrm{mg} / \mathrm{l}$ \\
\hline Tryptophan** & $20 \mathrm{mg} / \mathrm{l}$ \\
\hline Methionine ${ }^{\star *}$ & $20 \mathrm{mg} / \mathrm{l}$ \\
\hline Arginine ${ }^{\star \star}$ & 20 mg/l \\
\hline Phenylalanine ${ }^{* *}$ & $100 \mathrm{mg} / \mathrm{l}$ \\
\hline Threonine $^{\star \star}$ & $350 \mathrm{mg} / \mathrm{l}$ \\
\hline \multicolumn{2}{|l|}{ B-plates } \\
\hline Nitrogen Base & $0.17 \%(w / v)$ \\
\hline $\begin{array}{l}\text { Ammonium } \\
\text { sulphate }\end{array}$ & $3 \mathrm{mM}$ \\
\hline Agar-Agar* & $3 \%(w / v)$ \\
\hline Glucose* & $2 \%$ \\
\hline
\end{tabular}


Table 6 - List of S. cerevisiae strains

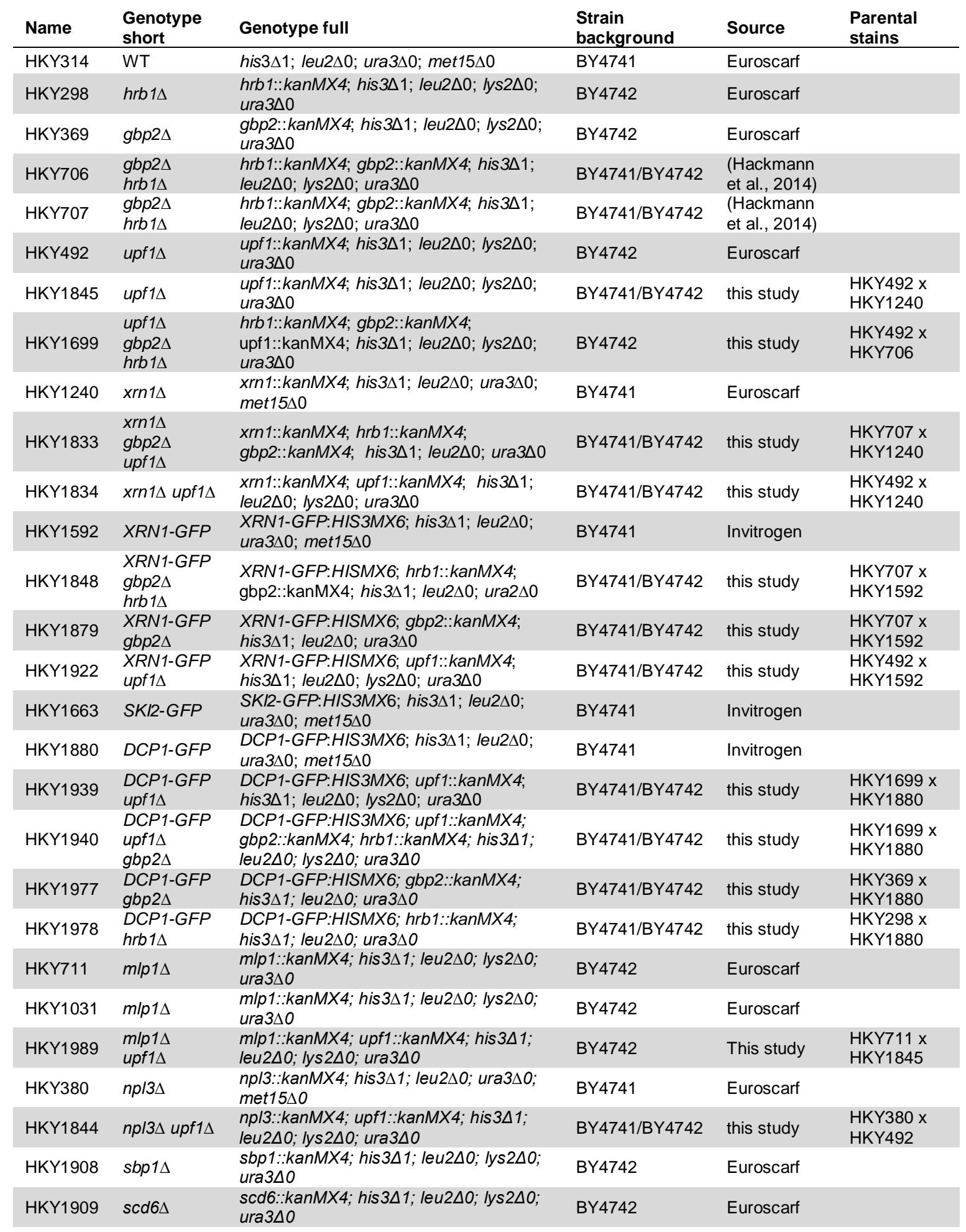


Table 7 - List of plasmids

\begin{tabular}{|c|c|c|}
\hline Name & Genotype & Source \\
\hline pHK12 & ${ }_{A D H} N L S-N E S-G F P-G F P ;$ URA3; CEN; $A M P^{R}$ & (Taura et al., 1998) \\
\hline pHK87 & LEU2, CEN, AMPR & (Sikorski and Hieter, 1989) \\
\hline pHK88 & URA3; CEN; AMPR & (Sikorski and Hieter, 1989) \\
\hline pHK367 & GBP2-GFP, URA3, CEN; AMPR & $\begin{array}{l}\text { (Windgassen and Krebber, } \\
\text { 2003) }\end{array}$ \\
\hline pHK385 & GAL1GBP2-GFP; URA3; CEN; $A M P^{R}$ & $\begin{array}{l}\text { (Windgassen and Krebber, } \\
\text { 2003) }\end{array}$ \\
\hline pHK1241 & GBP2-GFP; $2 \mu ; L E U 2 ; A M P^{R}$ & $\begin{array}{l}\text { Laboratory of Heike } \\
\text { Krebber }\end{array}$ \\
\hline pHK1333 & $\begin{array}{l}\text { GAL1GBP2-(S13/15/17A)-GFP; TRP1; } 2 \mu ; \\
A M P^{R}\end{array}$ & $\begin{array}{l}\text { Laboratory of Heike } \\
\text { Krebber }\end{array}$ \\
\hline pHK1225 & GBP2-(6x)MYC; CEN; URA3; AMPR & $\begin{array}{l}\text { Laboratory of Heike } \\
\text { Krebber }\end{array}$ \\
\hline pHK1244 & HRB1-GFP; LEU2; $2 \mu ; A M P^{R}$ & (Hackmann et al., 2014) \\
\hline pHK537 & HRB1-GFP; CEN; URA3; AMPR & (Häcker and Krebber, 2004) \\
\hline pHK1643 & HSP104-mRFP; URA3; CEN; AMPR & (Lee et al., 2010) \\
\hline pHK1649 & UPF1-HA; LEU2, CEN, AMPR & (Serdar et al., 2016) \\
\hline pHK1574 & CBP80-MYC; URA3; CEN; AMPR & $\begin{array}{l}\text { Laboratory of Heike } \\
\text { Krebber }\end{array}$ \\
\hline pHK1578 & CBP80 ${ }^{P T C}-M Y C ;$ URA3; CEN; AMPR & This study \\
\hline pHK1600 & GAL1MYC-CBP80 ${ }^{P T C} ;$ URA3; CEN; AMPR & This study \\
\hline pHK1642 & GAL1MYC-CBP80 ${ }^{P T C} ;$ HIS3; CEN; AMPR & $\begin{array}{l}\text { Laboratory of Heike } \\
\text { Krebber }\end{array}$ \\
\hline pHK1312 & 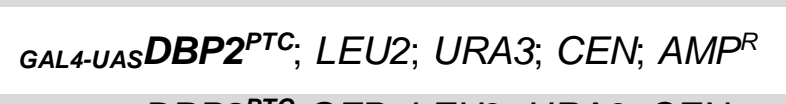 & $\begin{array}{l}\text { Laboratory of Heike } \\
\text { Krebber }\end{array}$ \\
\hline pHK1551 & $\begin{array}{l}\text { GAL4-UASDBP2 }{ }^{\text {PTC }} \text {-GFP; LEU2; URA3; CEN; } \\
\text { AMP }^{R}\end{array}$ & This study \\
\hline pHK1570 & $\begin{array}{l}\text { GAL4-UASDBP2-GFP; LEU2; URA3; CEN; } \\
\text { AMP }^{R}\end{array}$ & This study \\
\hline pHK1577 & $\begin{array}{l}\text { GAL4-UASMYC-DBP2 }^{\text {PTC; }} \text {; LEU2; URA3; CEN; } \\
\text { AMP }^{R}\end{array}$ & This study \\
\hline pHK1599 & 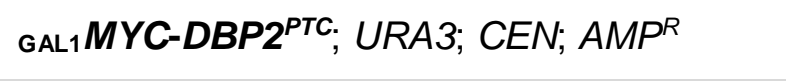 & This study \\
\hline pHK657 & GAL1 PGK1 $^{\text {PTC. }}$ LEU2; URA3; CEN; AMPR & (Muhlrad and Parker, 1994) \\
\hline pHK1639 & GAL1MYC-PGK1 $^{\text {PTC. }}$ URA3; CEN; AMPR & This study \\
\hline pHK1571 & ${ }_{A D H} M Y C-M T R 10 ; L E U 2, C E N, A M P^{R}$ & $\begin{array}{l}\text { Laboratory of Heike } \\
\text { Krebber }\end{array}$ \\
\hline
\end{tabular}


Table 8 - List of primers for qPCR

\begin{tabular}{|c|c|c|c|}
\hline Target & Name & Sequence & Remarks \\
\hline \multirow[t]{7}{*}{$\begin{array}{l}\text { MYC- } \\
\text { CBP80 }\end{array}$} & HK2782 & 5'-ACTTGAACGGATCATCCAGAGTCG-3' & $M Y C$ forward \\
\hline & HK2783 & 5'-AGGTGGGATTCTCTGTCATTTAGG-3' & CBP80 ORF reverse \\
\hline & HK3152 & 5'-AAGCTTCCTTTCGGGCTTTG-3' & NUF2 3' UTR reverse \\
\hline & HK3153 & 5'-CGCTATTCCACACGAATCCAC-3' & CBP80 ORF forward \\
\hline & HK3154 & 5'-GCGGAGTGATAACGAATGTAGTC-3' & CBP80 3' UTR reverse \\
\hline & HK3297 & 5'-TCCACACGAATCCACTAGGAG-3' & CBP80 ORF forward \\
\hline & HK3299 & 5'-ACGAATGTAGTCCATCCTCCG-3' & CBP80 3' UTR revers \\
\hline \multirow{2}{*}{$\begin{array}{l}\text { CBP80- } \\
\text { MYC }\end{array}$} & HK2696 & 5'-CGCTATTCCACACGAATCCA-3' & CBP80 ORF forward \\
\hline & HK2697 & 5'-ACTCTGGATGATCCGTTCAAGTC-3' & $M Y C$ reverse \\
\hline \multirow[t]{2}{*}{ GFP } & HK2134 & 5'-ATGCCCGAAGGTTATGTACAGG-3' & \\
\hline & HK2135 & 5'-CATTCTTTTGTTTGTCTGCCATG-3' & \\
\hline \multirow[t]{2}{*}{$\begin{array}{l}\text { MYC- } \\
\text { DBP2 }\end{array}$} & HK2701 & 5'-GAACGGATCATCCAGAGTCG-3' & MYC forward \\
\hline & HK2702 & 5'-CGGTAGTTACCGCCTTGTGG-3' & $D B P 2$ ORF reverse \\
\hline \multirow[t]{2}{*}{ RPL21B } & HK2652 & 5'-CGTTACCAAGTCTTCTGTTGG-3' & \\
\hline & HK2653 & 5'-GAGGAACGTTACCTTCAGTAG-3' & \\
\hline \multirow[t]{2}{*}{ RPL23B } & HK2319 & 5'-GTAAGCCAGAATTGAGAAAGAAGG-3' & \\
\hline & HK2320 & 5'-ATGGCAGAACCCTTCATTTCAC-3' & \\
\hline \multirow[t]{2}{*}{ ZWF1 } & HK3060 & 5'-GGTGAAGCCGATGACTCTAAGG-3' & \\
\hline & HK3061 & 5'-GGCCAGATAGAAGAGACGGTG-3' & \\
\hline \multirow[t]{2}{*}{ ADH1 } & HK3052 & 5'-TCAAGCCGCTCACATTCCTC-3' & \\
\hline & HK3053 & 5'-GCAGCACCGGAGATAGCAAC-3' & \\
\hline \multirow[t]{2}{*}{ EFB1 } & HK3046 & 5'-AAGGCTGAAAGAATTGCCGC-3' & \\
\hline & HK3047 & 5'-TCGATGGCCTTGACGTTAGC-3' & \\
\hline \multirow[t]{2}{*}{ ACT1 } & HK3048 & 5'-CGTCGGTAGACCAAGACACC-3' & \\
\hline & HK3049 & 5'-CCAGTTGGTGACAATACCGTG-3' & \\
\hline \multirow[t]{2}{*}{ Hem15 } & HK3058 & 5'-AAGATGGCGTGAAGAAGGCAG-3' & \\
\hline & HK3059 & 5'-GGCCAACGATCAATAACCGAC-3' & \\
\hline \multirow[t]{2}{*}{ 21s rRNA } & HK3089 & 5'-AGTTACGCTAGGGATAACAGGG-3' & mitochondrial \\
\hline & HK3090 & 5'-TGACGAACAGTCAAACCCTTC-3' & mitochondrial \\
\hline \multirow[t]{2}{*}{ RPS6A } & HK843 & 5'-CGTCATCTTCCTTGGACAAACC-3' & \\
\hline & HK877 & 5'-AAGGGTGAGCAAGAATTGGAAGG-3' & \\
\hline \multirow[t]{2}{*}{ RPL23A } & HK839 & 5'-GGTATCGAATCCAAGCAACC-3' & \\
\hline & HK840 & 5'-GCTTTTCCTTCTTTTTCTTTCCAC-3' & \\
\hline
\end{tabular}


Table 9 - List of primers for cloning

\begin{tabular}{|c|c|c|c|c|c|}
\hline $\begin{array}{l}\text { Cloned } \\
\text { construct }\end{array}$ & Name & Sequence & $\begin{array}{l}\text { Anneals } \\
\text { at }\end{array}$ & Orientation & $\begin{array}{l}\text { Underlined } \\
\text { feature }\end{array}$ \\
\hline \multirow[t]{2}{*}{ phk1578 } & HK2625 & $\begin{array}{l}\text { 5'- } \\
\text { GCCTAAAIGACAGAGAATCCCACCTGTTGTAC- } \\
3^{\prime}\end{array}$ & CBP80 & forward & Mismatch \\
\hline & HK2626 & 5'-GATTCTCTGTCA-ATTTAGGCATGCGAGGCC-3' & CBP80 & reverse & Mismatch \\
\hline \multirow[t]{6}{*}{ pHK1577 } & HK2576 & 5'-CCGAACAATAAAGATTCTAC-3' & $\begin{array}{l}\text { GAL4 } \\
\text { UAS }\end{array}$ & forward & \multirow{6}{*}{$\begin{array}{l}M Y C- \\
\text { sequence } \\
D B P 2 \\
\text { Promotor } \\
\text { sequence } \\
D B P 2 \\
\text { sequence } \\
\text { MYC } \\
\text { sequence }\end{array}$} \\
\hline & HK2577 & $\begin{array}{l}\text { 5'-GAAATCAACTTTTGTTCCATATTTGCTCTAAA- } \\
\text { TTTGCC-3' }\end{array}$ & $\begin{array}{l}\text { DBP2 } \\
\text { promoter }\end{array}$ & reverse & \\
\hline & HK2578 & $\begin{array}{l}\text { 5'- } \\
\text { AAGGCAAATTTAGAGCAAATATGGAACAAAAGT- } \\
\text { TGATTTCTG-3' }\end{array}$ & MYC & forward & \\
\hline & HK2579 & $\begin{array}{l}\text { 5'-TGATCTCTACCACCGTAAGTGTCCAGGACTC- } \\
\text { TGGATGATC-3' }\end{array}$ & MYC & reverse & \\
\hline & HK2580 & $\begin{array}{l}\text { 5'-GATCATCCAGAGTCGTCGACACTTACGGTG- } \\
\text { GTAGAGATC-3' }\end{array}$ & $D B P 2$ & forward & \\
\hline & HK2581 & 5'-GGTCTGTCATTGTAAGAGTTTC-3' & $D B P 2$ & reverse & \\
\hline \multirow[t]{4}{*}{ pHK1551 } & HK2159 & $\begin{array}{l}\text { 5'-CAGAAATCATTTACTTTGTTTTCCTTTTTTTTA- } \\
\text { AGT-3' }\end{array}$ & $D B P 2$ & forward & \multirow{4}{*}{$\begin{array}{l}\text { GFP } \\
\text { sequence } \\
\text { GFP } \\
\text { sequence }\end{array}$} \\
\hline & HK2161 & $\begin{array}{l}\text { 5'-GAGTTCTTCTCCTTTGCTAGCCATAGC- } \\
\text { TCGACAATAGTTTGAACGACCTC-3' }\end{array}$ & $D B P 2$ & reverse & \\
\hline & HK2162 & $\begin{array}{l}\text { 5'-CTGGGATTACACATGGCATGGATGAAC- } \\
\text { IATACAAAGGATCAGTCAAAGTGATTC-3' }\end{array}$ & $\begin{array}{l}D B P 2 \\
\text { terminator }\end{array}$ & forward & \\
\hline & HK6263 & $\begin{array}{l}\text { 5'-GATCCTCTAGAGTCGACCTGCAGGCATGC- } \\
\text { AAGCTTG-3' }\end{array}$ & $\begin{array}{l}\text { pHK1312 } \\
\text { backbone }\end{array}$ & reverse & \\
\hline \multirow[t]{2}{*}{ pHK1570 } & HK2222 & 5'-TCGGAGCTCGTTGAGAATTCGAAATTCC-3' & $\begin{array}{l}D B P 2 \\
\text { promoter }\end{array}$ & forward & \multirow[t]{2}{*}{ Sac1 site } \\
\hline & HK2021 & 5'-CCACCATAAGATCTCCTGTC-3' & $D B P 2$ & reverse & \\
\hline \multirow[t]{2}{*}{ pHK1599 } & HK2742 & $\begin{array}{l}\text { 5'-TACTTTAACGTCAAGGAGAAAAAACTATAGC- } \\
\text { ATGCATGGAACAAAAGTTGATTTCTG-3' }\end{array}$ & MYC & forward & \multirow{2}{*}{$\begin{array}{l}\text { GAL1 } \\
\text { promotor } \\
\text { sequence } \\
\text { pHK385 } \\
\text { Backbone } \\
\text { sequence }\end{array}$} \\
\hline & HK2743 & $\begin{array}{l}\text { 5'-TTAACCCTCACTAAAGGGAACAAAAGCTG- } \\
\text { GGTACCGGGTTGTACGAGAACAAAG-3' }\end{array}$ & $\begin{array}{l}D B P 2 \\
\text { terminator }\end{array}$ & reverse & \\
\hline \multirow[t]{4}{*}{ pHK1600 } & HK2770 & $\begin{array}{l}\text { 5'-TACTTTAACGTCAAGGAGAAAAAACTATAG- } \\
\text { CATGCATGAACAAAAGTTGATTTCTGAAG-3' }\end{array}$ & MYC & forward & $\begin{array}{l}\text { GAL1 } \\
\text { promotor } \\
\text { sequence }\end{array}$ \\
\hline & HK2771 & 5'gtcgacGACTCTGGATGATC-3' & MYC & reverse & \\
\hline & HK2772 & $\begin{array}{l}\text { 5'-AAGAAGACTTGAACGGATCATCCAGAGTC- } \\
\text { GTCGACTTTAATAGAAAAAGAAGAGGAGG-3' }\end{array}$ & CBP80 & forward & \multirow{2}{*}{$\begin{array}{l}\text { MYC } \\
\text { sequence } \\
\text { pHK385 } \\
\text { Backbone } \\
\text { sequence }\end{array}$} \\
\hline & HK2773 & $\begin{array}{l}\text { 5'-AAGCTTCCTTTCGGGCTTTGTTAGCAGCC- } \\
\text { GGATCCAACTTCCTTTGTTTCTTGAATC-3' }\end{array}$ & CBP80 & reverse & \\
\hline \multirow[t]{2}{*}{ pHK1639 } & HK3091 & $\begin{array}{l}\text { 5'-TCTCAGAAGAAGACTTGAACGGATCATC- } \\
\text { CAGAGTCGTCGACATGTCTTTATCTTCAAA- } \\
\text { GTTGTC-3' }\end{array}$ & $P G K 1$ & forward & $\begin{array}{l}\text { MYC } \\
\text { sequence }\end{array}$ \\
\hline & HK3092 & $\begin{array}{l}\text { 5'-TCGGAATTAACCCTCACTAAAGGGAACAAAA- } \\
\text { GCTGGGTACCAAGCTTTAACGAACGCAGAAT-3' }\end{array}$ & $\begin{array}{l}\text { PGK1 } \\
\text { terminator }\end{array}$ & reverse & $\begin{array}{l}\text { pHK385 } \\
\text { Backbone } \\
\text { sequence }\end{array}$ \\
\hline \multirow[t]{2}{*}{ pHK1667 } & HK3340 & $\begin{array}{l}\text { 5'-AGGGCGAATTGGAGCTCCACCGCGGTG- } \\
\text { GCGGCCGCTCTAGACAGAGCGGAAGCTC-AGC- } \\
\text { 3' }\end{array}$ & $\begin{array}{l}C D C 33 \\
\text { promoter }\end{array}$ & forward & $\begin{array}{l}\text { pHK12 } \\
\text { Backbone } \\
\text { sequence }\end{array}$ \\
\hline & HK3341 & $\begin{array}{l}\text { 5'-CAACTCCAGTGAAGAGTTCTTCTCCTTTG- } \\
\text { CTAGCCATAGCTCGAGCCAAGGTGATTGAT- } \\
\text { GGTTGAGG-3' }\end{array}$ & CDC33 & reverse & $\begin{array}{l}\text { GFP } \\
\text { sequence }\end{array}$ \\
\hline
\end{tabular}




\subsection{DNA cloning}

\subsubsection{Amplification of DNA by PCR}

For analytical purposes, DNA fragments were amplified using the Dream Taq polymerase. Preparatory amplifications of DNA, to be use for cloning, were performed using a proofreading polymerase (see Table 10). As the DNA template, either 5-100 ng plasmid DNA or $1 \mu$ l of yeast genomic DNA (see 3.2.5) was used. The reaction mix was incubated in a thermal cycler (incubation times and temperatures see Table 11). The reaction mixes and cycling conditions are based on the protocols supplied with the polymerases. The PCR products were subsequently analysed using agarose gel electrophoresis (see 3.2.3).

Table 10 - PCR reaction mix composition

\begin{tabular}{lllll} 
Polymerase: & Dream Taq & Phusion & KAPAHiFi & Q5 \\
\hline dNTPs & $200 \mu \mathrm{M}$ each & $200 \mu \mathrm{M}$ each & $300 \mu \mathrm{M}$ each & $200 \mu \mathrm{M}$ each \\
Primers & $0.2 \mu \mathrm{M}$ each & $0.5 \mu \mathrm{M}$ each & $0.3 \mu \mathrm{M}$ each & $0.5 \mu \mathrm{M}$ each \\
Polymerase & $0.025 \mathrm{U} / \mu \mathrm{l}$ & $0.02 \mathrm{U} / \mu \mathrm{l}$ & $0.02 \mathrm{U} / \mu \mathrm{l}$ & $0.02 \mathrm{U} / \mu \mathrm{l}$
\end{tabular}

Table 11 - PCR incubation temperatures and times

\begin{tabular}{lllll} 
Polymerase: & Dream Taq & Phusion & KAPA HiFi & Q5 \\
\hline $\begin{array}{l}\text { Initial } \\
\text { denaturation }\end{array}$ & $95^{\circ} \mathrm{C}-3 \mathrm{~min}$ & $98^{\circ} \mathrm{C}-30 \mathrm{~s}$ & $95^{\circ} \mathrm{C}-5 \mathrm{~min}$ & $98^{\circ} \mathrm{C}-30 \mathrm{~s}$ \\
\hline Denaturation & $95^{\circ} \mathrm{C}-30 \mathrm{~s}$ & $98^{\circ} \mathrm{C}-10 \mathrm{~s}$ & $98^{\circ} \mathrm{C}-20 \mathrm{~s}$ & $98^{\circ} \mathrm{C}-10 \mathrm{~s}$ \\
Annealing & $45-60^{\circ} \mathrm{C}$ & $45-60^{\circ} \mathrm{C}$ & $45-60^{\circ} \mathrm{C}$ & $45-60{ }^{\circ} \mathrm{C}$ \\
& $-30 \mathrm{~s}$ & $-30 \mathrm{~s}$ & $-15 \mathrm{~s}$ & $-30 \mathrm{~s}$ \\
\hline Extension & $72{ }^{\circ} \mathrm{C}-1 \mathrm{~min} / \mathrm{kb}$ & $72{ }^{\circ} \mathrm{C}-30 \mathrm{~s} / \mathrm{kb}$ & $72{ }^{\circ} \mathrm{C}-30 \mathrm{~s} / \mathrm{kb}$ & $72^{\circ} \mathrm{C}-30 \mathrm{~s} / \mathrm{kb}$ \\
\hline
\end{tabular}

35 cycles (for analytical purpose) or 30 cycles (for preparatory purpose)

Final extension $\quad 72^{\circ} \mathrm{C}-10 \min \quad 72^{\circ} \mathrm{C}-10 \min \quad 72^{\circ} \mathrm{C}-5 \min \quad 72^{\circ} \mathrm{C}-10 \min$




\subsubsection{Cleavage of DNA by restriction digestion}

To cleave DNA, samples were digested with standard restriction endonucleases (Thermo Fisher Scientific). For the digestion, 0.5 or $3 \mu \mathrm{g}$ of plasmid DNA (for analytical and preparatory purposes, respectively) or a purified PCR product was mixed with $5 \mathrm{U}$ of the desired restriction enzyme in one of the provided buffers (see Table 12). Plasmid DNA was digested in a total volume of $20 \mu \mathrm{l}$ and PCR products in $30 \mu \mathrm{l}$. The samples were incubated at $37^{\circ} \mathrm{C}$ for $4-15 \mathrm{~h}$. For cloning purposes, the $5^{\prime}$ and 3 ' phosphate groups of plasmids were removed after restriction digestion to prevent self-ligation. Directly after the enzymatic cleavage, $1 \mathrm{U}$ od FastAP alkaline phosphatase (Thermo Fisher Scientific) was added to the reaction mix and incubated for $10 \mathrm{~min}$ at $37^{\circ} \mathrm{C}$. Cleaved DNA fragments were visualised by agarose gel electrophoresis

Table 12 - Buffers for double restriction digests

\begin{tabular}{lll}
\multicolumn{2}{l}{ Restriction enzymes } & Buffer \\
\hline EcoRI & BamHI & 2x Tango \\
Xhol & BamHI & 2x Tango \\
BgllI & BamHI & 2x Tango \\
Sacl & Bglll & 2x Tango \\
Sacl & Sacll & 1x Tango \\
Bglll & HindlII & 2x Tango \\
EcoRI & Kpnl & BamHI buffer \\
Sacl & Kpnl & BamHI buffer \\
Xhol & Xbal & 2x Tango \\
\hline
\end{tabular}

\subsubsection{Agarose gel electrophoresis}

DNA samples were separated by size using agarose gel electrophoresis. For casting gels, $1 \%(\mathrm{w} / \mathrm{v})$ agarose was added to TAE buffer (40 mM Tris base, $0.1 \%(\mathrm{v} / \mathrm{v})$ Acetic acid, $1 \mathrm{mM}$ EDTA) and heated in a standard microwave until the agarose was completely dissolved. After letting the mixture cool down to approx. $60^{\circ} \mathrm{C}$ under constant stirring, either $0.3 \mu \mathrm{g} / \mathrm{ml}$ ethidium bromide or $5 \mu \mathrm{l} / 100 \mathrm{ml}$ HDGreen Plus DNA Stain (Intas Science Imaging) was added. After further stirring, the agarose solution was cast into a gel mould (approx. $10 \mathrm{~cm}$ $\times 15 \mathrm{~cm} \times 2 \mathrm{~cm}$ ) and a comb was inserted immediately to form sample wells. After letting the gel cool down to room temperature, it was used directly or stored at $4{ }^{\circ} \mathrm{C}$.

For the electrophoretic separation of DNA samples, the agarose gel was placed in a gel chamber filled with sufficient TAE buffer to cover the gel. The DNA samples were mixed with a $6 x$ loading dye $(10 \mathrm{mM}$ Tris $\mathrm{pH}$ 7.6, $60 \mathrm{mM}$ EDTA, $60 \%$ (v/v) glycerol, $0.03 \%$ (w/v) bromophenol blue) and pipetted into the sample wells. A voltage of $120 \mathrm{~V}$ was applied to the electrodes of the gel chamber. After $30-90$ min the DNA bands were visualised on a UV-transilluminator at $320 \mathrm{~nm}$. 


\subsubsection{DNA extraction from agarose gels and enzymatic reactions}

All DNA fragments were purified with the silica membrane based NucleoSpin Gel and PCR Clean-up kit (MACHEREY-NAGEL), following the manufacturer's instructions. DNA was eluted in $5 \mathrm{mM}$ Tris/ $\mathrm{HCl}$, pHK8.5.

\subsubsection{Extraction of genomic DNA from S. cerevisiae cells}

Yeast DNA was extracted by phenol/chloroform extraction based on Rose et al., 1990. Yeast cells were grown in $10 \mathrm{ml}$ YPD medium until saturation. The culture was harvested by centrifugation at $2500 \mathrm{x} \mathrm{g}$ for $5 \mathrm{~min}$. The supernatant was removed; the pellet was washed with $1 \mathrm{ml}$ water and transferred into a $2 \mathrm{ml}$ screw top tube. For the lysis, $500 \mu \mathrm{l}$ detergent lysis buffer (2 \% Triton X-100, 1 \% SDS, $100 \mathrm{mM} \mathrm{NaCl}, 1 \mathrm{mM}$ EDTA, $10 \mathrm{mM}$ Tris pH 8), $500 \mu \mathrm{l}$ phenol and $300 \mu \mathrm{l}$ glass beads $(0.4-0.6 \mathrm{~mm})$ were added and the cells were homogenised twice at $4 \mathrm{~m} / \mathrm{s}$ for $30 \mathrm{~s}$. The samples were centrifuged at $16000 \mathrm{xg}$ for $5 \mathrm{~min}$ to separate the organic and the aquatic phase. The hydrophilic DNA molecules remain in the upper, aquatic phase. The aquatic phase was transferred into a new tube, mixed with an equal volume of phenol and the phases were separated again by centrifugation. The aquatic phase was mixed in a new tube with an equal volume of phenol chloroform isoamyl alcohol (25:24:1) and centrifuged again. The last step was repeated once in the same manner and once with an equal volume of chloroform isoamyl alcohol (24:1). The remaining aquatic phase was transferred again into a new tube and mixed with $0.1 \mathrm{x}$ volume $3 \mathrm{M}$ sodium acetate $(\mathrm{pH} \mathrm{5.2)}$ and $3 x$ volume $100 \%$ ethanol. After mixing, the sample was incubated approx. $12 \mathrm{~h}$ at $-20{ }^{\circ} \mathrm{C}$. Precipitated DNA was pelleted by centrifugation at 16000x $\mathrm{g}$ for $30 \mathrm{~min}$ at $4{ }^{\circ} \mathrm{C}$. The DNA pellet was washed twice with $70 \%$ ethanol and dried for approx. $5 \mathrm{~min}$ at $65{ }^{\circ} \mathrm{C}$. To resolve the DNA, $100 \mu \mathrm{l}$ water was added and incubated at $65{ }^{\circ} \mathrm{C}$ for 10 minutes with shaking.

To use the genomic DNA as template for PCR, it was first digested by a restriction enzyme that does not cut in the desired sequence. For this, $1 \mu$ prepared genomic DNA was digested in $20 \mu \mathrm{l}$ (see 3.2.2) for approx. $15 \mathrm{~h}$, followed by heat inactivation according to the enzyme's specifications from the manufacturer. After the digestion, $1 \mu \mathrm{l}$ was used for one PCR reaction (see 3.2.1). The DNA concentration is dependent the yield of the DNA preparation, however, as RNA is not removed in this protocol, the DNA concentration cannot be measured. 


\subsubsection{Measurement of DNA and RNA concentrations}

Concentrations of nucleic acids were determined by the absorbance of light at $260 \mathrm{~nm}$ wavelength, according to the Lambert-Beer law, using a NanoDrop spectrophotometer. In all cases, a baseline correction at $340 \mathrm{~nm}$ and extinction coefficients of $50 \mathrm{ng} \times \mathrm{cm} / \mu \mathrm{l}$ for double stranded DNA and $40 \mathrm{ng} \times \mathrm{cm} / \mu \mathrm{l}$ for RNA were used.

\subsubsection{Ligation of DNA}

To ligate DNA fragments, 100 - $150 \mathrm{ng}$ of the vector fragment was mixed with the $2-3$-fold molar amount of insert fragment. This was mixed with T4 ligase (Thermo Fisher Scientific) in the provided buffer (see Table 13). In all cases sticky ends were ligated, either at $21^{\circ} \mathrm{C}$ for $4 \mathrm{~h}$ or at $16{ }^{\circ} \mathrm{C}$ for approx. $15 \mathrm{~h}$.

Table 13 - composition of DNA ligation mix

\begin{tabular}{ll} 
Vector & $100-150 \mathrm{ng}$ \\
\hline Insert & 2 - 3-fold molar excess \\
\hline 10x T4 DNA ligase Buffer & $1 \mu \mathrm{l}$ \\
T4 ligase & $5 \mathrm{U}$ \\
Total reaction volume & $10 \mu \mathrm{l}$
\end{tabular}

\subsubsection{Gibson Assembly}

For the Gibson assembly of DNA fragments, $100-150 \mathrm{ng}$ of the vector fragment was mixed with the 2 - 3-fold molar amount of insert fragment (5-fold molar excess for insert fragments shorter than $200 \mathrm{bp}$ ) in a total volume of $10 \mu \mathrm{l}$. After addition of $10 \mu \mathrm{l} 2 x$ Gibson Assembly Master Mix (New England Biolabs), the samples were incubated at $50{ }^{\circ} \mathrm{C}$ for $1 \mathrm{~h}$ and subsequently used for transformation of E. coli. For the Gibson assembly, all fragments were designed to have $30-40$ bp overlap by adding the respective sequence to the primers used for generating the insert fragments. A 5' exonuclease in the provided master mix shortens the 5' ends and thereby generates 3' overhangs. Due to the added sequence overlap, the overhangs can anneal. The gaps are filled by a polymerase and a ligase forms the final phosphodiester bond (Gibson et al., 2009). 


\subsubsection{Transformation of $E$. coli with plasmid DNA}

\section{Heat shock transformation}

For all cloning purposes, the DH5a Escherichia coli strain was used. A $100 \mu$ suspension of chemically competent cells (prepared according to Inoue, Nojima, \& Okayama, 1990) was thawed on ice and mixed with $100 \mathrm{ng}$ vector or a complete ligation product (see 3.2.7) or Gibson Assembly product (see 3.2.8) and mixed gently. After 30 min of incubation on ice, the cells were exposed to a heat shock at $42{ }^{\circ} \mathrm{C}$ for $2 \mathrm{~min}$. The cells were immediately placed on ice and $1 \mathrm{ml}$ of SOC medium was added. After $20-45 \mathrm{~min}$ at $37^{\circ} \mathrm{C}$ the cells were centrifuged at $600 \times \mathrm{g}$ for $5 \mathrm{~min}$ and resuspended in approx. $100 \mu \mathrm{l}$ residual liquid. The suspension was plated on selective LB-plates and incubated at $37^{\circ} \mathrm{C}$ for $10-15 \mathrm{~h}$. As all used vectors contained an ampicillin resistance, E. coli were always selected with ampicillin.

\section{Electroporation}

Prior to the transformation, the DNA sample was pipetted on a nitrocellulose membrane (0.025 $\mu \mathrm{m}$ pore size), which was placed on approx. $25 \mathrm{ml}$ deionised water in a Petri dish. To remove salts, the sample was dialysed in this manner for approx. 60 min. A $50 \mu \mathrm{l}$ suspension of electro competent E. coli (prepared according to Dower et al., 1988) was thawed on ice and $10 \mu \mathrm{l}$ of the dialysed DNA sample was added and the suspension was gently mixed. The cell suspension was pipetted between the electrodes of an electroporation cuvette (1 mm gap), which was precooled on ice. With an electroporator, a pulse (exponential decay, $1500 \mathrm{~V}, 50 \mu \mathrm{F}, 150 \Omega$ ) was applied and $1 \mathrm{ml} \mathrm{SOC}$ medium was immediately added. After $20-45 \mathrm{~min}$ at $37^{\circ} \mathrm{C}$, the cells were centrifuged at $600 \times \mathrm{g}$ for $5 \mathrm{~min}$ and resuspended in approx. $100 \mu \mathrm{l}$ residual liquid. The suspension was plated on ampicillin containing LB-plates and incubated at $37^{\circ} \mathrm{C}$ for $10-15 \mathrm{~h}$.

\subsubsection{Plasmid purification from $E$. coli cultures}

Plasmids were purified using either the NucleoSpin Plasmid purification kit (Macherey Nagel, silica membrane based) for small scale purifications or the NucleoBond Xtra midi kit (Macherey Nagel, methyl-amino-ethanol based anion-exchanger) for larger preparations, following the manufacturer's instructions. E. Coli were grown in $10 \mathrm{ml}$ or $100 \mathrm{ml} 2 \mathrm{x}$ YT medium until saturation and DNA was purified according to the manufacturer's instructions. The yield of isolated plasmid DNA was determined by photometric analysis (see 3.2.6). 


\subsubsection{E. coli colony PCR}

To screen several E. coli colonies after transformation with a ligation or Gibson assembly product for the correct DNA construct, colonies were picked from the ampicillin-containing LB-plate with sterile toothpicks. The toothpick was inserted into an empty PCR tube to transfer some bacterial material and subsequently placed in $10 \mathrm{ml}$ ampicillin-containing 2x YT medium. A Dream Taq PCR reaction mix was added to the PCR tube and a PCR run was performed as described in 3.2.1. For a colony PCR, the initial denaturation time was increased to $15 \mathrm{~min}$ at $95^{\circ} \mathrm{C}$. The culture, which were inoculated with the toothpick, was incubated for approx. $15 \mathrm{~h}$ at $37^{\circ} \mathrm{C}$ on a rotator. Plasmids from selected colonies were isolated as described in 3.2.10.

\subsubsection{Site directed mutagenesis}

The point mutation in the CBP80 NMD reporters was introduced in a PCR based mutagenesis approach (Liu and Naismith, 2008). For this, primers were designed to contain the nonsense mutation. Both primers had an overlapping sequence of $19 \mathrm{bp}$. A PCR was performed with the proof-reading KAPAHifi polymerase (see 3.2.1). In this application the extension step continues around the entire plasmid backbone to form complete, new DNA strands of a plasmid that contain the mutated sequence. To destroy the parental DNA strands, $0.4 \mathrm{U} / \mu \mathrm{l}$ of Dpnl were added and incubated for approx. $15 \mathrm{~h}$ at $37^{\circ} \mathrm{C}$. Dpnl cleaves in N6-methyladenine containing 5'-GATC-3' sites, which are the methylation site of the Dam methylase. Plasmids purified from DH5a E. coli cells are Dam-methylated in the Dpnl restriction site, and thereby destroyed by the restriction enzyme. As Dpnl also cleaves hemimethylated DNA, hybrids of parental and mutated DNA strands are destroyed in the same manner. Due to the overlap of the two primers, a nicked plasmid can form. After Dpnl restriction digestion, the complete reaction product was used for transformation of $E$. coli cells (see 3.2.9).

\subsubsection{Sequencing of plasmid DNA}

In all cloned DNA constructs, the gene of interest was sequenced by LGC Genomics, using Sanger sequencing. 


\subsection{S. cerevisiae cell culture}

\subsubsection{General yeast cell culture conditions}

Yeast cells without plasmid DNA were grown in YPD medium (for yeast media see Table 5; page 35). All plasmid containing yeast cells were grown in selective media, based on uracil, histidine and/or leucine prototrophy (Sherman, 2002). The used BY4741 and BY4742 yeast strain backgrounds contain the ura3 $\Delta 0$, his $3 \Delta 0$ and leu2 $\Delta 0$ knockouts for this selection purpose and are therefore auxotrophic for the three substances. The employed plasmids contain a URA3, HIS3 or LEU2 marker gene to grant prototrophy for one of the three compounds.

The plasmids pHK1312, pHK1551, pHK1570 and pHK1577 contain both a URA3 and a LEU2 marker. Cells with one of these plasmids were grown in uracil selective medium.

For long-term storage, the yeast strains were kept in $50 \%$ glycerol at $-80{ }^{\circ} \mathrm{C}$. From these stocks, yeast cells were streaked out on YPD agar plates. Yeast strains on YPD or selective agar plates were grown for $2-5$ days and kept at $4{ }^{\circ} \mathrm{C}$. In regular intervals, the cells were re-streaked onto new agar plates or fresh cells were streaked out from the frozen stocks. Unless otherwise stated, yeast cells were always grown at $25^{\circ} \mathrm{C}$. For each experiment or for transformation with plasmid DNA, cell material from the agar plates was used to inoculate $5-20 \mathrm{ml}$ liquid cultures in culture tubes and grown in a rotator. For a lager yeast culture, a $5-20 \mathrm{ml}$ liquid pre-culture was grown for 1 day and used for inoculation of a $50-400 \mathrm{ml}$ culture, which was grown in an Erlenmeyer flask on a shaker (approx. 120 rpm). For all experiments, the yeast cultures were grown over night and harvested the next day before reaching the stationary growth phase at an $\mathrm{OD}_{600}$ of $0.8-1.3$ or $2 \times 10^{7}-3 \times 10^{7}$ cells/ml (see 3.3.2). In all experiments, where equal amounts of cell material were harvested, the volume of harvested cells was adjusted according to the measured cell density. Yeast cells were harvested by centrifugation in $50 \mathrm{ml}$ tubes or $400 \mathrm{ml}$ centrifuge beakers at 2000x g for $5 \mathrm{~min}$. The cell pellets were resuspended in $1 \mathrm{ml}$ of water, transferred into $1.5 \mathrm{ml}$ or $2 \mathrm{ml}$ tubes and centrifuged at $16000 \mathrm{x}$ for $30-60 \mathrm{~s}$. To wash the cells in a buffer as described in the respective protocol, the cell pellet was resuspended in $1 \mathrm{ml}$ buffer, followed by $30-60$ s centrifugation at $16000 x \mathrm{~g}$. Yeast cell pellets were either used directly or frozen in liquid nitrogen and kept at $-20^{\circ} \mathrm{C}$. 


\subsubsection{Measurement of yeast cell density in liquid cultures}

\section{Cell counting in a Neubauer counting chamber}

For Fluorescence microscopy, the cell density of yeast cell cultures was determined by cell counting in an improved Neubauer counting chamber. A sample of the liquid cell culture was diluted $1 / 40$ (for log phase cultures) or $1 / 100$ (for stationary cultures) and $10 \mu$ l were pipetted into the counting chamber. Before each pipetting step, the cell suspension was mixed to avoid errors due to cell sedimentation. On the grid of the counting chamber, the cells in $0.1 \mu \mathrm{l}$ were counted to extrapolate the number of cells per $\mathrm{ml}$.

\section{Measurement of the optical density}

Light scattering by yeast cells that are suspended in liquid medium causes a turbidity of the culture that is dependent on the cell density. The established measurement of the optical density at $600 \mathrm{~nm}$ wavelength $\left(\mathrm{OD}_{600}\right)$ was used to assess the cell density of liquid yeast cultures. For this, $1 \mathrm{ml}$ of the yeast culture (or a dilution of it) was pipetted into a $1 \mathrm{ml}$ plastic cuvette and the $\mathrm{OD}_{600}$ was measured in a standard photometer. A sample of the respective medium served as a blank value and was subtracted from all measured values to account for the optical density of the medium itself. The $\mathrm{OD}_{600}$ correlates linearly with the cell density in the range of $\mathrm{OD}_{600} 0.1-1 . \mathrm{An} \mathrm{OD}_{600}>0.8$ was measured by diluting the culture in the same medium and using the dilution factor to calculate a theoretical $\mathrm{OD}_{600}$ that follows a linear correlation. All stated $\mathrm{OD}_{600}$ values $>0.8$ were measured this way and therefore tend to be higher than the actual optical densities these cultures had. For cultures in YPD medium, all $\mathrm{OD}_{600}$ values $>0.5$ were measured in a dilution, due to the higher optical density of the medium itself.

\subsubsection{Transformation of yeast cells with plasmid DNA}

Plasmid DNA was introduced into Yeast cells by lithium acetate heat shock transformation (Gietz et al., 1992). A $5 \mathrm{ml}$ liquid pre-culture was grown to saturation and used to inoculate a $20 \mathrm{ml}$ liquid culture with a cell density of approx. $0.5 \times 10^{7} \mathrm{cell} / \mathrm{s} / \mathrm{ml}$. This culture was grown to $1-3 \times 10^{7}$ cells $/ \mathrm{ml}$ and cells were harvested. The cell pellet was washed once with water and once with TE lithium acetate buffer (10 mM Tris, $1 \mathrm{mM}$ EDTA, $100 \mathrm{mM}$ lithium acetate, $\mathrm{pH}$ 7). The pellet was resuspended in TE lithium acetate buffer. The volume of buffer was adjusted to generate a cell density of approx. $1 \times 10^{9} \mathrm{cells} / \mathrm{ml}$. Of this cell suspension, $50 \mu \mathrm{l}$ $\left(0.5 \times 10^{8}\right.$ cells $)$ were mixed with $1 \mu \mathrm{g}$ plasmid DNA, $50 \mu \mathrm{g}$ salmon sperm carrier DNA and $300 \mu$ I PEG TE lithium acetate buffer (10 mM Tris pH 7.5, 1 mM EDTA, 100 mM lithium acetate, $40 \%(\mathrm{v} / \mathrm{v})$ poly ethylene glycol 4000$)$. The carrier DNA was heated at $95{ }^{\circ} \mathrm{C}$ for 5 min and put on ice for $\sim 2$ min prior to adding it to the cell suspension. For transformation with two different plasmids in one process, $1 \mu \mathrm{g}$ of each plasmid was added. The sample 
was mixed and incubated at $25^{\circ} \mathrm{C}$ for $30 \mathrm{~min}$ on a rotator, followed by 15 min heat shock at $42{ }^{\circ} \mathrm{C}$. After the heat shock, the cells were centrifuged at $16000 \mathrm{x} \mathrm{g}$ for $1 \mathrm{~min}$ and the pellet was washed with $1 \mathrm{ml}$ of water. After a second centrifugation, the cell pellet was resuspended in $100 \mu \mathrm{l}$ of water and the cell suspension was plated on a selective plate according to the plasmids selective marker and grown for $2-3$ days.

\subsubsection{Crossing of yeast strains}

\section{Crossing}

All yeast strains, used in this study, are haploid strains. Two haploid strains of opposite mating types (MATa or MATa, as determined by the respective gene cassette in the MAT locus) can form diploids. Nutrient depletion causes the cells to sporulate. They undergo miosis, and one diploid forms 4 haploid cells in an ascus (Sherman, 2002; Sherman and Hicks, 1991).

To cross two different mutants, both strains were streaked out and mixed on a YPD plate. After 2 - 3 days of growth, diploids were selected, if possible, by the combination of marker genes. For this, cell material was streaked out on the respective selective plate and grown for $2-3$ days. To induce sporulation, cell material from the selective plate (or YPD plate if no diploid selection was possible) was used to inoculate $2 \mathrm{ml}$ of sporulation medium (see Table 5, page 35). After $5-8$ days at $25^{\circ} \mathrm{C}$ in a rotator, the presence of tetrads (asci with 4 spores) was verified using a light microscope and $100 \mu$ of the cell culture were centrifuged at $16000 \mathrm{xg}$ for $1 \mathrm{~min}$, washed once with a $\mathrm{ml}$ of water and resuspended in $50 \mu \mathrm{l}$ P-solution (0.1 M phosphate buffer - $\mathrm{pH} 6.5,1.2 \mathrm{M}$ sorbitol). The ascus wall was digested by adding $1 \mu \mathrm{g} / \mu \mathrm{l}$ Zymolyase (Zymo Research) and an incubation at room temperature for 5 - $7 \mathrm{~min}$. The cells were washed once in $100 \mu \mathrm{l}$-solution and resuspended in $200 \mu \mathrm{l}$ solution. Of this cell suspension, $2.5-5 \mu \mathrm{l}$ were mixed with $100 \mu \mathrm{l}$ of water and pipetted on one third of a YPD plate. After drying, the tetrads were picked from the plate, using a tetrad microscope. After $2-3$ days of growth, the spores were restreaked on YPD plates and grown for another $2-3$ days to increase the cell material. Spores were kept in 96-well plates with $200 \mu \mathrm{l}$ of $50 \%$ glycerol per well. Cell material from all spores was stamped onto different YPD and selective plates to analyse the selection markers. The 96 well-plate was stored at $-80{ }^{\circ} \mathrm{C}$. All knockout strains contain a KanMX4 for selection. To select for this marker, $100 \mu \mathrm{l}$ of geneticin $(40 \mu \mathrm{g} / \mu \mathrm{l})$ was plated on a YPD plate.

For identification of the mating types, the spores were stamped on MATa and MATa reference strains (auxotrophic for valine and isoleucine). After one day of growth, the cells were transferred to selective B-plates (Sprague, 1991). 


\section{Colony PCR from yeast cells}

The knockouts in all used strains contain the same KanMX4 cassette. The combination of different knockouts was verified by colony PCR with primers that either amplified the wild typical gene or the reverse primer annealed in the KanMX4 gene and yielded an amplicon only in the knockout. In all cases, all four offspring strains from one tetrad were tested to verify the meiotic $2: 2$ segregation and specificity of the PCR.

Colony PCR samples were prepared by resuspending yeast cell material in PBS (137 mM $\mathrm{NaCl}, 2.7 \mathrm{mM} \mathrm{KCl}, 10 \mathrm{mM} \mathrm{KH}_{2} \mathrm{PO}_{4}, 2 \mathrm{mM} \mathrm{Na}_{2} \mathrm{HPO}_{4}$ ) and adding $3 \mu \mathrm{g} / \mu \mathrm{l}$ Zymolyase (Zymo Research). To completely digest the cell wall, the sample was incubated at $37^{\circ} \mathrm{C}$ for 1 hour, followed by $10 \mathrm{~min}$ at $95^{\circ} \mathrm{C}$. The cell debris was spun down by centrifugation at $16000 \mathrm{xg}$ for $1 \mathrm{~min}$. For one PCR (see 3.2.1), $1 \mu \mathrm{l}$ of the supernatant used as template DNA.

\subsubsection{Induction of galactose responsive promoters}

An inducible high transcription rate of reporter constructs was achieved with the GAL1 promotor. This promoter is induced by galactose and repressed by extracellular glucose (also in the presence of galactose). In raffinose or sucrose media, the GAL1 promotor is disinhibited, which leads to a constant transcription on a low level (Sellick et al., 2008).

For the galactose induction, the yeast cells were first grown in $5-20 \mathrm{ml}$ glucose containing medium for 1 day. The pre-culture was used to inoculate a culture with $2 \%$ raffinose or sucrose as its carbon sauce. The culture was grown for approx. $15 \mathrm{~h}$ until it reached an $\mathrm{OD}_{600}$ of $0.8-1.3$. The culture was diluted to $\mathrm{OD}_{600}: 0.8$ for a $2 \mathrm{~h}$ induction and $\mathrm{OD}_{600}: 0.5$ for a $4 \mathrm{~h}$ induction. The induction was started by adding 1/10 volume of $20 \%$ galactose. After 2 or $4 \mathrm{~h}$ of growth, the cells reached an $\mathrm{OD}_{600}$ of $1.0-1.3$ and were harvested.

In immunofluorescence experiments with expression of NMD reporters, the galactose induction was started at approx. $1.5 \times 10^{\wedge} 7 \mathrm{cells} / \mathrm{ml}$ and the cultures reached densities of $2-2.5 \times 10^{7}$ cells $/ \mathrm{ml}$ after $2 \mathrm{~h}$ of growth.

\subsection{Cell biology methods}

\subsubsection{Growth analysis of yeast strains}

Yeast cells from agar plates were resuspended in water and the density of the cell suspension was determined by cell counting (see 3.3.2). A dilution series of $10^{7}, 10^{6}, 10^{5}$, $10^{4}$ and $10^{3}$ cells $/ \mathrm{ml}$ was prepared for each strain. The dilution series was pipetted (in 10 $\mu$ l samples) in rows onto YPD plates. The cells were grown at $16^{\circ} \mathrm{C}, 25^{\circ} \mathrm{C}, 30^{\circ} \mathrm{C}$ or $37^{\circ} \mathrm{C}$ for $2-5$ days and images were taken by scanning. Data of growth analyses are not shown in the results section. However, the growth of crossed yeast strains and GFP fusion strains 
was tested to confirm that the strains have no growth defect (apart from a growth phenotype caused by $x r n 1 \Delta)$.

\subsubsection{Fluorescence microscopy}

For Hsp104-RFP microscopy, $10 \mathrm{ml}$ liquid cultures were grown to a cell density of $1-2$ $\mathrm{x} 10^{7} \mathrm{cells} / \mathrm{ml}$ and shifted to a temperature of $37^{\circ} \mathrm{C}$ in a water bath with light shaking for 1 h. For Gbp2-GFP and Hrb1-GFP microscopy with overexpression of an NMD reporter, 20 $\mathrm{ml}$ sucrose containing cultures were grown to $1-2 \times 10^{7} \mathrm{cells} / \mathrm{ml}$ and split in two. One half was induced with $2 \%$ galactose for $2 \mathrm{~h}$, while the other half was kept growing sucrose. In all fluorescence microscopy experiments the cells were fixated by adding $2.5 \%$ formaldehyde and harvested immediately. The cell pellet was washed once with $0.1 \mathrm{M}$ potassium-phosphate buffer - $\mathrm{pH} 6.5$ and once with $\mathrm{P}$-solution (0.1 M potassium phosphate buffer - pH 6.5, 1.2 M sorbitol) and resuspended in approx.10 $\mu \mathrm{l} \mathrm{P-solution} \mathrm{per} 10^{7}$ cells. To reduce bleaching of the fluorophores, the cells were kept in the dark as much as possible. Twelve-well microscope slides (5.2 mm wells; Thermo Scientific) were used for microscopy experiments. All washing and incubation steps were performed by pipetting 20 $\mu l$ of the respective solution or cell suspension onto one well and drawing it off with a vacuum pump. The microscope slides were prepared at room temperature. The wells were coated with a $0.3 \%$ poly-lysine solution. After $5 \mathrm{~min}$, the wells were washed once with water and air dried at $37^{\circ} \mathrm{C}$. The cell suspensions were incubated on the wells for $15 \mathrm{~min}$ and excess cell material was removed. To stain the nuclei, first, the cells were permeabilised by treatment with $0.5 \%$ Triton $\mathrm{X}-100$ (in $\mathrm{P}$-solution) for $1 \mathrm{~min}$, washed once with $\mathrm{P}$-solution and once with Aby wash 2 buffer $(0.1 \mathrm{M}$ Tris - $\mathrm{pH} 9.5,0.1 \mathrm{M} \mathrm{NaCl})$. The nuclei were stained with DAPI ( $1 \mu \mathrm{g} / \mathrm{ml}$ in Aby wash 2) for $5 \mathrm{~min}$, followed by three times washing with Aby wash 2 for $5 \mathrm{~min}$ each. The slides were air dried at $37^{\circ} \mathrm{C}$. Finally, all wells were covered in mounting medium (1 \% (w/v) n-propyl gallate, $40 \%$ (v/v) glycerol, $20 \%(\mathrm{v} / \mathrm{v})$ PBS: $137 \mathrm{mM}$ $\mathrm{NaCl}, 2.7 \mathrm{mM} \mathrm{KCl}, 10 \mathrm{mM} \mathrm{KH}_{2} \mathrm{PO}_{4}, 2 \mathrm{mM} \mathrm{Na}_{2} \mathrm{HPO}_{4}$ ), a cover slide was placed on top and light pressure was applied. The microscope slide was sealed with nail polish. The fluorescent signals were detected at a $63 x$ objective magnification and $1.6 x$ ocular magnification, using the following filter cubes (see Table 14). Recorded images were processed with ImageJ and Adobe Photoshop.

Table 14 - Filter cubes for fluorescence microscopy

\begin{tabular}{lllll} 
Fluorophore & Name & Excitation filter & Dichroic mirror & Emission filter \\
\hline GFP & L5 & BP480/40 & LP505 & BP527/30 \\
mRFP & TX2 & BP560/40 & LP595 & BP645/75 \\
DAPI & 405 & BP405/60 & LP455 & BP470/40 \\
DAPI & A4 & BP360/40 & LP400 & BP470/40
\end{tabular}




\subsection{Biochemical methods - protein analysis}

\subsubsection{Immunoprecipitation of GFP tagged proteins}

GFP fusion proteins were purified using GFP-Trap beads (Chromotek) or GFP-selector beads (Nanotag Biotechnologies), following the manufacturer's instructions with modifications. For one immunoprecipitation (IP) sample a $400 \mathrm{ml}$ culture was harvested. All preparation steps were performed in ice. The pellet was resuspended in the same volume $\left(500 \mu \mathrm{l}\right.$ ) of cooled PBSKMT buffer (PBS: $137 \mathrm{mM} \mathrm{NaCl}, 2.7 \mathrm{mM} \mathrm{KCl}, 10 \mathrm{mM} \mathrm{KH}_{2} \mathrm{PO}_{4}, 2 \mathrm{mM}$ $\mathrm{Na}_{2} \mathrm{HPO}_{4}$; with addition of $2.5 \mathrm{mM} \mathrm{MgCl}_{2}, 3 \mathrm{mM} \mathrm{KCl}$ and $0.5 \%$ Triton X-100) and protease inhibitor was immediately added $\left(5 \mu \mathrm{l}\right.$ per $100 \mu \mathrm{l}$ cell pellet; cOmplete ${ }^{\mathrm{TM}}$, EDTA-free Protease Inhibitor Cocktail, Roche). For cell lysis, 1 pellet-volume of glass beads (0.4 $0.6 \mathrm{~mm}$ ) was added and cells were lysed in a homogeniser at $4 \mathrm{~m} / \mathrm{s}$ for $30 \mathrm{~s}$ twice with $5 \mathrm{~min}$ on ice in between. Glass beads and cell debris were removed by centrifugation at $16000 \mathrm{x} \mathrm{g}$ for 5 min at $4{ }^{\circ} \mathrm{C}$ and the supernatant was transferred into a new tube. The supernatant was cleared further by centrifugation at $16000 \mathrm{x}$ for $10 \mathrm{~min}$ at $4^{\circ} \mathrm{C}$. Further, $10 \mu \mathrm{l}$ slurry of GFP Trap beads or GFP-selector beads were washed 3 times with $1 \mathrm{ml}$ PBSKMT buffer (after each washing step, the beads were centrifuged at $400 \mathrm{x} g$ for $1 \mathrm{~min}$ and the supernatant was removed). After centrifugation of the cell lysate, the supernatant was transferred into a new tube and a $20 \mu \mathrm{l}$ lysate sample was taken, mixed with $20 \mu \mathrm{l} 2 \mathrm{x}$ sample buffer (125 mM Tris - $\mathrm{pH}$ 6.8, 4 \% (w/v) SDS, $20 \%$ (v/v) glycerol, $0.05 \%$ (w/v) Bromophenol blue and $5 \%$ (v/v) 2-mercaptoethanol) and kept at $-20^{\circ} \mathrm{C}$ to be used as a lysate control for Western blot analysis. Approximately the rest of the cell lysate was added to the washed beads. In one experiment the same volume of lysate was used for IP for all samples. The lysate was incubated on the beads for $2 \mathrm{~h}$ on a rotator at $4{ }^{\circ} \mathrm{C}$. If indicated, $200 \mu \mathrm{g} / \mathrm{ml}$ RNase A were added after $1.5 \mathrm{~h}$ and RNA was digested for the remaining $30 \mathrm{~min}$. To wash the beads after the incubation, they were mixed with $1 \mathrm{ml}$ PBSKMT by inverting the tubes three times. Afterwards, the beads were centrifuged at $400 \mathrm{xg}$ for 1 min and the supernatant removed, leaving approx. $30 \mu \mathrm{l}$ of liquid in the tube. The beads were washed 5 to 7 times with PBSKMT. The number of washing steps was optimised for each IP individually. After the last washing step, the supernatant was removed as much as possible without losing beads, and for elution, $30 \mu \mathrm{l}$ sample buffer was added. The Eluate sample and the lysate sample was heated to $95^{\circ} \mathrm{C}$ and centrifuged briefly. The complete eluate and $20 \mu \mathrm{l}$ of the lysate sample were loaded on an SDS gel for Western blot analysis.

For formaldehyde crosslinking, $1 \%$ formaldehyde was added the yeast prior harvesting and the culture was incubated at $25^{\circ} \mathrm{C}$ for 10 min with shaking (protocol was adapted from Klockenbusch and Kast, 2010). The Formaldehyde was quenched by adding $0.5 \mathrm{M}$ glycine. 
The crosslinked immunoprecipitation was performed as described above. Before loading the samples on an SDS gel, they were heated to $95^{\circ} \mathrm{C}$ for 20 min for decrosslinking.

\subsubsection{SDS-PAGE}

Proteins were separated by size using standard, vertical, discontinuous SDS polyacrylamide gel electrophoresis (Garfin, 2009). All components of the separation gel (see Table 15, APS and TEMED were added last) were mixed and the gel was cast between two approx. $25 \times 20$ glass plates with approx. $2 \mathrm{~mm}$ thick spacers. The gel mix was covered with a layer of 2-propanol. After polymerisation, the 2-propanol was washed off with water and the water was removed. The components of the stacking gel were mixed (see Table 15, APS and TEMED were added last) and the stacking gel was cast on top of the separation gel. A comb was immediately inserted to form sample wells. After polymerisation of the stacking gel, the comb was removed and the gel placed into a gel-run chamber. The chamber contains two reservoirs with electrodes. The reservoirs are connected by the gel. Both reservoirs were filled with SDS running buffer (25 mM Tris Base, $0.1 \%$ (w/v) SDS, $190 \mathrm{mM}$ glycine). Remaining non-polymerised acrylamide was removed from the well using a syringe. The prepared samples, as well as a pre-stained protein marker (PageRuler Prestained Protein Ladder, Thermo Fisher Scientific or Cozy Prestained Protein Ladder, highQu), were pipetted into the wells. A power source was set to create a current of $14 \mathrm{~mA}$, with the negative electrode being on side of the loaded samples. The gel run was performed for approx. $15 \mathrm{~h}$.

Table 15 Composition of SDS polyacrylamide gels

\begin{tabular}{lll} 
Component & Stacking gel & Separation gel \\
\hline Bis-/Acrylamide mixture 37,5:1 & $5 \%(\mathrm{v} / \mathrm{v})$ & $10 \%(\mathrm{v} / \mathrm{v})$ \\
\hline Tris/HCl pH 8.8 & - & $0.375 \mathrm{mM}$ \\
Tris/HCl pH 6.8 & $125 \mathrm{mM}$ & - \\
\hline SDS & $0.1 \%(\mathrm{w} / \mathrm{v})$ & $0.1 \%(\mathrm{w} / \mathrm{v})$ \\
APS & $0.1 \%(\mathrm{w} / \mathrm{v})$ & $0.1 \%(\mathrm{w} / \mathrm{v})$ \\
\hline TEMED & $0.1 \%(\mathrm{v} / \mathrm{v})$ & $0.04 \%(\mathrm{v} / \mathrm{v})$
\end{tabular}

\subsubsection{Western blot analysis}

After SDS-PAGE, proteins were transferred to a nitrocellulose membrane $(9 \times 18 \mathrm{~cm})$ using a semi-dry blotting chamber (Alegria-Schaffer, 2014). The anode plate was covered with blotting buffer (25 mM Tris Base, $192 \mathrm{mM}$ glycine, $15 \%(\mathrm{v} / \mathrm{v})$ Methanol). The following components were shortly soaked in blotting buffer and stacked on the anode plate in this 
order: Whatman paper $(10 \times 20 \mathrm{~cm})$, membrane, SDS-Gel, Whatman paper. Air bubbles were removed, and additional buffer was poured on top until the stack was completely soaked in buffer. Finally, the cathode plate was placed on top and the proteins were transferred by applying $200 \mathrm{~mA}$ (approx. $1.2 \mathrm{~mA} / \mathrm{cm}^{2}$ ) for $1 \mathrm{~h} 45 \mathrm{~min}$.

After the transfer, the proteins were visualised by a short incubation in Ponceau $S$ solution $(0.2 \%(\mathrm{w} / \mathrm{v})$ Ponceau $S$ in $5 \%(\mathrm{v} / \mathrm{v})$ acetic acid), followed by rinsing with water. All following incubation or washing steps were performed on a shaker. The staining was completely removed by washing in TBS-T (50 mM Tris base, $150 \mathrm{mM} \mathrm{NaCL}, 0.1 \%$ (v/v) Tween 20). The membrane was blocked in $5 \%(\mathrm{w} / \mathrm{v})$ milk powder in TBS-T and incubated in the primary antibody solution for approx. $15 \mathrm{~h}$ at $4{ }^{\circ} \mathrm{C}$ (see Table 16, antibodies were diluted in TBS-T with $2 \%(\mathrm{w} / \mathrm{v})$ milk powder). The membrane was washed three times for 5 min with TBS-T and incubated in the secondary antibody solution (in TBS-T with $2 \%(\mathrm{w} / \mathrm{v})$ milk powder) for $2-4 \mathrm{~h}$. Again, the membrane was washed three times for 5 min with TBS-T. The membrane was rinsed several times with water and covered with ECL substrate solution (Amersham ECL, GE healthcare or WesternBright Quantum, Advansta). After $30 \mathrm{~s}$ of incubation, the signals were detected in a chemiluminescence imaging system. Quantification of Western blot signals were performed with Bio1d (Vilber Lourmat). Only signals that did not reach the detection limit in their intensity were quantified. The background signal on the membrane was subtracted.

Table 16 - Antibodies for Western blot analysis

\begin{tabular}{lll} 
Primary antibodies & Dilution & Source \\
\hline Mouse $\alpha$ GFP (monoclonal, GF28R) & $1 / 50000$ & Thermo Fischer Scientific \\
\hline Rabbit $\alpha$ GFP & $1 / 2000$ & Laboratory of Heike Krebber \\
Rabbit $\alpha$ Zwf1 & $1 / 50000$ & Courtesy of Roland Lill \\
\hline Mouse $\alpha$ Tdh1 (monoclonal, GA1R) & $1 / 50000$ & Thermo Fisher Scientific \\
\hline Rabbit $\alpha$ Hem15 & $1 / 5000$ & Courtesy of Roland Lill \\
\hline Rabbit $\alpha$ Gbp2 & $1 / 50000$ & Laboratory of Heike Krebber \\
Rabbit $\alpha$ Hrb1 & $1 / 20000$ & Laboratory of Heike Krebber \\
Mouse $\alpha$ c-MYC (monoclonal, 9E10) & $1 / 750$ & Santa Cruz Biotechnology \\
Mouse $\alpha$ HA (monoclonal, F-7) & $1 / 750$ & Santa Cruz Biotechnology \\
\hline Secondary antibodies & & \\
\hline Goat $\alpha$ Rabbit - HRP conjugated & Dilution & Source \\
\hline Goat $\alpha$ Mouse - HRP conjugated & $1 / 25000$ & Dianova \\
\hline
\end{tabular}

\subsubsection{Yeast cell lysis for Western blot analysis}

To analyse a whole cell lysate directly by Western blot analysis, the $\mathrm{OD}_{600}$ of a $50 \mathrm{ml}$ log phase yeast culture was measured and the cells harvested. The cells were lysed directly in 2x sample buffer (125 mM Tris - pH 6.8, 4 \% (w/v) SDS, $20 \%$ (v/v) glycerol, $0.05 \%$ (w/v) 
Bromophenol blue and $5 \%(\mathrm{v} / \mathrm{v}) 2$-mercaptoethanol; the volume was adjusted according to the measured $\left.\mathrm{OD}_{600}\right)$ with $200 \mu \mathrm{l}$ glass beads $(0.4-0.6 \mathrm{~mm})$ in a homogeniser at $4 \mathrm{~m} / \mathrm{s}$ for $30 \mathrm{~s}$ twice, with $5 \mathrm{~min}$ on ice in between. The samples were centrifuged at 16000x $\mathrm{g}$ for $1 \mathrm{~min}$ and the supernatant was transferred into a new tube. The samples were heated to 95 ${ }^{\circ} \mathrm{C}$ for $5 \mathrm{~min}$, centrifuged again for at $16000 \mathrm{xg} 1 \mathrm{~min}$ and $20 \mu \mathrm{l}$ were loaded on an SDS gel for Western blot analysis.

\subsection{Biochemical methods - RNA analysis}

\subsubsection{DEPC treatment of water}

RNases in water were inactivated by addition of $0.1 \%(\mathrm{v} / \mathrm{v})$ diethyl pyrocarbonate (DEPC) and incubation at room temperature for approx. $15 \mathrm{~h}$ with stirring. Remaining DEPC was inactivated by autoclaving $\left(121^{\circ} \mathrm{C}, 20 \mathrm{~min}\right)$.

\subsubsection{RNA Co-Immunoprecipitation with GFP-tagged proteins}

\section{Co-Immunoprecipitation}

To analyse the association of RNAs with a GFP-tagged protein, a regular Immunoprecipitation (IP, compare 3.5.1) was performed, followed by RNA isolation. For one RNA co-Immunoprecipitation (RIP) sample $400 \mathrm{ml}$ yeast culture was harvested after $2 \mathrm{~h}$ galactose induction (see 3.3.5). Protein-RNA complexes were crosslinked by UV irradiation (described in Sei and Conrad, 2014). The cell pellet was resuspended in $50 \mathrm{ml}$ of water and transferred into a $15 \mathrm{~cm}$ petri dish. The petri dish was placed on a cooled metal block in a UV chamber. The cells were exposed two times for $3.5 \mathrm{~min}\left(0.6 \mathrm{~J} / \mathrm{cm}^{2}\right)$ to $254 \mathrm{nM}$ UV light, with light shaking in between for a better heat distribution and crosslink efficiency. The cell pellet was lysed in 2x pellet-volume of RIP-buffer (150 mM NaCl, $2 \mathrm{mM} \mathrm{MgCl}_{2}, 0.2 \mathrm{mM}$ PMSF, $0.5 \mathrm{mM}$ DTT, $0.2 \%$ (v/v) Triton X-100, $25 \mathrm{mM}$ Tris/HCl - $\mathrm{pH}$ 7.5), protease inhibitor $\left(5 \mu \mathrm{l}\right.$ per $100 \mu \mathrm{l}$ cell pellet; cOmplete ${ }^{\mathrm{TM}}$, EDTA-free Protease Inhibitor Cocktail, Roche), RNase inhibitor $(0.6 \mu \mathrm{l} / 500 \mu \mathrm{l}$ pellet-volume RiboLock, Thermo Scientific) and $1 \mathrm{x}$ pelletvolume of glass beads $(0.4-0.6 \mathrm{~mm})$. The lysis was performed twice at $4 \mathrm{~m} / \mathrm{s}$ for $30 \mathrm{~s}$ in a homogeniser, with $5 \mathrm{~min}$ on ice between lysis steps. Glass beads and cell debris were removed by centrifugation at $16000 \mathrm{xg}$ for $1 \mathrm{~min}$ at $4{ }^{\circ} \mathrm{C}$ and the supernatant was transferred into a new tube. The supernatant was cleared further by centrifugation at $16000 \mathrm{x} \mathrm{g}$ for 10 min at $4{ }^{\circ} \mathrm{C}$. Further, $10 \mu \mathrm{l}$ slurry of GFP-Selector beads (Nanotag Biotechnologies) were washed 3 times with RIP buffer. The beads were washed by adding $1 \mathrm{ml}$ buffer, inverting the tube three times, 1 min centrifugation at $400 \mathrm{xg}$, and removal of the supernatant. After centrifugation of the cell lysate, the supernatant was transferred into a new tube and a $20 \mu \mathrm{l}$ lysate sample was taken, mixed with $20 \mu \mathrm{l} 2 \mathrm{x}$ sample buffer (125 mM Tris - $\mathrm{pH}$ 6.8, 4 \% 
(w/v) SDS, $20 \%(\mathrm{v} / \mathrm{v})$ glycerol, $0.05 \%(\mathrm{w} / \mathrm{v})$ Bromophenol blue and $5 \%(\mathrm{v} / \mathrm{v}) 2$ mercaptoethanol) and kept at $-20^{\circ} \mathrm{C}$ to be used as a lysate control for Western blot analysis. Further, $100 \mu \mathrm{l}$ of lysate were transferred into a new tube as a whole-cell lysate sample for RNA purification (lysate sample). Approx. the rest of the cell lysate was added to the washed beads (RIP sample), equal volumes were loaded for all samples. DNasel was added to the lysate sample (14 Kunitz units to $100 \mu \mathrm{l}$ sample) and to the RIP sample (6.5 Kunitz units per $100 \mu \mathrm{l})$. The lysate and RIP samples were incubated for $2 \mathrm{~h}$ on a rotator at $4{ }^{\circ} \mathrm{C}$. Afterwards, the lysate samples were kept on ice, the RIP samples were washed 5 times in RIP-buffer and washed two more times in Proteinase $\mathrm{K}$ buffer $(50 \mathrm{mM} \mathrm{NaCl}, 0.5 \mathrm{mM}$ DTT, $0.2 \%$ Triton $\mathrm{X}-100,50 \mathrm{mM}$ Tris/ $\mathrm{HCl} \mathrm{pH} 7.5$ in DEPC treated water). The beads were washed by adding $1 \mathrm{ml}$ buffer, inverting the tube three times, and $1 \mathrm{~min}$ centrifugation at $400 x \mathrm{~g}$. The supernatant was removed, leaving approx. $30 \mu \mathrm{l}$. In the last washing step, the beads were mixed with $1 \mathrm{ml}$ buffer, and $200 \mu \mathrm{l}$ of the bead suspension were transferred into a new tube prior to centrifugation to be used for Western blot analysis. After the last centrifugation step, the supernatant of the Western blot sample was removed as much as possible without loss of beads and $30 \mu \mathrm{l} 2 \mathrm{x}$ sample buffer were added. The sample was kept at $-20{ }^{\circ} \mathrm{C}$ until it was used for Western blot analysis (see 3.5.2; 3.5.3). For the RIP sample, approx. $50 \mu$ l supernatant was left on the beads, 3 Kunitz units of DNasel and 0.5 $\mu \mathrm{l}$ RNase inhibitor (RiboLock, Thermo Scientific) were added. The RIP and Lysate samples were incubated at $25{ }^{\circ} \mathrm{C}$ on a rotator for further DNase digestion. Afterwards, $0.5 \%$ SDS and $5 \mathrm{mM}$ EDTA was added to Lysate and RIP samples. Proteins were digested by addition of $80 \mu \mathrm{g}$ (Lysate samples) or $40 \mu \mathrm{g}$ (RIP samples) Proteinase $\mathrm{K}$ and 90 min incubation at $55^{\circ} \mathrm{C}$ with shaking.

The eIF4E RIPs were washed more stringently to remove RNA that is not crosslinked. The samples were first washed with three times with RIP-buffer that had $0.5 \%$ Triton X-100 (instead of $0.2 \%$ ), three times with RIP-buffer, to which $1 \mathrm{M} \mathrm{NaCl}$ was added, and three times with Proteinase $\mathrm{K}$ buffer.

\section{RNA isolation}

RNA from lysate and eluate samples was purified using TRlzol reagent (Thermo Fisher Scientific), following the manufacturer's instructions (with modifications). For this, $1 \mathrm{ml}$ TRIzol was added to the sample and the sample was incubated at $65^{\circ} \mathrm{C}$ for 10 min with shaking, and $200 \mu \mathrm{l}$ Chloroform were added. The samples were mixed thoroughly and centrifuged at $16000 \mathrm{xg}$ for $15 \mathrm{~min}$. The aqueous phase was transferred into a new tube and mixed with the same volume 2-propanol. Next, $20 \mu \mathrm{g}$ glycogen or $15 \mu \mathrm{g}$ GlycoBlue (Thermo Fisher Scientific) (for lysate and eluate samples, respectively) were added, and the sample was mixed and incubated for approx. $15 \mathrm{~h}$ at $-20{ }^{\circ} \mathrm{C}$. Precipitated RNA was 
pelleted by 30 min centrifugation at $16000 \mathrm{xg}$ and $4{ }^{\circ} \mathrm{C}$. The supernatant was removed. For complete removal of the supernatant, the sample was centrifuged briefly again and remaining supernatant was removed. The pellet was washed with $75 \%$ ethanol (pre-cooled to $-20^{\circ} \mathrm{C}$ ). The centrifugation and washing procedure was repeated once. After a final centrifugation, the ethanol was removed completely, and the pellet was dried for $5 \mathrm{~min}$ at $65^{\circ} \mathrm{C}$. The pellet was resolved in $20 \mu \mathrm{LEPC}$ treated water at $65^{\circ} \mathrm{C}$ for $10 \mathrm{~min}$ with shaking. Lysate samples were further digested with DNase for $30 \mathrm{~min}$, using the Turbo DNAfree kit, following the manufacturer's instructions. RNA concentrations were measured using a NanoDrop spectrophotometer (see 3.2.6).

\subsubsection{RNA isolation from whole-cell lysates}

RNA from whole cell lysates (except lysate samples in RNA co-Immunoprecipitation experiments) was purified with the silica membrane based NucleoSpin RNA kit (MachereyNagel), following the manufacturer's instructions, with the following modifications: the cells were lysed twice at $4 \mathrm{~m} / \mathrm{s}$ for $30 \mathrm{~s}$ in a homogeniser, with $5 \mathrm{~min}$ on ice between lysis steps; the DNase digest was extended from $15 \mathrm{~min}$ to $30 \mathrm{~min}$ for better DNA removal.

\subsection{4. cDNA synthesis from RNA}

To synthesise cDNA from an RNA sample, 100 ng eluted RNA from RNA CoImmunoprecipitation or $1 \mu \mathrm{g}$ RNA from whole-cell lysates was reverse transcribed. Either the Maxima First Strand cDNA Synthesis Kit (Thermo Fisher Scientific) was used with Oligo $(\mathrm{dT})_{18}$ primers, following the manufacturer's instructions (RNA and primers were not preincubated at $65{ }^{\circ} \mathrm{C}$ and reverse transcription was performed at $50{ }^{\circ} \mathrm{C}$ ), or the FastGene Scriptase II Kit (NIPPON Genetics) was used, following the manufacturer's instructions, with random hexamer primers and a reverse transcription temperature of $42{ }^{\circ} \mathrm{C}$.

After reverse transcription, the cDNA was diluted 1:5-1:500 with DEPC treated water. The dilution was optimised target specifically to be suitable for qPCR analysis. For each cDNA sample, a -RT control was prepared in the same way, without addition of the reverse transcriptase.

\subsubsection{Quantitative Polymerase Chain Reaction (qPCR)}

All qPCR samples, except the -RT controls, were prepared at least in triplicates. One qPCR was performed in $10 \mu$ l containing $5 \mu$ l qPCR master mix (qPCRBIO SyGreen Mix Lo-ROX, Nippon Genetics; the protocol is based on the provided instructions), $80-240 \mathrm{nM}$ forward and reverse primers, and $2 \mu \mathrm{l}$ cDNA. A two-step protocol was used with min $95^{\circ} \mathrm{C}$ initial 
denaturation and $40-45$ cycles with $30 \mathrm{~s}$ at $60^{\circ} \mathrm{C}$ and $10 \mathrm{~s}$ at $95^{\circ} \mathrm{C}$. SYBR Green fluorescence was measured at the end of each $60{ }^{\circ} \mathrm{C}$ step. Primer concentrations were optimised for each target to reach amplification efficiencies of $0.8-1$. Primer pairs were designed with Primer-BLAST (NCBI).

\subsection{Statistical analysis}

All experiments were performed in at least three independent biological replicates. All error bars show the standard deviation of biological replicates. To determine the statistical significance, $p$-values were calculated by unpaired, two tailed, homo- or heteroscedastic Student's t-test. The $p$-values are indicated by * $(p<0.05),{ }^{* *}(p<0.01)$ and ${ }^{* * *}(p<0.001)$. In bar diagrams, in which the values were normalised, asterisks above the bars indicate a statistically significant difference to 1 (100\%). Additionally, significant differences between two normalised values are indicated with horizontal brackets. In the quantification of observed phenotypes in fluorescence microscopy experiments, asterisks above bars indicate a statistically significant difference to the wild type sample. Significant differences between other samples are indicated with horizontal brackets. 


\subsection{Cloned plasmids}

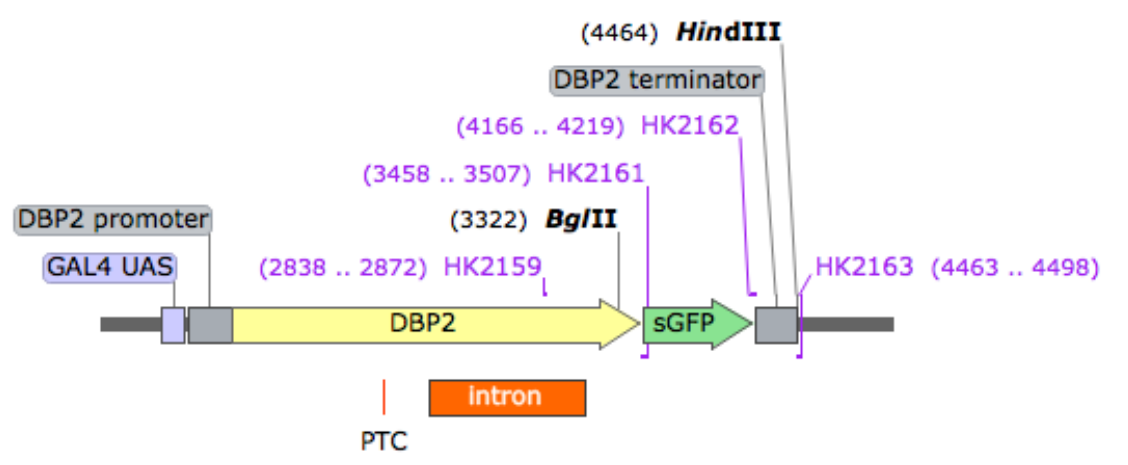

pHK1551 - GAL4-UASDBP2 ${ }^{\text {PTC }}$-GFP: Part of the DBP2PTC ORF was amplified by PCR from pHK1312 with the primer pair HK2159/HK2161 and the terminator with the primer pair HK2162/HK2163. The GFP sequence was removed from pHK1333 by cleavage with Xhol and BamHI. All three fragments were inserted by Gibson Assembly into pHK1312, which was cleaved by Bglll and BamHI. Note: the Xhol and BamHI site were destroyed in the Gibson Assembly.

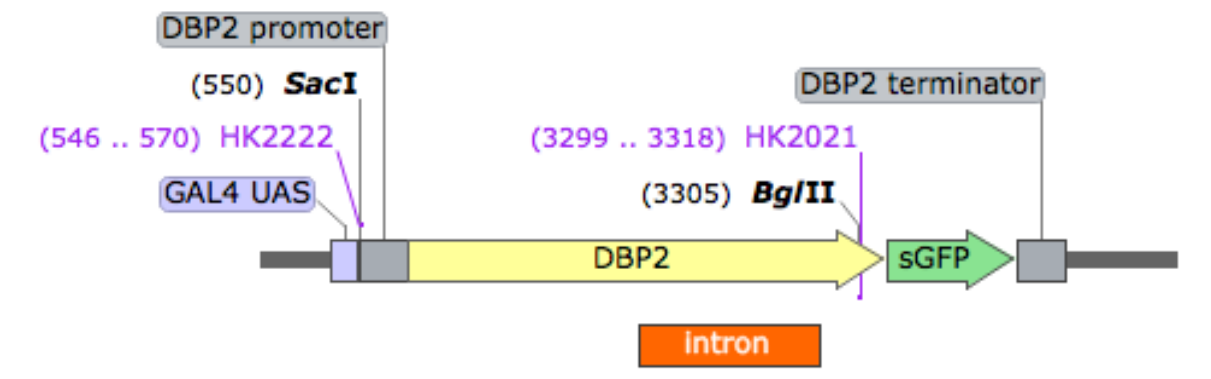

pHK1570 - GAL4-UASDBP2-GFP: The DBP2 promotor and wild typical ORF were amplified by PCR from yeast genomic DNA with the primer pair HK2222/HK2021, and ligated downstream of the GAL4-UAS of pHK1551, which was cleaved by Sacl and Bglll. 


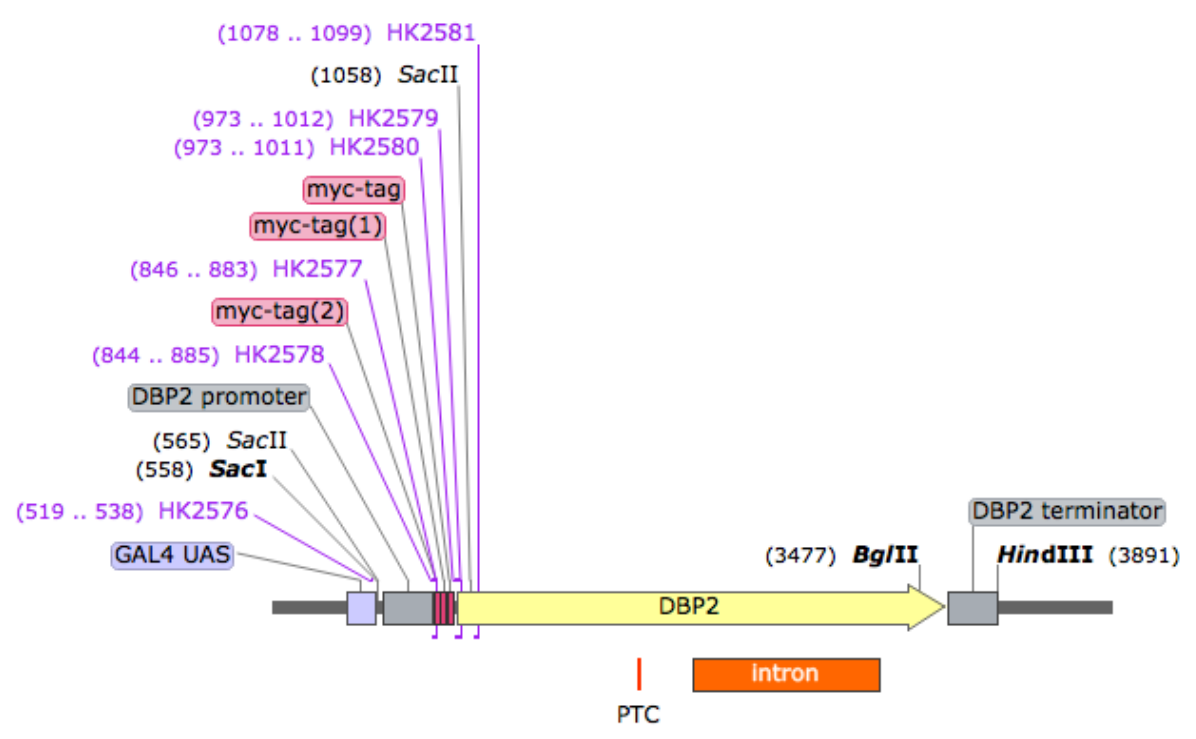

PHK1577 - GAL4-UASMYC-DBP2 ${ }^{\text {PTC: }}$ The DBP2 promoter were amplified by PCR from pHK1551 with the primer pair HK2576/HK2577. The MYC sequence was amplified from pHK1571 with the primer pair HK2578/HK2579. The DBP2PTC ORF was amplified from pHK1551 with the primer pair HK2580/HK2581. The inserts were assembled by Gibson Assembly with pHK1551, which was cleaved by Sacl and Sacll. The GFP tag from pHK1551 was removed by cleavage with BgIII and HindIII and ligating the plasmid with a BgIII-HindIII fragment from pHK1312.

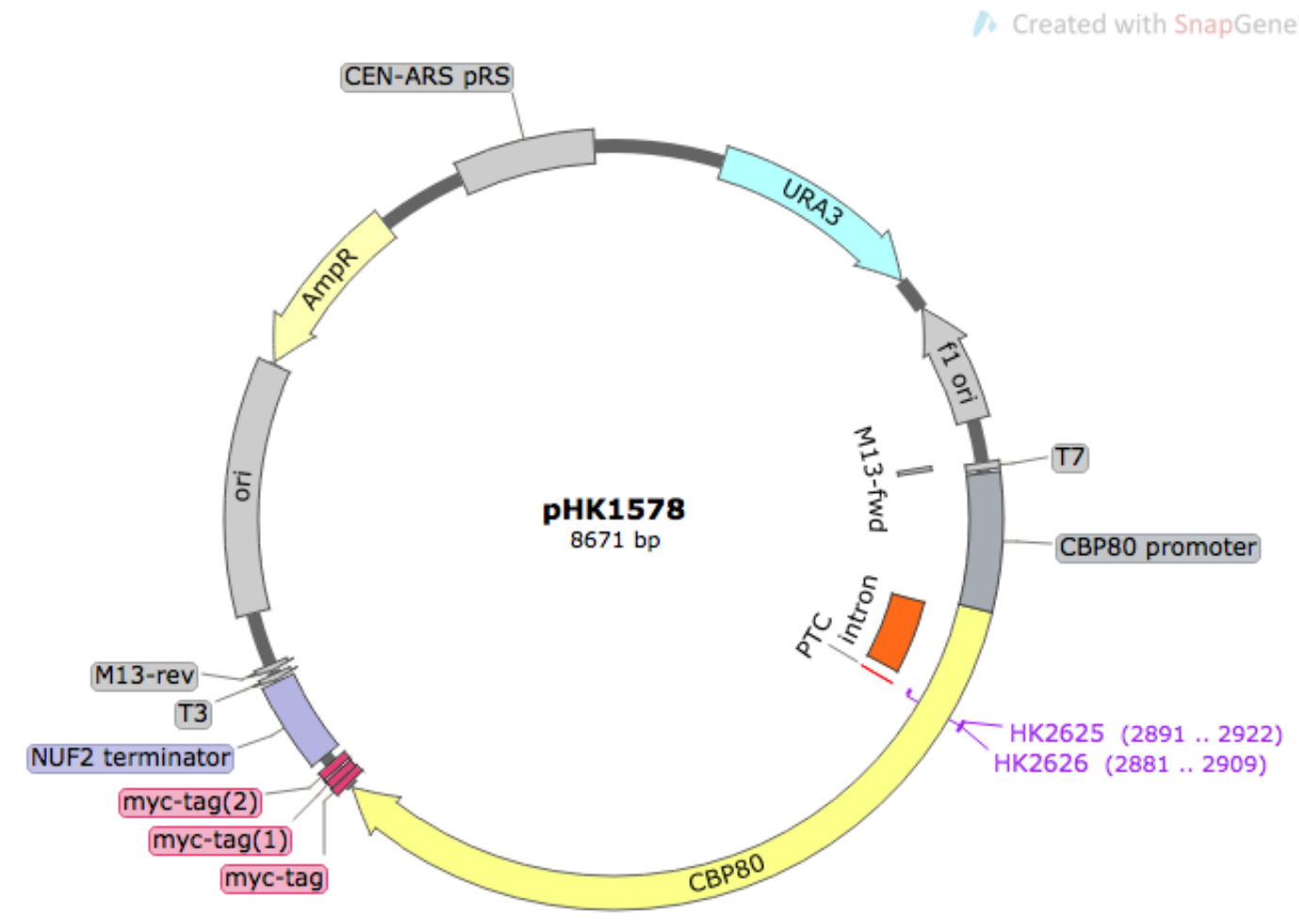

pHK1578 - CBP80 ${ }^{\text {PTC }}$-MYC: The premature stop codon was introduced into the CBP80 ORF by site directed mutagenesis, amplifying the CBP80-MYC plasmid pHK1574 with the mutagenesis primers HK2625/HK2626. 


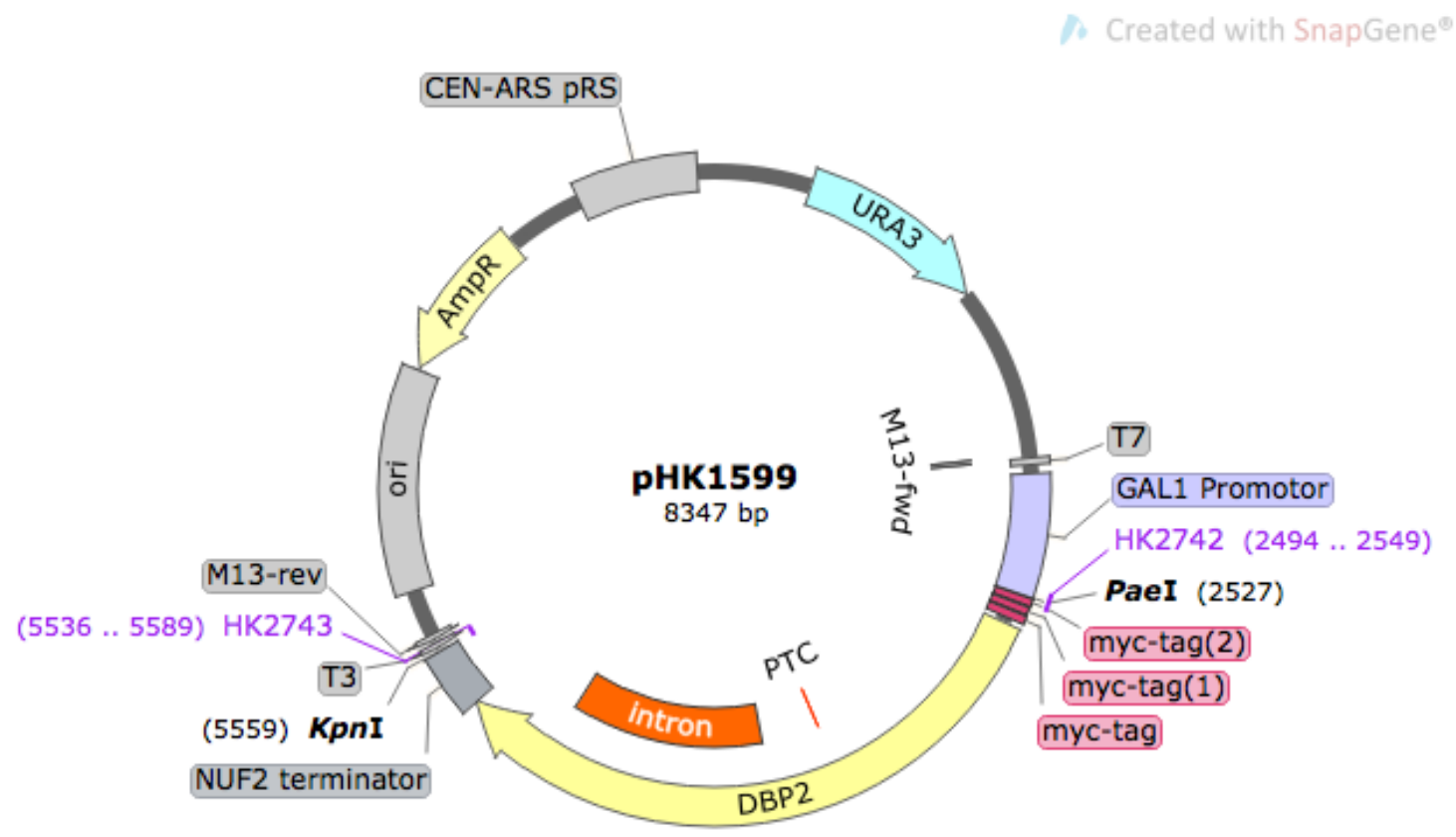

pHK1599 - GAL1 MYC-DBP2 ${ }^{\text {PTC: }}$ The MYC-DBP2PTC ORF, together with the NUF2 terminator sequence, were amplified by PCR with the primer pair HK2742/HK2743. The fragment was inserted by Gibson Assembly into pHK385, which was cleaved by EcoRI and Kpnl. Note: the EcoRI site was altered into a Pael site.

\section{Created with SnapGene ${ }^{\bullet}$}

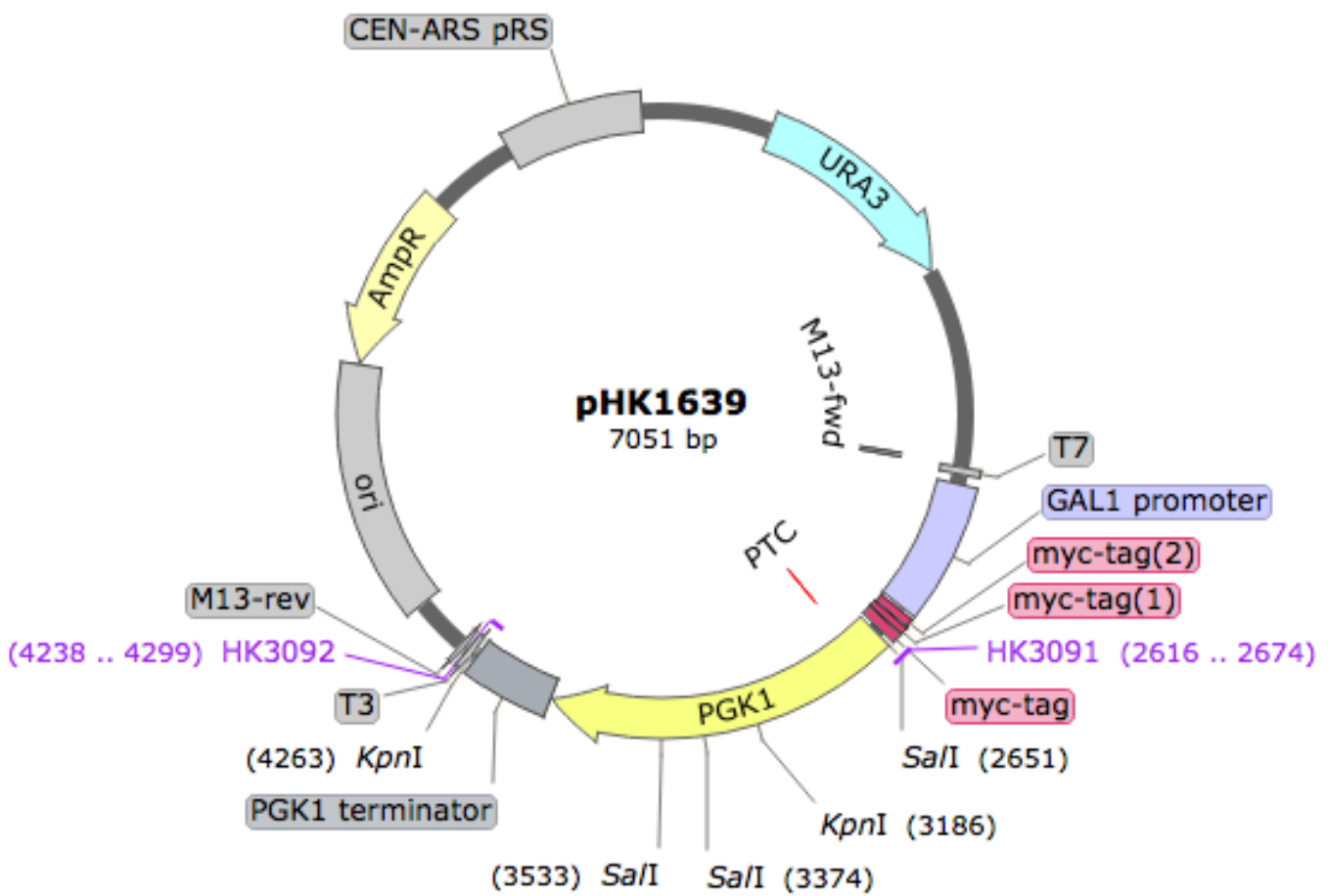

pHK1639 - GAL1MYC-PGK1 ${ }^{\text {PTC: }}$ : The PGK1 ${ }^{\text {PTC }}$ ORF and terminator sequence was amplified by PCR from pHK657 with the primer pair HK3091/HK3092, and inserted by Gibson Assembly into pHK1600, which was cleaved with Sacl and Kpnl. 


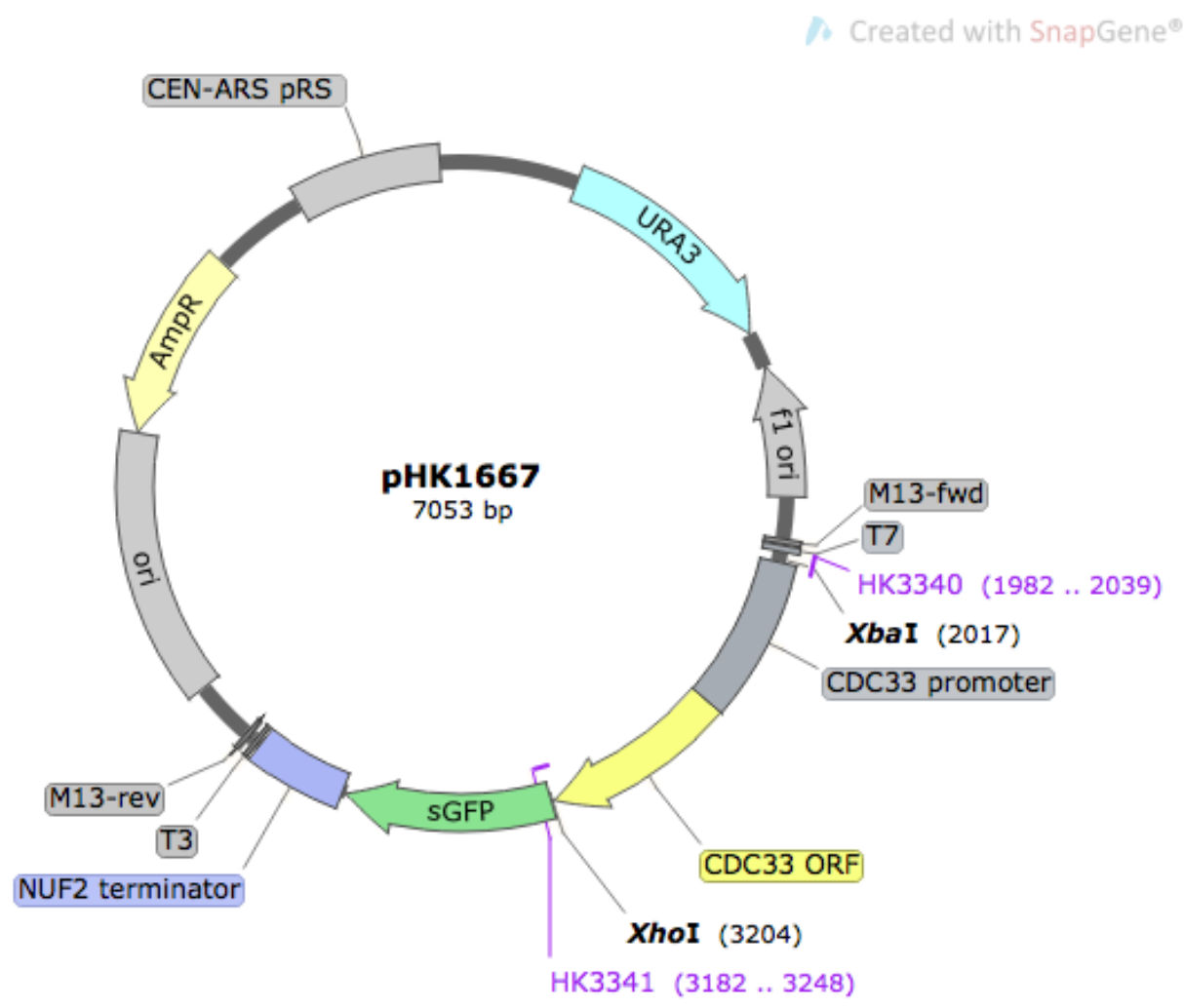

pHK1667 - CDC33-GFP: The CDC33 promoter and ORF sequence was amplified by PCR from yeast genomic DNA with the primer pair HK3340/HK3341, and inserted by Gibson Assembly into pHK12, with was cleaved with Xhol and Xbal.

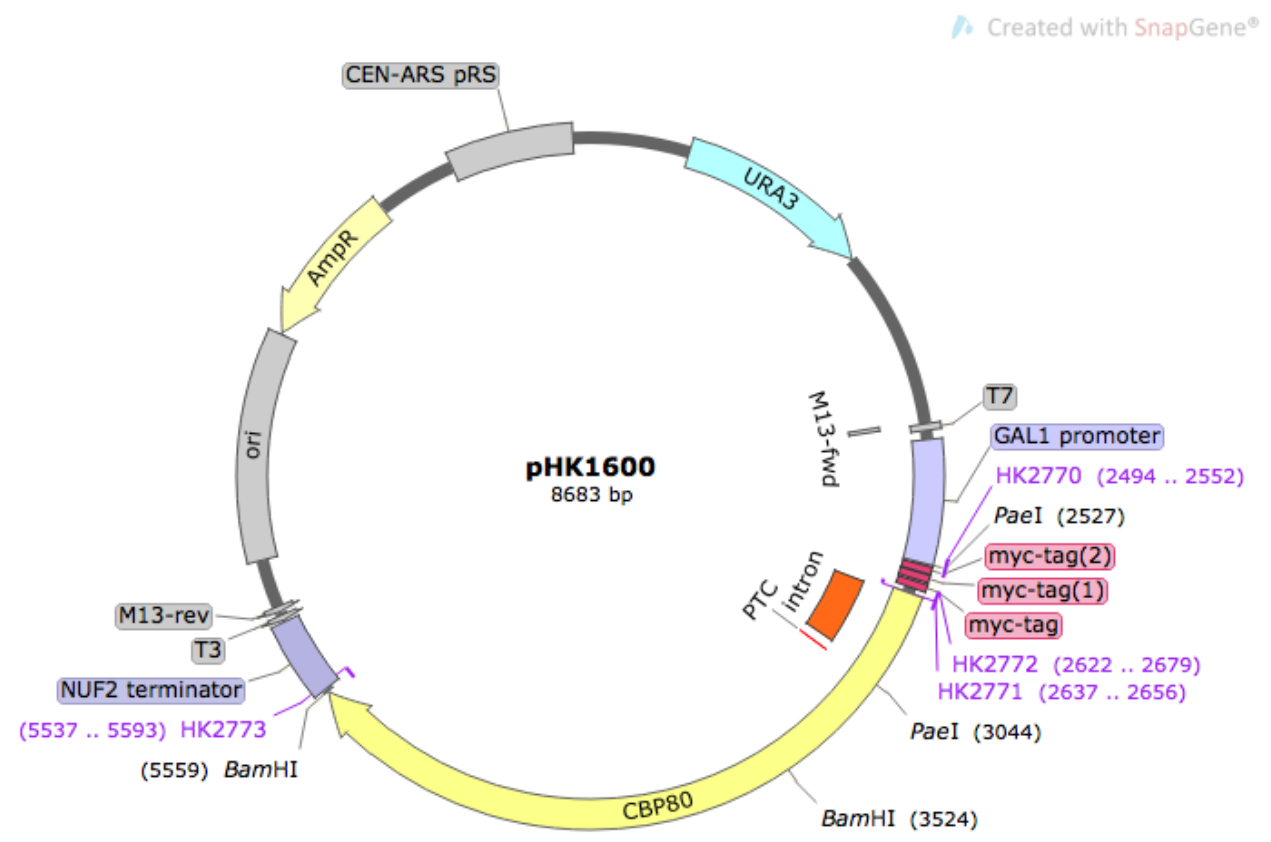

PHK1600 - GAL1MYC-CBP80 ${ }^{\text {PTC: }}$ : The MYC sequence was amplified by PCR from pHK1578 with the primer pair HK2770/HK2771. The CBP80 PTC ORF was amplified with the primer pair HK2772/HK2773. Both fragments were inserted by Gibson Assembly, into the plasmid pHK385, from which the original ORF was removed by cleavage with EcoRI and BamHI. Note: the EcoRl site was altered to a Pael site. 


\section{Results}

\subsection{Gbp2 and Hrb1 are novel factors in cytoplasmic quality control}

We knew that Gbp2 and Hrb1 are part of translated mRNPs in the cytoplasm and they are quality control factors in the nucleus. Here we addressed the question whether they are also cytoplasmic quality control factors during translation.

It was recently published that mutants of cytoplasmic quality control factors lead to protein aggregation. Hsp104-RFP forms visible foci in these mutants (Jamar et al., 2018). To test whether Gbp2 and Hrb1 might continue their quality control function in the cytoplasm, we

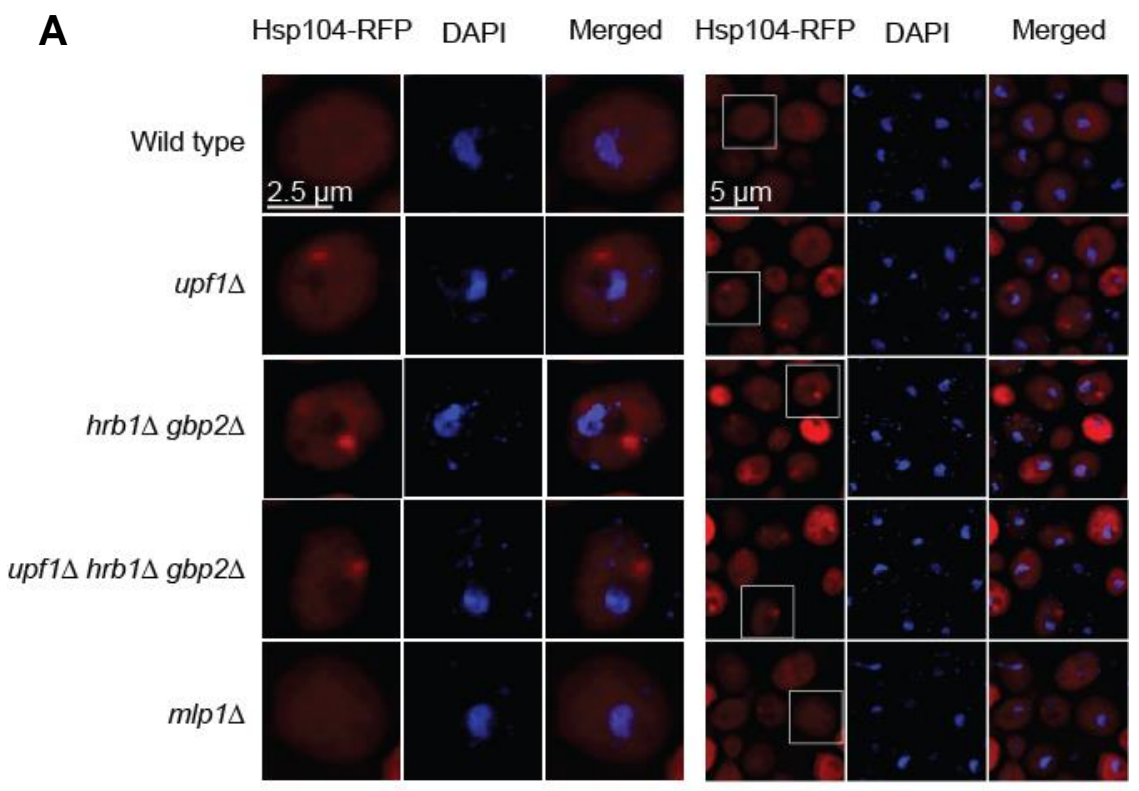

B

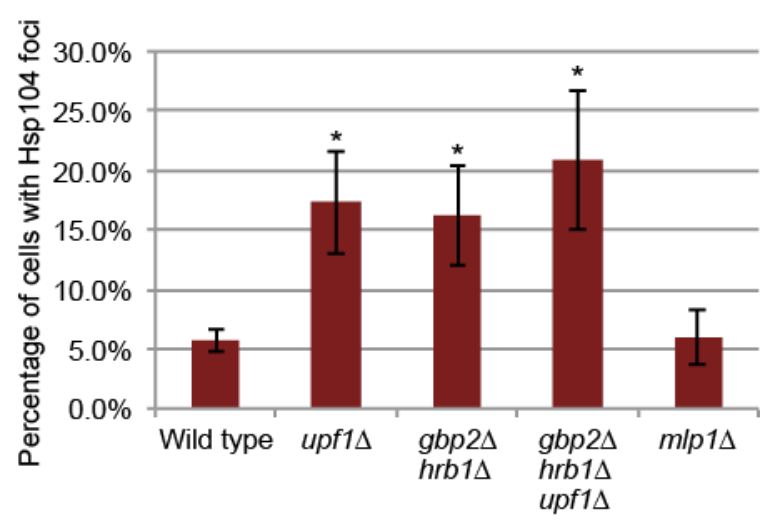

Figure 7: Hsp104-RFP aggregates in mutants of cytoplasmic quality control.

A Single cell and overview from fluorescence microscopy of indicated strains expressing HSP104-RFP. B Quantification of cells showing Hsp104-RFP foci in (A). For each strain, at least 1000 cells were analysed. Error bars represent the standard deviation between experiments with approx. 300 analysed cells per experiment per strain. $n=3$ (for wild type and $u p f 1 \Delta \mathrm{n}=6$ ) 
analysed the Hsp104-RFP localisation in gbp2 $\Delta$ hrb1 $\Delta$ cells. Interestingly, $16 \%$ of the cells showed Hsp104-RFP foci, which was comparable to a upf1 $\Delta$ strain( 17\%, see Figure 7). In contrast, only $5 \%$ of wild type cells showed Hsp104-RFP foci. To test whether an

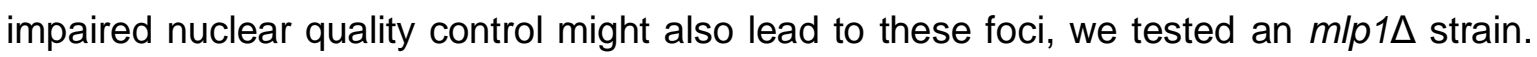
Mlp1 of the nuclear basket is very likely exclusively nuclear, and thus, only affects nuclear events directly. Similar to $g b p 2 \Delta h r b 1 \Delta$, mlp1 $1 \Delta$ cells show a leakage of unspliced pre-

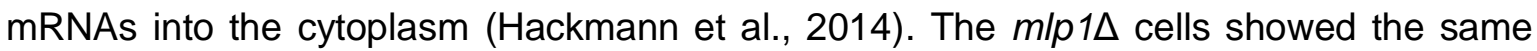
phenotype as wild type cells, suggesting that mutants of nuclear quality control do not cause protein aggregation. This result is a first indication that Gbp2 and Hrb1 are indeed involved in cytoplasmic quality control. Further, the combination of gbp2 $h r b 1 \Delta$ upf1 $\Delta$ showed, on average, only a mildly stronger phenotype. This suggests that Gbp2 and Hrb1 may, at least partially, function in the same pathway as Upf 1.

\subsection{Upf1 mediated degradation of NMD targets is defective in gbp2 $\Delta$ hrb1 $\Delta$ cells}

Once we had the first indication that Gbp2 and Hrb1 are part of the cytoplasmic quality control and potentially cooperating with Upf1, we tested if Gbp2 and Hrb1 are indeed relevant for NMD. For this, we used PTC (Premature termination codon) containing reporter constructs. The most widely used NMD reporter is based on the PGK1 gene. Previous analysis showed, however, no influence of Gbp2 and Hrb1 on the mRNA level of this reporter (unpublished data, laboratory of Heike Krebber). It was found out later that Gbp2 and Hrb1 have a nuclear quality control function on spliced mRNAs. As they also have a preferential binding to RNAs from intron containing genes, they might generally rather be relevant for this subgroup of transcripts. Also, as mis- or unspliced transcripts are thought to be a major source of NMD targets, it is especially interesting to investigate the role of Gbp2 and Hrb1 in NMD for spliced transcripts. To test if Gbp2 and Hrb1 might be required for NMD on such transcripts, we constructed two reporters based on the intron containing genes $C B P 80$ and $D B P 2$ (see Figure $8 \mathrm{~A}$ and Figure $9 \mathrm{~A}$ ). CBP80 is a typical spliced mRNA in yeast, as the intron is very close to the start codon. The PTC is shortly downstream of the splice site. Naturally occurring mis- or unspliced transcripts that leak into the cytoplasm, would typically have a PTC close to the start codon (see Saccharomyces Genome Database - Christie et al., 2004). The DBP2 reporter reflects the condition, which is described for the Exon Junction Complex in higher eukaryotes (Brogna and Wen, 2009). The PTC is upstream of the exon junction. As it is known that Gbp2 and Hrb1 have a nuclear quality control function on spliced mRNAs, thus, it is necessary to distinguish between nuclear and cytoplasmic effects. For this, we used control reporters without a PTC. As the 
PTC is frame dependent, it can only be recognised during translation. Hence, any effect by addition of a PTC must be co-translational. As expected, for both reporters we could see that a PTC reduces the RNA levels of the reporter constructs (to $~ 45 \%$ for CBP80 and $65 \%$ for DBP2 see Figure $8 \mathrm{~B}$ and Figure $9 \mathrm{~B}$ ).

Interestingly, we could see that this effect is considerably reduced in gbp2 $\Delta$ hrb1 $\Delta$ cells. The CBP80 mRNA level was not influenced by a PTC in ufp1 $1 \Delta$ cells and the additional loss of Gbp2 and Hrb1 show no further effect, indicating that Gbp2 and/or Hrb1 are involved in the Upf1 mediated and PTC induced destabilisation of NMD targets. Surprisingly, the DBP2 mRNA showed elevated RNA levels by addition of a PTC in the mutant strains. However, when we compare how much the PTC containing NMD reporters are influenced by the absence or presence of Upf1, both reporters show that the Upf1 mediated destabilisation is reduced approx. by half without Gbp2 and Hrb1. In the presence of Gbp2 and Hrb1, functional Upf1 reduces the levels of both reporters to $40-50 \%$, but only to $78 \%$ (on average) if they are knocked out (see Figure 10). The results suggest that Gbp2 and/or Hrb1 are required for the effective degradation of spliced NMD targets caused by Upf1.
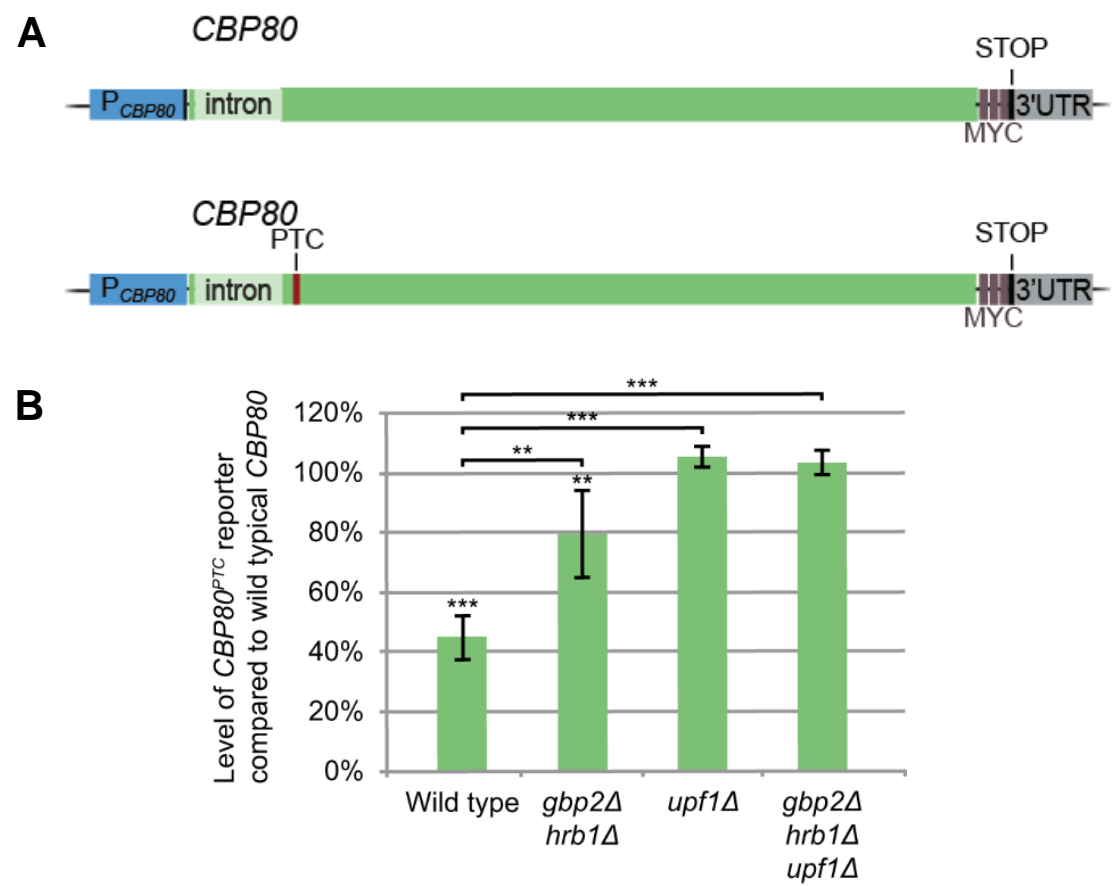

Figure 8: Gbp2 and Hrb1 are required for PTC induced destabilisation of CBP80 mRNA.

A Sketch of CBP80 reporter constructs with and without a premature termination codon (PTC). B RNA levels of CBP80 ${ }^{\text {PTC }}$ in percent of wild typical CBP80. RNA was isolated from indicated strains containing the control (wild typical) or NMD reporter. RNA levels of reporter constructs were quantified by $\mathrm{qPCR}$. The MYC sequence was used to discriminate between reporters and endogenous CBP 80 mRNA. In each experiment the NMD reporter sample was normalised to the control reporter sample of the same mutant. ( $n=4$; for gbp2 $n=7$ ) 


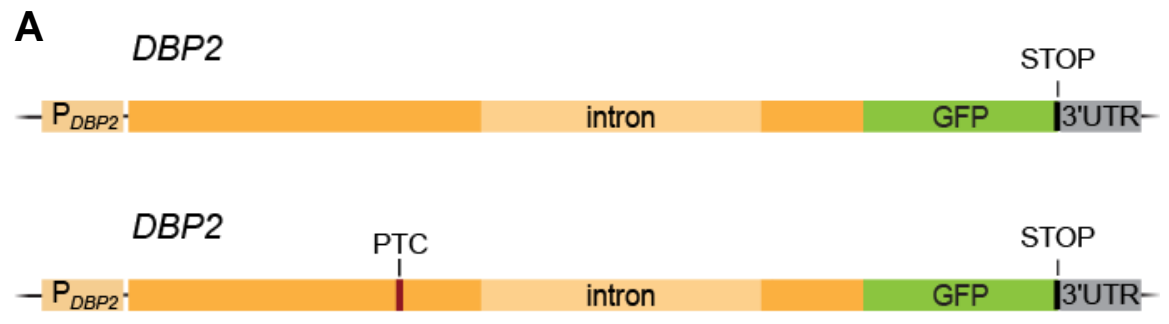

B

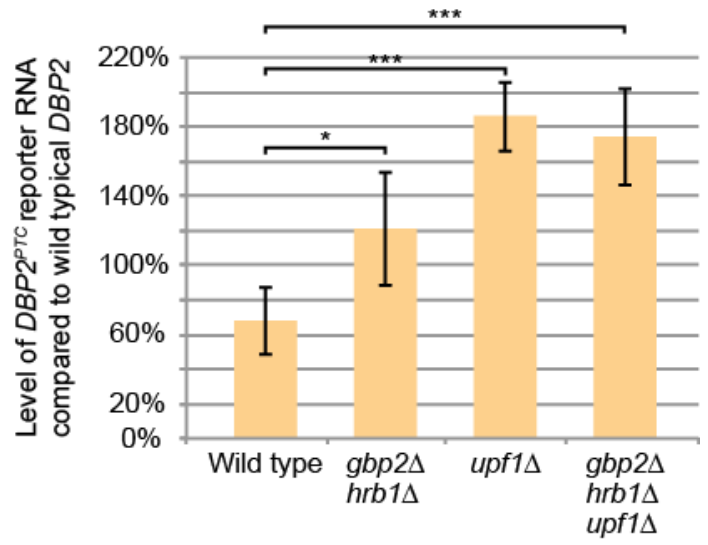

Figure 9: Gbp2 and Hrb1 are required for PTC induced destabilisation of DBP2 mRNA.

A Sketch of $D B P 2$ reporter constructs with and without a premature termination codon (PTC). B RNA levels of DBP2 ${ }^{P T C}$ in percent of wild typical DBP2. RNA was isolated from indicated strains containing the control (wild typical) or NMD reporter. RNA levels of reporter constructs were quantified by qPCR. The GFP sequence was used to discriminate between reporters and endogenous $D B P 2$ mRNA. In each experiment the NMD reporter sample was normalised to the control reporter sample of the same mutant. $(n=4)$

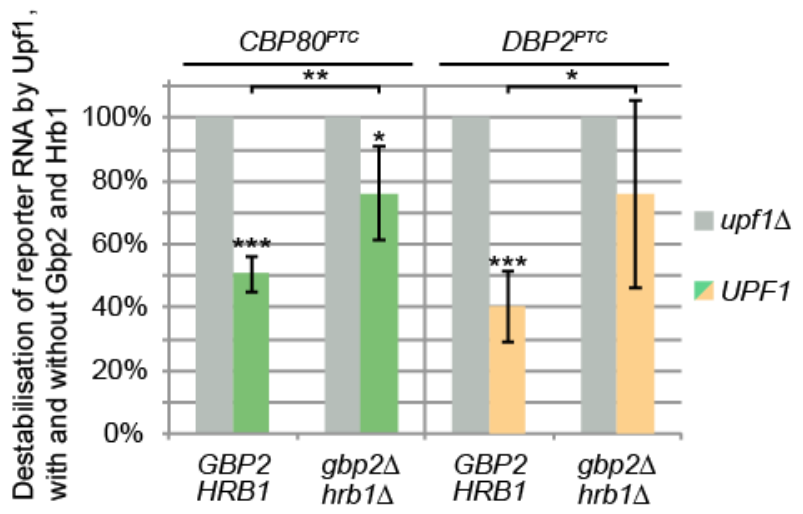

Figure 10: Gbp2 and Hrb1 are involved in the Upf1 mediated destabilisation of the NMD reporters.

NMD reporter levels from experiments in Figure 8 and Figure 9. In each experiment the NMD reporter level of wild type cells was normalised to the upf1 $\Delta$ level and the gbp2 $\Delta$ hrb1 $\Delta$ level was normalised to the upf1 $g b p 2 \Delta$ hrb1 1 level. $\left(C B P 80^{P T C}: \mathrm{n}=3, D B P 2^{P T C}: \mathrm{n}=5\right)$ 


\subsection{Gbp2 and Hrb1 are involved in translation inhibition of NMD targets}

The NMD pathway prevents the synthesis of aberrant proteins. It promotes mRNA degradation but also translation inhibition. It was shown that in upf1 $\Delta$ cells NMD targets are translated (Dehecq et al., 2018; Kuroha et al., 2009; Muhlrad and Parker, 1999b).

After we had evidence that Gbp2 and Hrb1 are involved in the degradation of NMD targets, we wondered if they also affect the other aspect of NMD - translation inhibition. To analyse the translation of the NMD reporters, we cloned a $M Y C-C B P 80^{P T C}$ and a $M Y C-D B P 2^{P T C}$ reporter and detected the translation products via the MYC tags in Western blot analyses (see Figure $11 \mathrm{~A}, \mathrm{~B}$ and Figure $12 \mathrm{~A}, \mathrm{~B}$ ). Interestingly, the gbp2 $\Delta$ cells showed an increased translation of $M Y C-C B P 80^{P T C}$, while hrb1 $\triangle$ cells showed no difference to wild type. Also, the combination of both knockouts doesn't further increase the reporter translation. This indicates that Gbp2, but not Hrb1, is relevant for the translation inhibition of $M Y C-C B P 80^{P T C}$. Similar to the previous results on the mRNA degradation, it is a partial effect compared to upf1 $\Delta$ cells. To ascertain that this is a translational effect, we also isolated the RNA of the same yeast cultures and quantified the mRNA levels of the NMD reporter. We calculated the relative protein level per mRNA level to get an estimate of the translation rates (see

A

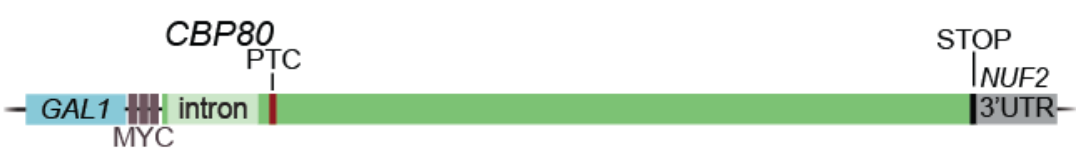

B

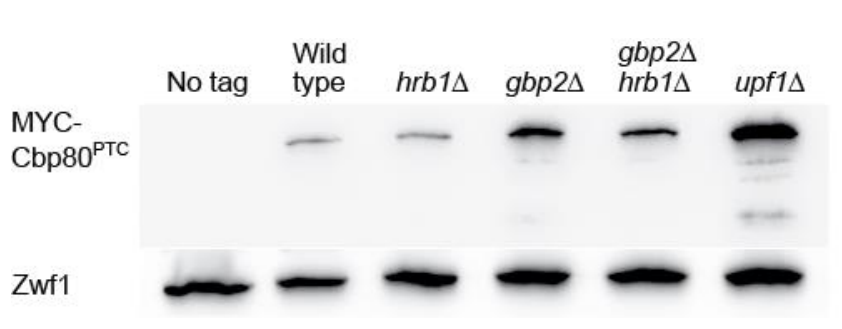

C

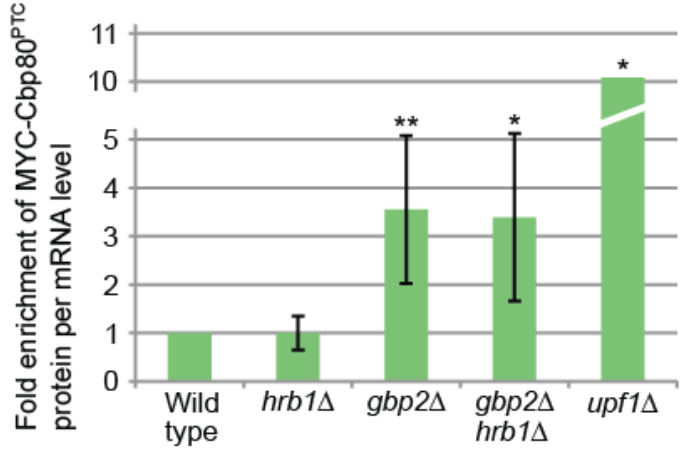

Figure 11: Gbp2 is involved in translation inhibition of $M Y C-C B P 80^{P T C}$.

A Sketch of MYC-CBP80 PTC reporter used in translation analysis. The NMD reporter was induced with galactose for $4 \mathrm{~h}$. Cultures were split, one half was used for Western blot analysis and the other half for RNA isolation. B Western blot of cell lysates from indicated mutants expressing the MYC-CBP80 PTC, and a no tag control. Zwf1 was detected as a loading control. C Quantification of Western blot signals as shown in (B). The MYC signal was normalised to the corresponding Zwf1. The protein levels were related to the MYC$C B P 80^{P T C}$ mRNA level, which was determined by qPCR. The standard deviation of upf1 $\triangle$ cells is 6.5. $(n=5)$ 
Figure $11 \mathrm{C})$. The gbp2 $\Delta$ and gbp2 $\Delta h r b 1 \Delta$ cells showed, on average, a 3.5-fold increased translation rate and the upf1 $\triangle$ strain showed a 10 -fold increased translation rate on average. In contrast to the $M Y C-C B P 80^{P T C}$ reporter, we observed that the $M Y C-D B P 2^{P T C}$ reporter was readily translated in wild type cells upon induction with galactose (data not shown). Here we show the $M Y C-D B P 2^{P T C}$ reporter translation with a low expression level in raffinose medium without galactose induction (see Figure 12). Surprisingly, both the $h r b 1 \Delta$ and gbp2 $\Delta$ strain showed a mildly increased translation of the NMD reporter ( $\sim 2$-fold). The effect did not accumulate in the double mutant, suggesting that both proteins need to be present together to fulfil their function on this reporter. Similar to the $C B P 80$ based reporter, upf1 $\Delta$ cells showed the greatest effect ( 8-fold).

A

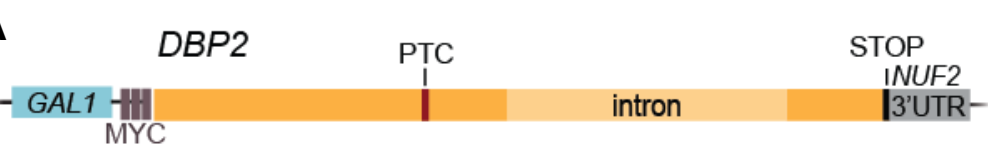

B
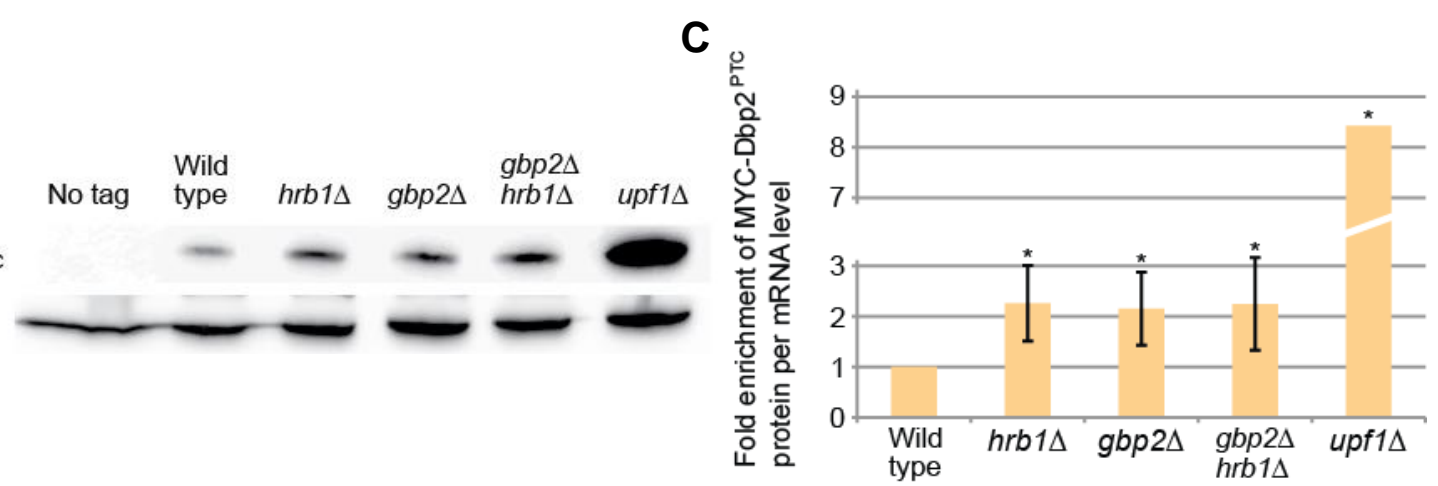

Figure 12: Gbp2 and Hrb1 are involved in translation inhibition of $M Y C-D B P 2^{P T C}$.

A Sketch of MYC-DBP2 ${ }^{\text {PTC }}$ reporter used in translation analysis. The NMD reporter was expressed on a low level in raffinose medium without galactose induction. Cultures were split, one half was used for Western blot analysis and the other half for RNA isolation. B Western blot of cell lysates from indicated mutants expressing the MYC-DBP2PTC, and a no tag control. Zwf1 was detected as a loading control. C Quantification of Western blot signals as shown in (B). The MYC signal was normalised to the corresponding Zwf1 signal. The protein levels were related to the $M Y C-D B P 2^{P T C}$ mRNA level, which was determined by qPCR. The standard deviation of upf1 $\Delta$ cells is 4.1 . $(n=5)$ 


\subsection{The role of Gbp2 and Hrb1 in translation inhibition is Upf1 and PTC dependent}

To verify that Gbp2 and Hrb1 are indeed part of the Upf1 mediated translation inhibition, we compared the NMD reporter translation of the upf1 $\Delta$ strain with the gbp2 $\Delta$ hrb1 $\Delta$ upf1 $\Delta$ strain. Similar to the effect on mRNA degradation, the loss of Gbp2 and Hrb1 has no effect if Upf1 is absent (see Figure 13), indicating further that they function in the same pathway as Upf1. To test whether Gbp2 and Hrb1 are selectively involved in translation inhibition only for NMD targets, we analysed the translation of wild typical CBP80-MYC and DBP2GFP mRNA (see Figure 14, wild typical reporters are shown in Figure 8 A and Figure 9 A). The translation of the wild typical constructs was not increased in the absence of Gbp1 and/or Hrb1. This shows that they are no general translation inhibitors, but rather have a particular function in the NMD pathway.

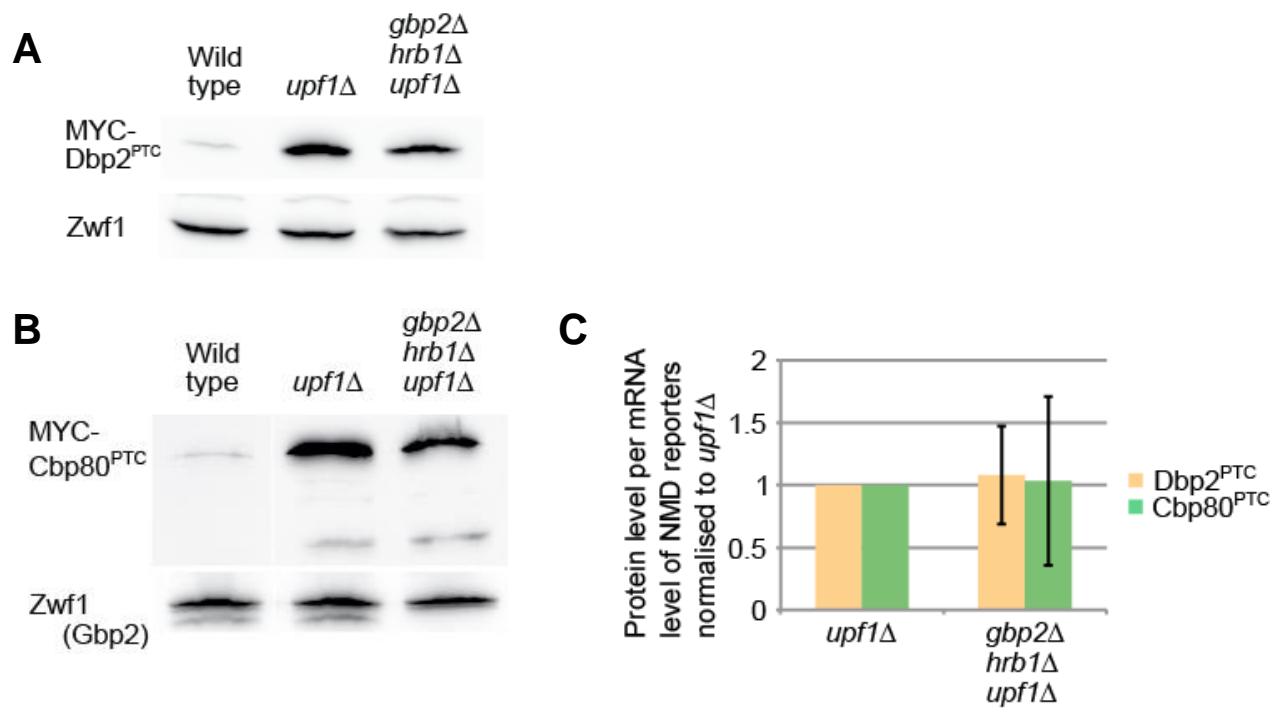

Figure 13: Translation inhibition of Gbp2 and Hrb1 is Upf1 dependent.

Western blot of cell lysates from indicated mutants expressing MYC-DBP2PTC $(\mathbf{A})$ or MYCCBP80 ${ }^{P T C}(\mathbf{B})$. Zwf1 was detected as a loading control. C Quantification of experiments as shown in (A) and (B). The MYC signals were normalised to the corresponding Zwf1 signal. The protein levels were related to the MYC-DBP2 $2^{P T C}$ orMYC-CBP80 ${ }^{P T C}$ mRNA levels. $(\mathrm{n}=4)$ 
A

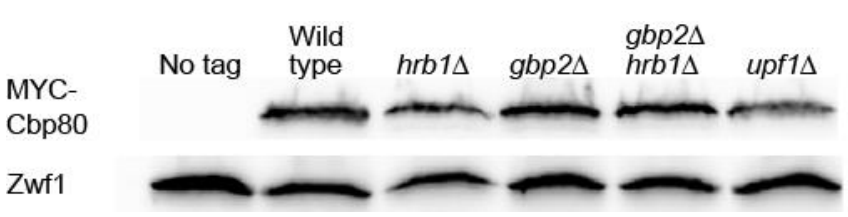

B

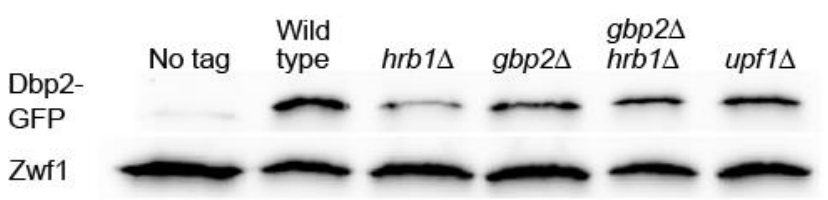

Figure 14: Gbp2 and Hrb1 do not inhibit translation of wild typical CBP80 and DBP2. Western blot showing lysates of indicated strains expressing MYC-CBP80 (A) or DBP2GFP (B) under their own promotors. Zwf1 was detected as a loading control.

\subsection{Gbp2 and Hrb1 are not involved in NMD of PGK1 ${ }^{P T C}$}

As previous results showed no effect on the mRNA level of the non-spliced PGK1 PTC reporter (unpublished data, laboratory of Heike Krebber), we wanted to confirm this finding on the translational level. Thus, we also constructed a MYC-PGK1 ${ }^{P T C}$ reporter (see Figure 15 A). Consistently, we could only see an increased translation in the absence of Upf1 and no visible influence of Gbp2 or Hrb1, suggesting that Gbp2 and Hrb1 may only be part of the NMD machinery on spliced transcripts.

A
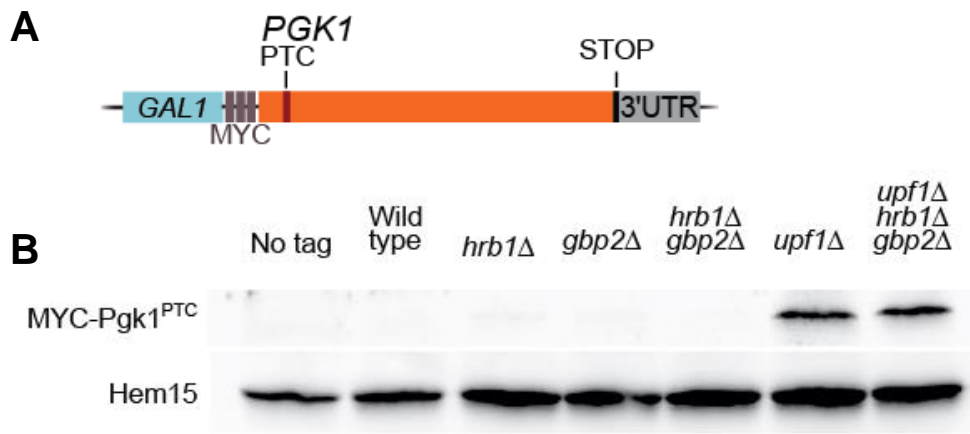

Figure 15: Gbp2 and Hrb1 show no influence on $M Y C-P G K 1^{P T C}$ translation.

A Sketch of MYC-PGK1PTC reporter used in translation analysis. The NMD reporter was induced with galactose for $4 \mathrm{~h}$. B Western blot of cell lysates from indicated mutants expressing the MYC-PGK1PTC reporter and a no tag control. Hem15 was detected as a loading control. 


\subsection{Translation cannot terminate close to the start codon}

In the translation analysis of the NMD reporters, we observed that the translated peptide of the $M Y C-D B P 2^{P T C}$ reporter matched the expected size of truncated Myc-Dbp2 (see Figure 16 A). This suggests that translation terminates at the PTC if it is not recognised as premature. Surprisingly, for the two reporters that have a PTC very close to the start codon, the translation product matches the size of the full-length protein (see Figure 16 B,C). This indicates that, even without Upf1, translation cannot terminate at such a stop codon. Very likely the stop codon is read through, when neither NMD nor regular termination occurs, by

A

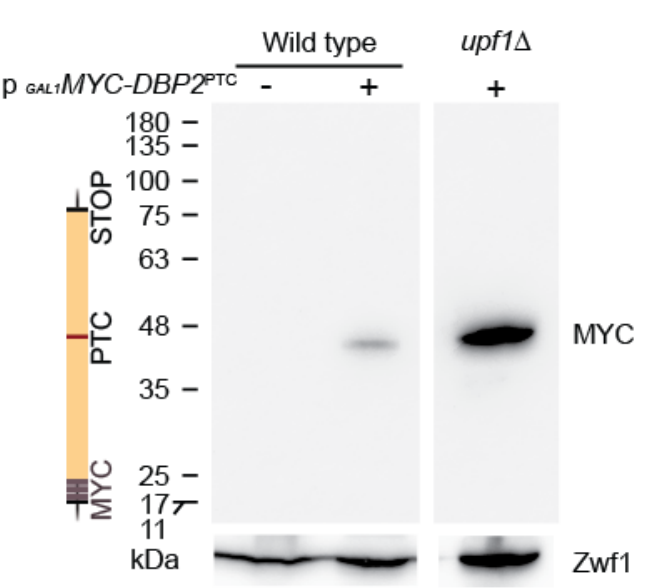

B
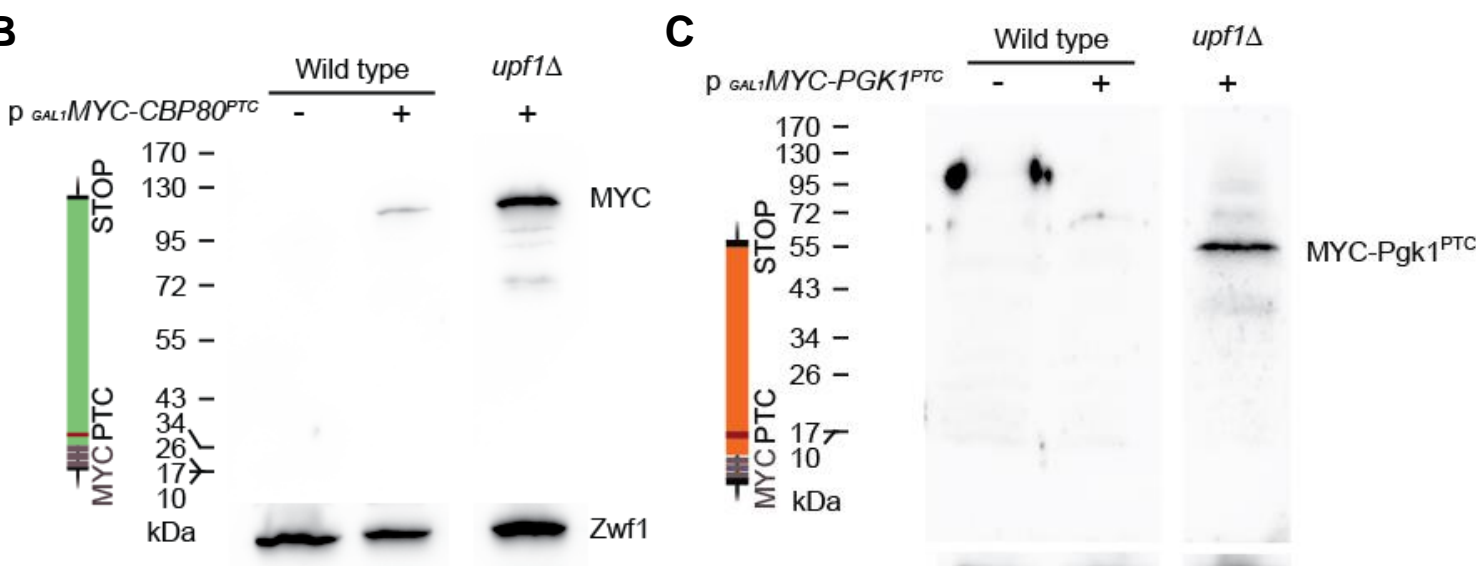

Hem15

Figure 16: Translation cannot terminate at a PTC close to the 5' end.

Western blot analysis as shown in Figure 11, Figure 12 and Figure 15, showing the size of the NMD reporter translation products. Indicated strains were expressing MYC-DBP2PTC (A), MYC-CBP80 PTC $(\mathbf{B})$, or MYC-PGK1 ${ }^{\text {PTC }}(\mathbf{C})$. MYC-Dbp2 ${ }^{\text {PTC }}$ shows the expected size of the truncated protein, when translation terminates at the PTC. Full length MYC-Dbp2 would be expected to run at approx $70 \mathrm{kDa}$. MYC-Cbp80 $0^{\mathrm{PTC}}$ and MYC-Pgk1 ${ }^{\mathrm{PTC}}$ show the sizes of the full length proteins. The truncated peptides through termination at the PTC would be expected to run at approx. $20 \mathrm{kDa}$. 
insertion of a non-matching tRNA, and the ribosome continues until it reaches the normal stop codon.

\subsection{The RGG motif proteins Npl3, Sbp1 and Scd6 are not involved in the translation inhibition of $M Y C-C B P 80^{P T C}$}

We could see that Gbp2 and Hrb1 are involved in the translation inhibition of NMD targets, with Gbp2 appearing to be the relevant factor for the yeast typical $C B P 80^{P T C}$ construct. Both Gbp2 and Hrb1 are RGG motif containing proteins. This group of proteins was already described to be involved in translation initiation inhibition via the RGG motif (Rajyaguru et al., 2012; Segal et al., 2006; Windgassen et al., 2004). It is possible that Gbp2 and Hrb1 perform a similar function on NMD targets. However, it was also described that different proteins of this group can interact via the RGG motif. The three yeast RGG motif proteins

A $\begin{array}{llllll}\text { No Wild } & & & & & \\ \text { tag } & \text { type } & & & & \end{array}$ MYC-Cbp80 $0^{\text {PTC }}$

Zwf1

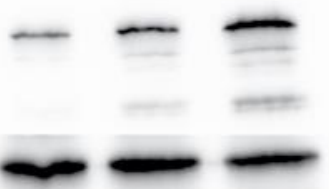

B

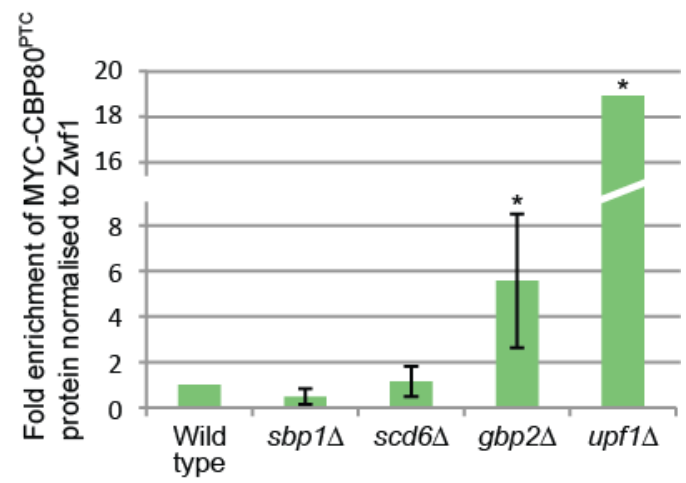

Figure 17: The RGG motif containing translation inhibitors Npl3, Scd6 and Sbp1 show no effect on $M Y C-C B P 80^{P T C}$ translation.

A Western blot showing lysates of indicated mutants expressing MYC-CBP8O ${ }^{P T C}$ by $4 \mathrm{~h}$ galactose induction, and a no tag control. Zwf1 was detected as a loading control. As there is no visible signal in $n p / 3 \Delta$ cells, we verified the NMD reporter RNA by qPCR (data not shown). B Quantification of Western blot signals as shown in (A). The MYC signal was related to the loading control Zwf1. The values represent the steady state protein levels, they were not related to mRNA levels. ( $n=3$, for gbp2 $\Delta$ and upf1 $\Delta \mathrm{n}=5)$ 
Npl3, Scd6 and Sbp1 were described to be general translation inhibitors. To test whether Gbp2 might cooperate with one of these factors in the translation inhibition of MYC$C B P 80^{P T C}$, we looked at the translation of the reporter construct in the knockout strains of either of the three factors (see Figure 17). Yet none of the three knockouts showed an increased translation of the NMD reporter. As these factors don't seem to be involved in NMD, this suggests that Gbp2, as an RGG motif protein, may be involved in the translation inhibition directly. Nevertheless, we cannot exclude that Gbp2 might act via a different factor that acts as a translation inhibitor.

\section{8. elF4E binding to $C B P 80^{P T C}$ is not regulated by the Upf1 pathway}

It was described that elF4E is the cap binding structure in steady state translation (Fortes et al., 2000; Gingras et al., 1999). It is unclear, however, at which step the cap binding structures are exchanged. While NMD is associated with the CBC in higher eukaryotes (Ishigaki et al., 2001; Lejeune et al., 2002; Maquat et al., 2010), the CBC is likely not required for NMD in yeast (Gao et al., 2005). We could see an increased translation of $C B P 80^{P T C}$ in $g b p 2 \triangle$ and upf1 $\triangle$ cells. Therefore, we wondered if this is also reflected on the eIF4E binding to the NMD reporter - assuming that the reporter is CBC bound when it leaves the nucleus, followed by an exchange to elF4E at some step during translation.

UV-crosslinked RNA co-immunoprecipitation (RIP) experiments showed that elF4E has a preferential binding to wild typical CBP80 (see Figure $19 \mathrm{~B}$ ). Surprisingly, this preference is not affected in $g b p 2 \Delta$ and upf $1 \Delta$ cells. The elF4E binding to the NMD reporter is on average slightly lower in upf1 $\Delta$ cells compared to wild type (see Figure $19 \mathrm{C}$ ). This suggests that the translation inhibition might be independent of the cap binding structure and vice versa. Further it indicates that there must be other factors influencing the elF4E binding to the NMD reporter even without Upf1. While on the mRNA level it seemed as though in upf1 $\triangle$ cells the PTC did not affect the CBP80 mRNA (see Figure 8), we saw that during translation the PTC has to be read through. This read through might lead to a lower translation efficiency. And indeed, we found that even in upf1 $\triangle$ cells the translation of $C B P 80^{P T C}$ (while increased compared to wild type cells, see Figure 11) is still considerably lower than the translation of CBP80 mRNA (see Figure 18). It is tempting to speculate that the lowered translation efficiency and the lowered binding of elF4E are connected. In any event, both of these results show, that in upf $1 \triangle$ cells the translational process of $C B P 80^{P T C}$ is not the same as for wild typical CBP80. 
A

Lysates
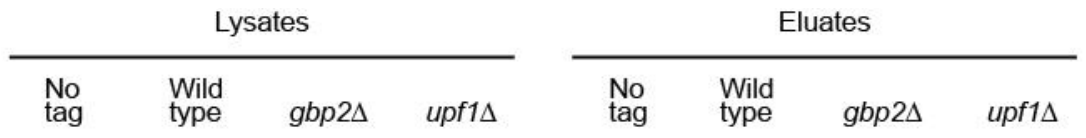

elF4E-GFP

Tdh1

B

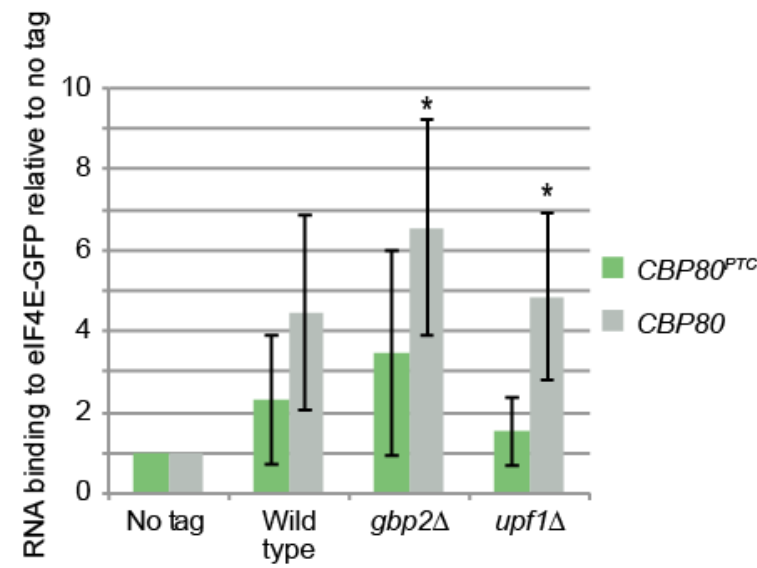

C

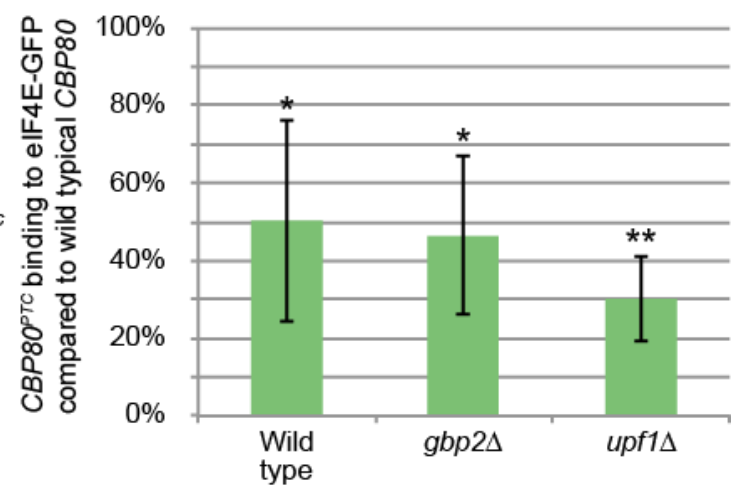

Figure 19: The elF4E binding to $C B P 80^{P T C}$ is not increased in gbp2 $\Delta$ and upf1 $\triangle$ cells.

The CBP8OPTC NMD reporter was induced with galactose for $2 \mathrm{~h}$ in the indicated strains and RNA-protein complexes were crosslinked with UV light. elF4E-GFP RIPs were performed. A Western blots showing the immunoprecipitation of elF4E-GFP. Tdh1 was detected as a control for unspecific binding. B qPCR data from RIPs showing the binding of the CBP80 ${ }^{P T C}$ reporter and endogenous wild typical CBP80 mRNA. The NMD reporter has a NUF2 3' UTR (see Figure 11 A). Revers primers annealing in either CBP80 or NUF2 3' UTR were used to selectively amplify the NMD reporter or wild typical. C qPCR data from (B) with the NMD reporter binding related to the CBP80 mRNA. $(n=4)$

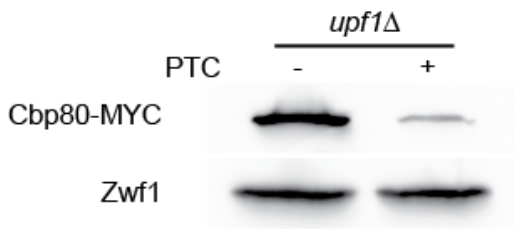

Figure 18: $C B P 80^{P T C}$ is not translated like wild typical $C B P 80$ in upf1 $\triangle$ cells.

Western blot showing lysates of upf1 $\triangle$ cells expressing either CBP80 ${ }^{P T C}-M Y C$ or CBP80MYC under the endogenous promotor (see Figure 8 A). Zwf1 was detected as a loading control. 


\subsection{Gbp2 and Hrb1 mis-localise to the cytoplasm through excessive NMD}

In the previous analysis we could show that both aspects of NMD, cytoplasmic degradation and translation inhibition, are defected in the absence of Gbp2 and Hrb1.

While we have to assume that the effects we saw are indeed cytoplasmic effects (as discussed above), it would, however, still be possible that the absence of Gbp2 and Hrb1 influences other factors in the nucleus, which in turn lead to cytoplasmic effects. To determine whether the two proteins are indeed participating in the cytoplasmic NMD

A

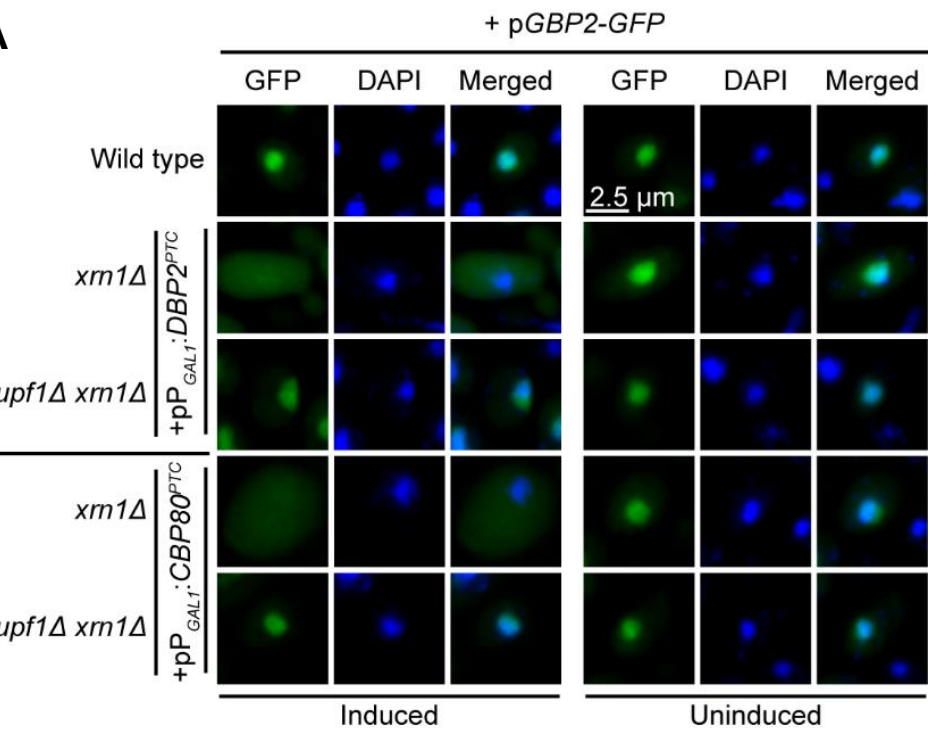

B

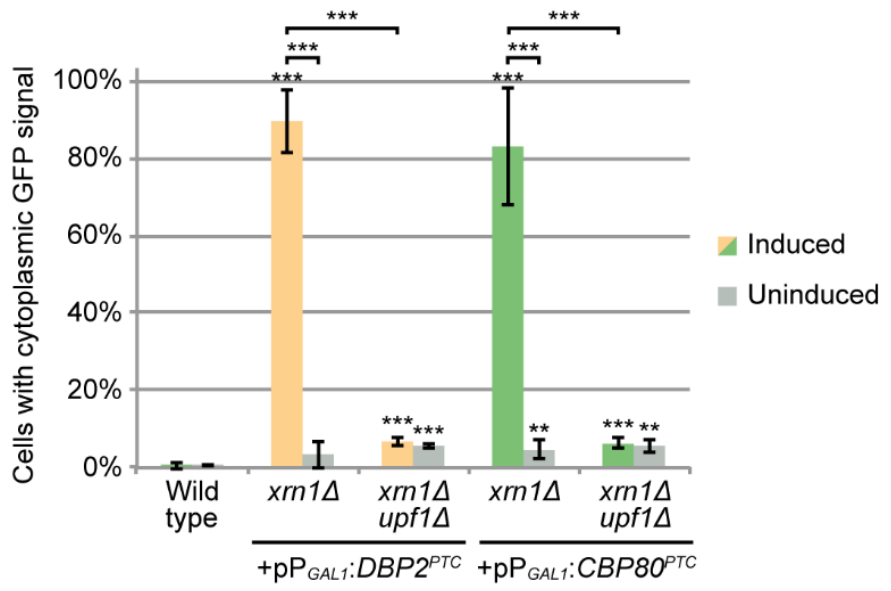

Figure 20: Nuclear re-import of Gbp2 is disturbed by high expression of NMD reporters in the absence of Xrn1.

A Gbp2-GFP fluorescence microscopy signals. Cultures of the indicated mutants containing the indicated plasmids were split in two. One half was induced with galactose for $2 \mathrm{~h}$. DNA was stained with DAPI. B Percentage of cells that showed a mainly cytoplasmic Gbp2-GFP localisation in the analysis of (A). Error bars represent the standard deviation between experiments with 100 - 200 analysed cells per experiment. $(n=3)$ 
pathway themselves, we highly expressed the NMD reporters in the absence of the major degrading enzyme Xrn1 - to see if re-import of Gbp2 and Hrb1 into the nucleus can be disturbed by NMD. Under normal conditions, Gbp2 and Hrb1 are rapidly re-imported into the nucleus and signals of Gbp2-GFP or Hrb1-GFP fusion proteins are almost exclusively nuclear. It was shown previously that mutants of the cytoplasmic kinase Sky1 or the karyopherin Mtr10 lead to a cytoplasmic mis-localisation (Häcker and Krebber, 2004; Windgassen and Krebber, 2003). Interestingly, we could observe that expression of either $C B P 80^{P T C}$ or $D B P 2^{P T C}$ lead to a similar cytoplasmic mis-localisation of both Gbp2 and Hrb1

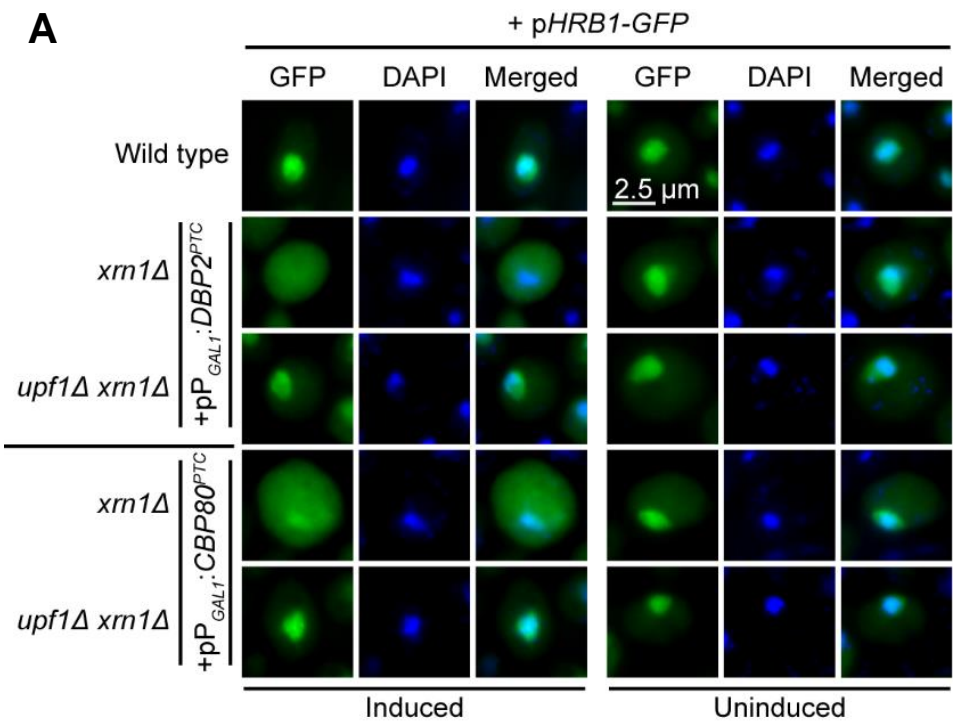

B

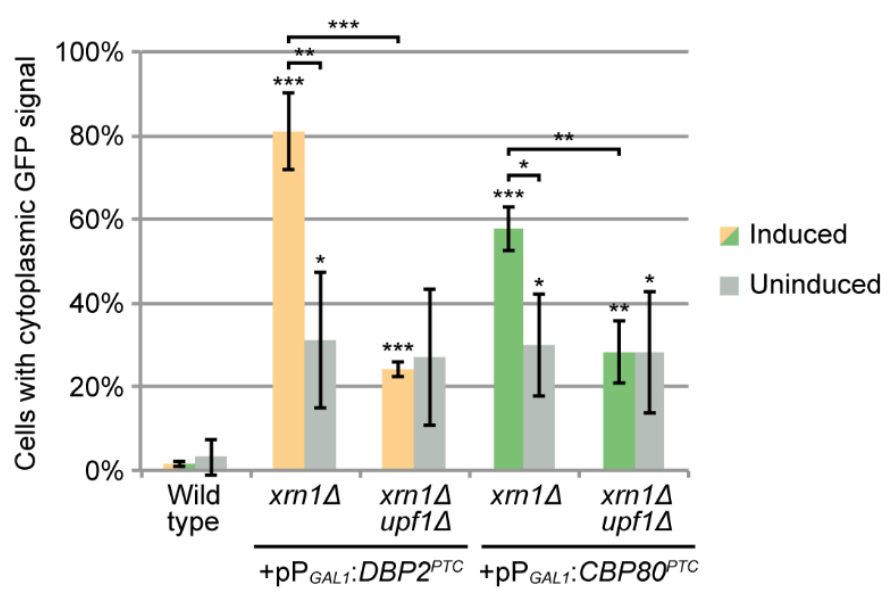

Figure 21: Nuclear re-import of Hrb1 is disturbed by high expression of NMD reporters in the absence of Xrn1.

A Hrb1-GFP fluorescence microscopy signals. Cultures of the indicated mutants containing the indicated plasmids were split in two. One half was induced with galactose for $2 \mathrm{~h}$. DNA was stained with DAPI. B Percentage of cells that showed a mainly cytoplasmic Hrb1-GFP localisation in the analysis of (A). Error bars represent the standard deviation between experiments with 100 - 200 analysed cells per experiment. $(n=3)$ 
(see Figure 20 and Figure 21). This phenotype was completely reverted in the absence of Upf1, suggesting that this is in fact an NMD dependent phenotype. The mis-localisation must be caused by the NMD reporter mRNA and is not an unspecific effect of the translated peptides, because the translation of the NMD reporters is higher in upf1 $\Delta$ cells and these show no effect here. Further, it is surprising that the Hrb1-GFP localisation is disturbed in approx. $30 \%$ of all $x r n 1 \Delta$ cells independent of the NMD reporters or Upf1 (see Figure $21 \mathrm{~B}$ ). This suggests that Hrb1 might also have an NMD independent function associated with the cytoplasmic degradation machinery. On the other hand, the mis-localisation induced by the expression of the NMD reporters is less pronounced for Hrb1-GFP. Especially for the yeast typical $C B P 80^{P T C}$ reporter. Approx. $60 \%$ of the cells show a mis-localised Hrb1-GFP signal (see Figure 21 B; 80 \% for Gbp2-GFP, see Figure 20 B).

The NMD dependent mis-localisation of Gbp2 and Hrb1 is a first indication that the two proteins are directly involved in the NMD pathway. This finding is supported by physical interactions of both proteins with Upf1, Upf2 and Upf3 and split-GFP experiments indicating that Gbp2 and Upf1 are in close proximity in the cell upon induction of the $C B P 80^{P T C}$ reporter (unpublished data, laboratory of Heike Krebber, for split-GFP see Ghosh et al., 2000; Magliery et al., 2005).

\subsection{Gbp2 and Hrb1 interact with the cytoplasmic degradation machinery}

We could see that Gbp2 and Hrb1 are involved in nonsense mediated decay. Further, we addressed how they might be involved in the NMD mechanism. Upf1 presumably interacts with all mRNAs - mostly in the 3' UTR because it is removed by the translating ribosome (Hurt et al., 2013; Kurosaki et al., 2019). However, Upf1 preferentially binds NMD targets (Johansson et al., 2007). Upon initiation of NMD, Upf1 is presumably recruited to the prematurely terminating ribosome (Kervestin and Jacobson, 2012). The binding of Upf1 to NMD targets is dependent on factors that promote the NMD initiation. The mechanism of loading Upf1 to ribosomes that terminate prematurely is not understood. However, in human cells it was observed that a knockdown of UPF2, UPF3 or elF4A3 (a core component of the EJC) reduces the binding of Upf1 to NMD reporters (Kurosaki et al., 2014). Either UPF1 is not efficiently loaded to the site of premature termination or is not stabilised there. As a consequence, NMD is not initiated correctly. RNA co-immunoprecipitation experiments with the $C B P 80^{P T C}$ reporter in yeast cells showed that the Upf1 binding is strongly reduced in upf2 $\Delta$ cells but unaffected in gbp2 $\Delta$ hrb1 $\Delta$ cells (unpublished data, laboratory of Heike Krebber). This led us to believe that Gbp2 and Hrb1 do not affect the initial step of NMD, but rather facilitate the downstream effects. To test the hypothesis that Gbp2 and Hrb1 might help in mediating the mRNA degradation after NMD is initiated by Upf1, we tested if 
Gbp2 and Hrb1 physically interact with the cytoplasmic degradation machinery. We immunoprecipitated Dcp1-GFP of the mainly cytoplasmic decapping holoenzyme, a factor of the 5'-3' degradation machinery, and Ski2-GFP of the Ski complex, a factor of the cytoplasmic 3'-5' degradation machinery. Both IP experiments showed a co-precipitation of Gbp2 and Hrb1 with and without addition of RNase (see Figure 22 A and B). Initial experiments showed that the interaction between Dcp1 and Gbp2 was lost through RNase treatment (data not shown). Here we show, that with formaldehyde crosslinking both Hrb1 and Gbp2 co-precipitate with Dcp1 after RNase treatment, even though Dcp1-GFP shows a poor precipitation after the formaldehyde treatment. This suggests that Gbp2 is in a complex with Dcp1 but requires RNA binding, presumably for the right conformation. Likewise, the Ski2-GFP IP showed a diminished interaction with Gbp2 upon RNase treatment. This might also be a consequence of Gbp2 requiring RNA binding and/or reflect that multiple Gbp2 proteins might interact with the same mRNP as Ski2, but not all of them are in the same protein complexes.
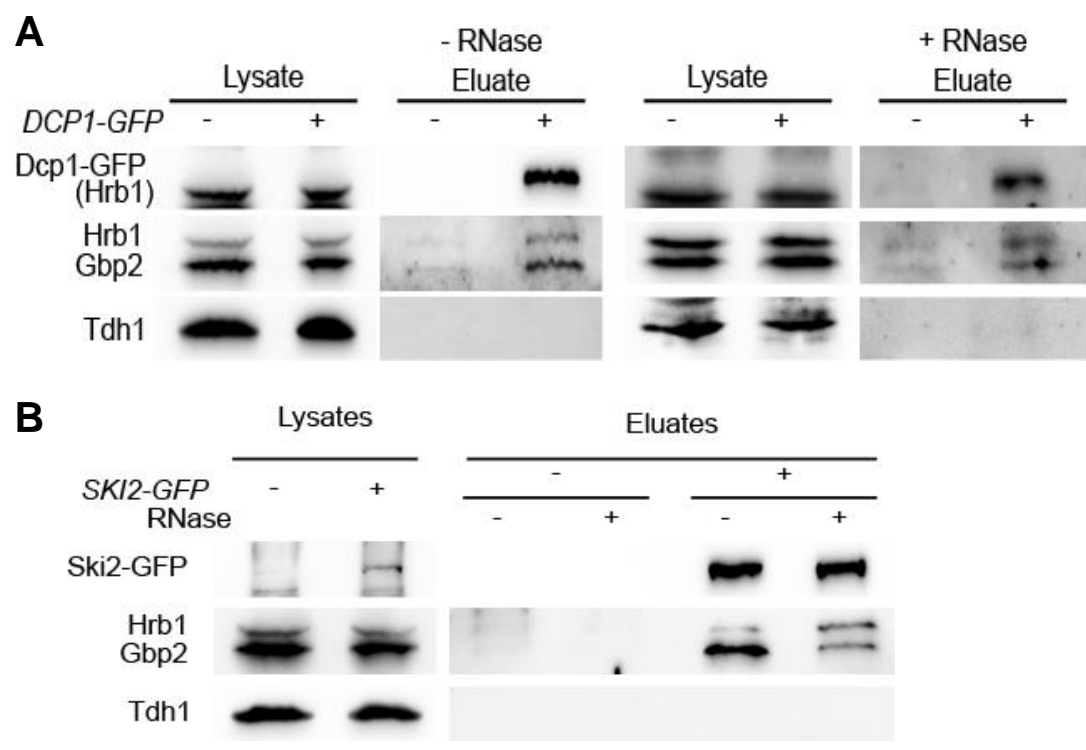

Figure 22: Gbp2 and Hrb1 physically interact with the cytoplasmic degradation machinery.

A Immunoprecipitation of Dcp1-GFP showing the co-precipitation of Gbp2 and Hrb1. The RNase treated IP was performed in a separate experiment, in which proteins were crosslinked with $1 \%$ formaldehyde. Tdh1 was detected as a control for unspecific binding. B Immunoprecipitation of Ski2-GFP showing the co-precipitation of Gbp2 and Hrb1. Tdh1 was detected as a control for unspecific binding. Note that non-RNase treated samples were washed 6 times, to remove unspecific interactions with the GFP selector beads, while RNase treated samples were washed 4 times. Hence, the Hrb1 co-precipitation appears weaker without RNase treatment. 


\subsection{Hrb1 promotes the recruitment of Dcp1 to NMD targets}

Gbp2 and Hrb1 are known to be required for recruiting the nuclear degradation machinery to faulty mRNAs (Hackmann et al., 2014). After we discovered that Gbp2 and Hrb1 also interact with the cytoplasmic degradation machinery, we tested whether they are similarly involved in recruiting the cytoplasmic degradation machinery to NMD targets. Immunoprecipitation of Dcp1-GFP revealed that the co-precipitation of Upf1-HA is reduced in gbp2 $\Delta r b 1 \Delta$ cells (see Figure 23). This was confirmed by UV-crosslinked RNA coimmunoprecipitation (RIP) of Dcp1-GFP with the $M Y C-C B P 80^{P T C} \mathrm{NMD}$ reporter. The Dcp1 bound $C B P 80^{P T C}$ RNA was normalised to the endogenous, wild typical CBP80 RNA to exclude NMD independent effects and to negate errors of the experimental procedure. The wild typical mRNA showed, on average, no altered binding to Dcp1 in the mutant strains compared to wild type (see Figure $24 \mathrm{~B}$ ). The RIP results show that the Dcp1 binding to the NMD reporter is reduced to approx. $40 \%$ in upf1 $\triangle$ cells (see Figure $24 \mathrm{C}$ ). This represents the level of the wild typical CBP80 mRNA (see Figure $24 \mathrm{~B}$ ). As expected, the gbp2 $\triangle$ hrb1 $\triangle$ cells showed a partial effect (a reduction to approx. $60 \%$ ) compared to the upf1 $\Delta$ strain. Interestingly, the RIP shows that Gbp2 is dispensable for the Dcp1 recruitment, while hrb1 $\Delta$ shows the same effect as gbp2 $\Delta$ hrb1 . Hence, Hrb1 seems to promote the Dcp1 recruitment without Gbp2.
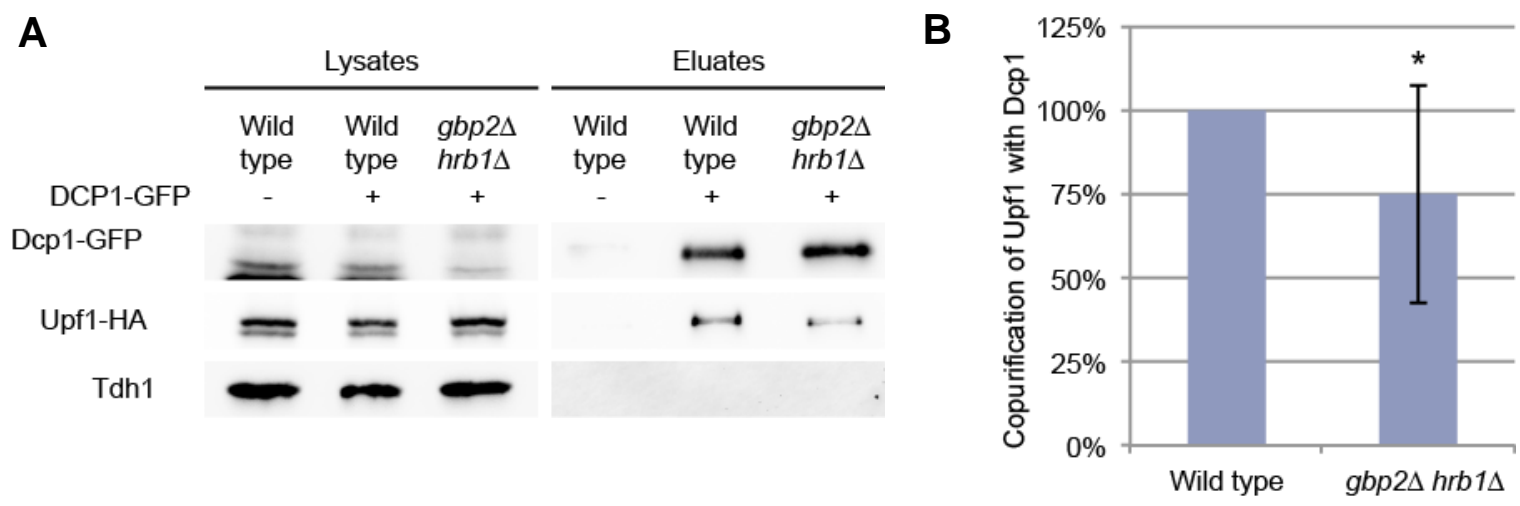

Figure 23: The interaction between Dcp1 and Upf1 is decreased in gbp2 $\Delta$ hrb1 $\Delta$ cells. A Western blot of Dcp1-GFP immunoprecipitation in the indicated strains showing the coprecipitation of Upf1-HA. The samples were treated with RNase. Tdh1 was detected as a control for unspecific binding. B Quantification of Upf1 co-precipitation with Dcp1-GFP as shown in (A). The Upf1-HA signals were normalised to the corresponding Dcp1-GFP pulldown signals. $(\mathrm{n}=8)$ 
A
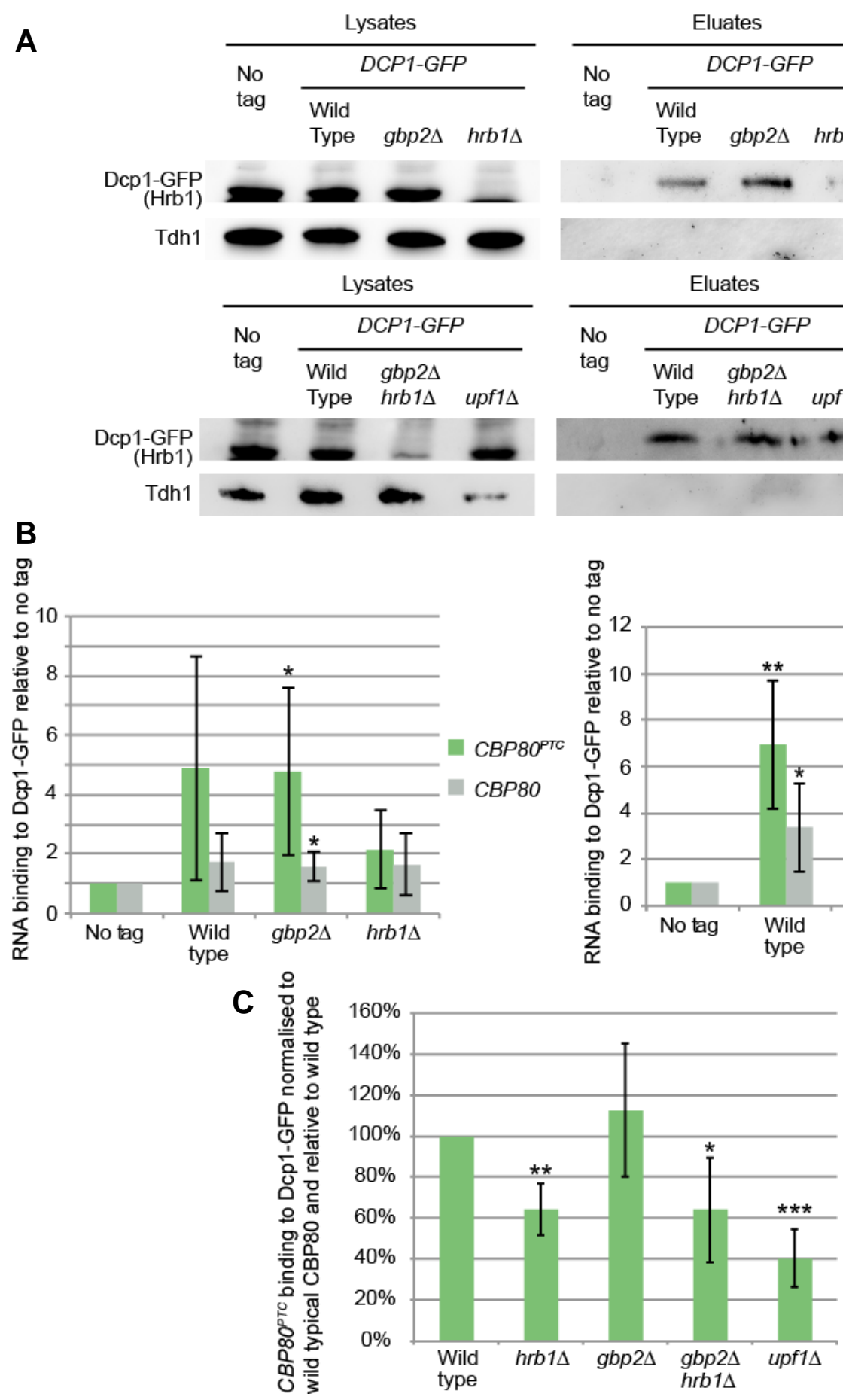

Figure 24: Hrb1 promotes the binding of Dcp1 to the $C B P 80^{P T C}$ NMD reporter.

The MYC-CBP80 ${ }^{P T C}$ NMD reporter was induced with galactose for $2 \mathrm{~h}$ in the indicated strains and RNA-protein complexes were crosslinked with UV light. Dcp1-GFP RNA coimmunoprecipitations (RIPs) were performed. A Western blots showing the immunoprecipitation of Dcp1-GFP. Tdh1 was detected as a control for unspecific binding. B qPCR data from RIPs showing the binding of the CBP80PTC reporter to Dcp1-GFP. Endogenous, wild typical CBP80 mRNA was detected as a control. C qPCR data from (B) with the NMD reporter levels normalised to the CBP80 mRNA and related to wild type. $(n=5$, for $h r b 1 \Delta \mathrm{n}=6$ ) 


\subsection{Gbp2 and Hrb1 are not required to recruit Xrn1 to the $C B P 80^{P T C}$ RNA}

As Gbp2 and Hrb1 are bound to mRNAs during translation, it seems reasonable that they are involved in early steps of NMD. However, previous results showed that Gbp2 and Hrb1

A

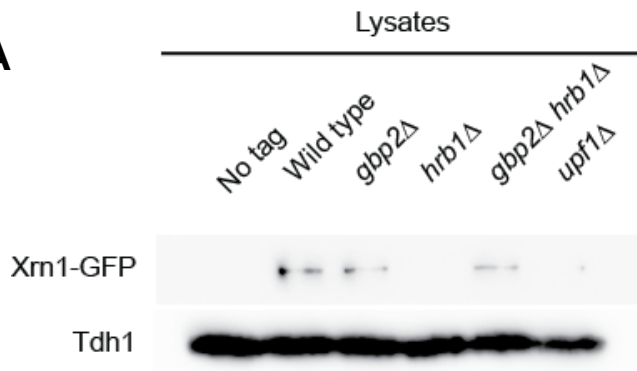

B

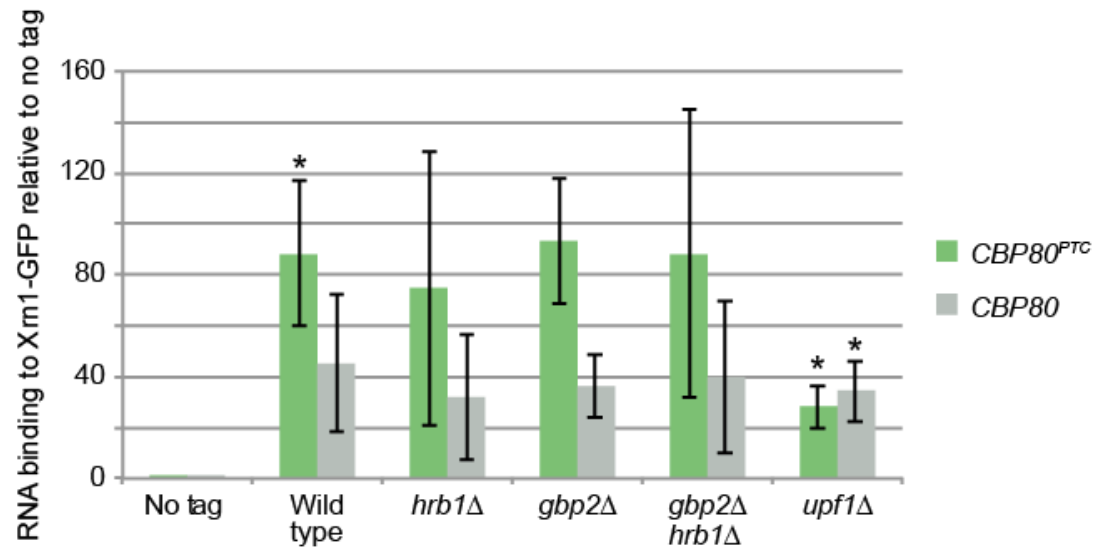

C

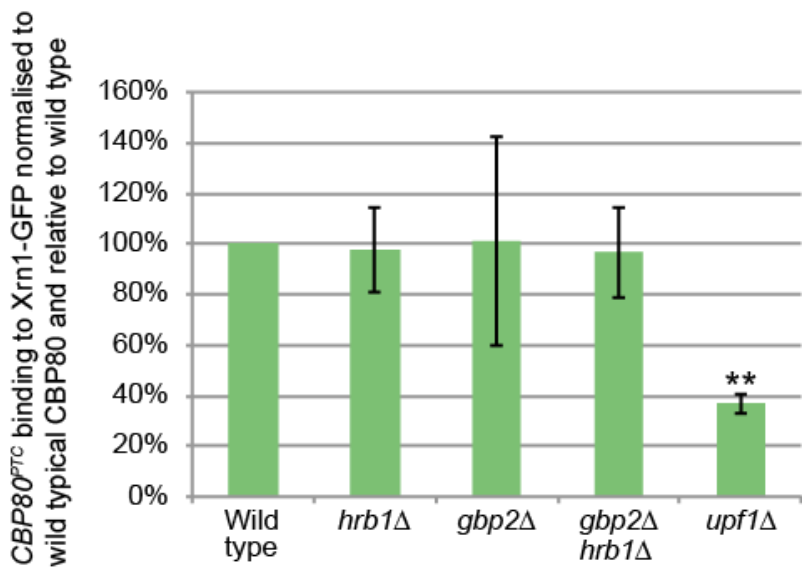

Figure 25: $C B P 80^{P T C}$ NMD reporter binding to Xrn1-GFP is unaltered in gbp2 $\Delta$ hrb1 $\Delta$ cells.

The CBP80 PTC NMD reporter was induced with galactose for $2 \mathrm{~h}$ in the indicated strains and RNA-protein complexes were crosslinked with UV light. Xrn1-GFP RIPs were performed. A Western blots showing the immunoprecipitation of Xrn1-GFP. Tdh1 was detected as a control for unspecific binding. B qPCR data from RIPs, showing the binding of the CBP80 PTC reporter and wild typical CBP80 mRNA to Xrn1-GFP. C qPCR data from (B) with the NMD reporter levels normalised to the CBP80 mRNA and related to wild type. $(n=3$, for gbp2 $\Delta$ $n=2)$ 
have an RNase resistant interaction with the degrading enzyme Xrn1 (unpublished data, laboratory of Heike Krebber). We tested if Gbp2 and/or Hrb1 might also affect the recruitment of Xrn1. However, UV crosslinked RIP experiments showed no reduced association of the CBP80 ${ }^{P T C}$ reporter RNA to Xrn1-GFP in the absence of Gbp2, Hrb1 or both (see Figure 25). As expected, the Xrn1 association to the reporter is reduced in upf1 $\triangle$ cells to level of the wild typical CBP80 mRNA.

\subsection{Gbp2 and Hrb1 interact with each other and themselves}

Gbp2 and Hrb1 share a high sequence homology and were already described to function in the same pathway in the nucleus. Here we found that in the cytoplasm they show different roles in the same pathway. We expected that Gbp2 and Hrb1 are present in similar protein

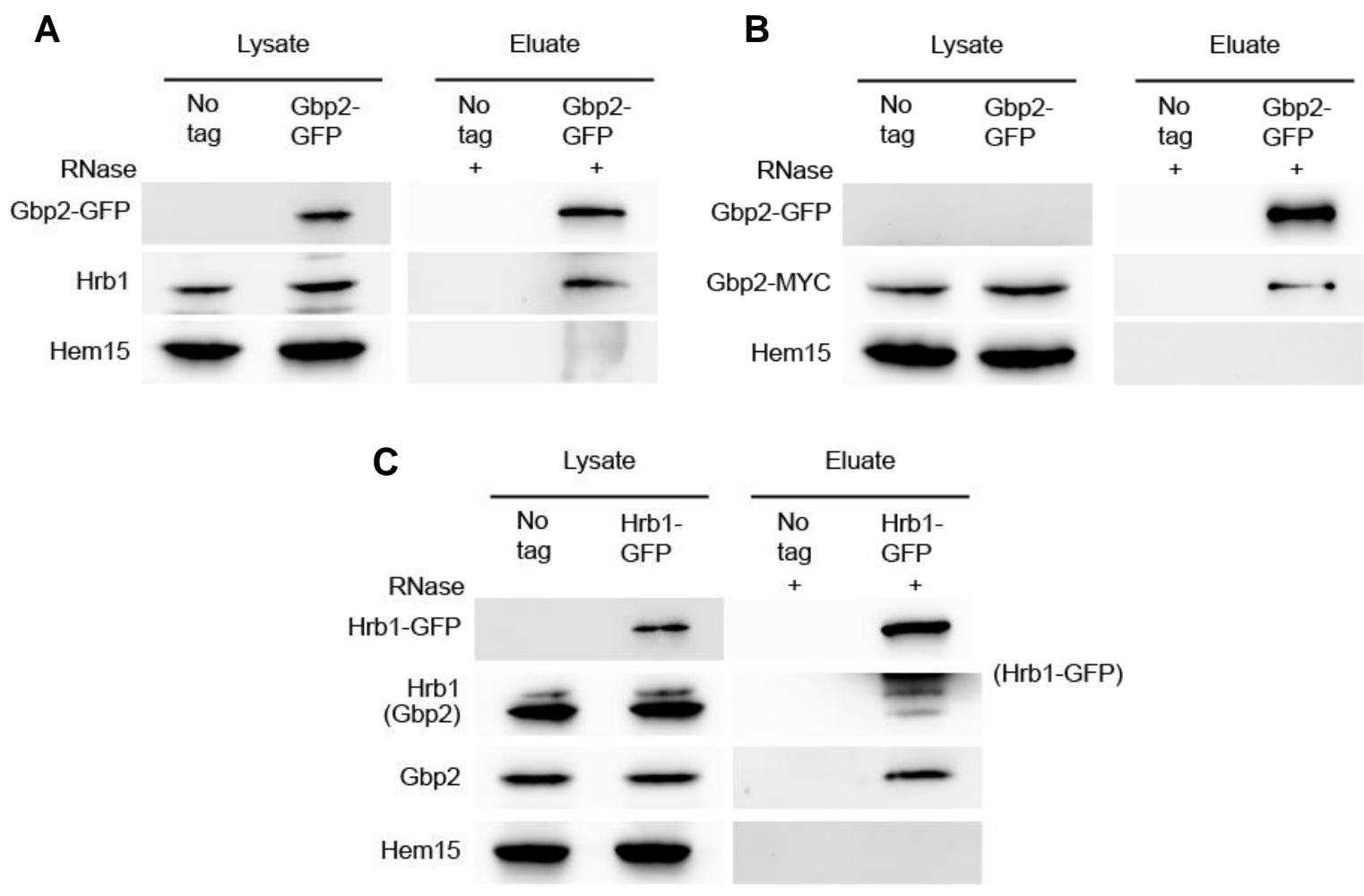

Figure 26: Gbp2 and Hrb1 show RNase resistant interactions with each other and themselves.

A IP of Gbp2-GFP showing the interaction with endogenous Hrb1. B IP of Gbp2-GFP showing the interaction with Gbp2-MYC. C IP of Hrb1-GFP showing the interaction with endogenous Gbp2 and Hrb1. In (A), (B) and (C) Hem15 was detected as a control for unspecific binding to the beads. MYC- or GFP-tagged Gbp2 or Hrb1 were plasmid encoded and detected with antibodies for the respective tag. Untagged Gbp2 or Hrb1 were detected with direct antibodies. 
complexes. Gbp2 and Hrb1 show a physical interaction that is RNase resistant in Gbp2GFP or Hrb1-GFP IPs (see Figure 26 A and C). Interestingly, both proteins also showed RNase resistant interactions with themselves. We used Gbp2-MYC to test the interaction with Gbp2-GFP (see Figure 26 B). We could, however, not use Hrb1-MYC in the same manner. The expression level appears to be too low and Hrb1-MYC showed only very poor signals with the MYC antibody (data not shown). Still, we could detect the endogenous, untagged Hrb1 co-precipitated with plasmid encoded Hrb1-GFP (see Figure 26 B). The IP results indicate that some complexes contain several Gbp2 and Hrb1 molecules at the same time.

\subsection{Gbp2 and Hrb1 have a stabilising effect on normal mRNAs}

In our studies we observed that the mRNA levels of wild typical CBP80 and DBP2 are lowered in gbp2 $h r b 1 \Delta$ cells (see Figure $27 \mathrm{~A}$ and B). This effect appears to be independent of a PTC and Upf1. The effect is especially pronounced for CBP80, which is

A

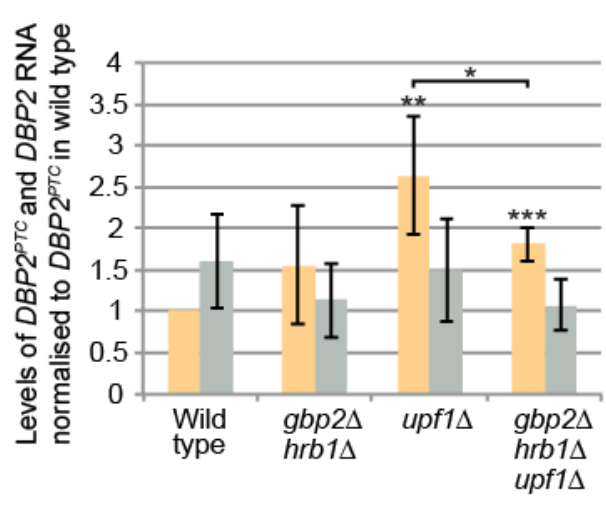

B

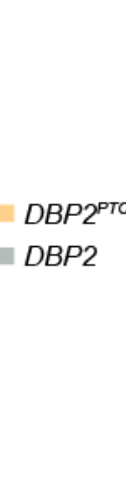

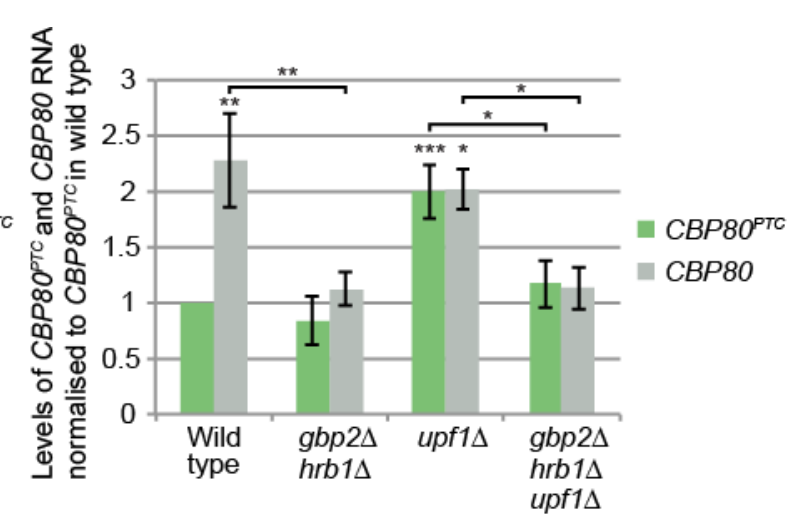

C

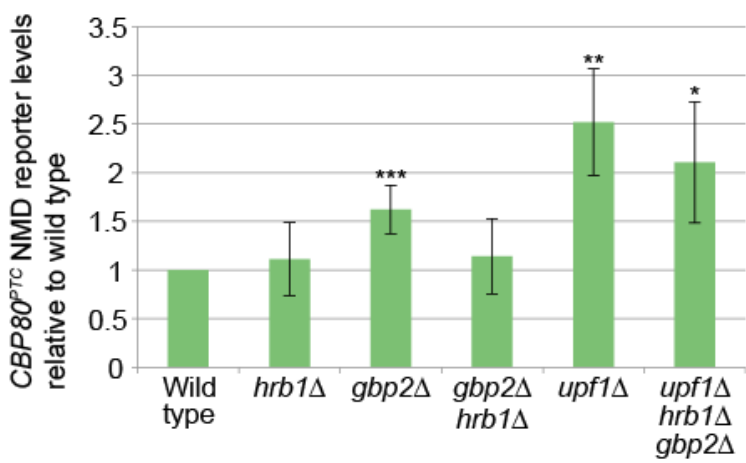

Figure 27: Loss of Gbp2 and Hrb1 leads to reduced mRNA levels.

Steady state RNA levels of wild typical and PTC containing CBP80 (A) or DBP2 (B), as shown in Figure 8 B and Figure 9 B, respectively. Here, all values were normalised to the NMD reporter containing wild type strain. C Steady state RNA levels of galactose induced MYC-CBP80 ${ }^{P T C}$ in the performed translation analysis see Figure 11 and Figure 13. $(n=6)$ 
reduced $\sim 50 \%$. In the translation analysis of $C B P 80^{P T C}$ we observed that the total level of translated protein is reduced in $g b p 2 \Delta h r b 1 \Delta$ cells compared to the gbp2 $\Delta$ single mutant (see Figure $11 \mathrm{~B}$ ). The same is true for the upf1 $\mathrm{gbp} 2 \Delta$ hrb1 $\Delta$ strain compared to the upf1 $\Delta$ mutant (see Figure $13 \mathrm{~B}$ ). However, the translation rate is, on average, the same (see Figure $11 \mathrm{C}$ and Figure $13 \mathrm{C}$ ). The reduction of protein level is reflected by an equally reduced mRNA level (see Figure $27 \mathrm{C}$ ). As Gbp2 and Hrb1 are known to be involved in nuclear quality control, it is reasonable to assume that the reduced RNA level is a nuclear effect, which lowers the effective transcription rate. Surprisingly, when we tested the stability of the $C B P 80^{P T C}$ reporter, we found that the half-life of the reporter seems to reflect the steady state RNA level (see Figure 28 A). This indicates that the loss of Gbp2 and Hrb1 lowers the mRNA stability as seen in the reduced half-life in upf1 $\mathrm{gbp} 2 \Delta$ hrb1 $\Delta$ compared to upf1 1 .

When we tested the steady state levels of several mRNAs in gbp2 $\Delta$ hrb1 $\Delta$ compared to wild type, we were surprised that mRNAs that do not undergo splicing are also affected (see Figure $28 \mathrm{~B}$ ). For most mRNAs, however, the effect appears to be relatively mild, the steady state levels were reduced $10-30 \%$ with the majority of mRNAs showing a reduction of approx. $20 \%$. As expected, the mitochondrial $21 \mathrm{~S}$ rRNA is not affected by this. Further, we were surprised to find the reduced RNA levels were reverted in the absence of Xrn1, indicating that this is a cytoplasmic effect. Apart from their involvement in NMD, Gbp2 and Hrb1 appear to have a generally stabilising effect on mRNAs in the cytoplasm. 
A

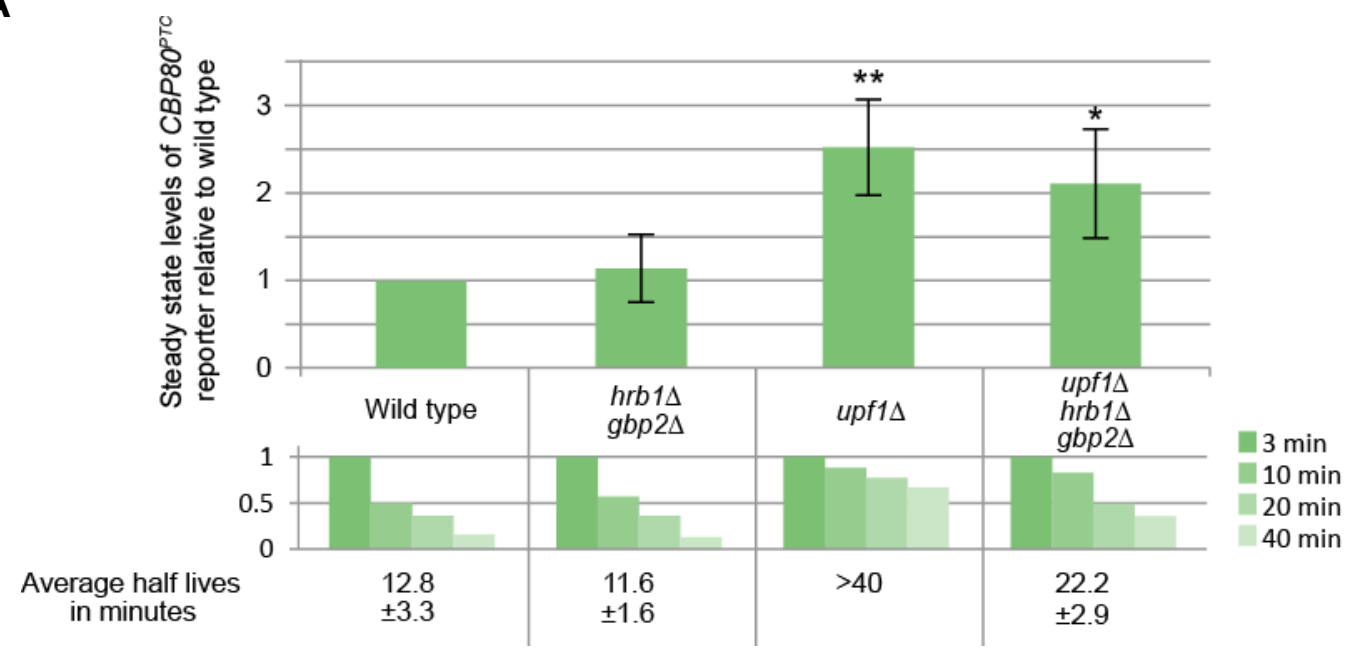

B

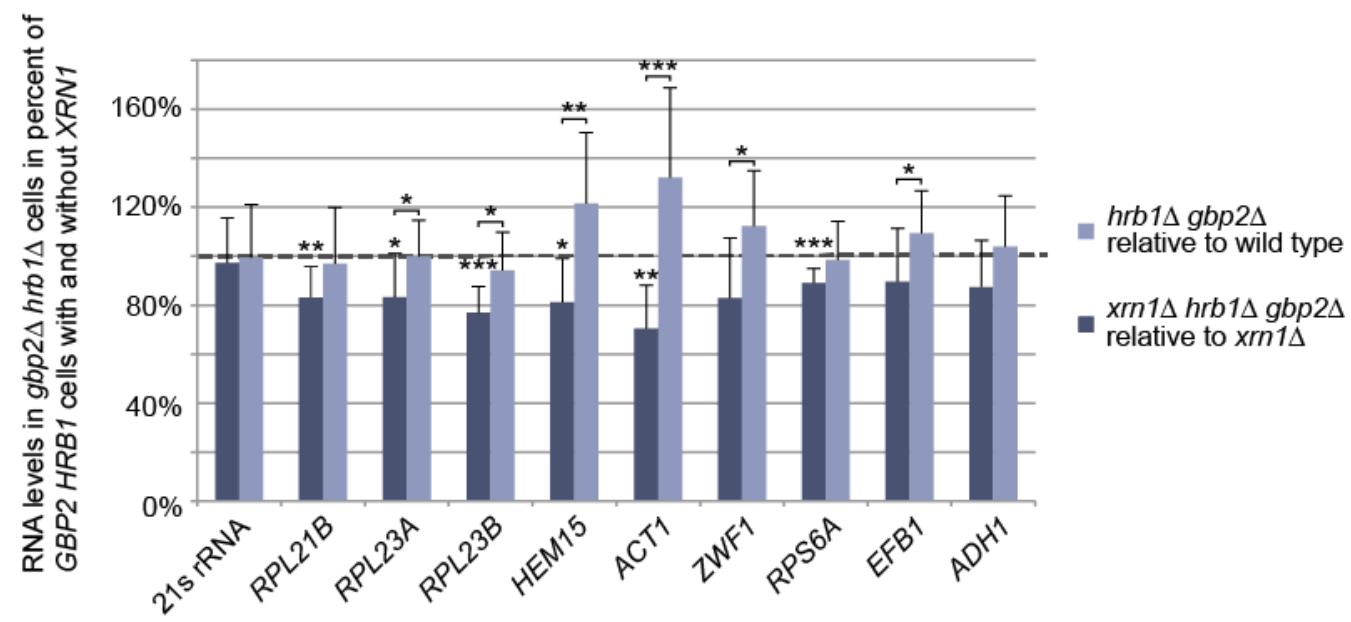

Figure 28: The absence of Gbp2 and Hrb1 causes an Xrn1 dependent destabilisation of mRNAs.

A Comparison of the steady state level of MYC-CBP8OPTC after $4 \mathrm{~h}$ of galactose induction (as shown in Figure $27 \mathrm{~B}$ ), and the MYC-CBP80 ${ }^{P T C}$ half-life. To measure the half-life, the NMD reporter was induced with galactose for $30 \mathrm{~min}$ and the transcription was stopped by addition of glucose. Yeast cells were harvested after 3, 10, 20 and 40 min and used for RNA isolation. ( $n=3)$ B Steady state levels of several mRNAs in the absence gbp2 $\Delta$ hrb $1 \Delta$. The levels are shown in percent of the wild typical level. The same comparison was performed in the absence of Xrn1. HEM15, ZWF1 and ADH1 have no intron and are, therefore, not spliced. $(n=8)$ 


\section{Discussion}

\subsection{Gbp2 and Hrb1 are part of cytoplasmic quality control}

In our studies we found evidence that Gbp2 and Hrb1 are involved in NMD and we could start to unravel the mechanism of their function.

We could see that Hsp104-RFP forms visible fluorescent foci (see Figure 7, page 62) as described in Jamar et al., 2018. It was described that this is an effect of protein aggregation in mutants of cytoplasmic quality control. The finding that $\sim 16 \%$ of the gbp2 $\Delta$ hrb $1 \Delta$ cells showed Hsp104-RFP foci generally indicates that cytoplasmic quality control is defective this strain. In our upf1 $\Delta$ control strain approx. $17 \%$ of the cells showed these foci, which matches the original finding. In our studies it was, however, less consistent between experiments. In contrast to the original publication, we used a temperature shift to $37^{\circ} \mathrm{C}$ for one hour. In initial experiments, we found that the Hsp104-RFP foci were poorly visible without the temperature shift, however, we used $25^{\circ} \mathrm{C}$ as normal yeast growth conditions, while yeast cells were grown at $30{ }^{\circ} \mathrm{C}$ in the original publication. Also, here we used the BY4741/BY4742 strain backgrounds, while it was originally shown in a 74D-694 derived background. Both of these factors might cause the difference. In our wild type strain $5 \%$ of the cells showed Hsp104-RFP foci, which is almost comparable to the reported $3 \%$. The slightly increased phenotype in wild type cells might likewise be a consequence of the raised temperature.

As $m / p 1 \Delta$ cells showed no difference to wild type, we assume that mutants, which exclusively affect nuclear quality control, cause no increased protein aggregation. This supports the hypothesis that Gbp2 and Hrb1 have an additional function in the cytoplasm. While a defective nuclear quality control might lead to mistranslation through the export of aberrant mRNAs, this defect is probably compensated if the cytoplasmic quality control is functional. But we cannot entirely exclude the possibility that other mutants of the nuclear quality control might cause Hsp104-RFP aggregation. Mlp1 is, however, relevant for retaining unspliced pre-mRNA in the nucleus - similar to Gbp2 and Hrb1 (Hackmann et al., 2014). If this leakage alone would cause Hsp104-RFP foci, we should see it in the m/p1A strain. Since this was not the case, it suggests that cytoplasmic quality control must be defective in gbp2 $\Delta$ hrb1 $1 \Delta$ cells. However, if cytoplasmic quality control is defective, it is possible that the increased leakage of unspliced pre-mRNA might partially contribute to the Hsp104 aggregation, because a defective cytoplasmic quality control might not be able to compensate the leakage. This might explain, why the effect of gbp2 $\Delta$ hrb1 $\Delta$ was comparable to upf $1 \Delta$, although in all following experiments upf1 $1 \Delta$ cells always showed stronger effects than $g b p 2 \Delta h r b 1 \Delta$ cells. Similarly, in this experiment the effect of the upf1 $\Delta$ gbp2 $\Delta$ hrb1 $\Delta$ triple deletion was, on average, mildly stronger than that in upf1 $\Delta$ alone. 
These observations might also be caused by a defect in the other cytoplasmic quality control pathways, given that in the original publication NGD and NSD mutants only had a mild effect in this assay ( $8 \%$ of cells). It is generally to say that we don't know how different mutants are quantitatively comparable in this assay. It would be interesting to test whether Gbp2 and Hrb1 might also affect NGD and NSD. Similar to NMD reporters, there are specific reporter constructs for analysing these decay pathways (Tsuboi et al., 2012). As initial experiments, such constructs could be used in gbp2 $\Delta$ hrb1 $1 \Delta$ cells to test if the decay is affected. It might, however, be relevant to clone intron containing reporters if these are the main targets of the Gbp2/Hrb1 function.

\subsection{Degradation of NMD targets is defective in $g b p 2 \Delta$ hrb1 $\Delta$ cells}

In our analysis of PTC containing NMD reporters, we could see that the destabilising effect of the PTC is reduced in the gbp2 $\Delta$ hrb1 $\Delta$ strain (see Figure 8, page 64 and Figure 9, page 65). As discussed in section 4.2, we have to assume that this is a co-translational effect, because the PTC is frame dependent. The CBP80 ${ }^{P T C}$ reporter showed that NMD on this reporter was reduced approx. by half (the mRNA level was reduced $\sim 20 \%$ by the PTC in gbp2 $\Delta h r b 1 \Delta$ and $\sim 55 \%$ in wild type). The reporter RNA level was unaffected by the PTC if Upf1 was missing. This suggests that without Upf1 the NMD reporter is not recognised as aberrant and the cell does not differentiate between $C B P 80^{P T C}$ and (wild typical) CBP80. However, we found out later that the translational processes of the NMD reporter must be different from the wild typical mRNA, also in the upf1 $\triangle$ strain (see 4.8 and 5.4 ). The other NMD reporter, $D B P 2^{P T C}$, showed that the PTC causes a destabilisation in wild type cells but a stabilisation in the NMD mutants (see Figure 9, page 65). As we analysed steady state RNA levels, this might be an artefact if the transcription of the wild typical control reporter is defective. However, both constructs were sequenced and are identical with exception of the PTC. However, the wild typical reporter likely produces a functional protein. It is not unlikely that a feedback loop regulates the transcription rate to maintain the cellular Dbp2 protein level. In that case, the endogenous $D B P 2$ gene and the plasmid encoded reportergene might each transcribe $D B P 2$ at a reduced rate.

The PTC appears to cause a mild reduction of the DBP2 mRNA level in wild type cells (reduced $\sim 35 \%$ ). This was expected because NMD reporters with a PTC closer to the regular stop codon are described to be worse NMD substrates (Peltz et al., 1993), indicating that these results may indeed reflect the PTC effect on $D B P 2$. It is possible that $D B P 2^{P T C}$ is still initially recognised as an NMD target in the mutant strains. Thereby, it may be committed to a defective NMD pathway. In that case it would be possible that a defective NMD pathway causes a slower degradation than the functional regular degradation pathway 
(depending on the normal half-life of the mRNA). As we see a stabilisation by the PTC not only in $g b p 2 \Delta h r b 1 \Delta$ but also, and more pronounced, in the absence of Upf1, other factors would need to be able to initiate NMD without Upf1. It was hypothesised that the binding of Upf1 to the terminating ribosome initiates NMD dependent on other factors, such as Upf2 and Upf3 (Kurosaki et al., 2014). It is possible that even before loading of Upf1, the mRNP is committed to the NMD pathway. Previous reports with a PGK1 based NMD reporter showed that the PGK1 mRNA was reduced to $28 \%$ by a PTC and this effect was reverted in a upf1 $\triangle$ upf2 $\triangle$ upf3 $\triangle$ strain. Similar to our findings of $C B P 80^{P T C}$ in upf1 $\triangle$, the $P G K 1 \mathrm{mRNA}$ was unaffected by the PTC without the Upf factors (Gao et al., 2005). To test a possible influence of Upf2 and Upf3, it would be interesting to test $D B P 2^{P T C}$ in upf1 $\triangle$ upf2 $\triangle$ upf3 $\triangle$ cells. There are some indications that Upf2-Upf3 might bind to NMD targets without Upf1. Due to their low cellular abundance, it seems unlikely, that Upf2 and Upf3 bind to all translated mRNPs (Dehecq et al., 2018; Maderazo et al., 2000). NMD occurs on polysomes, but the polysomal association of Upf2 is even increased without Upf1 (Atkin et al., 1997). This indicates that Upf2 might bind NMD substrates independent of Upf1 and normally dissociates during the NMD pathway.

Since $D B P 2^{P T C}$ behaves differently from $C B P 80^{P T C}$ or $P G K 1^{P T C}$, it is possible that this is caused by the exon junction downstream of the PTC. However, there is no Exon Junction Complex described in yeast.

As far as the involvement of Gbp2 and Hrb1 is concerned, both intron containing NMD reporters showed approx. the same effect. The destabilisation by the PTC is diminished in gbp2 $\Delta$ hrb1 $1 \Delta$ cells, and when Upf1 is missing, additional loss of Gbp2 and Hrb1 has no further effect.

In our analysis we related the NMD reporters to the respective control reporter. Nuclear and other non-NMD related effects in gbp2 $\Delta$ hrb1 $\Delta$ should affect both reporters similarly. Thus, we assume the analysis represents specific NMD effects. Nevertheless, loss of Gbp2 and Hrb1 was shown to cause an increased leakage of unspliced pre-mRNA into the cytoplasm. Unspliced mRNAs are also NMD targets, because intron sequences typically contain several PTCs. That means, some pre-mRNA molecules of the wild typical reporters may leak into the cytoplasm and are NMD substrates in gbp2 $\Delta$ rb1 $1 \Delta$ cells. This could affect the results of this analysis. However, the gbp2 $\Delta$ hrb1 $\Delta$ mutant doesn't seem to cause missplicing, but rather seem to be involved only in the nuclear retention and degradation of mRNAs (Hackmann et al., 2014). The mutant doesn't seem to decrease the splicing rate, but rather a fraction of the unspliced pre-mRNAs, which is normally degraded in the nucleus, leaks into the cytoplasm and is degraded there instead. If the level of correctly spliced mRNA is not lowered in gbp2 $\Delta$ hrb1 $1 \Delta$ cells, the mRNA level of the wild typical reporters cannot be reduced NMD dependently. Only an NMD dependently lowered level of the wild 
typical reporters would lead to an artificially increased relative level of the PTC containing reporters. Also, the leakage of pre-mRNA is likely in the range of a few percent (Hackmann et al., 2014). This is consistent with the observation that the levels of wild typical reporters in gbp2 $\Delta$ hrb1 $\Delta$ cells are entirely unaffected by the additional loss of Upf1 (see Figure $27 \mathrm{~A}$ and $B$, page 82 ). Thus, the fraction of mRNAs that leak into the cytoplasm and become NMD targets is neglectable and cannot account for the almost 2 -fold relative enrichment of PTC-mRNAs in the gbp2 $\Delta$ hrb1 $\Delta$ strain compared to wild type (see Figure 8, page 64 and Figure 9, page 65). Further, when we compare the effects of functional Upf1 in wild type cells (compared to upf $1 \Delta$ cells) and gbp2 $\Delta$ hrb1 $\Delta$ cells (compared to upf $1 \Delta$ gbp2 $\Delta$ hrb1 $\Delta$ ) on the PTC containing reporters, we can see that the destabilising effect of Upf1 is reduced, on average, by half (see Figure 10,65). This must be independent of pre-mRNA leakage, as the NMD reporters are always NMD substrates whether or not they are successfully spliced. In conclusion, we can see that Gbp2 and/or Hrb1 are involved in the nonsense mediated decay of the $D B P 2^{P T C}$ and the $C B P 80^{P T C}$ reporter. This effect is, as expected, Upf1 dependent.

As the later experiments suggested that both Gbp2 and Hrb1 are involved in NMD but have different roles (see below), it would also be interesting to repeat this assay in the single GBP2 and HRB1 knockout strains to see which has the greater influence on degradation. It would also be interesting to see how much the effects of the single knockouts accumulate in the double knockout. If the roles of Gbp2 and Hrb1 are mostly complimentary to each other, we would expect that the effect of the double deletion is approximately the sum of the effects of the single knockouts. Their roles might, however, also be partially codependent.

\subsection{Gbp2 and Hrb1 are also involved in translation inhibition of NMD targets}

We have to assume that translation inhibition is an important function of NMD. While for both our reporters the mRNA level was affected rather mildly by NMD, the level of translated protein was strongly reduced by the presence of Upf1 (see below). This agrees with the notion that preventing the synthesis of potentially toxic polypeptides is one of the central benefits of quality control.

When we tested the translation of our NMD reporters, we could see that it is increased in gbp2 $\Delta$ hrb1 $\Delta$ and in upf1 $\Delta$ cells (see Figure 11, page 66 and Figure 12, page 67). Similar results were published, showing that NMD targets are translated when Upf1 is missing (Dehecq et al., 2018; Kim et al., 2017; Kuroha et al., 2009; Muhlrad and Parker, 1999b). The $P G K 1^{P T C}$ reporter showed an increased translation in upf1 $\triangle$ but no influence of Gbp2 or Hrb1 (see Figure 15, page 69). As we could detect no visible bands for MYC-Pgk1 in wild 
type or the GBP2 and $H R B 1$ knockout strains, it is possible that gbp2 $A r b 1 \Delta$ cells have an increased translation, which is still below the detection limit in the Western blot analysis. But even then, an influence of Gbp2 and Hrb1 would appear to be neglectable, which is consistent with previous analysis of the $P G K 1^{P T C}$ mRNA level (unpublished data, laboratory of Heike Krebber). At this point, it seems most likely that Gbp2 and Hrb1 are only relevant for NMD on spliced transcripts. But it is possible that they are relevant for a subset of transcripts that is determined differently. Gbp2 and Hrb1 also bind to transcripts from nonintron containing genes, if at a reduced rate - Gbp2 has a 4-fold and Hrb1 a 2-fold enriched binding to transcripts encoded by intron containing genes (Hackmann et al., 2014). They also appear to have some relevance for both spliced and non-spliced transcripts in the cytoplasm (see Figure 28, page 84 and 5.8).

We could see that the absence of Gbp2 and Hrb1 shows no increased translation without Upf1 (see Figure 13, page 68) or without a PTC (see Figure 14, page 69), suggesting that this is an NMD specific effect.

Interestingly, when we looked at the sizes of the translated proteins, it appears that MYC$D B P 2^{P T C}$ is terminated at the PTC, while the PTCs of MYC-CBP80PTC and MYC-PGK1 $1^{\text {PTC }}$ are read through (in NMD mutants). It was previously observed that mutants of UPF1, UPF2 or UPF3 can cause suppression of some nonsense codons (Wang et al., 2001). It was originally assumed that NMD and nonsense suppression relate to two distinct functions of Upf1. Conversely, experiments with a luciferase based read through reporter showed that loss of human UPF1 caused the opposite phenotype - a decreased stop codon readthrough (Ivanov et al., 2008). This is consistent with a recent study in human cells with CFTR mRNA that contained a PTC at codon 508 of 1480 (Jia et al., 2017). The discrepancy with yeast reports of nonsense suppression was assumed to reflect differences between human and yeast Upf1. However, in both human studies the employed reporter resembles our DBP2PTC reporter in regard to the PTC position. Interestingly, with the $D B P 2^{P T C}$ reporter we could not detect nonsense suppression in upf1 $\Delta$ either. This indicates that the presumed difference between the species might in fact be caused by construct specific differences. Our findings suggest that the observed nonsense suppression might be caused by an inability to terminate translation at some PTCs independent of Upf1. Translation termination is described to be controlled by regulating factors (Cosson et al., 2002; Czaplinski et al., 2000; Roque et al., 2015; Urakov et al., 2001, 2017). Further, translation termination and the distinction between a regular stop codon and a PTC are context dependent (Amrani et al., 2004; Decourty et al., 2014; González et al., 2000, 2001b; Muhlrad and Parker, 1999a). Very likely the ribosome is missing the correct protein context for termination at these PTCs. We assume that the context at these 5' proximal PTCs strongly favours NMD and disfavours regular termination so that even loss of Upf1 cannot cause translation termination at these 
sites. It seems plausible that if neither NMD nor regular termination can occur at a PTC, it is read through. Likely, the ribosome stalls briefly until a near/non-cognate tRNA enters the A-site and translation elongation continues. Interestingly, analysis with HIS3 reporters containing PTCs at different positions showed that translation could terminate at all PTCs, which was increased by UPF1 deletion. (Kuroha et al., 2009). The PTC closest to the Start codon in their analysis was at codon 50 (not counting an N-terminal FLAG tag). The PTCs of $C B P 80^{P T C}$ and $P G K 1^{P T C}$, which were used here, are at codon 26 and 33 , respectively (not counting the N-terminal MYC tag).

Apart from the position of the PTC within the ORF, it seem very likely that factors specific for a certain mRNA also influence NMD. Early studies of NMD in yeast have identified several sequence specific elements that can either induce or inhibit NMD (Jacobson and Peltz, 2000; Peltz et al., 1993; Ruiz-Echevarria and Peltz, 1996; Ruiz-Echevarría et al., 1998; Zhang et al., 1995). These elements are not very conserved between different mRNAs, indicating that mRNAs are affected differently by PTCs at certain positions. To investigate this phenomenon further, it would be interesting to use CBP80 and PGK1 NMD reporters with PTCs at different positions and determine at which position regular termination can occur. In such an experimental setup, it would also be interesting to see, if there is a distinct position, at which termination at a PTC can occur (in upf1 $\Delta$ ) or if there are intermediate positions, where translation termination and read through at a PTC occur at certain percentages.

Apart from the site of termination, we also found other differences between the MYC$D B P 2^{P T C}$ and the $M Y C-C B P 80^{P T C}$ reporter. The $M Y C-D B P 2^{P T C}$ reporter showed an increased translation in both gbp2 $\triangle$ and $h r b 1 \Delta$, while $M Y C-C B P 80^{P T C}$ translation was only influenced by Gbp2. This indicates that the mechanism is different on the two reporters. Most likely this is due to the PTC being farther downstream in the DBP2 ORF sequence, but it could also be a consequence of its position upstream of the intron (if the exon-exon junction has any relevance in yeast; for the PTC positions see Figure 8 A, page 64 and Figure $9 \mathrm{~A}$, page 65). It could, however, also be caused by other determinants specific to each transcript. This could be tested in an experimental setup with PTCs at different positions as described above. If the position of the PTC is the main determinant, a DBP2 transcript with a PTC close to the start codon should behave like $C B P 80^{P T C}$ (if the intron position is irrelevant). In the following studies to determine the mechanism how Gbp2 and Hrb1 influence NMD, we concentrated on the $C B P 80^{P T C}$ reporter. It would be interesting to see how the $D B P 2^{P T C}$ reporter differs in the NMD mechanism. However, as discussed previously, we assume that the $C B P 80^{P T C}$ reporter is the more typical NMD substrate in yeast and better reflects the situation of endogenous NMD substrates, as the intron and the PTC are relatively close to the start codon. 
To distinguish between effects on translation and on the mRNA level itself, we related the protein levels to the mRNA levels to estimate the translation rate. The translation rates are, however, artificially lowered in the NMD mutants in such an analysis. The translation rates of $M Y C-C B P 80^{P T C}$ are increased $\sim 3.5$-fold in gbp2 $\triangle$ cells and $\sim 10$-fold in upf $1 \Delta$ cells (see Figure $11 \mathrm{C}$, page 66 ), while the level of translated protein (without relating to the mRNA level) is increased $\sim 6$-fold and $\sim 26$-fold, respectively (see Figure $11 \mathrm{~B}$, quantification not shown). The translationally inhibited mRNAs are rapidly degraded and removed from the pool of RNAs in wild type cells. Thereby the calculated translation rate is higher in wild type cells. To analyse translational effects without influence of increased PTC dependent mRNA degradation, it would be possible to repeat the translation analysis in knockouts or mutants of the degradation machinery. However, deletion of $X R N 1$, the main NMD degradation factor, impairs overall cell growth and might affect translation rates indirectly. The analysis, as performed here, is sufficient to generally show the influence of Gbp2, Hrb1 and Upf1 on the translation inhibition in NMD.

In our analysis we can only measure the steady state protein levels to calculate the translation rate. The protein level is, however, also dependent on protein degradation. It was reported that also increased proteasomal degradation of polypeptides from NMD targets occurs (Kuroha et al., 2009). Effects on protein degradation might also affect our translation analysis, but these would still be NMD specific effects. To further analyse the NMD dependent proteasomal degradation with our reporter constructs, it would be possible to repeat the analysis with the proteasomal inhibitor MG132 (as performed in Kuroha et al., 2009). If proteasomal decay is also a crucial factor, addition of MG132 should partially reduce the protein level differences between wild type and mutant cells. At least for the $M Y C-C B P 80^{P T C}$ reporter it is, however, unlikely that NMD dependent proteasomal decay has an influence on the results. The translation product is formed when the PTC is read through and termination occurs at the regular stop codon. It seems unlikely that the NMD pathway would induce protein degradation after translation termination at a regular stop codon. To investigate the role of proteasomal decay, it would also be possible to analyse the ubiquitination of the NMD reporter translation products by mass spectrometry in the different strains.

Gbp2 was implicated in translation regulation before. It was found in P-body dependent cytoplasmic foci of translationally inhibited mRNAs after glucose starvation (Buchan et al., 2008). However, it was not tested if the presence of Gbp2 had an impact on the translation inhibition. Generally, Gbp2 and Hrb1 were suggested to be potential translation inhibitors as they are RGG motif containing proteins. This class of proteins was suggested to be a general group that can inhibit translation initiation. For three proteins, Scd6, Sbp1 and Npl3, it was shown that they inhibit translation (depending on their RGG motifs) through 
interaction with elF4G (Rajyaguru et al., 2012; Segal et al., 2006; Windgassen et al., 2004). Gbp2 and Hrb1 co-precipitate with both elF4E and elF4G after RNase treatment (unpublished data, laboratory of Heike Krebber), supporting a potential role in translation regulation. Gbp2 and Hrb1 physically interact with Npl3, Sbp1 and Scd6 (data not shown; and unpublished data, laboratory of Heike Krebber). We tested if one of these three proteins might mediate the translation inhibition effect of Gbp2, but we found no effect on the translation of the MYC-CBP8O ${ }^{P T C}$ reporter in their absence (see Figure 17, page 71). This suggests that for this NMD substrate these translation inhibitors are irrelevant, while Gbp2 is involved instead. Gbp2, as an RGG motif protein, might act as a translational inhibitor itself. It seems plausible that different translation inhibitors act on different subsets of transcripts. However, in our analysis we could not detect any MYC-CBP8O ${ }^{P T C}$ translation product in $n p / 3 \Delta$ cells. We confirmed the NMD reporter on the mRNA level, which was approx. $10 \%$ of the level in wild type cells (data not shown). It is possible that due to the reduced mRNA level, the translation product is below the chemiluminescence detection limit. However, if the translation rate was increased at least 3.5-fold (as in gbp2 $\Delta$ ), the protein level should at least be approx. a third of the wild type level. But even long exposures (data not shown) showed no MYC signal in the $n p / 3 \Delta$ samples, while all other samples showed distinct bands. Hence, the detection limit is very likely not the reason for the missing NMD reporter translation product. As Npl3 appears to be involved in various pathways (Dermody et al., 2008; Kress et al., 2008; Moehle et al., 2012; Windgassen et al., 2004 and unpublished data, laboratory of Heike Krebber), we cannot exclude the possibility that other defects in the absence of Npl3 might overshadow a possible translation inhibition effect. But so far it seems unlikely that Npl3, Sbp1 or Scd6 mediate the Gbp2 dependent translation inhibition effect of $C B P 80^{P T C}$. It is, of course, still possible that other factors mediate this effect. It was recently shown that loss of Ebs 1 or Nmd4 causes increased translation of an NMD reporter and the effect was increased in the double deletion (Dehecq et al., 2018). Similar to our studies, the phenotype was partial compared to upf1 $\Delta$ cells. The protein level was enriched $\sim 2$.5-fold in ebs $1 \Delta n m d 4 \Delta$ and $\sim 26$-fold in upf1 $\Delta$ cells. In our studies, the MYC-Cbp $80^{\text {PTC }}$ protein was enriched $\sim 26$-fold in upf1 $\Delta$ and $\sim 6$-fold in gbp2 $\Delta$ cells (see above). It would be interesting to study if the NMD reporter translation is further increased in ebs1 $\Delta$ md4 4 gbp2 $\Delta$ cells.

The fact that the translation effect of $g b p 2 \Delta$ is partial would suggest that Gbp2 is one of several factors mediating the translation inhibition of Upf1. However, the formation of the Upf1-2/3 complex on the CBP80 ${ }^{P T C}$ reporter is apparently not reduced in the absence of Gbp2 (unpublished data, laboratory of Heike Krebber). Upf1 induces NMD and disassembles the ribosome at the PTC (Serdar et al., 2016). But on this reporter, the ribosome needs to read through the PTC in order to terminate translation. Thus, we have 
to assume that the action of Upf1 reduces the translation rate in $g b p 2 \Delta$ by disassembling ribosomes at the PTC. We found low amounts of translated MYC-Cbp80 ${ }^{\text {PTC }}$ protein also in wild type cells, indicating that the NMD pathway occasionally fails to recognise the PTC, allowing the read through in wild type cells. In yeast, it was shown that NMD, including degradation processes, occurs on polysomes (Atkin et al., 1997; Hu et al., 2010; Zhang et al., 1997), indicating that several ribosomes can enter translation before NMD takes effect. Likely, in gbp2 $\Delta$ the NMD pathway fails to inhibit the translation initiation correctly, leading to more ribosomes that enter translation on this transcript until mRNA degradation begins. But still, each of these ribosomes might only have a certain chance of running through the PTC when Upf1 is present. Thus, it would theoretically be possible that Gbp2 is the main factor mediating the translation inhibition on this reporter, even though the effect on the reporter translation is lower in gbp2 $\Delta$ than in upf1 $\Delta$ cells.

\subsection{The binding of elF4E to $C B P 80^{P T C}$ is not regulated by the Upf1 pathway}

While NMD is associated with the CBC in higher eukaryotes (Ishigaki et al., 2001; Lejeune et al., 2002), it is not clear in yeast. It was shown that loss of the CBC had no effect on NMD (Gao et al., 2005). The CBC is mainly nuclear and shows little polysomal association (Görlich et al., 1996; Windgassen et al., 2004), while elF4E is the main cap binding factor during steady state translation (Fortes et al., 2000; Gingras et al., 1999). The cap binding structure must be changed at some point in the early phase of translation. It is not clear if this change is regulated by NMD or if the cap structure has an effect on a transcript's susceptibility to NMD. When we analysed the binding of elF4E-GFP to the $C B P 80^{P T C}$ reporter, we found that elF4E preferentially binds to the wild typical CBP80 mRNA and this is not altered in gbp2 $\Delta$ or upf1 $\Delta$ cells (see Figure 19, page 73). Thus, the increased translation does not seem to be caused by an increased association of elF4E. It was previously reported that binding of a PGK1 based NMD reporter to both the CBC and elF4E was reduced to approx. $20 \%$ relative to wild typical PGK1 mRNA. This reduction was reverted in upf1 $\Delta$ upf2 $\Delta$ upf3 $\Delta$ (Gao et al., 2005). However, in their analysis they did not relate the eluted RNA levels to the lysate RNA levels. In fact, in their analysis the PGK1 ${ }^{\text {PTC }}$ to PGK1 ratio of the eluates was identical to the ratio in the lysates in all samples. This means in their studies both the CBC and elF4E showed actually no preferential binding to PGK1 with or without the PTC. In initial RIPs of elF4E-GFP, without UV-crosslinking, we received similar results (data not shown). However, we have to assume that this is due to elF4E binding to the proteins of the mRNP rather than the RNA itself. With UV-crosslinking and stringent wash conditions, elF4E showed a reduced binding of the $C B P 80^{P T C} \mathrm{NMD}$ reporter compared to wild typical CBP80 mRNA. This suggests that NMD on this transcript 
is rather associated with the CBC.

Surprisingly, the UPF1 knockout did not raise the NMD reporter binding to the level of the CBP80 mRNA. That means other factors must still distinguish the NMD reporter from the wild typical mRNA. This might be a consequence of the inefficient translation of the NMD reporter even in upf1 $\Delta$ cells (see Figure 18, page 73). The inefficient translation might be caused by the read through of the PTC, assuming that this is less efficient than regular translation elongation. A low translation efficiency might trigger a reduced binding of elF4E as part of a regulatory mechanism. It was published that inefficient translation is recognised in the cell, however this was described to be Upf1 mediated (Celik et al., 2017), which cannot be the case here. It would be interesting to test the upf1 $\Delta$ upf2 $\Delta$ upf3 $\Delta$ triple deletion, to see if Upf2-Upf3 might have a role in such a regulation. Translation initiation in association with the CBC is described to be less efficient than with elF4E (Fortes et al., 2000). That means, independent of whether or not the elF4E binding is regulated by the translation efficiency, the reduced switch to elF4E would (further) reduce the translation efficiency due to the lower rate of initiation. It is possible that the translation efficiency is in fact not drastically lowered by the read through of the PTC, but rather a consequence of reduced elF4E binding (if not a combination of both). If the read through of the PTC is indeed relevant, the $D B P 2^{P T C}$ reporter translation and eIF4E binding should be like wild typical $D B P 2$ mRNA in upf1 $\triangle$. Therefore, it would be interesting to repeat these analyses with the $D B P 2^{P T C}$ reporter.

Further, it is possible that other NMD factors reduce the translation efficiency. As discussed for $D B P 2^{P T C}$ in 5.2, it is possible that other factors initiate NMD before Upf1 binds. Thus, the transcript might already be determined to be in the NMD pathway, only that the NMD pathway doesn't work effectively as the main factor, Upf1, is missing. It would be interesting to test if in the absence of Upf1 the additional knockout of UPF2 and UPF3 might have an effect on the translation and elF4E binding of $C B P 80^{P T C}$.

In 2 out of 4 experiments the eluted $C B P 80^{P T C}$ reporter showed the same precipitation in the elF4E pulldowns compared to empty beads. It is possible that $C B P 80^{P T C}$ has in fact no direct binding to elF4E. In our experiments $1 \mathrm{M} \mathrm{NaCl}$ did not wash off $C B P 80^{P T C}$ or $C B P 80$ from empty GFP selector beads and increasing the number of washing steps showed no difference. Likely, the unspecific binding to the beads is not a polar or ionic interaction. In our procedure the high salt washing steps were followed by low salt washing steps, prior to proteinase $\mathrm{K}$ digestions. It is possible that in the low salt washing steps RNAs might associate via polar interactions with precipitated proteins in the elF4E pulldowns (given the locally increased concentration on the beads, but dependent on the binding affinity) and finally show a higher co-precipitation with elF4E. To understand the elF4E binding to the NMD reporter beyond doubt, it is necessary to optimize the stringency of the RIP. The 
interaction of the GFP tag to the GFP selector beads is stable under very stringent conditions and crosslinked interactions of elF4E to the RNA should not be subject to washing, due to the covalent bond of the crosslink. Stringent washing conditions that better remove the RNA unspecifically bound to the beads should give better insight into the binding of eIF4E to the NMD reporter.

If there is eIF4E binding to the NMD reporter in the cell (if at a reduced rate), it would be interesting to see if elF4E might already bind to the NMD reporter and other mRNAs in the nucleus. This possibility was suggested before as the deletion of the $\mathrm{CBC}$ is not lethal and showed no effect on a PGK1 based NMD reporter (Gao et al., 2005; Qiu et al., 2012). It was assumed, that without the $\mathrm{CBC}$, elF4E might bind instead in the nucleus. Further, if the increased translation of the NMD reporter in gbp2 $\Delta$ and upf1 $\Delta$ cells is CBC associated, precipitation of the CBC in these mutants should show increased binding to the NMD reporter. This analysis might have to be performed in the absence of Xrn1. The CBC binds mRNAs already in the nucleus, and PTC containing reporter mRNAs are likely rapidly degraded after the $\mathrm{CBC}$ is removed. Deletion of $X R N 1$ or mutants of the decapping enzyme should stabilise the reporter transcripts in the cytoplasm after dissociation of the CBC. Further, we might get better insight into the CBC binding in the cytoplasm by removal of nuclear reporter mRNA. This can either be achieved by cytoplasmic fractionation or by a stop of the GAL1 promotor induced transcription (by adding glucose) and incubation until most of the reporter mRNA has left the nucleus. However, even in the absence of Xrn1, exosomal RNA degradation in the cytoplasm would still remove reporter RNA from the cell over time. Hence, optimising the experimental procedure might be critical if the reporter transcription is stopped by addition of glucose.

Independent of elF4E or the $\mathrm{CBC}$, an increased translation initiation on the $C B P 80^{P T C}$ reporter in gbp2 $\Delta$ and upf1 $\Delta$ cells should be reflected by an increased association of the NMD reporter with the translation initiation machinery. A RIP with elF4G could clarify this, as it is part of the translation initiation machinery regardless of the cap binding proteins (Fortes et al., 2000).

\subsection{Gbp2 and Hrb1 are directly involved in the NMD pathway}

To test whether Gbp2 and Hrb1 are directly involved in the cytoplasm or if they act in the nucleus and cause secondary cytoplasmic effects, we disturbed the nuclear re-import of Gbp2-GFP and Hrb1-GFP by NMD. We induced a high expression of $M Y C-C B P 80^{P T C}$ or the $M Y C-D B P 2^{P T C}$ reporter in $x r n 1 \triangle$ cells and could see that the re-import of Gbp2 and Hrb1 into the nucleus is impaired (see Figure 20, page 74 and Figure 21, page 75 ). This effect is Upf1 dependent, indicating that it is indeed the ongoing NMD that disturbs the Gbp2 and 
Hrb1 localisation. However, ongoing NMD alone does not seem to cause this phenotype as the reporters did not induce mis-localisation if Xrn1 was present (data not shown). Likely, the defective NMD reporter degradation in the absence of Xrn1 causes the NMD pathway to be overloaded. It is possible, that Gbp2 and Hrb1 remain in NMD associated complexes in the cytoplasm if the NMD pathway doesn't function properly (or is at least slower than under normal conditions). In our experiments Gbp2-GFP and Hrb1-GFP did not accumulate in P-bodies, which is thought to be the location of degradation for NMD targets (Durand et al., 2007; Sheth and Parker, 2006). Possibly Gbp2 and Hrb1 dissociate from NMD substrates before or while they are moved to P-bodies, implicating them rather in early steps of NMD. However, we don't know if in our analysis the NMD reporters are correctly transported to $\mathrm{P}$-bodies or if this step of the degradation pathway is defective, when NMD is potentially overloaded. Fluorescence in situ hybridisation experiments with fluorescent probes against the NMD reporters could test if the reporter RNA accumulates in P-bodies. For this, GFP tagged P-body marker proteins, such as Dcp1-GFP, could be used (Sheth and Parker, 2003).

It is also known that the Gbp2 and Hrb1 re-import into the nucleus appears to be regulated by phosphorylation of the SR domain (Häcker and Krebber, 2004; Windgassen and Krebber, 2003). Thus, it is also possible that Gbp2 and Hrb1 do not get phosphorylated readily, if they enter a disturbed NMD pathway. It seems plausible that the phosphorylation of Gbp2 and Hrb1 is regulated in a manner that it occurs after they have fulfilled their function in the cytoplasm. Such a regulation might be flawed under these circumstances. It would be interesting to investigate the phosphorylation state under these conditions by mass spectrometry.

In IP experiments with a Upf1 helicase mutant (Upf1-DE572AA), which causes a retention of the terminating ribosome at the PTC (Serdar et al., 2016) and an increased association of NMD factors (Franks et al., 2010), it was found that Gbp2 but not Hrb1 binding to Upf1 is enriched (unpublished data, laboratory of Heike Krebber). This led us to believe that Hrb1 dissociates from the NMD mRNP earlier than Gbp2. Similarly, fewer cells showed mislocalised Hrb1-GFP than Gbp2-GFP (see Figure 20 B, page 74 and Figure 21 B, page 75). However, we also have reason to assume that Hrb1 has a stronger functional association with the cytoplasmic 5'-3' degradation machinery than Gbp2. Hrb1 generally shows a mislocalisation in all $x r n 1 \Delta$ cells, indicating that it might be functionally related to Xrn1 also independent of NMD (as this is also seen without Upf1 and without induction of the NMD reporters). In the NMD pathway, Hrb1 (and not Gbp2) appears to be relevant for recruiting the decapping enzyme to the NMD reporter (further discussed in 5.7). In Upf1-DE572AA, Xrn1 can likely initiate the 5'-3' degradation normally (Serdar et al., 2016). If Hrb1 dissociates after/when the 5'-3' degradation starts, that could explain why Hrb1 showed no 
enriched binding to Upf1-DE572AA but could be enriched in the cytoplasm when $\mathrm{Xrn1}$ is absent.

It would be interesting to test if the induction of the NMD reporters in the Upf1-DE572AA mutant would cause a cytoplasmic mis-localisation of Gbp2 similar to xrn1 $1 \Delta$ cells. Split GFP experiments suggest that Gbp2 is indeed associated with Upf1-DE572AA in the cytoplasm (unpublished data, laboratory of Heike Krebber). We would expect, however, that the Hrb1GFP localisation is unaffected in this setup. This analysis might confirm if the function of Hrb1 is coupled to the Xrn1 degradation (or decapping). Further, it would be possible to perform IPs with Upf1-DE572AA in $x r n 1 \Delta$ cells or decapping mutants to see if the binding of Hrb1 to the Upf1-complex (or Upf1 bound mRNPs) is enriched.

\subsection{Gbp2 and Hrb1 interact with the cytoplasmic degradation machinery}

Analysis of the Upf1 binding to the $M Y C-C B P 80^{P T C}$ reporter indicated that the NMD specific Upf1 loading to the terminating ribosome is unaffected by Gbp2 and Hrb1 (unpublished data, laboratory of Heike Krebber). Hence, we assume that Gbp2 and Hrb1 do not affect the NMD initiation, but rather act after the Upf1-2/3 complex binds to the terminating ribosome. This hypothesis was supported by our finding that Gbp2 and Hrb1 interact with Dcp1-GFP and Ski2-GFP, factors of cytoplasmic 5'-3' and 3'-5' degradation, respectively (see Figure 22, page 77). Gbp2 and Hrb1 co-precipitated with both degradation factors after RNase treatment, although the Gbp2-Dcp1 interaction was only stable with formaldehyde crosslinking. RNase resistant interactions were also found between Xrn1-GFP and both Gbp2 and Hrb1 (unpublished data, laboratory of Heike Krebber). These studies show that Gbp2, Hrb1 and the degradation machinery do not only bind to the same mRNPs but are in the same protein complex or complexes. This fits recent analysis about the complexes of cytoplasmic degradation in association with NMD. It was shown that the degrading factors form RNase resistant protein-protein interactions with Upf1, suggesting that one or more NMD-degradation protein complexes assemble. Interestingly, Gbp2 was also found in their analyses (Dehecq et al., 2018).

While the interactions of Gbp2 and Hrb1 with the degradation factors do not show whether these are relevant for NMD or if these might be different complexes, it is interesting that Gbp2 and Hrb1 physically interact with the cytoplasmic degradation machinery. So far, Gbp2 and Hrb1 were only described to be part of early translation. In vivo studies showed that the Lsm-ring and the decapping enzyme are not bound during translation and only associate with mRNAs after the RNA has been committed to degradation (Ramirez et al., 2002; Schwartz and Parker, 2000; Tharun and Parker, 2001). Thus, these interactions indicate that Gbp2 and Hrb1 are not only bound during regular translation, but also 
associate with the cytoplasmic degradation machinery after an mRNA has entered the degradation process. We tested if the interactions of Gbp2 with the cytoplasmic degradation machinery are increased in the Upf1-DE572AA mutant with expression of the MYCCBP80 ${ }^{P T C}$ reporter. We performed IPs of Gbp2-GFP followed by mass spectrometry analysis to detect the coprecipitated proteins. Initial results, however, appeared to be too inconsistent, at least with label-free quantification, to quantitatively analyse these interactions. It would be possible to repeat this analysis with SILAC (stable isotope labelling by amino acids in cell culture) or possibly by analysing interactions individually by Western blot analysis. However, it is likely more promising to analyse the effects of Gbp2 and Hrb1 by analysing the binding of the degradation machinery to the NMD reporter (as shown here for Dcp1-GFP and Xrn1-GFP, see Figure 24, page 79 and Figure 25, page 80) or by selectively precipitating the NMD reporter for further analysis as described in section 5.10.

\subsection{Hrb1 promotes the recruitment of the decapping enzyme to MYC- CBP80 PTC}

After we found that Gbp2 and Hrb1 physically interact with the cytoplasmic degradation machinery, we tested the hypothesis that Gbp2 and Hrb1 are relevant for recruiting degradation factors to NMD substrates. Interestingly, it appears that only Hrb1, and not Gbp2, promotes the binding of Dcp1 to the NMD reporter (see Figure 24, page 79). The results of the Dcp1-GFP RIP match our initial analysis of the CBP80 mRNA level very well (see Figure 8, page 64). In wild type cells the binding of the wild typical mRNA is approx. $60 \%$ lower than the NMD reporter. This shows that, indeed, the NMD reporter has a stronger association with the degradation machinery. Without Upf1 the binding to Dcp1GFP is equal between $C B P 80^{P T C}$ and $C B P 80$, similar to their steady state RNA levels. Also, both experiments show that in gbp2 $\Delta$ hrb1 $\Delta$ cells the Upf1 mediated effect of the PTC is approx. reduced by half compared to wild type cells.

It appears that Gbp2 and Hrb1 have different roles in the NMD pathway. While Gbp2 appears to be involved in inhibiting translation, Hrb1 seems to promote the recruitment of the decapping enzyme. The fact that gbp2 $\Delta$ cells show no defect in Dcp1 binding likely reflects that on NMD targets Dcp1 binds to the mRNP via protein-protein interactions (Dehecq et al., 2018) before it binds to the cap. Since the translation inhibition appears to be defective without Gbp2, the availability of the 5' end for Dcp1 is likely reduced, due an increased coverage of the 5' end by the translation initiation machinery. The decapping enzyme can only bind to the cap if at least the first 25 nucleotides are accessible (LaGrandeur and Parker, 1998). Thus, we assume that, in gbp2A cells, Dcp1 is recruited to the mRNP normally by the NMD machinery, but its biding to the cap itself might be reduced 
due to the defective translation inhibition.

Interestingly, RIPs of Ski2-GFP suggest that mostly Gbp2 promotes the recruitment of the Ski complex to the NMD reporter (unpublished data, laboratory of Heike Krebber). We propose a model, in which Hrb1 promotes the recruitment of the decapping enzyme, while Gbp2 promotes the recruitment of the Ski complex (see Figure 29 B). Gbp2's role in translation inhibition likely promotes decapping, as translation initiation and decapping are competing pathways (as discussed above). The removal of translation initiation factors at the cap is presumably required for degradation (see Figure $29 \mathrm{C}$ ).

The effect of $g b p 2 \Delta h r b 1 \Delta$ on the Dcp1 and Ski2 recruitment was approx. half of the effect in upf1 $\Delta$ cells. This phenotype appears to be very consistent in all experiments. However, the IP of Dcp1-GFP to test the co-precipitation of Upf1-HA showed that the interaction was only $\sim 25 \%$ reduced and not reduced by half (see Figure 23 , page 78 ). While this might be an effect of binding kinetics, as the proteins might associate and dissociate in vitro, it probably reflects that Gbp2 and Hrb1 are only relevant for NMD on a subset of transcripts. In this analysis we did not use an NMD reporter, hence, the effect likely reflects NMD on endogenous transcripts. The analysis of the $P G K 1$ based NMD reporter suggests that Gbp2 and Hrb1 are dispensable for some NMD targets (see Figure 15, page 69).

It seems, however, that, at least on a subset of transcripts, the role of Gbp2 and Hrb1 in the cytoplasm is similar to their nuclear role in mRNA quality control. In the nucleus they recruit Mtr4 to mRNAs (Hackmann et al., 2014). Mtr4 is part of the TRAMP complex, which is the nuclear cofactor of the exosome. Hence, it is the nuclear counterpart of the Ski complex. Interestingly, Mtr4 and Ski2, which are both RNA helicases, have great structural homology (Halbach et al., 2012; Johnson and Jackson, 2013). This suggests that Gbp2 might act on both proteins similarly. The exact mechanism, however, is also unknown for Mtr4.

In contrast to Dcp1 and Ski2, the RIPs with Xrn1-GFP suggest that the binding of Xrn1 to the NMD reporter is Upf1 dependent but Gbp2 and Hrb1 independent (see Figure 25, page 80). We did expect that Gbp2 and Hrb1 would act rather early in the NMD pathway. However, RNase resistant interaction of Xrn1 with Gbp2 and Hrb1 had initially suggested that they might have a function directly on Xrn1 (unpublished data, laboratory of Heike Krebber).

This is a further functional indication that the NMD associated degradation machinery binds to the mRNP before they perform their enzymatic activity, because the Xrn1 binding appears to be independent of the reduced binding of the decapping enzyme and, generally, the reduced degradation. We assume that Xrn1 binds to the NMD associated mRNP (Upf1 dependently) and acts once the cap is removed. Such a model would be consistent with an RNase resistant interaction of Xrn1 with Upf1 and Nmd4 (Dehecq et al., 2018). That means in $g b p 2 \Delta h r b 1 \Delta$ cells Xrn1 degradation is probably reduced because the decapping is 
defective. It is possible that Gbp2 and Hrb1 promote Xrn1 degradation by other means such as mRNP-remodelling. But given the previous results, decapping seems to be the most likely function in mRNA decay that might be defective in gbp2 $\Delta$ rb1 $\Delta$. Thus, we would assume that $g b p 2 \Delta h r b 1 \Delta$ and both single knockouts lead to decapping defects. We performed initial experiments to test this hypothesis. In order to determine if decapping of the MYC-CBP80 $8 T C$ reporter is defective, we isolated RNA from $x r n 1 \triangle$ cells (and combinations with $g b p 2 \Delta$, hrb1 $\Delta$ or upf1 $\Delta$ ), which expressed the NMD reporter. As Xrn1 can only digest RNA that has an accessible 5' phosphate (Jinek et al., 2011), it cannot degrade capped mRNAs. We performed in vitro RNA digestion with recombinant Xrn1. Determining the level of the NMD reporter before and after the digestion should allow quantification of uncapped RNA (assuming that the digestion is complete). However, in our initial experiments we could not detect an increased decapping of the NMD reporter compared to the wild typical mRNA, or any difference in the absence of Gbp2, Hrb1 or Upf1 (data not shown). Expressing the NMD reporter in the absence of Xrn1 is problematic, because we could see that Gbp2 and Hrb1 mis-localise under these conditions. To overcome this problem, we induced the transcription of the NMD reporter only $15 \mathrm{~min}$, stopped the transcription by adding glucose, and let the cells incubate another $\sim 8 \mathrm{~min}$. It is possible that this time is too short for the NMD reporter to be quantitatively transported into the cytoplasm, enter translation and be decapped. All the $X R N 1$ deletion strains show reduced growth (data not shown) and due to the generally defective degradation, all mRNA associated pathways might function at a reduced rate. In that case, increasing the incubation times would be sufficient for analysing the decapping effect. However, Xrn1 appears to bind to the NMD associated mRNP before degradation and potentially before decapping. It is possible that Xrn1 binding is structurally relevant for decapping on NMD targets. If binding of Xrn1 is indeed required for efficient decapping, it would be possible to repeat this analysis with a catalytically inactive mutant of Xrn1 (Solinger et al., 1999). We have to assume that the quantification of decapping is not possible if wild typical Xrn1 is present in the cells. Presumably, Xrn1 readily degrades any decapped mRNA, especially if it is already bound to the NMD substrate before decapping.

The verification of a decapping defect in $g b p 2 \Delta, h r b 1 \Delta$ and the double deletion is crucial to the understanding of the NMD mechanism. If Gbp2 and Hrb1 indeed affect decapping by different means, we should expect the effects of the single mutants to add up in the double deletion strain. 


\subsection{Gbp2 and Hrb1 promote the stability of normal mRNAs in the cytoplasm}

Beside the effect of Gbp2 and Hrb1 on NMD transcripts, we saw that the loss of the two proteins leads to a reduced level of wild typical mRNAs (see Figure $27 \mathrm{~A}$ and B, page 82). Surprisingly, this seems to be an effect of mRNA stability caused by Xrn1 degradation (see Figure 28, page 84). Both spliced and unspliced mRNAs showed a $10-30 \%$ reduced RNA level, which was Xrn1 dependent in every case. Surprisingly, the HEM15 and ACT1 mRNAs showed an increased mRNA level through loss of Gbp2 and Hrb1 in the absence of Xrn1. This might be a secondary effect on transcriptional level. Relative to the $x r n 1 \Delta$ control strains, these two mRNAs might be destabilised more strongly than the others. As both spliced and unspliced mRNAs are affected, it cannot be a consequence of their nuclear role in splicing quality control. Also, the effect is Upf1 independent (see Figure 27 A and B, page 82). We cannot entirely rule out the possibility that Gbp2 and Hrb1 might have an unknown nuclear function on all mRNAs, which might cause cytoplasmic degradation by Xrn1 after mRNA export. However, Gbp2 and Hrb1 are known to be part of the mRNP during translation on many mRNAs (Windgassen et al., 2004). As the protein structure covering an mRNA is known be crucial for mRNA stability, it seems most likely that Gbp2 and Hrb1 contribute to the mRNP structure and thereby the mRNA stability (as depicted in our model, see Figure 29 D). Gbp2 and Hrb1 preferentially bind mRNAs closer to their 5 ' ends (Baejen et al., 2014; Tuck and Tollervey, 2013). Thus, if the mRNP structure at the 5' end is disturbed by the absence of the two proteins, the mRNA might be more prone to degradation by Xrn1. Xrn1 is mainly cytoplasmic (located at P-bodies) but was implicated in nuclear rRNA processing together with its homologue Rat1 (Geerlings et al., 2000). If the increased degradation of Xrn1 is indeed occurring in the cytoplasm, it would be interesting to test if an mRNA export block with a Mex67 mutant would also revert the phenotype. Further, it would be interesting to see how the mRNA levels are reduced in the single GBP2 and HRB1 knockout strains. Our other results suggest that it is rather Hrb1 that is functionally associated with the Xrn1 degradation pathway. Likewise, the destabilisation of wild typical mRNAs in gbp2 $\Delta$ rb1 $\Delta$ cells might be mostly Hrb1 dependent.

\subsection{Gbp2 and Hrb1 may contribute to the mRNP structure}

If NMD is initiated at the terminating ribosome at the PTC and ends with the degradation of the mRNA at the 5' and 3' ends, the pathway must be able to bridge this distance. Upf1DE572AA, which fails to dissociate the ribosome and is presumably stuck in the NMD complex, seems to be able to induce degradation by Xrn1 (Franks et al., 2010; Serdar et al., 2016). Either Upf1 must be able to mediate the recruitment of the degradation machinery 
while being bound to the ribosome. Or in addition to the Upf1-2/3 complex at the ribosome, a second Upf1 complex might form on a different Upf1 molecule to recruit the degradation machinery. Either way, the information must be passed on from the PTC to the ends of the mRNA. Very likely the three-dimensional structure of the mRNP is essential for this. It is possible that through the correct mRNP remodelling the PTC gets in proximity to the 5' and 3 ' ends (as drawn in Figure 29, page 106). In higher eukaryotes, it was already suggested that Upf1, at the terminating ribosome, contacts the 5' end through mRNP rearrangement (Maquat et al., 2010). The recruitment of Dcp1 and Ski2 to the MYC-CBP80 ${ }^{\text {PTC }}$ reporter is reduced in the absence of Gbp2 and Hrb1. It is possible that Gbp2 and Hrb1 recruit the degradation machinery through direct protein-protein interactions. As the effects of $g b p 2 \Delta$ hrb1 $\Delta$ are only partial, that would mean that the degradation factors can be recruited by interactions with several proteins. If one of these interaction partners is missing, the degradation factor might still bind to the mRNP (at a reduced rate) via a different interaction partner. It would, however, also be possible that Gbp2 and Hrb1 do not recruit the degradation machinery through direct interactions, but rather contribute to the correct mRNP remodelling required for the NMD pathway. Gbp2 and Hrb1 can interact with each other and also with other Gbp2 and Hrb1 molecules (see Figure 26, page 81). Such interactions might contribute to the compaction of the mRNA. A similar function was described for Nab2, a different nuclear guard protein in yeast (Nab2 also has RGG motifs but no SR rich region; Aibara et al., 2017). It was not tested if the Nab2 mediated compaction has an influence on NMD.

Gbp2 and Hrb1 interact with Upf1 and the ribosome, and split GFP analysis with Gbp2 and Upf1 showed that they are in close proximity in the cell during NMD (unpublished data, laboratory of Heike Krebber). Further, Gbp2 and Hrb1 bind mRNAs closer to the 5' end (Baejen et al., 2014; Tuck and Tollervey, 2013) and they interact with the 5' translation initiation machinery (unpublished data, laboratory of Heike Krebber). Thus, it is tempting to speculate that they might help to bridge the distance between the PTC and the 5' end. Gbp2 also interacts with the same domain of Pab1 as Upf1 in vivo (Richardson et al., 2012). Thus, it could also be involved in bridging the gap between the PTC and the 3' end. Pab1 initially inhibits NMD, but it seems likely that, after NMD initiation, the Upf1-complex must contact the 3' end to induce deadenylation and 3'-5' decay. Finally, Gbp2 and Hrb1 might also have some relevance for the mRNA circularisation or the correct conformation of the protein complex that connects 5' and 3' ends. We could see that loss of Gbp2 and Hrb1 leads to increased degradation of wild typical mRNAs by Xrn1 (see 5.8). The main inhibitor of normal 5'-3' decay is likely the interaction of Pab1 with the 5' end (Caponigro and Parker, 1995; Wilusz et al., 2001). As Gbp2 and Hrb1 interact with elF4E, elF4G and Pab1 (unpublished data, laboratory of Heike Krebber), they might be part of the general mRNP structure that 
promotes the correct interaction of Pab1 with the 5' end. Such a role in the correct mRNP conformation at 5' and 3' ends would explain how Gbp2 and Hrb1 have a stabilising effect for wild typical mRNAs but promote the NMD induced destabilisation (depicted in our model, see Figure 29 B and D).

If Gbp2 and Hrb1 contribute to mRNP remodelling, that does not rule out the possibility that they might additionally be part of a binding platform for degradation factors in the NMD pathway. Understanding the mRNP structure and the direct interaction partners of Gbp2 and Hrb1 on NMD targets is crucial to the understanding of their mechanism in NMD (as discussed in 5.10).

\subsection{The mechanism of Gbp2 and Hrb1 in NMD}

We have found out that Gbp2 and Hrb1 continue their quality control function in the cytoplasm. We could see that they are involved in NMD for a subset of transcripts. We have gained insight into how their mechanism might work (as depicted in Figure 29), but much is still unknown. It would be possible to repeat the interaction studies and RIP analysis, which were performed with Dcp1, Ski2 and Xrn1, with other factors of the degradation machinery. Such an approach would, step by step, show which degradation factors have interactions with Gbp2 and/or Hrb1 and, further, if their recruitment depends on the two SR proteins. However, to get a better understanding of the mechanism of Gbp2 and Hrb1, it might prove more helpful to precipitate the NMD reporter directly. Several assays based on peptides interacting with specific RNA sequences are known, such as the $\lambda \mathrm{N}$ peptide and its binding site - boxB (Baron-Benhamou et al., 2004). Such a binding sequence could be used to specifically precipitate an NMD reporter. A different approach might be using immobilised DNA oligonucleotides. DNA sequences that anneal specifically to the NMD reporter might be used for precipitation. While the precipitation of NMD reporters might require extensive optimisation, it could prove very beneficial for the understanding of the NMD pathway. Protein interactions of Gbp2 and Hrb1 on the NMD reporter might be analysed similarly to a tandem affinity purification. A purification of the NMD reporter could be followed by a regular Gbp2-GFP or Hrb1-GFP immunoprecipitation. In such an experimental setup we could analyse interactions specifically on the NMD reporter. Partial crosslink of proteinprotein interactions followed by highly stringent wash conditions could help to find out which interactions are direct. Proteins directly bound to Gbp2 or Hrb1 should have a higher probability to be crosslinked to them than indirectly bound proteins, as for the latter, two or more protein-protein interactions have to be crosslinked. The combination of such an approach with mass spectrometry to identify potentially every protein bound to Gbp2 or Hrb1 might shed light on their function. Direct protein-protein interactions can also be tested 
in vitro with purified proteins, but these lack the NMD context and possibly the correct modifications. Further, purification of an NMD reporter, followed by mass spectrometric analysis of the mRNP in wild type and gbp2 $h r b 1 \Delta$ cells, might show how Gbp2 and Hrb1 influence the mRNP. Mass spectrometric analysis might also show differences in the proteins' modification states. To precipitate an NMD reporter, sequence specifically, the best solution might be using a unique sequence that is cloned within the open reading frame, but not in the vicinity of the PTC. Using a sequence within the 5' or 3' UTR might interfere with the analysis as regulatory elements bind to the untranslated regions of mRNAs (Wilkie et al., 2003; Wilson and Brewer, 1999). The selective precipitation of NMD reporters could also be used to study how other factors are involved in the NMD pathway.

Another aspect of understanding how Gbp2 and Hrb1 function on NMD targets is to understand where on the NMD substrate they bind. It would be possible to express several NMD reporters and perform PAR-CLIP (Photoactivatable-Ribonucleoside-Enhanced Crosslinking and Immunoprecipitation) analysis with Gbp2-GFP and Hrb1-GFP. For such an experiment it might be crucial to enrich those NMD reporter RNAs that are in the process of NMD. This might be achieved by precipitating Upf1, followed by a precipitation of Gbp2 or Hrb1. Another possibility would be cytoplasmic fractionation or the stop of NMD-reporter transcription followed by further incubation until the NMD reporter RNAs have mostly exited the nucleus (as discussed in 5.4).

The physical and functional association of Gbp2 and Hrb1 with the cytoplasmic degradation machinery is a new facet in understanding the mRNA life cycle. It may be a general pattern of nuclear quality control factors that some of them continue their surveillance function in the cytoplasm. As Gbp2 and Hrb1 are apparently promoting the degradation machinery, rather than the detection of premature termination, they might also perform this function for other cytoplasmic surveillance pathways. In higher eukaryotes, SR proteins also affect NMD, but the mechanisms are not understood. The SR-related protein RNPS1 very likely acts in NMD as part of the EJC (Lykke-Andersen et al., 2001). Overexpression of the shuttling SRSF1 or the non-shuttling SRSF2 induces NMD (Zhang and Krainer, 2004). SRSF1 may induce NMD indirectly by promoting translation (Sanford, 2004; Sato et al., 2008), but it may also recruit of UPF1 (Aznarez et al., 2018). The EJC associates with many SR and SR-related proteins (Singh et al., 2012), which may have additional roles in NMD. The role of yeast Gbp2 and Hrb1 is likely different from human SRSF1, as they don't seem to promote translation or the recruitment of Upf1. Their association with the degradation machinery might also serve as an interesting new perspective in the complex network of SR proteins, alternative splicing and NMD in metazoans. 

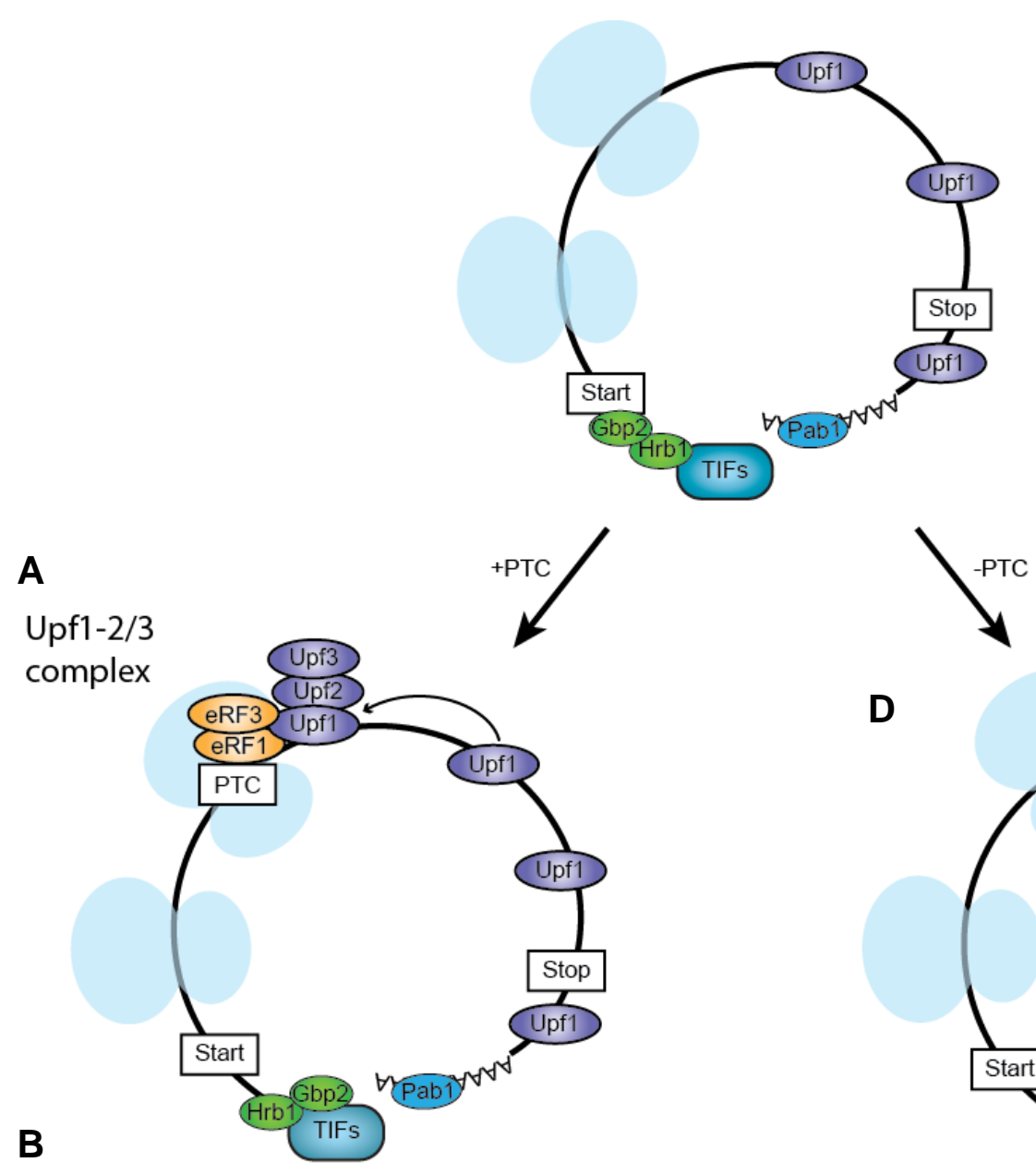

Upf1-decapping

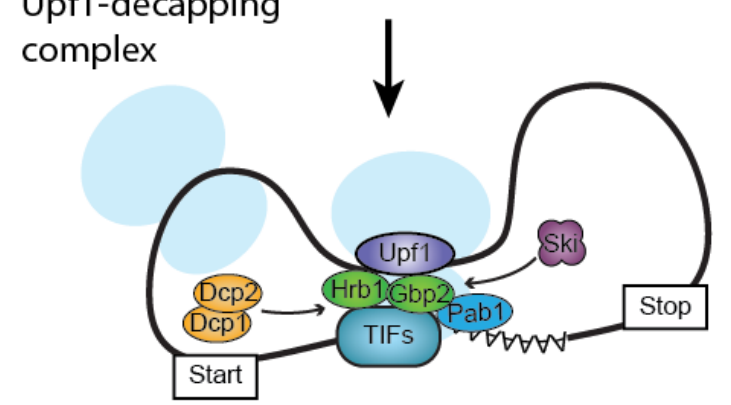

C

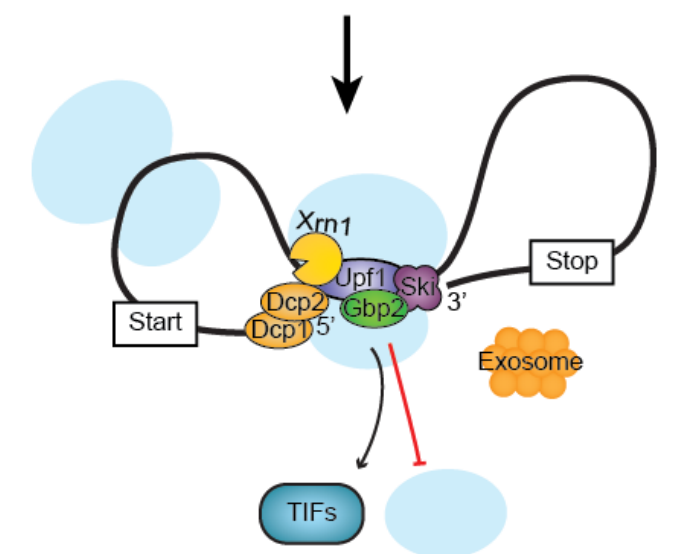

D

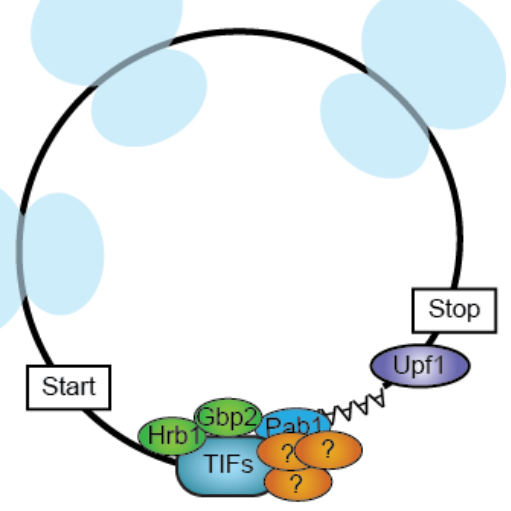

(figure legend on the next page) 


\section{Figure 29: Model of Gbp2 and Hrb1 in NMD}

A

The Upf1-2/3 complex is loaded to the terminating ribosome at the PTC. This initiates the NMD pathway. This step does not depend on Gbp2 and Hrb1, but they might already be part of the complex.

B

Upf1 forms a complex with the degradation machinery. The NMD pathway must bridge the gap between the PTC and the ends of the RNA, likely through mRNP remodelling. Gbp2 and Hrb1 bind close to the 5' ends of RNAs and interact with elF4G, each other and themselves. Gbp2 also interacts with Pab1. They might contribute to an mRNP conformation that brings the Upf1 complex in proximity to the 5' and 3' ends of the mRNA. Further, Hrb1 promotes the recruitment of the decapping enzyme and Gbp2 promotes the recruitment of the Ski complex.

C

Xrn1 is recruited to Upf1 independent of Gbp2 and Hrb1. Likely, Hrb1 dissociates earlier than Gbp2. Gbp2 facilitates translation inhibition. When translation initiation stops and the initiation factors (collectively called TIFs here) vacate the 5' end of the mRNA and the decapping enzyme can remove the cap. Finally, Xrn1 and the exosome can degrade the mRNA. Upf1 mediates the dissociation of the ribosome from the PTC.

D

Without a PTC, the translating ribosome removes Upf1 from the coding region. Translation terminates normally. Gbp2 and Hrb1 stabilise RNAs under these conditions. They might contribute to the correct mRNP conformation - possibly by promoting the correct circularisation or mRNP structure at the 5 ' end. 


\section{References}

Aibara, S., Gordon, J.M.B., Riesterer, A.S., McLaughlin, S.H., and Stewart, M. (2017). Structural basis for the dimerization of Nab2 generated by RNA binding provides insight into its contribution to both poly $(A)$ tail length determination and transcript compaction in Saccharomyces cerevisiae. Nucleic Acids Res. 45, 1529-1538.

Alegria-Schaffer, A. (2014). Western blotting using chemiluminescent substrates. In Methods in Enzymology, (Academic Press Inc.), pp. 251-259.

Alkalaeva, E.Z., Pisarev, A. V, Frolova, L.Y., Kisselev, L.L., and Pestova, T. V (2006). In vitro reconstitution of eukaryotic translation reveals cooperativity between release factors eRF1 and eRF3. Cell 125, 1125-1136.

Allmang, C., Kufel, J., Chanfreau, G., Mitchell, P., Petfalski, E., and Tollervey, D. (1999). Functions of the exosome in rRNA, snoRNA and snRNA synthesis. EMBO J. 18, 53995410.

Altamura, N., Groudinsky, O., Dujardin, G., and Slonimski, P.P. (1992). NAM7 nuclear gene encodes a novel member of a family of helicases with a Zn-ligand motif and is involved in mitochondrial functions in Saccharomyces cerevisiae. J. Mol. Biol. 224, 575-587.

Amrani, N., Ganesan, R., Kervestin, S., Mangus, D.A., Ghosh, S., and Jacobson, A. (2004). A faux 3'-UTR promotes aberrant termination and triggers nonsense-mediated mRNA decay. Nature 432, 112-118.

Amrani, N., Sachs, M.S., and Jacobson, A. (2006). Early nonsense: mRNA decay solves a translational problem. Nat. Rev. Mol. Cell Biol. 7, 415-425.

Amrani, N., Ghosh, S., Mangus, D.A., and Jacobson, A. (2008). Translation factors promote the formation of two states of the closed-loop mRNP. Nature 453, 1276-1280.

Araki, Y., Takahashi, S., Kobayashi, T., Kajiho, H., Hoshino, S., and Katada, T. (2001). Ski7p G protein interacts with the exosome and the Ski complex for 3'-to-5' mRNA decay in yeast. EMBO J. 20, 4684-4693.

Atkin, A.L., Schenkman, L.R., Eastham, M., Dahlseid, J.N., Lelivelt, M.J., and Culbertson, M.R. (1997). Relationship between yeast polyribosomes and Upf proteins required for nonsense mRNA decay. J. Biol. Chem. 272, 22163-22172. 
Aznarez, I., Nomakuchi, T.T., Tetenbaum-Novatt, J., Rahman, M.A., Fregoso, O., Rees, H., and Krainer, A.R. (2018). Mechanism of Nonsense-Mediated mRNA Decay Stimulation by Splicing Factor SRSF1. Cell Rep. 23, 2186-2198.

Badis, G., Saveanu, C., Fromont-Racine, M., and Jacquier, A. (2004). Targeted mRNA Degradation by Deadenylation-Independent Decapping. Mol. Cell 15, 5-15.

Baejen, C., Torkler, P., Gressel, S., Essig, K., Söding, J., and Cramer, P. (2014). Transcriptome Maps of mRNP Biogenesis Factors Define Pre-mRNA Recognition. Mol. Cell $55,745-757$.

Baierlein, C., Hackmann, A., Gross, T., Henker, L., Hinz, F., and Krebber, H. (2013). Monosome formation during translation initiation requires the serine/arginine-rich protein Npl3. Mol. Cell. Biol. 33, 4811-4823.

Baron-Benhamou, J., Gehring, N.H., Kulozik, A.E., and Hentze, M.W. (2004). Using the lambdaN peptide to tether proteins to RNAs. Methods Mol. Biol. 257, 135-154.

Barthelme, D., Dinkelaker, S., Albers, S.-V., Londei, P., Ermler, U., and Tampé, R. (2011). Ribosome recycling depends on a mechanistic link between the FeS cluster domain and a conformational switch of the twin-ATPase ABCE1. Proc. Natl. Acad. Sci. U. S. A. 108, 32283233.

Becker, T., Franckenberg, S., Wickles, S., Shoemaker, C.J., Anger, A.M., Armache, J.-P., Sieber, H., Ungewickell, C., Berninghausen, O., Daberkow, I., et al. (2012). Structural basis of highly conserved ribosome recycling in eukaryotes and archaea. Nature 482, 501-506.

Beißel, C., Neumann, B., Unse, S., Hampe, I., Karki, P., and Krebber, H. (2019). Translation termination depends on the sequential ribosomal entry of eRF1 and eRF3. Nucleic Acids Res. 47, 4798-4813.

Beznosková, P., Cuchalová, L., Wagner, S., Shoemaker, C.J., Gunišová, S., von der Haar, T., and Valášek, L.S. (2013). Translation initiation factors elF3 and HCR1 control translation termination and stop codon read-through in yeast cells. PLoS Genet. 9, e1003962.

Blencowe, B.J., Issner, R., Nickerson, J.A., and Sharp, P.A. (1998). A coactivator of premRNA splicing. Genes Dev. 12, 996-1009.

Boeck, R., Tarun, S., Rieger, M., Deardorff, J.A., Müller-Auer, S., and Sachs, A.B. (1996). The yeast Pan2 protein is required for poly $(A)$-binding protein-stimulated poly $(A)$-nuclease activity. J. Biol. Chem. 271, 432-438. 
Bolger, T.A., Folkmann, A.W., Tran, E.J., and Wente, S.R. (2008). The mRNA export factor Gle1 and inositol hexakisphosphate regulate distinct stages of translation. Cell 134, 624633.

Bonnerot, C., Boeck, R., and Lapeyre, B. (2000). The Two Proteins Pat1p (Mrt1p) and Spb8p Interact In Vivo, Are Required for mRNA Decay, and Are Functionally Linked to Pab1p. Mol. Cell. Biol. 20, 5939-5946.

Bouveret, E., Rigaut, G., Shevchenko, A., Wilm, M., and Séraphin, B. (2000). A Sm-like protein complex that participates in mRNA degradation. EMBO J. 19, 1661-1671.

Brengues, M., Teixeira, D., and Parker, R. (2005). Movement of Eukaryotic mRNAs Between Polysomes and Cytoplasmic Processing Bodies. Science (80-. ). 310, 486-489.

Brogna, S., and Wen, J. (2009). Nonsense-mediated mRNA decay (NMD) mechanisms. Nat. Struct. Mol. Biol. 16, 107-113.

Brown, C.E., and Sachs, A.B. (1998). Poly(A) tail length control in Saccharomyces cerevisiae occurs by message-specific deadenylation. Mol. Cell. Biol. 18, 6548-6559.

Brown, C.E., Tarun, S.Z., Boeck, R., and Sachs, A.B. (1996). PAN3 encodes a subunit of the Pab1p-dependent poly $(A)$ nuclease in Saccharomyces cerevisiae. Mol. Cell. Biol. 16, 5744-5753.

Brown, J.T., Bai, X., and Johnson, A.W. (2000). The yeast antiviral proteins Ski2p, Ski3p, and Ski8p exist as a complex in vivo. RNA 6, 449-457.

Brune, C., Munchel, S.E., Fischer, N., Podtelejnikov, A. V, and Weis, K. (2005). Yeast poly(A)-binding protein Pab1 shuttles between the nucleus and the cytoplasm and functions in mRNA export. RNA $11,517-531$.

Buchan, J.R., Muhlrad, D., and Parker, R. (2008). P bodies promote stress granule assembly in Saccharomyces cerevisiae. J. Cell Biol. 183, 441-455.

Bucheli, M.E., and Buratowski, S. (2005). Npl3 is an antagonist of mRNA 3' end formation by RNA polymerase II. EMBO J. 24, 2150-2160.

Callahan, K.P., and Butler, J.S. (2010). TRAMP complex enhances RNA degradation by the nuclear exosome component Rrp6. J. Biol. Chem. 285, 3540-3547.

Cao, D., and Parker, R. (2003). Computational modeling and experimental analysis of nonsense-mediated decay in yeast. Cell 113, 533-545. 
Caponigro, G., and Parker, R. (1995). Multiple functions for the poly(A)-binding protein in mRNA decapping and deadenylation in yeast. Genes Dev. 9, 2421-2432.

Celik, A., Baker, R., He, F., and Jacobson, A. (2017). High-resolution profiling of NMD targets in yeast reveals translational fidelity as a basis for substrate selection. RNA 23, 735748.

Chakrabarti, S., Jayachandran, U., Bonneau, F., Fiorini, F., Basquin, C., Domcke, S., Le Hir, H., and Conti, E. (2011). Molecular mechanisms for the RNA-dependent ATPase activity of Upf1 and its regulation by Upf2. Mol. Cell 41, 693-703.

Chávez, S., Beilharz, T., Rondón, A.G., Erdjument-Bromage, H., Tempst, P., Svejstrup, J.Q., Lithgow, T., and Aguilera, A. (2000). A protein complex containing Tho2, Hpr1, Mft1 and a novel protein, Thp2, connects transcription elongation with mitotic recombination in Saccharomyces cerevisiae. EMBO J. 19, 5824-5834.

Christie, K.R., Weng, S., Balakrishnan, R., Costanzo, M.C., Dolinski, K., Dwight, S.S., Engel, S.R., Feierbach, B., Fisk, D.G., Hirschman, J.E., et al. (2004). Saccharomyces Genome Database (SGD) provides tools to identify and analyze sequences from Saccharomyces cerevisiae and related sequences from other organisms. Nucleic Acids Res. 32, D311-4.

Clarkson, B.K., Gilbert, W. V, and Doudna, J.A. (2010). Functional overlap between elF4G isoforms in Saccharomyces cerevisiae. PLoS One 5, e9114.

Coller, J., and Parker, R. (2005). General Translational Repression by Activators of mRNA Decapping. Cell 122, 875-886.

Coller, J.M., Tucker, M., Sheth, U., Valencia-Sanchez, M.A., and Parker, R. (2001). The DEAD box helicase, Dhh1p, functions in mRNA decapping and interacts with both the decapping and deadenylase complexes. RNA 7, 1717-1727.

Cosson, B., Couturier, A., Chabelskaya, S., Kiktev, D., Inge-Vechtomov, S., Philippe, M., and Zhouravleva, G. (2002). Poly(A)-Binding Protein Acts in Translation Termination via Eukaryotic Release Factor 3 Interaction and Does Not Influence [PSI+] Propagation. Mol. Cell. Biol. 22, 3301-3315.

Cui, Y., Hagan, K.W., Zhang, S., and Peltz, S.W. (1995). Identification and characterization of genes that are required for the accelerated degradation of mRNAs containing a premature translational termination codon. Genes Dev. 9, 423-436. 
Czaplinski, K., Weng, Y., Hagan, K.W., and Peltz, S.W. (1995). Purification and characterization of the Upf1 protein: a factor involved in translation and mRNA degradation. RNA 1, 610-623.

Czaplinski, K., Ruiz-Echevarria, M.J., Paushkin, S. V, Han, X., Weng, Y., Perlick, H.A., Dietz, H.C., Ter-Avanesyan, M.D., and Peltz, S.W. (1998). The surveillance complex interacts with the translation release factors to enhance termination and degrade aberrant mRNAs. Genes Dev. 12, 1665-1677.

Czaplinski, K., Majlesi, N., Banerjee, T., and Peltz, S.W. (2000). Mtt1 is a Upf1-like helicase that interacts with the translation termination factors and whose overexpression can modulate termination efficiency. RNA 6, 730-743.

Dahlseid, J.N., Puziss, J., Shirley, R.L., Atkin, A.L., Hieter, P., and Culbertson, M.R. (1998). Accumulation of mRNA coding for the ctf13p kinetochore subunit of Saccharomyces cerevisiae depends on the same factors that promote rapid decay of nonsense mRNAs. Genetics 150, 1019-1035.

Das, B., Guo, Z., Russo, P., Chartrand, P., and Sherman, F. (2000). The Role of Nuclear Cap Binding Protein Cbc1p of Yeast in mRNA Termination and Degradation. Mol. Cell. Biol. 20, 2827-2838.

Decker, C.J., and Parker, R. (1993). A turnover pathway for both stable and unstable mRNAs in yeast: Evidence for a requirement for deadenylation. Genes Dev. 7, 1632-1643.

Decourty, L., Doyen, A., Malabat, C., Frachon, E., Rispal, D., Séraphin, B., Feuerbach, F., Jacquier, A., and Saveanu, C. (2014). Long open reading frame transcripts escape nonsense-mediated mRNA decay in yeast. Cell Rep. 6, 593-598.

Dehecq, M., Decourty, L., Namane, A., Proux, C., Kanaan, J., Le Hir, H., Jacquier, A., and Saveanu, C. (2018). Nonsense-mediated mRNA decay involves two distinct Upf1-bound complexes. EMBO J. 37, e99278.

Dehlin, E., Wormington, M., Körner, C.G., and Wahle, E. (2000). Cap-dependent deadenylation of mRNA. EMBO J. 19, 1079-1086.

Dermody, J.L., Dreyfuss, J.M., Villén, J., Ogundipe, B., Gygi, S.P., Park, P.J., Ponticelli, A.S., Moore, C.L., Buratowski, S., and Bucheli, M.E. (2008). Unphosphorylated SR-like protein Npl3 stimulates RNA polymerase II elongation. PLoS One 3, e3273. 
Dever, T.E., Kinzy, T.G., and Pavitt, G.D. (2016). Mechanism and Regulation of Protein Synthesis in Saccharomyces cerevisiae. Genetics 203, 65-107.

Dower, W.J., Miller, J.F., Ragsdale, C.W., Group, M.B., Laboratories, B., and Medical, S. (1988). High efficiency transformation of E.coli by high voltage electroporation. 16, 61276146.

Dunn, E.F., Hammell, C.M., Hodge, C.A., and Cole, C.N. (2005). Yeast poly(A)-binding protein, Pab1, and PAN, a poly $(\mathrm{A})$ nuclease complex recruited by Pab1, connect mRNA biogenesis to export. Genes Dev. 19, 90-103.

Durand, S., Cougot, N., Mahuteau-Betzer, F., Nguyen, C.-H., Grierson, D.S., Bertrand, E., Tazi, J., and Lejeune, F. (2007). Inhibition of nonsense-mediated mRNA decay (NMD) by a new chemical molecule reveals the dynamic of NMD factors in P-bodies. J. Cell Biol. 178, 1145-1160.

Eberle, A.B., Stalder, L., Mathys, H., Orozco, R.Z., and Mühlemann, O. (2008). Posttranscriptional gene regulation by spatial rearrangement of the 3 ' untranslated region. PLoS Biol. 6, e92.

Eberle, A.B., Lykke-Andersen, S., Mühlemann, O., and Jensen, T.H. (2009). SMG6 promotes endonucleolytic cleavage of nonsense mRNA in human cells. Nat. Struct. Mol. Biol. 16, 49-55.

Erce, M.A., Abeygunawardena, D., Low, J.K.K., Hart-Smith, G., and Wilkins, M.R. (2013). Interactions affected by arginine methylation in the yeast protein-protein interaction network. Mol. Cell. Proteomics 12, 3184-3198.

Estrella, L.A., Wilkinson, M.F., and González, C.I. (2009). The Shuttling Protein Npl3 Promotes Translation Termination Accuracy in Saccharomyces cerevisiae. J. Mol. Biol. 394, 410-422.

Eulalio, A., Behm-Ansmant, I., and Izaurralde, E. (2007). P bodies: at the crossroads of post-transcriptional pathways. Nat. Rev. Mol. Cell Biol. 8, 9-22.

Ford, A.S., Guan, Q., Neeno-Eckwall, E., and Culbertson, M.R. (2006). Ebs1p, a negative regulator of gene expression controlled by the Upf proteins in the yeast Saccharomyces cerevisiae. Eukaryot. Cell 5, 301-312. 
Fortes, P., Inada, T., Preiss, T., Hentze, M.W., Mattaj, I.W., and Sachs, A.B. (2000). The yeast nuclear cap binding complex can interact with translation factor elF4G and mediate translation initiation. Mol. Cell 6, 191-196.

Franks, T.M., Singh, G., and Lykke-Andersen, J. (2010). Upf1 ATPase-dependent mRNP disassembly is required for completion of nonsense- mediated mRNA decay. Cell 143, 938950.

Frolova, L., Le Goff, X., Rasmussen, H.H., Cheperegin, S., Drugeon, G., Kress, M., Arman, I., Haenni, A.-L., Celis, J.E., Phllippe, M., et al. (1994). A highly conserved eukaryotic protein family possessing properties of polypeptide chain release factor. Nature 372, 701-703.

Fromm, S.A., Truffault, V., Kamenz, J., Braun, J.E., Hoffmann, N.A., Izaurralde, E., and Sprangers, R. (2012). The structural basis of Edc3- and Scd6-mediated activation of the Dcp1:Dcp2 mRNA decapping complex. EMBO J. 31, 279-290.

Fukumura, K., Wakabayashi, S., Kataoka, N., Sakamoto, H., Suzuki, Y., Nakai, K., Mayeda, A., and Inoue, K. (2016). The Exon Junction Complex Controls the Efficient and Faithful Splicing of a Subset of Transcripts Involved in Mitotic Cell-Cycle Progression. Int. J. Mol. Sci. 17, 1153.

Gaba, A., Jacobson, A., and Sachs, M.S. (2005). Ribosome occupancy of the yeast CPA1 upstream open reading frame termination codon modulates nonsense-mediated mRNA decay. Mol. Cell 20, 449-460.

Galy, V., Gadal, O., Fromont-Racine, M., Romano, A., Jacquier, A., and Nehrbass, U. (2004). Nuclear Retention of Unspliced mRNAs in Yeast Is Mediated by Perinuclear Mlp1. Cell 116, 63-73.

Gao, Q., Das, B., Sherman, F., and Maquat, L.E. (2005). Cap-binding protein 1-mediated and eukaryotic translation initiation factor $4 \mathrm{E}$-mediated pioneer rounds of translation in yeast. Proc. Natl. Acad. Sci. 102, 4258-4263.

Garfin, D.E. (2009). Chapter 29 One-Dimensional Gel Electrophoresis. In Methods in Enzymology, (Academic Press Inc.), pp. 497-513.

Garneau, N.L., Wilusz, J., and Wilusz, C.J. (2007). The highways and byways of mRNA decay. Nat. Rev. Mol. Cell Biol. 8, 113-126. 
Geerlings, T.H., Vos, J.C., and Raué, H.A. (2000). The final step in the formation of $25 \mathrm{~S}$ rRNA in Saccharomyces cerevisiae is performed by 5'--\&gt;3' exonucleases. RNA 6, 16981703.

Ghosh, A., and Lima, C.D. (2010). Enzymology of RNA cap synthesis. Wiley Interdiscip. Rev. RNA 1, 152-172.

Ghosh, I., Hamilton, A.D., and Regan, L. (2000). Antiparallel leucine zipper-directed protein reassembly: Application to the green fluorescent protein [12]. J. Am. Chem. Soc. 122, 56585659.

Gibson, D.G., Young, L., Chuang, R.-Y., Venter, J.C., Hutchison, C.A., and Smith, H.O. (2009). Enzymatic assembly of DNA molecules up to several hundred kilobases. Nat. Methods 6, 343-345.

Gietz, D., St Jean, A., Woods, R.A., and Schiestl, R.H. (1992). Improved method for high efficiency transformation of intact yeast cells. Nucleic Acids Res. 20, 1425.

Gilbert, W., and Guthrie, C. (2004). The Glc7p Nuclear Phosphatase Promotes mRNA Export by Facilitating Association of Mex67p with mRNA. Mol. Cell 13, 201-212.

Gingras, A.C., Raught, B., and Sonenberg, N. (1999). elF4 initiation factors: effectors of mRNA recruitment to ribosomes and regulators of translation. Annu. Rev. Biochem. 68, 913-963.

González, C.I., Ruiz-Echevarría, M.J., Vasudevan, S., Henry, M.F., and Peltz, S.W. (2000). The Yeast hnRNP-like Protein Hrp1/Nab4 Marks a Transcript for Nonsense-Mediated mRNA Decay. Mol. Cell 5, 489-499.

González, C.I., Bhattacharya, A., Wang, W., and Peltz, S.W. (2001a). Nonsense-mediated mRNA decay in Saccharomyces cerevisiae. Gene 274, 15-25.

González, C.I., Bhattacharya, A., Wang, W., and Peltz, S.W. (2001b). Nonsense-mediated mRNA decay in Saccharomyces cerevisiae. Gene 274, 15-25.

Görlich, D., Kraft, R., Kostka, S., Vogel, F., Hartmann, E., Laskey, R.A., Mattaj, I.W., and Izaurralde, E. (1996). Importin Provides a Link between Nuclear Protein Import and U snRNA Export. Cell 87, 21-32.

Graham, A.C., Kiss, D.L., and Andrulis, E.D. (2006). Differential distribution of exosome subunits at the nuclear lamina and in cytoplasmic foci. Mol. Biol. Cell 17, 1399-1409. 
Graille, M., and Séraphin, B. (2012). Surveillance pathways rescuing eukaryotic ribosomes lost in translation. Nat. Rev. Mol. Cell Biol. 13, 727-735.

Graveley, B.R., and Hertel, K.J. (2005). SR Proteins. In Encyclopedia of Life Sciences, (Chichester: John Wiley \& Sons, Ltd), p.

Green, D.M., Johnson, C.P., Hagan, H., and Corbett, A.H. (2003). The C-terminal domain of myosin-like protein 1 (Mlp1p) is a docking site for heterogeneous nuclear ribonucleoproteins that are required for mRNA export. Proc. Natl. Acad. Sci. U. S. A. 100, $1010-1015$.

Gross, T., Siepmann, A., Sturm, D., Windgassen, M., Scarcelli, J.J., Seedorf, M., Cole, C.N., and Krebber, H. (2007). The DEAD-box RNA helicase Dbp5 functions in translation termination. Science 315, 646-649.

Gudikote, J.P., Imam, J.S., Garcia, R.F., and Wilkinson, M.F. (2005). RNA splicing promotes translation and RNA surveillance. Nat. Struct. Mol. Biol. 12, 801-809.

Häcker, S., and Krebber, H. (2004). Differential export requirements for shuttling serine/arginine-type mRNA-binding proteins. J. Biol. Chem. 279, 5049-5052.

Hackmann, A., Wu, H., Schneider, U.-M., Meyer, K., Jung, K., and Krebber, H. (2014). Quality control of spliced mRNAs requires the shuttling SR proteins Gbp2 and Hrb1. Nat. Commun. 5, 3123.

Hagan, K.W., Ruiz-Echevarria, M.J., Quan, Y., and Peltz, S.W. (1995). Characterization of cis-acting sequences and decay intermediates involved in nonsense-mediated mRNA turnover. Mol. Cell. Biol. 15, 809-823.

Halbach, F., Rode, M., and Conti, E. (2012). The crystal structure of S. cerevisiae Ski2, a DExH helicase associated with the cytoplasmic functions of the exosome. RNA 18, 124134.

He, F., and Jacobson, A. (1995). Identification of a novel component of the nonsensemediated mRNA decay pathway by use of an interacting protein screen. Genes Dev. 9, 437-454.

He, F., Peltz, S.W., Donahue, J.L., Rosbash, M., and Jacobson, A. (1993). Stabilization and ribosome association of unspliced pre-mRNAs in a yeast upf1- mutant. Proc. Natl. Acad. Sci. U. S. A. 90, 7034-7038. 
He, F., Brown, A., and Jacobson, A. (1997). Upf1p, Nmd2p, and Upf3p are interacting components of the yeast nonsense-mediated mRNA decay pathway. Mol. Cell. Biol. 17, $1580-1594$.

He, F., Li, X., Spatrick, P., Casillo, R., Dong, S., and Jacobson, A. (2003). Genome-Wide Analysis of mRNAs Regulated by the Nonsense-Mediated and 5' to 3' mRNA Decay Pathways in Yeast. Mol. Cell 12, 1439-1452.

Hector, R.E. (2002). Dual requirement for yeast hnRNP Nab2p in mRNA poly(A) tail length control and nuclear export. EMBO J. 21, 1800-1810.

Hilleren, P., and Parker, R. (1999). mRNA surveillance in eukaryotes: kinetic proofreading of proper translation termination as assessed by mRNP domain organization? RNA 5, 711719.

Le Hir, H., Izaurralde, E., Maquat, L.E., and Moore, M.J. (2000). The spliceosome deposits multiple proteins 20-24 nucleotides upstream of mRNA exon-exon junctions. EMBO J. 19, 6860-6869.

Le Hir, H., Gatfield, D., Izaurralde, E., and Moore, M.J. (2001). The exon-exon junction complex provides a binding platform for factors involved in mRNA export and nonsensemediated mRNA decay. EMBO J. 20, 4987-4997.

Hocine, S., Singer, R.H., and Grunwald, D. (2010). RNA Processing and Export. Cold Spring Harb. Perspect. Biol. 2, a000752-a000752.

Houalla, R., Devaux, F., Fatica, A., Kufel, J., Barrass, D., Torchet, C., and Tollervey, D. (2006). Microarray detection of novel nuclear RNA substrates for the exosome. Yeast 23, 439-454.

Houseley, J., LaCava, J., and Tollervey, D. (2006). RNA-quality control by the exosome. Nat. Rev. Mol. Cell Biol. 7, 529-539.

Hsu, C.L., and Stevens, A. (1993). Yeast cells lacking 5'--\&gt;3' exoribonuclease 1 contain mRNA species that are poly $(A)$ deficient and partially lack the 5' cap structure. Mol. Cell. Biol. 13, 4826-4835.

Hu, W., Petzold, C., Coller, J., and Baker, K.E. (2010). Nonsense-mediated mRNA decapping occurs on polyribosomes in Saccharomyces cerevisiae. Nat. Struct. Mol. Biol. 17, 244-247. 
Huang, Y., and Steitz, J.A. (2005). SRprises along a messenger's journey. Mol. Cell 17, 613-615.

Huntzinger, E., Kashima, I., Fauser, M., Saulière, J., and Izaurralde, E. (2008). SMG6 is the catalytic endonuclease that cleaves mRNAs containing nonsense codons in metazoan. RNA 14, 2609-2617.

Hurt, E., Luo, M. -j., Rother, S., Reed, R., and Strasser, K. (2004). Cotranscriptional recruitment of the serine-arginine-rich (SR)-like proteins Gbp2 and Hrb1 to nascent mRNA via the TREX complex. Proc. Natl. Acad. Sci. 101, 1858-1862.

Hurt, J.A., Robertson, A.D., and Burge, C.B. (2013). Global analyses of UPF1 binding and function reveal expanded scope of nonsense-mediated mRNA decay. Genome Res. 23, 1636-1650.

Ibrahimo, S., Holmes, L.E.A., and Ashe, M.P. (2006). Regulation of translation initiation by the yeast elF4E binding proteins is required for the pseudohyphal response. Yeast 23, 1075-1088.

Iglesias, N., Tutucci, E., Gwizdek, C., Vinciguerra, P., Von Dach, E., Corbett, A.H., Dargemont, C., and Stutz, F. (2010). Ubiquitin-mediated mRNP dynamics and surveillance prior to budding yeast mRNA export. Genes Dev. 24, 1927-1938.

Inada, T. (2017). The Ribosome as a Platform for mRNA and Nascent Polypeptide Quality Control. Trends Biochem. Sci. 42, 5-15.

Inoue, H., Nojima, H., and Okayama, H. (1990). High efficiency transformation of Escherichia coli with plasmids. Gene 96, 23-28.

Ishigaki, Y., Li, X., Serin, G., and Maquat, L.E. (2001). Evidence for a pioneer round of mRNA translation: mRNAs subject to nonsense-mediated decay in mammalian cells are bound by CBP80 and CBP20. Cell 106, 607-617.

Isken, O., and Maquat, L.E. (2007). Quality control of eukaryotic mRNA: safeguarding cells from abnormal mRNA function. Genes Dev. 21, 1833-1856.

Ivanov, P. V, Gehring, N.H., Kunz, J.B., Hentze, M.W., and Kulozik, A.E. (2008). Interactions between UPF1, eRFs, PABP and the exon junction complex suggest an integrated model for mammalian NMD pathways. EMBO J. 27, 736-747.

Jacobson, A. (2005). The end justifies the means. Nat. Struct. Mol. Biol. 12, 474-475. 
Jacobson, A., and Peltz, S.W. (2000). Destabilization of Nonsense-containing Transcripts in Saccharomyces cerevisiae. Cold Spring Harb. Monogr. Arch. 39, 827-847.

Jaillon, O., Bouhouche, K., Gout, J.-F., Aury, J.-M., Noel, B., Saudemont, B., Nowacki, M., Serrano, V., Porcel, B.M., Ségurens, B., et al. (2008). Translational control of intron splicing in eukaryotes. Nature 451, 359-362.

Jamar, N.H., Kritsiligkou, P., and Grant, C.M. (2018). Loss of mRNA surveillance pathways results in widespread protein aggregation. Sci. Rep. 8, 1-10.

Jia, H., Wang, X., Liu, F., Guenther, U.-P., Srinivasan, S., Anderson, J.T., and Jankowsky, E. (2011). The RNA helicase Mtr4p modulates polyadenylation in the TRAMP complex. Cell 145, 890-901.

Jia, J., Werkmeister, E., Gonzalez-Hilarion, S., Leroy, C., Gruenert, D.C., Lafont, F., Tulasne, D., and Lejeune, F. (2017). Premature termination codon readthrough in human cells occurs in novel cytoplasmic foci and requires UPF proteins. J. Cell Sci. 130, 30093022.

Jinek, M., Coyle, S.M., and Doudna, J.A. (2011). Coupled 5' nucleotide recognition and processivity in Xrn1-mediated mRNA decay. Mol. Cell 41, 600-608.

Johansson, M.J.O., and Jacobson, A. (2010). Nonsense-mediated mRNA decay maintains translational fidelity by limiting magnesium uptake. Genes Dev. 24, 1491-1495.

Johansson, M.J.O., He, F., Spatrick, P., Li, C., and Jacobson, A. (2007). Association of yeast Upf1p with direct substrates of the NMD pathway. Proc. Natl. Acad. Sci. 104, 2087220877.

Johnson, S.J., and Jackson, R.N. (2013). Ski2-like RNA helicase structures. RNA Biol. 10, 33-43.

Kashima, I., Yamashita, A., Izumi, N., Kataoka, N., Morishita, R., Hoshino, S., Ohno, M., Dreyfuss, G., and Ohno, S. (2006). Binding of a novel SMG-1-Upf1-eRF1-eRF3 complex (SURF) to the exon junction complex triggers Upf1 phosphorylation and nonsensemediated mRNA decay. Genes Dev. 20, 355-367.

Keeling, K.M., Lanier, J., Du, M., Salas-Marco, J., Gao, L., Kaenjak-Angeletti, A., and Bedwell, D.M. (2004). Leaky termination at premature stop codons antagonizes nonsensemediated mRNA decay in S. cerevisiae. RNA 10, 691-703. 
Kervestin, S., and Jacobson, A. (2012). NMD: a multifaceted response to premature translational termination. Nat. Rev. Mol. Cell Biol. 13, 700-712.

Kervestin, S., Li, C., Buckingham, R., and Jacobson, A. (2012). Testing the faux-UTR model for NMD: analysis of Upf1p and Pab1p competition for binding to eRF3/Sup35p. Biochimie 94, 1560-1571.

Khoshnevis, S., Gross, T., Rotte, C., Baierlein, C., Ficner, R., and Krebber, H. (2010). The iron-sulphur protein $R N a s e ~ L$ inhibitor functions in translation termination. EMBO Rep. 11, 214-219.

Kim, W.K., Yun, S., Kwon, Y., You, K.T., Shin, N., Kim, J., and Kim, H. (2017). mRNAs containing NMD-competent premature termination codons are stabilized and translated under UPF1 depletion. Sci. Rep. 7, 15833.

Klauer, A.A., and van Hoof, A. (2012). Degradation of mRNAs that lack a stop codon: a decade of nonstop progress. Wiley Interdiscip. Rev. RNA 3, 649-660.

Klockenbusch, C., and Kast, J. (2010). Optimization of Formaldehyde Cross-Linking for Protein Interaction Analysis of Non-Tagged Integrin $\beta$ 1. J. Biomed. Biotechnol. 2010,113.

Kong, K.-Y.E., Tang, H.-M.V., Pan, K., Huang, Z., Lee, T.-H.J., Hinnebusch, A.G., Jin, D.Y., and Wong, C.-M. (2014). Cotranscriptional recruitment of yeast TRAMP complex to intronic sequences promotes optimal pre-mRNA splicing. Nucleic Acids Res. 42, 643-660.

Kress, T.L., Krogan, N.J., and Guthrie, C. (2008). A Single SR-like Protein, Npl3, Promotes Pre-mRNA Splicing in Budding Yeast. Mol. Cell 32, 727-734.

Kshirsagar, M., and Parker, R. (2004). Identification of Edc3p as an enhancer of mRNA decapping in Saccharomyces cerevisiae. Genetics 166, 729-739.

Kuroha, K., Tatematsu, T., and Inada, T. (2009). Upf1 stimulates degradation of the product derived from aberrant messenger RNA containing a specific nonsense mutation by the proteasome. EMBO Rep. 10, 1265-1271.

Kurosaki, T., Li, W., Hoque, M., Popp, M.W.L., Ermolenko, D.N., Tian, B., and Maquat, L.E. (2014). A Post-Translational regulatory switch on UPF1 controls targeted mRNA degradation. Genes Dev. 28, 1900-1916.

Kurosaki, T., Popp, M.W., and Maquat, L.E. (2019). Quality and quantity control of gene expression by nonsense-mediated mRNA decay. Nat. Rev. Mol. Cell Biol. 
LaGrandeur, T.E., and Parker, R. (1998). Isolation and characterization of Dcp1p, the yeast mRNA decapping enzyme. EMBO J. 17, 1487-1496.

Ledoux, S., and Guthrie, C. (2011). Regulation of the Dbp5 ATPase cycle in mRNP remodeling at the nuclear pore: a lively new paradigm for DEAD-box proteins. Genes Dev. $25,1109-1114$.

Lee-Soety, J.Y., Jones, J., MacGibeny, M.A., Remaly, E.C., Daniels, L., Ito, A., Jean, J., Radecki, H., and Spencer, S. (2012). Yeast hnRNP-related proteins contribute to the maintenance of telomeres. Biochem. Biophys. Res. Commun. 426, 12-17.

Lee, M.S., Henry, M., and Silver, P.A. (1996). A protein that shuttles between the nucleus and the cytoplasm is an important mediator of RNA export. Genes Dev. 10, 1233-1246.

Lee, R.E.C., Brunette, S., Puente, L.G., and Megeney, L.A. (2010). Metacaspase Yca1 is required for clearance of insoluble protein aggregates. Proc. Natl. Acad. Sci. U. S. A. 107, 13348-13353.

Leeds, P., Peltz, S.W., Jacobson, A., and Culbertson, M.R. (1991). The product of the yeast UPF1 gene is required for rapid turnover of mRNAs containing a premature translational termination codon. Genes Dev. 5, 2303-2314.

Leeds, P., Wood, J.M., Lee, B.S., and Culbertson, M.R. (1992). Gene products that promote mRNA turnover in Saccharomyces cerevisiae. Mol. Cell. Biol. 12, 2165-2177.

Lei, E.P. (2001). Messenger RNAs are recruited for nuclear export during transcription. Genes Dev. 15, 1771-1782.

Lejeune, F., and Maquat, L.E. (2005). Mechanistic links between nonsense-mediated mRNA decay and pre-mRNA splicing in mammalian cells. Curr. Opin. Cell Biol. 17, 309315.

Lejeune, F., Ishigaki, Y., Li, X., and Maquat, L.E. (2002). The exon junction complex is detected on CBP80-bound but not elF4E-bound mRNA in mammalian cells: dynamics of mRNP remodeling. EMBO J. 21, 3536-3545.

Lew, J.E., Enomoto, S., and Berman, J. (1998). Telomere length regulation and telomeric chromatin require the nonsense-mediated mRNA decay pathway. Mol. Cell. Biol. 18,61216130. 
Lewis, J.D., Izaurralde, E., Jarmolowski, A., McGuigan, C., and Mattaj, I.W. (1996). A nuclear cap-binding complex facilitates association of U1 snRNP with the cap-proximal 5' splice site. Genes Dev. 10, 1683-1698.

Libri, D., Dower, K., Boulay, J., Thomsen, R., Rosbash, M., and Jensen, T.H. (2002). Interactions between mRNA Export Commitment, 3'-End Quality Control, and Nuclear Degradation. Mol. Cell. Biol. 22, 8254-8266.

Lien, P.T.K., Izumikawa, K., Muroi, K., Irie, K., Suda, Y., and Irie, K. (2016). Analysis of the Physiological Activities of Scd6 through Its Interaction with Hmt1. PLoS One 11, e0164773.

Liu, H., and Naismith, J.H. (2008). An efficient one-step site-directed deletion, insertion, single and multiple-site plasmid mutagenesis protocol. BMC Biotechnol. 8, 91.

Liu, H., Rodgers, N.D., Jiao, X., and Kiledjian, M. (2002). The scavenger mRNA decapping enzyme DcpS is a member of the HIT family of pyrophosphatases. EMBO J. 21, 46994708.

Liu, H.Y., Badarinarayana, V., Audino, D.C., Rappsilber, J., Mann, M., and Denis, C.L. (1998). The NOT proteins are part of the CCR4 transcriptional complex and affect gene expression both positively and negatively. EMBO J. 17, 1096-1106.

Long, J.C., and Caceres, J.F. (2009). The SR protein family of splicing factors: master regulators of gene expression. Biochem. J. 417, 15-27.

Losson, R., and Lacroute, F. (1979). Interference of nonsense mutations with eukaryotic messenger RNA stability. Proc. Natl. Acad. Sci. 76, 5134-5137.

Lykke-Andersen, J., Shu, M.D., and Steitz, J.A. (2001). Communication of the position of exon-exon junctions to the mRNA surveillance machinery by the protein RNPS1. Science 293, 1836-1839.

Maderazo, A.B., He, F., Mangus, D.A., and Jacobson, A. (2000). Upf1p control of nonsense mRNA translation is regulated by Nmd2p and Upf3p. Mol. Cell. Biol. 20, 4591-4603.

Magliery, T.J., Wilson, C.G.M., Pan, W., Mishler, D., Ghosh, I., Hamilton, A.D., and Regan, L. (2005). Detecting protein-protein interactions with a green fluorescent protein fragment reassembly trap: Scope and mechanism. J. Am. Chem. Soc. 127, 146-157.

Mangus, D.A., Evans, M.C., and Jacobson, A. (2003). Poly(A)-binding proteins: multifunctional scaffolds for the post-transcriptional control of gene expression. Genome Biol. 4, 223. 
Mao, X., Schwer, B., and Shuman, S. (1995). Yeast mRNA cap methyltransferase is a 50kilodalton protein encoded by an essential gene. Mol. Cell. Biol. 15, 4167-4174.

Maquat, L.E. (2004). Nonsense-mediated mRNA decay: splicing, translation and mRNP dynamics. Nat. Rev. Mol. Cell Biol. 5, 89-99.

Maquat, L.E., Hwang, J., Sato, H., and Tang, Y. (2010). CBP80-promoted mRNP rearrangements during the pioneer round of translation, nonsense-mediated mRNA decay, and thereafter. Cold Spring Harb. Symp. Quant. Biol. 75, 127-134.

Marquardt, S., Hazelbaker, D.Z., and Buratowski, S. (2011). Distinct RNA degradation pathways and 3' extensions of yeast non-coding RNA species. Transcription 2, 145-154.

Martínez-Lumbreras, S., Taverniti, V., Zorrilla, S., Séraphin, B., and Pérez-Cañadillas, J.M. (2016). Gbp2 interacts with THO/TREX through a novel type of RRM domain. Nucleic Acids Res. 44, 437-448.

Mayeda, A., Badolato, J., Kobayashi, R., Zhang, M.Q., Gardiner, E.M., and Krainer, A.R. (1999). Purification and characterization of human RNPS1: a general activator of pre-mRNA splicing. EMBO J. 18, 4560-4570.

McGlincy, N.J., and Smith, C.W.J. (2008). Alternative splicing resulting in nonsensemediated mRNA decay: what is the meaning of nonsense? Trends Biochem. Sci. 33, 385393.

Meaux, S., van Hoof, A., and Baker, K.E. (2008). Nonsense-mediated mRNA decay in yeast does not require PAB1 or a poly $(A)$ tail. Mol. Cell 29, 134-140.

Medghalchi, S.M., Frischmeyer, P.A., Mendell, J.T., Kelly, A.G., Lawler, A.M., and Dietz, H.C. (2001). Rent1, a trans-effector of nonsense-mediated mRNA decay, is essential for mammalian embryonic viability. Hum. Mol. Genet. 10, 99-105.

Meinel, D.M., and Sträßer, K. (2015). Co-transcriptional mRNP formation is coordinated within a molecular mRNP packaging station in S. cerevisiae. Bioessays.

Mendell, J.T., Medghalchi, S.M., Lake, R.G., Noensie, E.N., and Dietz, H.C. (2000). Novel Upf2p orthologues suggest a functional link between translation initiation and nonsense surveillance complexes. Mol. Cell. Biol. 20, 8944-8957.

Metzstein, M.M., and Krasnow, M.A. (2006). Functions of the Nonsense-Mediated mRNA Decay Pathway in Drosophila Development. PLoS Genet. 2, e180. 
Min, E.E., Roy, B., Amrani, N., He, F., and Jacobson, A. (2013). Yeast Upf1 CH domain interacts with Rps26 of the 40S ribosomal subunit. RNA 19, 1105-1115.

Mitchell, P., and Tollervey, D. (2003). An NMD Pathway in Yeast Short Article Involving Accelerated Deadenylation and Exosome-Mediated $3 \stackrel{-}{\rightarrow} 5$ Degradation. $11,1405-1413$.

Moehle, E.A., Ryan, C.J., Krogan, N.J., Kress, T.L., and Guthrie, C. (2012). The Yeast SRLike Protein Npl3 Links Chromatin Modification to mRNA Processing. PLoS Genet. 8, e1003101.

Muhlrad, D., and Parker, R. (1994). Premature translational termination triggers mRNA decapping. Nature 370, 578-581.

Muhlrad, D., and Parker, R. (1999a). Aberrant mRNAs with extended 3' UTRs are substrates for rapid degradation by mRNA surveillance. RNA 5, 1299-1307.

Muhlrad, D., and Parker, R. (1999b). Recognition of Yeast mRNAs as "Nonsense Containing" Leads to Both Inhibition of mRNA Translation and mRNA Degradation: Implications for the Control of mRNA Decapping. Mol. Biol. Cell 10, 3971-3978.

Muhlrad, D., and Parker, R. (2005). The yeast EDC1 mRNA undergoes deadenylationindependent decapping stimulated by Not2p, Not4p, and Not5p. EMBO J. 24, 1033-1045.

Muhlrad, D., Decker, C.J., and Parker, R. (1994). Deadenylation of the unstable mRNA encoded by the yeast MFA2 gene leads to decapping followed by 5'--\&gt;3' digestion of the transcript. Genes Dev. 8, 855-866.

Nott, A., Le Hir, H., and Moore, M.J. (2004). Splicing enhances translation in mammalian cells: an additional function of the exon junction complex. Genes Dev. 18, 210-222.

Okada-Katsuhata, Y., Yamashita, A., Kutsuzawa, K., Izumi, N., Hirahara, F., and Ohno, S. (2012). N- and C-terminal Upf1 phosphorylations create binding platforms for SMG-6 and SMG-5:SMG-7 during NMD. Nucleic Acids Res. 40, 1251-1266.

Park, E.-H., Walker, S.E., Lee, J.M., Rothenburg, S., Lorsch, J.R., and Hinnebusch, A.G. (2011). Multiple elements in the elF4G1 N-terminus promote assembly of elF4G1 PABP mRNPs in vivo. EMBO J. 30, 302-316.

Park, Y.-U., Hur, H., Ka, M., and Kim, J. (2006). Identification of translational regulation target genes during filamentous growth in Saccharomyces cerevisiae: regulatory role of Caf20 and Dhh1. Eukaryot. Cell 5, 2120-2127. 
Peltz, S.W., Donahue, J.L., and Jacobson, A. (1992). A mutation in the tRNA nucleotidyltransferase gene promotes stabilization of mRNAs in Saccharomyces cerevisiae. Mol. Cell. Biol. 12, 5778-5784.

Peltz, S.W., Brown, A.H., and Jacobson, A. (1993). mRNA destabilization triggered by premature translational termination depends on at least three cis-acting sequence elements and one trans-acting factor. Genes Dev. 7, 1737-1754.

Peña, A., Gewartowski, K., Mroczek, S., Cuéllar, J., Szykowska, A., Prokop, A., CzarnockiCieciura, M., Piwowarski, J., Tous, C., Aguilera, A., et al. (2012). Architecture and nucleic acids recognition mechanism of the THO complex, an mRNP assembly factor. EMBO J. 31, 1605-1616.

Pisarev, A. V, Skabkin, M.A., Pisareva, V.P., Skabkina, O. V, Rakotondrafara, A.M., Hentze, M.W., Hellen, C.U.T., and Pestova, T. V (2010). The role of ABCE1 in eukaryotic posttermination ribosomal recycling. Mol. Cell 37, 196-210.

Preis, A., Heuer, A., Barrio-Garcia, C., Hauser, A., Eyler, D.E., Berninghausen, O., Green, R., Becker, T., and Beckmann, R. (2014). Cryoelectron Microscopic Structures of Eukaryotic Translation Termination Complexes Containing eRF1-eRF3 or eRF1-ABCE1. Cell Rep. 8, 59-65.

Ptushkina, M., von der Haar, T., Vasilescu, S., Frank, R., Birkenhäger, R., and McCarthy, J.E. (1998). Cooperative modulation by elF4G of elF4E-binding to the mRNA 5' cap in yeast involves a site partially shared by p20. EMBO J. 17, 4798-4808.

Qiu, Z.R., Chico, L., Chang, J., Shuman, S., and Schwer, B. (2012). Genetic interactions of hypomorphic mutations in the $\mathrm{m} 7 \mathrm{G}$ cap-binding pocket of yeast nuclear cap binding complex: an essential role for Cbc2 in meiosis via splicing of MER3 pre-mRNA. RNA 18, 1996-2011.

Rajyaguru, P., She, M., and Parker, R. (2012). Scd6 targets elF4G to repress translation: RGG motif proteins as a class of elF4G-binding proteins. Mol. Cell 45, 244-254.

Ramirez, C.V., Vilela, C., Berthelot, K., and McCarthy, J.E.G. (2002). Modulation of eukaryotic mRNA stability via the cap-binding translation complex elF4F. J. Mol. Biol. 318, 951-962. 
Richardson, R., Denis, C.L., Zhang, C., Nielsen, M.E.O., Chiang, Y.-C., Kierkegaard, M., Wang, X., Lee, D.J., Andersen, J.S., and Yao, G. (2012). Mass spectrometric identification of proteins that interact through specific domains of the poly $(A)$ binding protein. Mol. Genet. Genomics 287, 711-730.

Roque, S., Cerciat, M., Gaugué, I., Mora, L., Floch, A.G., de Zamaroczy, M., HeurguéHamard, V., and Kervestin, S. (2015). Interaction between the poly(A)-binding protein Pab1 and the eukaryotic release factor eRF3 regulates translation termination but not mRNA decay in Saccharomyces cerevisiae. RNA 21, 124-134.

Rose, M.D.., Winston, F.., and Hieter, P. (1990). Methods in Yeast Genetics, A Laboratory Course Manual. Cold Spring Harbor Laboratory. In Biochemistry and Molecular Biology Education, (NY: Cold Spring Harbor), pp. 101-102.

Ruiz-Echevarria, M.J., and Peltz, S.W. (1996). Utilizing the GCN4 leader region to investigate the role of the sequence determinants in nonsense-mediated mRNA decay. EMBO J. 15, 2810-2819.

Ruiz-Echevarría, M.J., and Peltz, S.W. (2000). The RNA binding protein Pub1 modulates the stability of transcripts containing upstream open reading frames. Cell 101, 741-751.

Ruiz-Echevarría, M.J., González, C.I., and Peltz, S.W. (1998). Identifying the right stop: determining how the surveillance complex recognizes and degrades an aberrant mRNA. EMBO J. 17, 575-589.

Russell, I.D., and Tollervey, D. (1992). NOP3 is an essential yeast protein which is required for pre-rRNA processing. J. Cell Biol. 119, 737-747.

Sambrook, J., Fritsch, E.F., and Maniatis, T. (1989). Molecular cloning: a laboratory manual. Mol. Cloning a Lab. Manual.

San Paolo, S., Vanacova, S., Schenk, L., Scherrer, T., Blank, D., Keller, W., and Gerber, A.P. (2009). Distinct roles of non-canonical poly(A) polymerases in RNA metabolism. PLoS Genet. 5, e1000555.

Sanford, J.R. (2004). A novel role for shuttling SR proteins in mRNA translation. Genes Dev. 18, 755-768.

Santos-Rosa, H., Moreno, H., Simos, G., Segref, A., Fahrenkrog, B., Pante, N., and Hurt, E. (1998). Nuclear mRNA Export Requires Complex Formation between Mex67p and Mtr2p at the Nuclear Pores. Mol. Cell. Biol. 18, 6826-6838. 
Sato, H., Hosoda, N., and Maquat, L.E. (2008). Efficiency of the Pioneer Round of Translation Affects the Cellular Site of Nonsense-Mediated mRNA Decay. Mol. Cell 29, 255-262.

Schwartz, D.C., and Parker, R. (2000). mRNA decapping in yeast requires dissociation of the cap binding protein, eukaryotic translation initiation factor 4E. Mol. Cell. Biol. 20, 79337942.

Schwartz, D., Decker, C.J., and Parker, R. (2003). The enhancer of decapping proteins, Edc1p and Edc2p, bind RNA and stimulate the activity of the decapping enzyme. RNA 9, 239-251.

Segal, S.P., Dunckley, T., and Parker, R. (2006). Sbp1p Affects Translational Repression and Decapping in Saccharomyces cerevisiae. Mol. Cell. Biol. 26, 5120-5130.

Segref, A., Sharma, K., Doye, V., Hellwig, A., Huber, J., Lührmann, R., and Hurt, E. (1997). Mex67p, a novel factor for nuclear mRNA export, binds to both poly $(A)+$ RNA and nuclear pores. EMBO J. 16, 3256-3271.

Sei, E., and Conrad, N.K. (2014). UV Cross-Linking of Interacting RNA and Protein in Cultured Cells. In Methods in Enzymology, pp. 53-66.

Sellick, C.A., Campbell, R.N., and Reece, R.J. (2008). Chapter 3 Galactose Metabolism in Yeast-Structure and Regulation of the Leloir Pathway Enzymes and the Genes Encoding Them. Int. Rev. Cell Mol. Biol.

Serdar, L.D., Whiteside, D.L., and Baker, K.E. (2016). ATP hydrolysis by UPF1 is required for efficient translation termination at premature stop codons. Nat. Commun. 7, 14021.

Sherman, F. (2002). Getting started with yeast. Methods Enzymol. 350, 3-41.

Sherman, F., and Hicks, J. (1991). [2] Micromanipulation and dissection of asci. Methods Enzymol. 194, 21-37.

Sheth, U., and Parker, R. (2003). Decapping and decay of messenger RNA occur in cytoplasmic processing bodies. Science 300, 805-808.

Sheth, U., and Parker, R. (2006). Targeting of aberrant mRNAs to cytoplasmic processing bodies. Cell 125, 1095-1109. 
Shirley, R.L., Lelivelt, M.J., Schenkman, L.R., Dahlseid, J.N., and Culbertson, M.R. (1998). A factor required for nonsense-mediated mRNA decay in yeast is exported from the nucleus to the cytoplasm by a nuclear export signal sequence. J. Cell Sci. 111 ( Pt 21), 3129-3143.

Shoemaker, C.J., and Green, R. (2011). Kinetic analysis reveals the ordered coupling of translation termination and ribosome recycling in yeast. Proc. Natl. Acad. Sci. U. S. A. 108, E1392-8.

Shoemaker, C.J., Eyler, D.E., and Green, R. (2010). Dom34:Hbs1 promotes subunit dissociation and peptidyl-tRNA drop-off to initiate no-go decay. Science 330, 369-372.

Siebel, C.W., and Guthrie, C. (1996). The essential yeast RNA binding protein Npl3p is methylated. Proc. Natl. Acad. Sci. 93, 13641-13646.

Sikorski, R.S., and Hieter, P. (1989). A system of shuttle vectors and yeast host strains designed for efficient manipulation of DNA in Saccharomyces cerevisiae. Genetics 122, 19 27.

Singh, G., Kucukural, A., Cenik, C., Leszyk, J.D., Shaffer, S.A., Weng, Z., and Moore, M.J. (2012). The Cellular EJC Interactome Reveals Higher-Order mRNP Structure and an EJCSR Protein Nexus. Cell 151, 915-916.

Sinturel, F., Bréchemier-Baey, D., Kiledjian, M., Condon, C., and Bénard, L. (2012). Activation of $5^{\prime}-3^{\prime}$ exoribonuclease Xrn1 by cofactor Dcs1 is essential for mitochondrial function in yeast. Proc. Natl. Acad. Sci. U. S. A. 109, 8264-8269.

Sloan, K.E., Schneider, C., and Watkins, N.J. (2012). Comparison of the yeast and human nuclear exosome complexes. Biochem. Soc. Trans. 40, 850-855.

Solinger, J.A., Pascolini, D., and Heyer, W.D. (1999). Active-site mutations in the Xrn1p exoribonuclease of Saccharomyces cerevisiae reveal a specific role in meiosis. Mol. Cell. Biol. 19, 5930-5942.

Sprague, G.F. (1991). [5] Assay of yeast mating reaction. Methods Enzymol. 194, 77-93.

Steiger, M., Carr-Schmid, A., Schwartz, D.C., Kiledjian, M., and Parker, R. (2003). Analysis of recombinant yeast decapping enzyme. RNA 9, 231-238.

Stewart, M. (2010). Nuclear export of mRNA. Trends Biochem. Sci. 35, 609-617.

Strambio-De-Castillia, C., Niepel, M., and Rout, M.P. (2010). The nuclear pore complex: bridging nuclear transport and gene regulation. Nat. Rev. Mol. Cell Biol. 11, 490-501. 
Strasser, K., Bassler, J., and Hurt, E. (2000). Binding of the Mex67p/Mtr2p Heterodimer to Fxfg, Glfg, and Fg Repeat Nucleoporins Is Essential for Nuclear mRNA Export. J. Cell Biol. 150, 695-706.

Stutz, F., Bachi, A., Doerks, T., Braun, I.C., Seraphin, B., Wilm, M., Bork, P., and Izaurralde, E. (2000). REF, an evolutionary conserved family of hnRNP-like proteins, interacts with TAP/Mex67p and participates in mRNA nuclear export. RNA 6, 638-650.

Swisher, K.D., and Parker, R. (2011). Interactions between Upf1 and the decapping factors Edc3 and Pat1 in Saccharomyces cerevisiae. PLoS One 6, e26547.

Synowsky, S.A., and Heck, A.J.R. (2008). The yeast Ski complex is a hetero-tetramer. Protein Sci. 17, 119-125.

Takahashi, S., Araki, Y., Sakuno, T., and Katada, T. (2003). Interaction between Ski7p and Upf $1 p$ is required for nonsense-mediated 3'-to-5' mRNA decay in yeast. EMBO J. 22, 39513959.

Takahashi, S., Araki, Y., Ohya, Y., Sakuno, T., Hoshino, S.-I., Kontani, K., Nishina, H., and Katada, T. (2008). Upf1 potentially serves as a RING-related E3 ubiquitin ligase via its association with Upf3 in yeast. RNA 14, 1950-1958.

Taura, T., Krebber, H., and Silver, P.A. (1998). A member of the Ran-binding protein family, Yrb2p, is involved in nuclear protein export. Proc. Natl. Acad. Sci. U. S. A. 95, 7427-7432.

Tharun, S., and Parker, R. (2001). Targeting an mRNA for decapping: displacement of translation factors and association of the Lsm1p-7p complex on deadenylated yeast mRNAs. Mol. Cell 8, 1075-1083.

Tharun, S., He, W., Mayes, A.E., Lennertz, P., Beggs, J.D., and Parker, R. (2000). Yeast Sm-like proteins function in mRNA decapping and decay. Nature 404, 515-518.

Thompson, D.M., and Parker, R. (2007). Cytoplasmic decay of intergenic transcripts in Saccharomyces cerevisiae. Mol. Cell. Biol. 27, 92-101.

Tieg, B., and Krebber, H. (2013). Dbp5 - from nuclear export to translation. Biochim. Biophys. Acta 1829, 791-798.

Toma, K.G., Rebbapragada, I., Durand, S., and Lykke-Andersen, J. (2015). Identification of elements in human long 3' UTRs that inhibit nonsense-mediated decay. RNA 21, 887-897. 
Tsuboi, T., Kuroha, K., Kudo, K., Makino, S., Inoue, E., Kashima, I., and Inada, T. (2012). Dom34:hbs1 plays a general role in quality-control systems by dissociation of a stalled ribosome at the 3' end of aberrant mRNA. Mol. Cell 46, 518-529.

Tsukamoto, T., Shibagaki, Y., Imajoh-Ohmi, S., Murakoshi, T., Suzuki, M., Nakamura, A., Gotoh, H., and Mizumoto, K. (1997). Isolation and Characterization of the Yeast mRNA Capping Enzyme $\beta$ Subunit Gene Encoding RNA 5'-Triphosphatase, Which Is Essential for Cell Viability. Biochem. Biophys. Res. Commun. 239, 116-122.

Tuck, A.C., and Tollervey, D. (2013). A transcriptome-wide atlas of RNP composition reveals diverse classes of mRNAs and IncRNAs. Cell 154, 996-1009.

Tucker, M., Valencia-Sanchez, M.A., Staples, R.R., Chen, J., Denis, C.L., and Parker, R. (2001). The transcription factor associated Ccr4 and Caf1 proteins are components of the major cytoplasmic mRNA deadenylase in Saccharomyces cerevisiae. Cell 104, 377-386.

Tucker, M., Staples, R.R., Valencia-Sanchez, M.A., Muhlrad, D., and Parker, R. (2002). Ccr4p is the catalytic subunit of a Ccr4p/Pop2p/Notp mRNA deadenylase complex in Saccharomyces cerevisiae. EMBO J. 21, 1427-1436.

Tutucci, E., and Stutz, F. (2011). Keeping mRNPs in check during assembly and nuclear export. Nat. Rev. Mol. Cell Biol. 12, 377-384.

Unterholzner, L., and Izaurralde, E. (2004). SMG7 acts as a molecular link between mRNA surveillance and mRNA decay. Mol. Cell 16, 587-596.

Urakov, V.N., Valouev, I.A., Lewitin, E.I., Paushkin, S. V, Kosorukov, V.S., Kushnirov, V. V, Smirnov, V.N., and Ter-Avanesyan, M.D. (2001). Itt1p, a novel protein inhibiting translation termination in Saccharomyces cerevisiae. BMC Mol. Biol. 2, 9.

Urakov, V.N., Mitkevich, O. V, Safenkova, I. V, and Ter-Avanesyan, M.D. (2017). Ribosome-bound Pub1 modulates stop codon decoding during translation termination in yeast. FEBS J. 284, 1914-1930.

Vilela, C., Velasco, C., Ptushkina, M., and McCarthy, J.E. (2000). The eukaryotic mRNA decapping protein Dcp1 interacts physically and functionally with the elF4F translation initiation complex. EMBO J. 19, 4372-4382.

Wang, J., Vock, V.M., Li, S., Olivas, O.R., and Wilkinson, M.F. (2002a). A quality control pathway that down-regulates aberrant T-cell receptor (TCR) transcripts by a mechanism requiring UPF2 and translation. J. Biol. Chem. 277, 18489-18493. 
Wang, J., Gudikote, J.P., Olivas, O.R., and Wilkinson, M.F. (2002b). Boundary-independent polar nonsense-mediated decay. EMBO Rep. 3, 274-279.

Wang, W., Czaplinski, K., Rao, Y., and Peltz, S.W. (2001). The role of Upf proteins in modulating the translation read-through of nonsense-containing transcripts. EMBO J. 20 , 880-890.

Wang, W., Cajigas, I.J., Peltz, S.W., Wilkinson, M.F., and Gonzalez, C.I. (2006). Role for Upf2p Phosphorylation in Saccharomyces cerevisiae Nonsense-Mediated mRNA Decay. Mol. Cell. Biol. 26, 3390-3400.

Welch, E.M., and Jacobson, A. (1999). An internal open reading frame triggers nonsensemediated decay of the yeast SPT10 mRNA. EMBO J. 18, 6134-6145.

Wells, S.E., Hillner, P.E., Vale, R.D., and Sachs, A.B. (1998). Circularization of mRNA by eukaryotic translation initiation factors. Mol. Cell 2, 135-140.

Wery, M., Descrimes, M., Vogt, N., Dallongeville, A.-S., Gautheret, D., and Morillon, A. (2016). Nonsense-Mediated Decay Restricts LncRNA Levels in Yeast Unless Blocked by Double-Stranded RNA Structure. Mol. Cell 61, 379-392.

Wiegand, H.L., Lu, S., and Cullen, B.R. (2003). Exon junction complexes mediate the enhancing effect of splicing on mRNA expression. Proc. Natl. Acad. Sci. U. S. A. 100, 11327-11332.

Wilkie, G.S., Dickson, K.S., and Gray, N.K. (2003). Regulation of mRNA translation by 5'and 3'-UTR-binding factors. Trends Biochem. Sci. 28, 182-188.

Will, C.L., and Lührmann, R. (2011). Spliceosome structure and function. Cold Spring Harb. Perspect. Biol. 3, 1-2.

Wilson, G.M., and Brewer, G. (1999). The search for trans-acting factors controlling messenger RNA decay. Prog. Nucleic Acid Res. Mol. Biol. 62, 257-291.

Wilusz, C.J., Gao, M., Jones, C.L., Wilusz, J., and Peltz, S.W. (2001). Poly(A)-binding proteins regulate both $\mathrm{mRNA}$ deadenylation and decapping in yeast cytoplasmic extracts. RNA 7, 1416-1424.

Windgassen, M., and Krebber, H. (2003). Identification of Gbp2 as a novel poly(A)+ RNAbinding protein involved in the cytoplasmic delivery of messenger RNAs in yeast. EMBO Rep. 4, 278-283. 
Windgassen, M., Sturm, D., Cajigas, I.J., Gonzalez, C.I., Seedorf, M., Bastians, H., and Krebber, H. (2004). Yeast Shuttling SR Proteins Npl3p, Gbp2p, and Hrb1p Are Part of the Translating mRNPs, and Npl3p Can Function as a Translational Repressor. Mol. Cell. Biol. 24, 10479-10491.

Wong, C.-M., Qiu, H., Hu, C., Dong, J., and Hinnebusch, A.G. (2007). Yeast cap binding complex impedes recruitment of cleavage factor IA to weak termination sites. Mol. Cell. Biol. $27,6520-6531$.

Wong, C.-M., Tang, H.-M.V., Kong, K.-Y.E., Wong, G.-W.O., Qiu, H., Jin, D.-Y., and Hinnebusch, A.G. (2010). Yeast arginine methyltransferase Hmt1p regulates transcription elongation and termination by methylating Npl3p. Nucleic Acids Res. 38, 2217-2228.

Xu, C., Henry, P.A., Setya, A., and Henry, M.F. (2003). In vivo analysis of nucleolar proteins modified by the yeast arginine methyltransferase Hmt1/Rmt1p. RNA 9, 746-759.

Yamashita, A., Chang, T.-C., Yamashita, Y., Zhu, W., Zhong, Z., Chen, C.-Y.A., and Shyu, A.-B. (2005). Concerted action of poly (A) nucleases and decapping enzyme in mammalian mRNA turnover. Nat. Struct. Mol. Biol. 12, 1054-1063.

Yamashita, A., Izumi, N., Kashima, I., Ohnishi, T., Saari, B., Katsuhata, Y., Muramatsu, R., Morita, T., Iwamatsu, A., Hachiya, T., et al. (2009). SMG-8 and SMG-9, two novel subunits of the SMG-1 complex, regulate remodeling of the mRNA surveillance complex during nonsense-mediated mRNA decay. Genes Dev. 23, 1091-1105.

Yoine, M., Nishii, T., and Nakamura, K. (2006). Arabidopsis UPF1 RNA helicase for nonsense-mediated mRNA decay is involved in seed size control and is essential for growth. Plant Cell Physiol. 47, 572-580.

Yun, D.F., and Sherman, F. (1995). Initiation of translation can occur only in a restricted region of the CYC1 mRNA of Saccharomyces cerevisiae. Mol. Cell. Biol. 15, 1021-1033.

Zander, G., Hackmann, A., Bender, L., Becker, D., Lingner, T., Salinas, G., and Krebber, $H$. (2016). mRNA quality control is bypassed for immediate export of stress-responsive transcripts. Nature 540, 593-596.

Zhang, Z., and Krainer, A.R. (2004). Involvement of SR proteins in mRNA surveillance. Mol. Cell 16, 597-607. 
Zhang, S., Ruiz-Echevarria, M.J., Quan, Y., and Peltz, S.W. (1995). Identification and characterization of a sequence motif involved in nonsense-mediated mRNA decay. Mol. Cell. Biol. 15, 2231-2244.

Zhang, S., Welch, E.M., Hogan, K., Brown, A.H., Peltz, S.W., and Jacobson, A. (1997). Polysome-associated mRNAs are substrates for the nonsense-mediated mRNA decay pathway in Saccharomyces cerevisiae. RNA 3, 234-244.

Zhao, J., Hyman, L., and Moore, C. (1999). Formation of mRNA 3' Ends in Eukaryotes: Mechanism, Regulation, and Interrelationships with Other Steps in mRNA Synthesis. Microbiol. Mol. Biol. Rev. 63, 405-445.

Zuk, D., and Jacobson, A. (1998). A single amino acid substitution in yeast elF-5A results in mRNA stabilization. EMBO J. 17, 2914-2925. 


\section{Acknowledgement}

I would like to express my appreciation to everyone who supported me during my work. In particular, I wish to extend my special gratitude to Prof. Dr. Heike Krebber for giving me the opportunity to work in this interesting field as well as her great supervision over the course of four years.

The assistance and the advice provided by my Thesis Committee members Prof. Dr. Reinhard Lührmann and Prof. Dr. Jörg Großhans proved valuable and were greatly appreciated.

Further, I wish to acknowledge the department of Prof. Dr. Gerhard Braus, for providing several yeast strains, and everyone mentioned in the Materials and Methods sections who provided plasmids, strains or antibodies.

I would like to thank the former and current members in the departments of Prof. Dr. Heike Krebber and Prof. Dr. Holger Bastians for the great teamwork and valuable advice. A special thanks goes to Dr. Alexandra Hackmann, who supervised me in the early phase of my work and helped me greatly to start this project. Further, I am particularly grateful that Yen-Yun Lu joined me in this project - not only for her great contribution but also for the invaluable scientific discussions.

Finally, I wish to thank Sari Siska for all her support, motivation and understanding. 